Florida International University FIU Digital Commons

9-14-2012

\title{
Angular Distribution of Prompt Photons Using the Compact Muon Solenoid Detector at $\sqrt{S}=7 \mathrm{TeV}$
}

Vanessa Gaultney Werner

Florida International University, vgaul001@fiu.edu

DOI: $10.25148 /$ etd.FI12111303

Follow this and additional works at: https://digitalcommons.fiu.edu/etd

\section{Recommended Citation}

Werner, Vanessa Gaultney, "Angular Distribution of Prompt Photons Using the Compact Muon Solenoid Detector at $\sqrt{ } \mathrm{S}=7 \mathrm{TeV}$ " (2012). FIU Electronic Theses and Dissertations. 727.

https://digitalcommons.fiu.edu/etd/727 


\title{
FLORIDA INTERNATIONAL UNIVERSITY \\ Miami, Florida
}

\section{ANGULAR DISTRIBUTION OF PROMPT PHOTONS USING THE COMPACT MUON SOLENOID DETECTOR AT $\sqrt{S}=7 \mathrm{TEV}$}

A dissertation submitted in partial fulfillment of the requirements for the degree of DOCTOR OF PHILOSOPHY

\author{
in \\ PHYSICS \\ by \\ Vanessa Gaultney Werner
}

2012 


\section{To: Dean Kenneth Furton}

College of Arts and Sciences

This dissertation, written by Vanessa Gaultney Werner, and entitled Angular Distribution of Prompt Photons Using The Compact Muon Solenoid Detector at $\sqrt{S}=7$ $\mathrm{TeV}$, having been approved in respect to style and intellectual content, is referred to you for judgment.

We have read this dissertation and recommend that it be approved.

Stephan Linn

Jorge Rodriguez

Joerg Reinhold

Eric Brewe

Pete Markowitz, Major Professor

Date of Defense: September 14, 2012

The dissertation of Vanessa Gaultney Werner is approved.

Dean Kenneth Furton
College of Arts and Sciences

Dean Lakshmi N. Reddi
University Graduate School

Florida International University, 2012 


\section{DEDICATION}

This work is dedicated to my mother, Nora Gaultney. 


\section{ACKNOWLEDGMENTS}

I must first and foremost acknowledge and thank the other physics warriors from FIU with whom I began this long journey with; Cristobal Ceron, Diane Alvarez, Betty Brenner, Priscilla Pamela, Brian Beckford, Alejandro De La Puente, and Robert Alvarez. From studying for hours at the library to the VH conference room, we were all there for each other and I will never forget that. I will forever cherish the time we spent together, even if our discussions mostly dealt with relativistic kinematics. I'd like to give a special thanks to Luis Lebolo, the other particle physics student at FIU, who was always there to lend a hand when I needed it...'Hey Luis!'...

I thank my husband, Jeremy Scott Werner, who continually pushed me to do my best and believed in me every step of the way.

I thank my mother, Nora Gaultney, for whom this work is dedicated to. I also thank the rest of my immediate family members; my father Orlando Gomez, my brother Diego Gomez, my uncles Carlos 'Cali' Nino, Luis 'Nando' Nino, and Jose 'Cobe' Nino, my aunt Angela Zuluaga, my cousin Johanna Santacoloma and her children Luis, Javier and Tatyanna Acevedo, and my sister Marcia Gomez and her daughter Aaliyah Velazquez. I thank my grandparents Leonora and Luis Nino for migrating to the USA from Colombia in order to give our family a better life, I hope they are proud of everything we've accomplished in our lives. Te extraño Mama y Papucho.

I'd like to thank the fellow students, researchers and professors I met along the way that gave me insight into the world of physics and who helped shape (in no particular order); German Martinez, Andrew Askew, Yuri Gerstein, Vashundara Chetluru, Vivian O'Dell, Shruti Shrestha, Michael Anderson, Richard Cavanaugh, Ian Shipsey, Vasken Hagopian, Andrew Kubik, Steven Won, Adriaan Heering, Tina Vernon, Richard Kellogg, Toyoko Onimoto, and Susanne Gascon-Shotkin. I'd like to 
especially thank Shin-Shan 'Eiko' Yu, whose invaluable knowledge about QCD, event generators and physics has helped greatly during this process.

A special thanks goes to Dr. Laird Kramer who introduced me to the world of physics and all that it had to offer. I would have never had the opportunity to teach, be a mentor, travel across the world and learn about the smallest known particles had it not been for you.

I thank my committee members Dr. Joerg Reinhold and Dr. Eric Brewe for taking the time to evaluate my progress from the beginning of my graduate studies to my thesis proposal and following up to my doctoral defense.

A special thanks to the remaining committee members and professors of the particles physics group at FIU; Dr. Pete Markowitz, Dr. Jorge Rodgriguez and Dr. Stephan Linn. Without your strong, much needed guidance you all gave me through the minefield that is particle physics field I would not have come out alive on the other side. I have come to think of you all as part of my family throughout the years. There are not many groups like the one at FIU where the student-mentor relationship is real and I am very fortunate to of had such an experience.

I also like to thank all the other scientists, engineers and theorists who I do not know personally, but are fellow collaborators and contributors to the Compact Muon Solenoid experiment and the Large Hadron Collider. They had the commitment, innovation and ambition to design an experiment like no other, stick with it for decades and see it all the way through. They laid the foundation for future students to expand their knowledge for years to come. 


\author{
ABSTRACT OF THE DISSERTATION \\ ANGULAR DISTRIBUTION OF PROMPT PHOTONS USING THE COMPACT \\ MUON SOLENOID DETECTOR AT $\sqrt{S}=7 \mathrm{TEV}$ \\ by \\ Vanessa Gaultney Werner \\ Florida International University, 2012 \\ Miami, Florida \\ Professor Pete Markowitz, Major Professor \\ Professor Stephan Linn, Major Co-Professor
}

The study of the angular distribution of photon plus jet events in $p p$ collisions at $\sqrt{S}=7 \mathrm{TeV}$ with the Compact Muon Solenoid (CMS) detector is presented. The photon is restricted to the central region of the detector $(|\eta|<1.4442)$ while the jet is allowed to be present in both central and forward regions of CMS $(|\eta|<2.4)$. Dominant backgrounds due to jets fragmenting into neutral mesons are accounted for through the use of a template method that discriminates between signal and background. The angular distribution, $\left|\eta^{*}\right|$, is defined as the absolute value of the difference in $\eta$ between the leading photon and leading jet in an event divided by two. The angular distribution ranging from 0-1.4 was examined and compared with next-to-leading order QCD predictions and was found to be in good agreement. 
1 THEORY 1

1.1 The Standard Model . . . . . . . . . . . . . . . . . . . . . . . . . 2

1.2 The Parton Model and QCD $\ldots \ldots \ldots \ldots \ldots$

1.2 .1 Leading Order Calculation . . . . . . . . . . . . . . . . . 14

1.2 .2 Next to Leading Order Calculation . . . . . . . . . . . . 15

2 THE COMPACT MUON SOLENOID DETECTOR 22

2.1 The Large Hadron Collider . . . . . . . . . . . . . . . . . . . . . . . 22

2.2 The Compact Muon Solenoid Detector _ . . . . . . . . . . . . . . 25

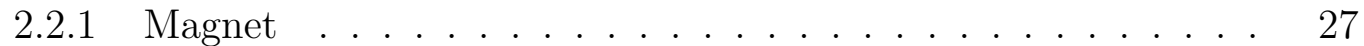

2.2 .2 The Tracking System . . . . . . . . . . . . . . . . . . . . 28

2.2 .3 The Calorimeters at CMS $\ldots \ldots \ldots \ldots \ldots$

2.2 .4 The Trigger System . . . . . . . . . . . . . . . . . . . . . . . . . 38

2.3 The CMS Computing Infrastructure $\ldots \ldots \ldots \ldots$

3 OFFLINE COMPUTING 45

3.1 Photon Reconstruction . . . . . . . . . . . . . . . . . . . . 45

3.2 Jet Reconstruction . . . . . . . . . . . . . . . . . . . . . . . . 47

3.2 .1 The Particle Flow Algorithm. . . . . . . . . . . . . 47

3.2 .2 The Anti-k $\mathrm{k}_{T}$ Algorithm $\ldots \ldots \ldots \ldots$. . . . . . . . . . . . . 49

$3.2 .3 \quad$ Jet Energy Scale $\ldots \ldots \ldots \ldots$

4 EVENT SELECTION

4.1 Monte Carlo Samples . . . . . . . . . . . . . . . . . . . . . . . . . . . 52

4.1 .1 Pile-up Effects.$\ldots \ldots \ldots$

4.2 Data Samples $\ldots \ldots \ldots \ldots \ldots$

4.3 Variables Used for Analysis $\ldots \ldots \ldots \ldots \ldots$

$4.4 \quad$ Event Quality Cuts $\ldots \ldots \ldots \ldots \ldots$

$4.5 \quad$ Efficiencies $\ldots \ldots \ldots \ldots \ldots \ldots$

5 BACKGROUND ESTIMATION 67

5.1 Signal and Background Discrimination . . . . . . . . . . . 67

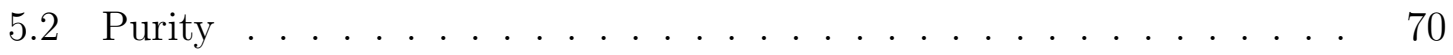

6 SYSTEMATIC UNCERTAINTIES 76

6.1 Background Shape . . . . . . . . . . . . . . . . . . . . 76

6.2 Jet $\eta$ Position Resolution . . . . . . . . . . . . . . . . . . . 78

7 THE ANGULAR DISTRIBUTION 82

7.1 NLO Theoretical Prediction . . . . . . . . . . . . . . . . . . . . . 82

7.1 .1 Theoretical Uncertainty $\ldots \ldots \ldots \ldots$ 
7.2 Results . . . . . . . . . . . . . . . . . . . . . . . . . . . . . . . 84

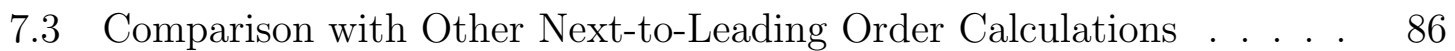

7.4 Conclusions $\ldots \ldots \ldots \ldots \ldots \ldots \ldots \ldots \ldots \ldots$

$\begin{array}{ll}\text { REFERENCES } & 92\end{array}$

\begin{tabular}{ll}
\hline APPENDICES & 97
\end{tabular}

VITA 151 
1.1 Table of partons and their properties [1]. . . . . . . . . . . . . . . . 4

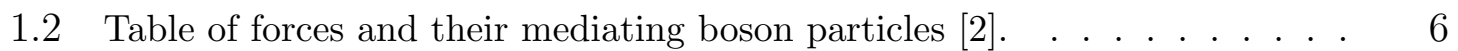

1.3 Partonic differential cross sections for prompt photon production at leading order [3]. The dependencies on $\pi, \alpha_{s}, \alpha$ and $\hat{s}$ have been removed for simplicity. 15

2.1 Parameters of the CMS Solenoid Magnet . . . . . . . . . . . . . . . . . 28

$2.2 \quad$ Scintillator tile and brass alloy thickness for each layer in an HB wedge [4]. $\quad 37$

$4.1 \hat{p_{T}}$ ranges and cross sections of the official MC generated events for the CMS collaboration used to model photons for the analysis. . . . . . . . . . . 53

$4.2 \hat{p_{T}}$ ranges and cross sections of the official MC generated events for the CMS collaboration used to model background for the analysis. . . . . . . . . . 53

4.3 The weights used to account for pileup according to the number of primary vertices a MC event reports. . . . . . . . . . . . . . . . . . . 65

4.42011 Datasets used for this analysis. The effective luminosity reflects the amount of data collected from the trigger used in the analysis. . . . . . . 66

4.5 Additional correction factors for the isolation variables used in the analysis. The value of $\rho_{\text {event }}$ is obtained on an event-by-event basis. These values only reflect candidates in EB. . . . . . . . . . . . . . . . . . . . . 66

4.6 Photon identification efficiencies and it's corresponding uncertainty in bins

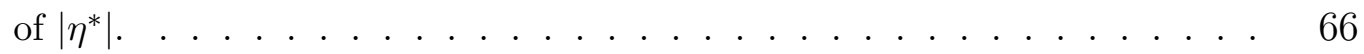

5.1 Fraction of candidates below $\sigma_{i \eta i \eta}=0.01$ for data, signal and background along with their uncertainties. . . . . . . . . . . . . . . . . . 72

5.2 Purity and uncertainty for each $\left|\eta^{*}\right| . \ldots \ldots \ldots$. . . . . . . . . 73

5.3 The purity uncertainty corrected, $\Delta P_{\text {corr }}$, using the correction factor, $\sigma$, reported by the pull distributions shown in Appendix $|\mathrm{F}|$. . . . . . . . . 74

6.1 Fraction of candidates with $\sigma_{i \eta i \eta}<0.01$ for QCD MC candidates selected using photon identification cuts, QCD MC candidates selected using sbs cuts and data selected using sbs cuts along with their respective uncertainty. 77 
6.2 Purities calculated using three background templates obtained from QCD MC candidates selected with photon identification cuts, QCD MC candidates selected with sbs cuts and data selected with sbs cuts along with their respective uncertainty. . . . . . . . . . . . . . . . . . . . . . . 78

6.3 Number of entries in $\left|\eta^{*}\right|$ using the background templates obtained from QCD MC along with the systematic uncertainty due to the background shape. 79

6.4 Systematic uncertainties for this analysis. . . . . . . . . . . . . 80

7.1 Theoretical uncertainty due to varying the factorization scale, $\mu_{F}$, and the renormalization scale, $\mu_{R}$. . . . . . . . . . . . . . . . . . . 84

7.2 Values for the number of candidates $(N)$, and the relative statistical uncertainties due to the number of candidates $\left(\frac{\Delta N}{N}\right)$, purity $\left(\frac{\Delta P}{P}\right)$ and efficiency $\left(\frac{\Delta \epsilon}{\epsilon}\right)$ for each bin in $\left|\eta^{*}\right|$. Also included are the total uncorrelated $\left(\Delta_{\text {uncorr }}\right)$ and correlated $\left(\Delta_{\text {corr }}\right)$ uncertainties. . . . . . . . . . . . . . . 85 
1.1 Experimental results from Robert Hofstadtler's electron scattering experiments where it was discovered that protons are composite structures, shown by the data points deviating from line (c) [5]. . . . . . . . . . . . . 2

1.2 The Standard Model of Particle Physics. . . . . . . . . . . . . . . . . . . 5

1.3 The invariant mass distributions for two decay modes for the Higgs boson, where the Higgs decays to two photons $(H \rightarrow \gamma \gamma)$ on the left and where the Higgs decays to two $Z^{0}$ bosons, which then decay into four leptons $(H \rightarrow Z Z \rightarrow l l l l)$ on the right. Both distributions show an excess of events at $\mathrm{m} \sim 125 \mathrm{GeV}$. Data collected during the 2011 and 2012 runs are used for both distributions. . . . . . . . . . . . . . . . . . 6

1.4 Measured values of $\alpha_{s}$ as a function of the energy scale $Q[6]$. . . . . . . . $\quad 9$

1.5 Schematic view of $p p$ collision. Parton $a$ from proton $A$ interacts with parton $b$ from proton $B$ to form the hard scatter. One of the outgoing partons fragments and forms a jet. . . . . . . . . . . . . . . . . . 10

1.6 Parton distribution functions of quarks and gluons as a function of their fractional momentum, $x$, when the scale is $\mu^{2}=10 \mathrm{GeV}^{2}$ (left) and when the

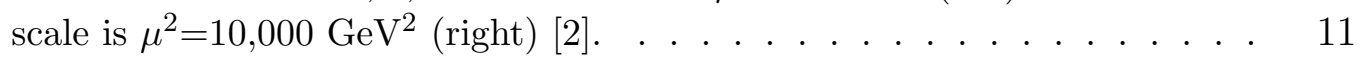

1.7 Collision in the CM frame (left) and in the boosted frame (right). . . . . . 12

1.8 The $\left|\cos \theta^{*}\right|$ distributions from the $\mathrm{D} \varnothing$ collaboration [7]. The solid black line is the NLO prediction. The dotted line represents Rutherford scattering. The Data and theory agree. . . . . . . . . . . . . . . . . . . . 13

1.9 The $\left|\cos \theta^{*}\right|$ distribution from the CDF collaboration [8]. The open circles represent the angluar distribution in $\gamma+$ Jet events, which show good agreement with its NLO prediciton represented by the solid line. A factor of $\frac{1}{N}$ is missing in the y-axis labeling. . . . . . . . . . . . . . . . . . . . 14

1.10 Leading order feynman diagrams for prompt photon production. Top dia\begin{tabular}{|l|}
\hline grams show the $q \rightarrow g \gamma$ feynman diagrams at leading order. Middle diagrams \\
\hline \hline shows the $q g \rightarrow g \gamma$ process for the t-channel(left) and the s-channel (right) \\
\hline \hline at leading order. The feynman diagram for fragmentation photon produc- \\
\hline \hline tion at leading order is shown at the bottom. The vertex in which the \\
\hline \hline fragmented photon is present contributes a factor of $\frac{\alpha}{\alpha_{s}}$, making the process \\
\hline of the order $\alpha \alpha_{s} . \quad \ldots \ldots \ldots \ldots$ \\
\hline
\end{tabular} 1.11 NLO Feynman diagrams for QCD Compton scattering. Adpated from [9]. 18 
1.12 NLO Feynman diagrams for quark annihilation. Adapted from [9]. . . . .

1.13 One-loop Feynman diagrams for prompt photon production. Adapted from [9] 20

1.14 Single jet invariant cross section as a function of the cut-off parameter $\delta_{s}$ while keeping $\delta_{c}$ constant. The one-loop contribution is shown on the left, the 3-body final state contribution is shown on the middle and the combined result is shown on the right [10]. . . . . . . . . . . . . . . . . . . 21

$2.1 \quad$ Pictorial representation of CERN's accelerator complex, the LHC ring and the experimental detectors [11]. . . . . . . . . . . . . . . . . . . . 24

2.2 The Compact Muon Solenoid Detector [4]. . . . . . . . . . . . . . . . . 26

2.3 Quarter view of the CMS tracking system [4]. The tracking system consists of a combination of microstrip silicon detectors and pixel silicon detectors in the barrel (horizontal lines) and the endcap regions (vertical lines). . . . 30

2.4 Artist rendering of ECAL. The EB modules are horizontal in the central region of ECAL. Each endcap is comprised of 2 'Dees', which get their name from the D-like shape it resembles [12]. . . . . . . . . . . . . . . . 34

2.5 Off-pointing geometry for the EB supermodules. The circle has a radius of $66.7 \mathrm{~mm} \mathrm{[12].} \mathrm{.} \mathrm{.} \mathrm{.} \mathrm{.} \mathrm{.} \mathrm{.} \mathrm{.} \mathrm{.} \mathrm{.} \mathrm{.} \mathrm{.} \mathrm{.} \mathrm{.} \mathrm{.} \mathrm{.} \mathrm{.} \mathrm{.} \mathrm{.} \mathrm{.} \mathrm{.} \mathrm{.} \mathrm{.} \mathrm{.} \mathrm{.} \mathrm{.} \mathrm{.} \mathrm{.} \mathrm{.} \mathrm{.} \mathrm{.} \mathrm{.} 34$

2.6 Measured ECAL resolution from a test beam [4]. . . . . . . . . . . . . . 36

$2.7 \quad$ Schematic of HB and HE towers. HB and HE overlap at tower 16 [4]. . . . 38

2.8 CMS Tier organization. . . . . . . . . . . . . . . . . . . . . . . . . . 42

3.1 Monte Carlo generated particles with soft particles surrounding hard particles using 4 jet finding algorithms [13]. . . . . . . . . . . . . . . 50

3.2 Jet Energy Correction Factor, $\mathrm{E}_{\text {corrected }}$, as a function of Jet $\eta$ for CALO jets, Jet-Plus-Track Jets (JPT) and Particle Flow Jets (used in this analy-

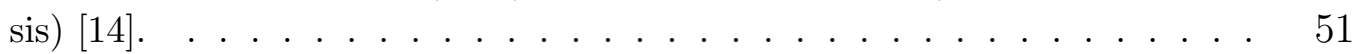

4.1 Number of primary vertices in data and signal MC (left). The Number of primary vertices in signal MC after corrected for pileup (right). . . . . . . 54

4.2 The total integrated luminosity delivered (blue) and recorded (yellow) during the 2011 run [15]. . . . . . . . . . . . . . . . . . . . . . 55

4.3 Visual depiction of an isolation cone. . . . . . . . . . . . . . . . . 58 
4.4 Distributions of the following photon candidate kinematic variables in which cuts are made to select signal candidates: Ecal Isolation on the upper-left, Hcal Isolation on the upper-right side, Hollow Cone Track Isolation on the bottom. . . . . . . . . . . . . . . 59

4.5 The $p_{T}$ distribution of photon candidates (left) and the $\eta^{\gamma}$ distribution (right) for photon candidates with $0.2<\left|\eta^{*}\right|<0.3$. . . . . . . . . . . . 60

4.6 The $p_{T}$ distribution of jets (left) and $\eta^{\text {jet }}$ distribution (right) for jet candidates with $0.2<\left|\eta^{*}\right|<0.3 . \quad \ldots \ldots \ldots$. . . . . . . . . . 61

4.7 Level 1 trigger efficiency as a function of $E_{T}[16] . \ldots 2$

4.8 HLT efficiency as a function of $p_{T} . \ldots \ldots \ldots$

4.9 Photon identification efficiencies for each individual cut as well as the combined set of cuts. . . . . . . . . . . . . . . . . . . . . . . . . 64

5.1 Signal (striped histogram) and background (white histogram) templates with data overlaid (crosses) for $\sigma_{i \eta i \eta}$, the discriminant variable with 0.30 $<\left|\eta^{*}\right|<0.40$. Bottom shows the area under the curve of the top plot is calculated for every point in $\sigma_{i \eta i \eta}$ for signal MC (squares), background (triangles) and data (circles). . . . . . . . . . . . . . . . . . . 68

5.2 The $\sigma_{\text {ini }}$ template distribution for QCD MC with photon Identification cuts (striped histogram), QCD MC with SBS cuts (dotted line) and data with SBS cuts (circles) on the right and the area under the $\sigma_{i \eta i \eta}$ distribution for QCD MC with photon Identification cuts (closed triangles), QCD MC with SBS cut (open triangles) and Data SBS (open circles) on the right. Directly below the area plot is the ratio between QCD MC with photon Identification cuts to Data with SBS cuts (closed triangles) and the ratio of QCD MC with SBS cuts to data with SBS cuts (open triangles). . . . . . 69

5.3 The $\sigma_{i \eta i \eta}$ Vs. IsoTRK 2-dimensional distribution for 2010 data. The plane is divided into four regions labeled A, B, C and D. . . . . . . . . . . . . 70

5.4 Fraction of candidates in the signal, background and data samples with $\sigma_{i \eta i \eta}$ $<0.01$ as a function of $\left|\eta^{*}\right| . \mid \ldots \ldots \ldots \ldots$. . . . . . . . . . . 71

5.5 Purity as a function of $\left|\eta^{*}\right|$. The uncertainties shown are calculated using Eq.|5.4. . . . . . . . . . . . . . . . . . . 75

5.6 Pull distribution for candidates from the pseudo experiments generated with $0.4<\left|\eta^{*}\right|<0.5$ fitted with a Gaussian function. The mean for the pull distribution is centered at 0 while the width $(\sigma)$ is less than $1 . \quad$. . . . . 75 
6.1 The fraction of candidates below $\sigma_{i \eta i \eta}=0.01$ for QCD MC with photon identification cuts (solid triangles), QCD MC selected with SBS cuts (open triangles) and data selected with SBS cuts (solid circles) on the left. The purity as a function of $\left|\eta^{*}\right|$ for QCD MC with photon identification cuts (solid triangles), QCD MC selected with SBS cuts (open triangles) and Data selected with SBS cuts (solid circles) on the right. Both plots give similar results for each background template. . . . . . . . . . . . . . . 76

6.2 Plot showing $\left|\eta^{*}\right|$ using two different background templates; Data selected with SBS cuts and QCD MC selected with photon identification cuts on top. The bottom plot is the ratio between the two results, taken as the systematic uncertainty of the background shape. The largest deviation between data and both set of cuts used on QCD is seen in the highest $\left|\eta^{*}\right|$ bin. . . . . . 80

6.3 Angular distribution $\left|\eta^{*}\right|$ using the original $\eta^{\text {jet }}$ in black solid circles and the smeared $\eta^{\text {jet }}$ in the black open circles. Below is the ratio between the two distributions showing that the jet resolution does not affect the $\left|\eta^{*}\right|$ distribution by much. . . . . . . . . . . . . . . . . . . . . 81

7.1 The top plot shows the angular distribution $\left|\eta^{*}\right|$ using three different sets of values for $\mu_{F}$ and $\mu_{F}$, which represent the factorization scale and renormalization factor, respectively. These values are traditionally taken to be equal to the $p_{T}$ of the photon. Below is the ratio between the varied and traditional values of the scales. These differences are taken as the theoretical uncertainty. . . . . . . . . . . . . . . . . . . 83

7.2 The angular distribution, $\left|\eta^{*}\right|$, for 2011 data is represented by the black circles where the error bars represent the combined correlated and uncorrelated uncertainties. The histogram represents the NLO QCD prediction along with its weighted statistical uncertainty. The distribution shown below is the data/theory ratio, showing that data agrees with NLO predictions with the exception of the last $\left|\eta^{*}\right|$ bin. The striped distribution in the ratio plot is the theoretical uncertainty values shown in Table 7.1$]$. . . . . . . 89

7.3 The $\left|\eta^{*}\right|$ distribution for data (closed circles) overlaid with three NLO predictions for $\left|\eta^{*}\right|$; Owens (solid-dotted line), SHERPA (solid line) and MADGRAPH (dotted line) for the exclusive case in which only one jet accompanies a photon in an event. . . . . . . . . . . . . . . . . . . . . . . . . . . . . . 90

7.4 Angular distribution in Z+jet events for the lepton channel $(\rightarrow e e$ and $\rightarrow \mu \mu$ ) which shows a discrepancy between the MADGRAPH and SHERPA NLO theoretical predictions and the reference NLO theory prediction (MCFM). 
7.5 Representation of one parton shower forming two jets on the left side. The right side depicts each parton shower forming an individual jet. The matching parameter avoids double counting of parton showers in cases like the left side example where more than one jet may be formed by the same parton shower. . . . . . . . . . . . . . . . . . 91

A.1 The leading photon's $p_{T}$ and $\eta$ distributions (top) and the leading jet's $p_{T}$ and $\eta$ distributions on bottom for candidates with $0.0<\left|\eta^{*}\right|<0.1$. . . . . . 97

A.2 The leading photon's $p_{T}$ and $\eta$ distributions (top) and the leading jet's $p_{T}$ and $\eta$ distributions on bottom for candidates with $0.1<\left|\eta^{*}\right|<0.2$. . . . . . 98

A.3 The leading photon's $p_{T}$ and $\eta$ distributions (top) and the leading jet's $p_{T}$ and $\eta$ distributions on bottom for candidates with $0.2<\left|\eta^{*}\right|<0.3$. . . . . . 99

A.4 The leading photon's $p_{T}$ and $\eta$ distributions (top) and the leading jet's $p_{T}$ and $\eta$ distributions on bottom for candidates with $0.3<\left|\eta^{*}\right|<0.4$. . . . . . 100

A.5 The leading photon's $p_{T}$ and $\eta$ distributions (top) and the leading jet's $p_{T}$ and $\eta$ distributions on bottom for candidates with $0.4<\left|\eta^{*}\right|<0.5$. . . . . . 101

A.6 The leading photon's $p_{T}$ and $\eta$ distributions (top) and the leading jet's $p_{T}$ and $\eta$ distributions on bottom for candidates with $0.5<\left|\eta^{*}\right|<0.6$. . . . . . 102

A.7 The leading photon's $p_{T}$ and $\eta$ distributions (top) and the leading jet's $p_{T}$ and $\eta$ distributions on bottom for candidates with $0.6<\left|\eta^{*}\right|<0.7$. . . . . . 103

A.8 The leading photon's $p_{T}$ and $\eta$ distributions (top) and the leading jet's $p_{T}$ and $\eta$ distributions on bottom for candidates with $0.7<\left|\eta^{*}\right|<0.8$. . . . . . 104

A.9 The leading photon's $p_{T}$ and $\eta$ distributions (top) and the leading jet's $p_{T}$ and $\eta$ distributions on bottom for candidates with $0.8<\left|\eta^{*}\right|<0.9$. . . . . . 105

A.10 The leading photon's $p_{T}$ and $\eta$ distributions (top) and the leading jet's $p_{T}$ and $\eta$ distributions on bottom for candidates with $0.9<\left|\eta^{*}\right|<1.0$. . . . . . 106

A.11 The leading photon's $p_{T}$ and $\eta$ distributions (top) and the leading jet's $p_{T}$ and $\eta$ distributions on bottom for candidates with $1.0<\left|\eta^{*}\right|<1.1$. . . . . . 107

A.12 The leading photon's $p_{T}$ and $\eta$ distributions (top) and the leading jet's $p_{T}$ and $\eta$ distributions on bottom for candidates with $1.1<\left|\eta^{*}\right|<1.2$. . . . . . 108

A.13 The leading photon's $p_{T}$ and $\eta$ distributions (top) and the leading jet's $p_{T}$ and $\eta$ distributions on bottom for candidates with $1.2<\left|\eta^{*}\right|<1.3$. . . . . . 109

A.14 The leading photon's $p_{T}$ and $\eta$ distributions (top) and the leading jet's $p_{T}$ and $\eta$ distributions on bottom for candidates with $1.3<\left|\eta^{*}\right|<1.4$. . . . . . 110 
C.1 The $\sigma_{i \eta i \eta}$ distribution for photon candidates with $0.0<\left|\eta^{*}\right|<0.1$ for signal, background and data. . . . . . . . . . . . . . . . . . . 113

C.2 The $\sigma_{i \eta i \eta}$ distribution for photon candidates with $0.1<\left|\eta^{*}\right|<0.2$ for signal, background and data. . . . . . . . . . . . . . . . . . . . . 113

C.3 The $\sigma_{i \eta i \eta}$ distribution for photon candidates with $0.2<\left|\eta^{*}\right|<0.3$ for signal, background and data. . . . . . . . . . . . . . . . . . . . . 114

C.4 The $\sigma_{i \eta i \eta}$ distribution for photon candidates with $0.3<\left|\eta^{*}\right|<0.4$ for signal, background and data. . . . . . . . . . . . . . . . . . . . . . 114

C.5 The $\sigma_{i \eta i \eta}$ distribution for photon candidates with $0.4<\left|\eta^{*}\right|<0.5$ for signal, background and data. . . . . . . . . . . . . . . 115

C.6 The $\sigma_{\text {ini } i}$ distribution for photon candidates with $0.5<\left|\eta^{*}\right|<0.6$ for signal, background and data. . . . . . . . . . . . . . . . . 115

C.7 The $\sigma_{i \eta i \eta}$ distribution for photon candidates with $0.6<\left|\eta^{*}\right|<0.7$ for signal, background and data . . . . . . . . . . . . . . . 116

C.8 The $\sigma_{i \eta i \eta}$ distribution for photon candidates with $0.7<\left|\eta^{*}\right|<0.8$ for signal, background and data. . . . . . . . . . . . . . . 116

C.9 The $\sigma_{\text {ini }}$ distribution for photon candidates with $0.8<\left|\eta^{*}\right|<0.9$ for signal, background and data. . . . . . . . . . . . . . . . . . . . . 117

C.10 The $\sigma_{i \eta i \eta}$ distribution for photon candidates with $0.9<\left|\eta^{*}\right|<1.0$ for signal, background and data. . . . . . . . . . . . . . . . . . 117

C.11 The $\sigma_{i \eta i \eta}$ distribution for photon candidates with $1.0<\left|\eta^{*}\right|<1.1$ for signal, background and data. . . . . . . . . . . . . . . . . . . . . . . 118

C.12 The $\sigma_{i \eta i \eta}$ distribution for photon candidates with $1.1<\left|\eta^{*}\right|<1.2$ for signal, background and data. . . . . . . . . . . . . . . . . 118

C.13 The $\sigma_{i \eta i \eta}$ distribution for photon candidates with $1.2<\left|\eta^{*}\right|<1.3$ for signal, background and data. . . . . . . . . . . . . . . . . . . . . . . . 119

C.14 The $\sigma_{i \eta i \eta}$ distribution for photon candidates with $1.3<\left|\eta^{*}\right|<1.4$ for signal, background and data. . . . . . . . . . . . . . . . . . . . . . 119

D.1 The $\sigma_{i \eta i \eta}$ distribution for photon candidates with $0.0<\left|\eta^{*}\right|<0.1$ for the various background shapes used in this analysis. . . . . . . . . . . . . . . 120

D.2 The $\sigma_{i \eta i \eta}$ distribution for photon candidates with $0.1<\left|\eta^{*}\right|<0.2$ for the various background shapes used in this analysis. . . . . . . . . . . . . . . 121 
D.3 The $\sigma_{\text {ini }}$ distribution for photon candidates with $0.2<\left|\eta^{*}\right|<0.3$ for the various background shapes used in this analysis. . . . . . . . . . . . . . . 121

D.4 The $\sigma_{i \eta i \eta}$ distribution for photon candidates with $0.3<\left|\eta^{*}\right|<0.4$ for the various background shapes used in this analysis. . . . . . . . . . . . . . . 122

D.5 The $\sigma_{\text {inin }}$ distribution for photon candidates with $0.4<\left|\eta^{*}\right|<0.5$ for the various background shapes used in this analysis. . . . . . . . . . . . . 122

D.6 The $\sigma_{\text {ini }}$ distribution for photon candidates with $0.5<\left|\eta^{*}\right|<0.6$ for the various background shapes used in this analysis. . . . . . . . . . . . . . . 123

D.7 The $\sigma_{i \eta i \eta}$ distribution for photon candidates with $0.6<\left|\eta^{*}\right|<0.7$ for the various background shapes used in this analysis. . . . . . . . . . . . . . 123

D.8 The $\sigma_{\text {ini }}$ distribution for photon candidates with $0.7<\left|\eta^{*}\right|<0.8$ for the various background shapes used in this analysis. . . . . . . . . . . . . . . 124

D.9 The $\sigma_{\text {ini }}$ distribution for photon candidates with $0.8<\left|\eta^{*}\right|<0.9$ for the various background shapes used in this analysis. . . . . . . . . . . . . . . 124

D.10 The $\sigma_{i \eta i \eta}$ distribution for photon candidates with $0.9<\left|\eta^{*}\right|<1.0$ for the various background shapes used in this analysis. . . . . . . . . . . . . . 125

D.11 The $\sigma_{\text {ini }}$ distribution for photon candidates with $1.0<\left|\eta^{*}\right|<1.1$ for the various background shapes used in this analysis. . . . . . . . . . . . . . . 125

D.12 The $\sigma_{\text {ini }}$ distribution for photon candidates with $1.1<\left|\eta^{*}\right|<1.2$ for the various background shapes used in this analysis. . . . . . . . . . . . . . . 126

D.13 The $\sigma_{\text {ini }}$ distribution for photon candidates with $1.2<\left|\eta^{*}\right|<1.3$ for the various background shapes used in this analysis. . . . . . . . . . . . . . 126

D.14 The $\sigma_{\text {ini }}$ distribution for photon candidates with $1.3<\left|\eta^{*}\right|<1.4$ for the various background shapes used in this analysis. . . . . . . . . . . . . . . 127

E.1 The 2-dimensional plane in which the variables used for estimating background in data is defined. The plane is broken up into 4 sections; A, B, C, and D. . . . . . . . . . . . . . . . . . . . . 129

F.1 Purity pull distribution for candidates in pseudo data with $0.0<\left|\eta^{*}\right|<0.1$. 131

F.2 $\quad$ Purity pull distribution for candidates in pseudo data with $0.1<\left|\eta^{*}\right|<0.2$. 132

F.3 $\quad$ Purity pull distribution for candidates in pseudo data with $0.2<\left|\eta^{*}\right|<0.3$. 132

F.4 Purity pull distribution for candidates in pseudo data with $0.3<\left|\eta^{*}\right|<0.4$. 133

F.5 Purity pull distribution for candidates in pseudo data with $0.4<\left|\eta^{*}\right|<0.5$. 133 
F.6 $\quad$ Purity pull distribution for candidates in pseudo data with $0.5<\left|\eta^{*}\right|<0.6$. 134

F.7 $\quad$ Purity pull distribution for candidates in pseudo data with $0.6<\left|\eta^{*}\right|<0.7$. 134

F.8 $\quad$ Purity pull distribution for candidates in pseudo data with $0.7<\left|\eta^{*}\right|<0.8$. 135

F.9 $\quad$ Purity pull distribution for candidates in pseudo data with $0.8<\left|\eta^{*}\right|<0.9$. 135

F.10 Purity pull distribution for candidates in pseudo data with $0.9<\left|\eta^{*}\right|<1.0$. 136

F.11 Purity pull distribution for candidates in pseudo data with $1.0<\left|\eta^{*}\right|<1.1$. 136

F.12 Purity pull distribution for candidates in pseudo data with $1.1<\left|\eta^{*}\right|<1.2$. 137

F.13 Purity pull distribution for candidates in pseudo data with $1.2<\left|\eta^{*}\right|<1.3$. 137

F.14 Purity pull distribution for candidates in pseudo data with $1.3<\left|\eta^{*}\right|<1.4$. 138

G.1 The 2-D representation of the response matrix. . . . . . . . . . . . . . . 140

G.2 Results for all four unfolding techniques, the upper left distribution was unfolded using the D'Augositini unfolding technique, the upper right distribution was unfolded using the Bin-by-Bin technique, the lower left distribution was unfolded using the Singular Value Decomposition (SVD) technique, and the lower right distribution was unfolded using the matrix inversion technique. All unfolded results vary by less than $1 \%$ from their measured distributions, as shown by the ratio plots located directly beneath each distribution. . . . . . . . . . . . . . . . 143

H.1 Angular distribution for the nominal (black), loose (green) and tight (blue) isolation criteria overlaid with NLO theory predictions. . . . . . . . . . . 144

H.2 Angular distribution when isolation is tight (left) and when isolation is loose (right). Both figures have their NLO theoretical prediction overlaid as well as the theory/data ratio (below) showing good agreement. . . . . . . . . 145

I.1 The template distribution $\sigma_{i \eta i \eta}$ for different number of primary vertices. The shape of the distribution shows no sign of change with different number of primary vertices. . . . . . . . . . . . . . . . . . . . . . . 147

I.2 The mean value of each $\sigma_{i \eta i \eta}$ distribution as a function of number of primary vertices. The mean is constant. . . . . . . . . . . . . . . . . . . . 147

I.3 The RMS of each $\sigma_{i \eta i \eta}$ distribution as a function of number of primary vertices. The RMS is constant. . . . . . . . . . . . . . . . . . . . . 148

J.1 The angular distribution, with $f_{b k g}$ corrected which affects the purity term in the angular distribution expression and hence the overall distribution. . 150 


\section{CHAPTER 1}

\section{THEORY}

From the time of Ernest Rutherford and his famous scattering experiments in 1911, measuring the angular distribution has been a vital technique in determining the structure of matter. Prior to the Rutherford scattering experiments, the accepted model of the atom was developed by J.J. Thompson. Thompson's plum pudding model stated that electrons (represented by the plums) were uniformly distributed within a positively charged sphere (the pudding). Under Rutherfords guidance, Hans Geiger and Ernest Marsden shot an $\alpha$ particle beam (helium nuclei), which is positively charged, onto gold foil [17]. Had the plum pudding model of atoms been correct, Rutherford's scattering experiment would have shown that the angle of deflection between the $\alpha$ particle and the gold foil was small. Instead, they observed the $\alpha$ particles from the gold foil deflected at large scattering angles, proving that the mass of positively charged atoms are focused at the center and not distributed throughout the atom as predicted by the plum pudding model of the atom. The large angle of deflection was later shown to be caused by the Coulombic force, the force charged particles exert on each other as they approach. Rutherford's experiment showed how measuring the angular distribution gave insight into the atomic structure.

Further understanding of the atomic structure continued in the 1950's with the work of Robert Hofstadter and his electron scattering experiments which studied the charge and magnetic moment distributions within protons and neutrons. The electronscattering experiments determined significant information about nuclei including their size and surface thickness parameters [5, 18, 19, 20]. 


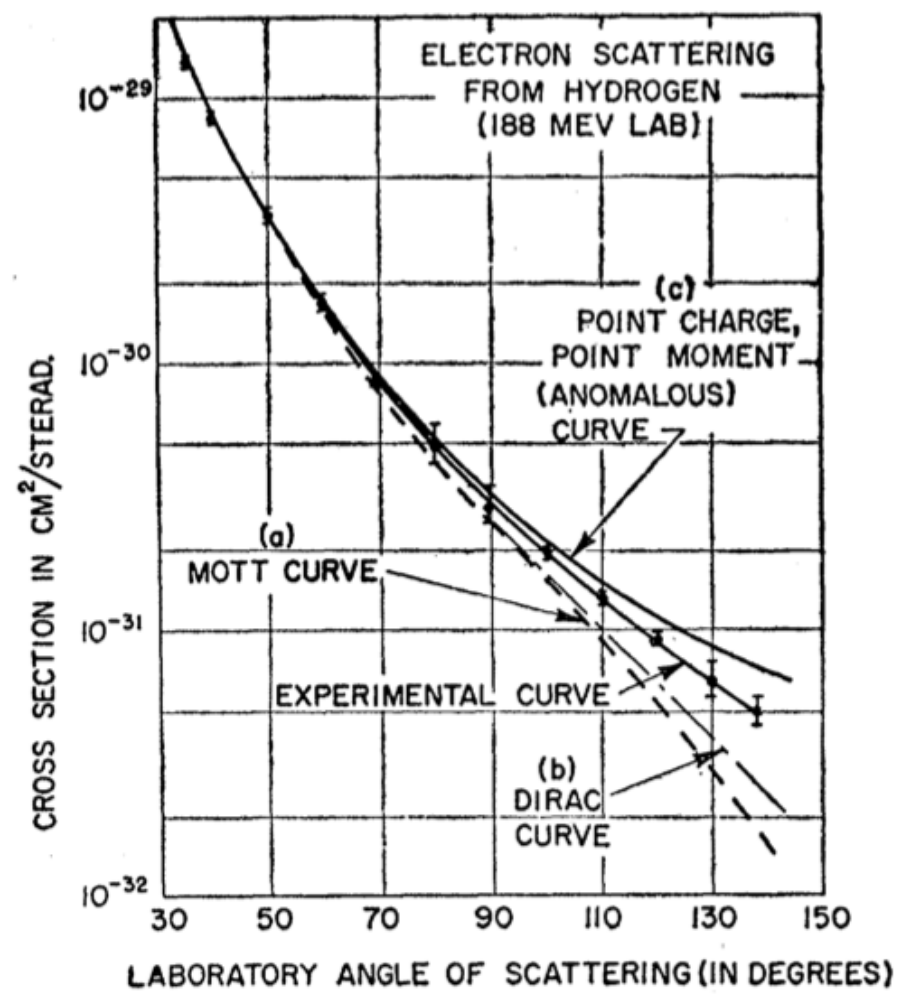

Figure 1.1: Experimental results from Robert Hofstadtler's electron scattering experiments where it was discovered that protons are composite structures, shown by the data points deviating from line (c) [5].

In the late 1960's a team of scientists at Stanford's Linear Accelerator Center (SLAC) found that protons, one of the particles that make up an atom's nucleus, consisted of even smaller particles. A deviation in the scattering angle showed the proton's sub-structure similarly to how Rutherford showed the substructure of a gold atom back in 1911. This laid the foundation for what is known as 'The Standard Model' of particle physics which encompasses the sub-atomic particles that make up all known matter in the universe.

\subsection{The Standard Model}

The Standard Model is the theory of particle physics; it encompasses all fundamental particles that make up matter and its interactions. Of the four known forces, three are included in the Standard Model (weak, electromagnetic and strong forces). 
The gravitational force plays an insignificant role in the fundamental interactions of particles and thus is not included in the Standard Model[21].

The 12 fundamental particles in the Standard Model have spin $=\frac{1}{2}$, indicating they are all fermions. These 12 particles are comprised of quarks and leptons; their properties are shown in Table 1.1 [22, 1]. For each quark(lepton) their exist an anti-quark(antilepton) with the same characteristics, except with opposite charges. For example, the up quark (denoted as $u$ ) has an electric charge of $q=\frac{2}{3}$ while the anti-up quark (denoted as $\bar{u}$ ) has $q=-\frac{2}{3}$. Quarks and leptons are grouped into three generations. The first generation is comprised of particles that make up most ordinary matter while the second and third generations consists of heavier fermions [23, 3].

Fermions that interact only via the electromagnetic or weak forces are known as leptons. The first lepton to be discovered, and the most widely known is the electron (e). Its neutrally charged partner, the electron neutrino $\left(\nu_{e}\right)$ is significantly lighter and is more difficult to detect experimentally. The remaining leptons, the muon $(\mu)$ and the tau $(\tau)$ also have their respective neutrino partners $\nu_{\mu}$ and $\nu_{\tau}$. As with charged leptons, neutrinos also have their own anti-particles $\overline{\nu_{e}}, \overline{\nu_{\mu}}$ and $\overline{\nu_{\tau}}$.

Fermions that interact via the strong force are quarks. Analogous to electric charge, quarks also have a color charge, which contrary to its name does not reflect its visual representation but rather an intrinsic property of the particle that couples it to the force carriers exchanged between quarks. There are three such 'color' charges: red, green and blue. Their anti-color equivalents are anti-red, anti-green and anti-blue. A group of three quarks may combine to form a baryon. The most notable baryons are the proton and neutron with a quark configuration of $u u d$ and $d d u$ respectively. All three quarks making up the baryon must combine such that the baryon is 'colorless', which can be accomplished analogously to how white light is produced; each quark 
must have a color of red, blue or green. Quarks may also combine to form mesons, another composite particle made up of a quark and an anti-quark. An example of a meson is $\pi^{+}(u \bar{d})$. An example of mesons achieving a colorless state by having one red quark and one anti-red anti-quark [24].

Table 1.1: Table of partons and their properties [1].

\begin{tabular}{|c|c|c|c|c|}
\hline Generation & Particle $($ symbol) & Lepton Number & Charge $(e)$ & Mass $(\mathrm{MeV})$ \\
\hline First Generation & $\operatorname{up}(u)$ & 0 & $2 / 3$ & 5 \\
& $\operatorname{down}(d)$ & 0 & $-1 / 3$ & 10 \\
& electron $(e)$ & $L_{e}=1$ & -1 & .5110 \\
& electron neutrino $\left(\nu_{e}\right)$ & $L_{e}=-1$ & 0 & $<7 \times 10^{-6}$ \\
\hline Second Generation & charm $(c)$ & 0 & $2 / 3$ & 1500 \\
& strange $(s)$ & 0 & $-1 / 3$ & 150 \\
& muon $(\mu)$ & $L_{\mu}=1$ & -1 & 105.7 \\
& muon neutrino $\left(\nu_{\mu}\right)$ & $L_{\mu}=-1$ & 0 & $<.27$ \\
\hline Third Generation & $\operatorname{top}(t)$ & 0 & $2 / 3$ & 175 \\
& $\operatorname{bottom}(b)$ & 0 & $-1 / 3$ & 5000 \\
& $\operatorname{tau}(\tau)$ & $L_{\tau}=1$ & -1 & 1777 \\
& $\operatorname{tau} \operatorname{neutrino}\left(\nu_{\tau}\right)$ & $L_{\tau}=-1$ & 0 & $<33$ \\
\hline
\end{tabular}

Each force in the Standard Model plays a specific role and is responsible for the behavior observed during particle interactions. Each force has a mediating particle whose role is that of force exchange between interacting particles. The strong force is responsible for quark interactions. Gluons are exchanged between quarks, keeping them bound together inside baryons and mesons. The electromagnetic force is responsible for keeping protons and electrons inside atoms through the exchange of a photon. The weak force is responsible for decays of heavy quarks and leptons in which one of the massive vector bosons, $Z^{0}$ or $W^{ \pm}$, are exchanged.

It was first theorized, and later experimentally shown, that the electromagnetic and weak forces are manifistations of the same force, known as the electroweak force [25]. The largest difference between the electromagnetic and weak forces is their mediating particles. The electromagnetic mediator, the photon, is massless while the weak 


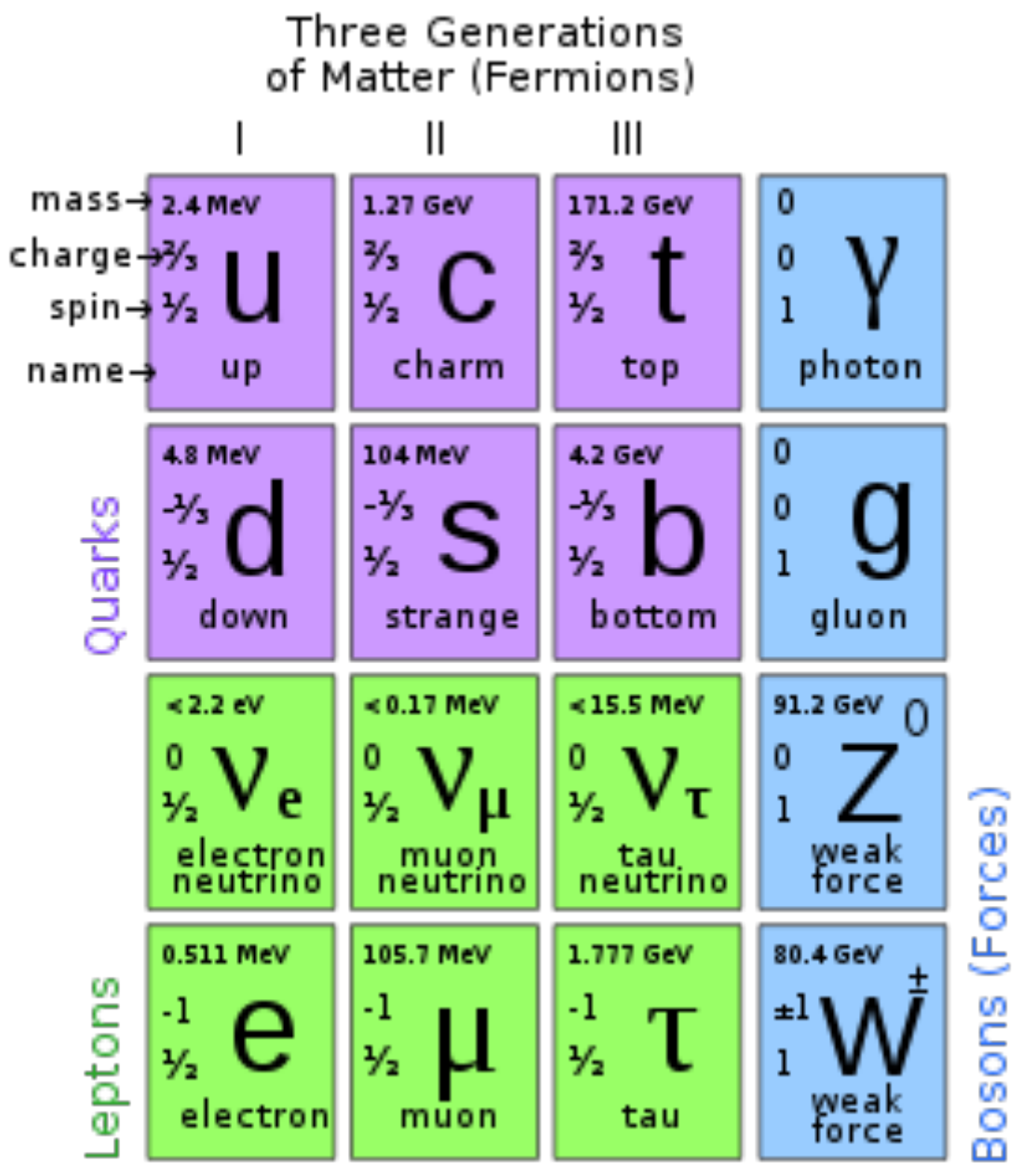

Figure 1.2: The Standard Model of Particle Physics.

mediators, the $W^{ \pm}$and $Z^{0}$, have mass (values shown on Table 1.2 ). The observed difference in mass between these force carriers is explained through spontaneous symmetry breaking (also known as the Higgs mechanism) [23], in which a Higgs field is produced and interacts with the $W^{ \pm}$and $Z^{0}$ resulting in these bosons acquiring mass [26].

The Higgs boson is the quantum of the Higgs field and until recently was the missing piece in the Standard Model of particle physics. Various accelerator experiments have taken on the arduous task in attempting to find evidence for the existence of the 
Table 1.2: Table of forces and their mediating boson particles [2.

\begin{tabular}{|c|c|c|c|}
\hline Force & Mediators $($ symbol) & Charge & Mass $(\mathrm{GeV})$ \\
\hline Strong & gluons $(g)$ & 0 & 0 \\
Electroweak & photons $(\gamma)$ & 0 & 0 \\
& $Z^{0}$ & 0 & $91.1876 \pm 0.0021$ \\
& $W^{ \pm}$ & \pm 1 & $80.398 \pm 0.023$ \\
\hline
\end{tabular}

Higgs, however, it is the experiments at the Large Hadron Collider (LHC) at CERN which have recently discovered a new boson which has the same characteristics that the Higgs boson is theorized to have [27]. The distributions in Fig. 1.3 show the invariant mass for two decay modes of the Higgs boson, $H \rightarrow \gamma \gamma$ and $H \rightarrow Z Z \rightarrow l l l l$ in which both distributions show an excess in the mass range $\sim 125 \mathrm{GeV}$, which lies within the mass window region obtained most recently by the Compact Muon Solenoid experiment [28].
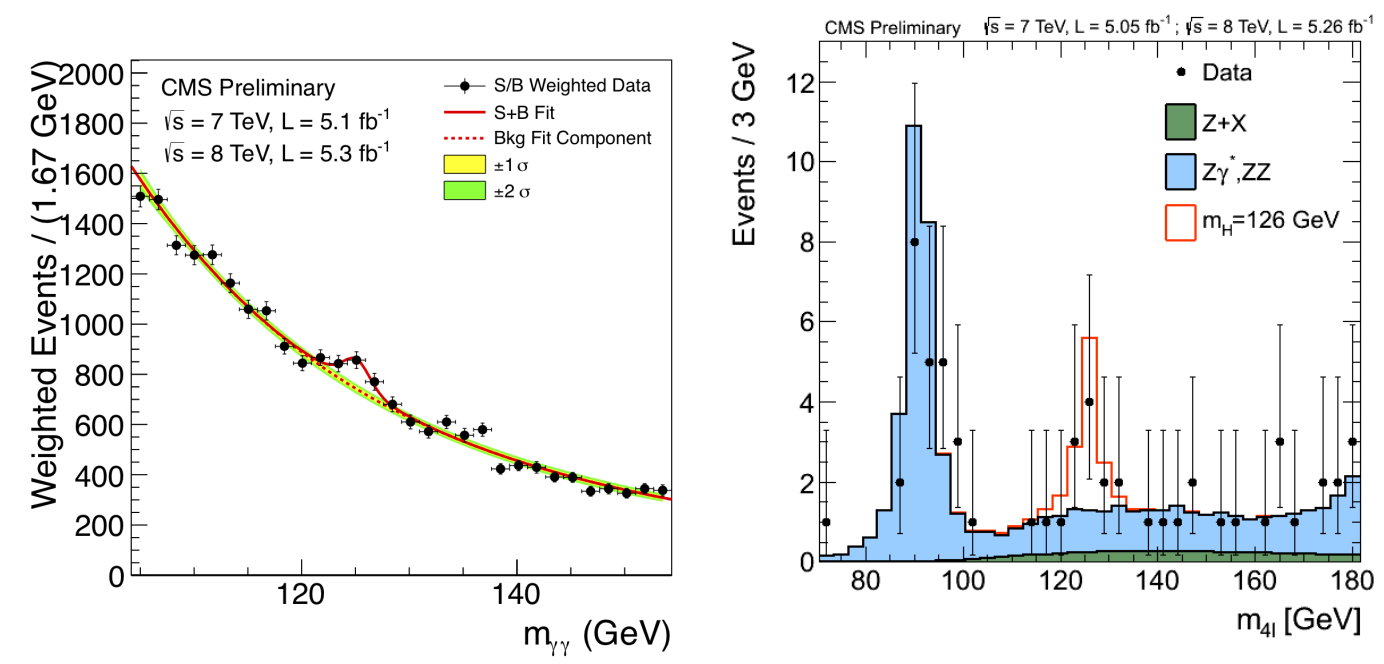

Figure 1.3: The invariant mass distributions for two decay modes for the Higgs boson, where the Higgs decays to two photons $(H \rightarrow \gamma \gamma)$ on the left and where the Higgs decays to two $Z^{0}$ bosons, which then decay into four leptons $(H \rightarrow Z Z \rightarrow l l l l)$ on the right. Both distributions show an excess of events at $\mathrm{m} \sim 125 \mathrm{GeV}$. Data collected during the 2011 and 2012 runs are used for both distributions. 


\subsection{The Parton Model and QCD}

Quantum Chromodynamics (QCD) is the study of interactions between quarks and gluons, which are collectively known as partons. The discovery that hadrons were made up of smaller constituents occurred in 1969, during a series of deep inelastic lepton-hadron scattering (DIS) experiments in which leptons collided with stationary hadrons.

Quantum Electrodynamics (QED) is a gauge theory that describes electromagnetic interactions and was the model used for the development of QCD. The strength of an electromagnetic interaction is determined by its coupling constant, $\alpha\left(Q^{2}\right)$, which after renormalization takes the form:

$$
\alpha\left(Q^{2}\right)=\frac{\alpha(\mu)}{1-\frac{\alpha\left(\mu^{2}\right)}{3 \pi} \log \left(\frac{Q^{2}}{\mu^{2}}\right)}
$$

where $\mu$ is the renormalization term and $Q^{2}$ represents the momentum transfer between an incoming parton and an outgoing parton. The expression shown in Eq. 1.1 describes how the coupling constant $\alpha\left(Q^{2}\right)$ increases with increasing $Q^{2}$, which represents the amount of momentum transferred between particles during an interaction, momentum transfer.

Similar to QED, the strength of QCD interactions are governed by its own coupling constant, $\alpha_{s}\left(Q^{2}\right)$, given by:

$$
\alpha_{s}\left(Q^{2}\right)=\frac{1}{b \ln \left(\frac{Q^{2}}{\Lambda_{Q C D}^{2}}\right)}
$$

where $b=\frac{13-2 f}{12 \pi}$ and $f$ is the number of quark flavors [9]. The variable $\Lambda_{Q C D}(\sim 200$ $\mathrm{MeV})$ is the parameter indicating the order of magnitude where $\alpha_{s}\left(Q^{2}\right)$ is large. 
Unlike the QED coupling constant $\alpha\left(Q^{2}\right)$, which increases with increasing $Q^{2}$, the QCD coupling constant decreases with increasing momentum transfer. High values of $Q^{2}$ occur at short distances from the interaction point $(<1 \mathrm{fm})$. At short ranges the strength of $\alpha_{s}\left(Q^{2}\right)$ is small enough that the partons confined within the hadron may be treated as individual 'free' particles, an effect known as asymptotic freedom. The distribution shown in Fig. 1.4 describes the behavior of $\alpha_{s}$ as a function of the energy scale [6]. It is the nature of $\alpha_{s}$, in which the strength at high $Q^{2}$ and short distances weakens, that allows for hadronic collisions at high energies to be treated as elastic collisions at the parton level.

As outgoing partons gain distance from the interaction point the strength of $\alpha_{s}\left(Q^{2}\right)$ increases. Outgoing partons begin producing quark-antiquark pairs and later combine with other partons to form hadrons, a process known as fragmentation or hadronization [29]. These hadrons are formed within close proximity to each other, creating a collimated set of hadrons known as jets. Jets are defined as a group of hadrons within a cone in $\eta-\phi$ phase space.

The distribution shown in Fig. 1.5 is a depiction of two partons colliding with each other. Notice that the remaining partons belonging to the hadrons do not collide with each other and thus are not part of the interaction. These remaining particles make up the underlying event (UE), which is the name given for objects that accompany the hard scattering event but have no active role in the collision. [30]. Minimum Bias, or Min-Bias, are inelastic collisions passing physics triggers that accompany the interaction. The Min-Bias cross section is $\sim 100 \mathrm{mb}$ and is an irreducible background to all physics analyses at the Large Hadron Collider.

The probability that parton $a$ is found within proton $A$ with a momentum fraction between $x$ and $x+d x$ is determined by parton distribution functions (PDF). These 


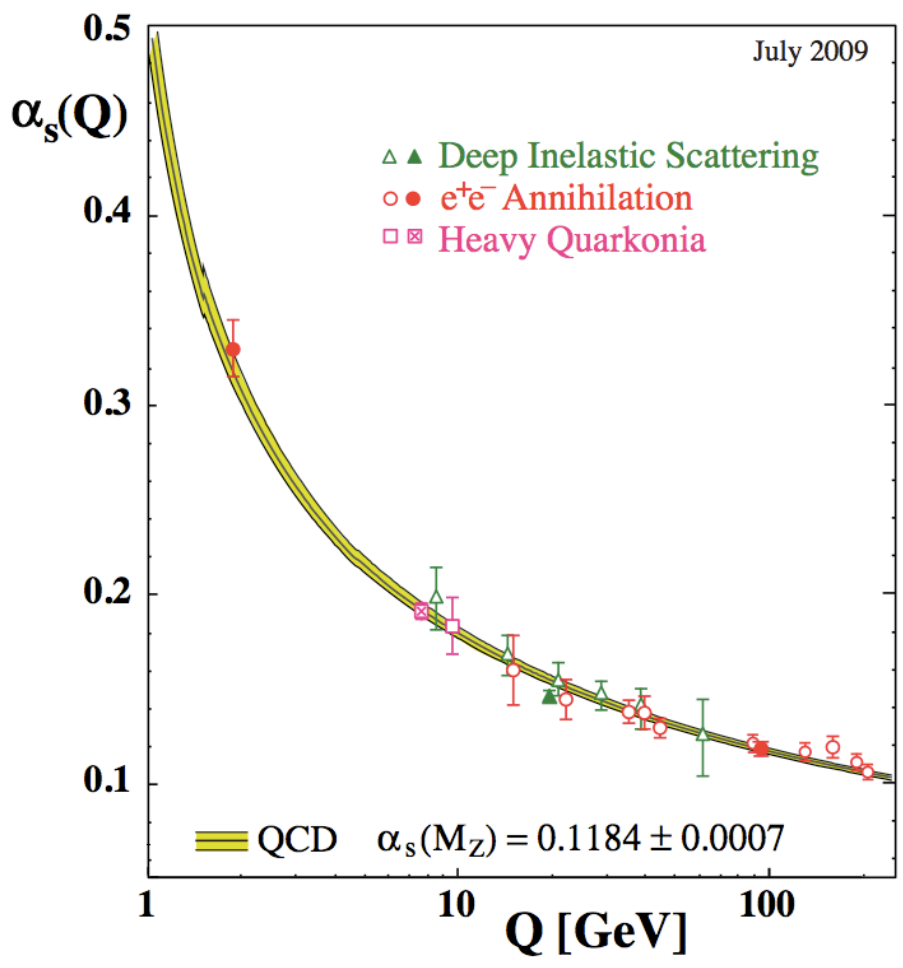

Figure 1.4: Measured values of $\alpha_{s}$ as a function of the energy scale $Q$ [6].

functions describe how a particle probing the proton would 'view' its structure. All partons within the proton have a PDF associated with it. Parton Distribution Functions are independent of the type of collisions in which the partons are involved and are universal parameters in QCD which are experimentally determined [22]. The distribution in Fig. 1.6 shows the PDFs for quarks and gluons using the NNLO MSTW2008 Parameterization [2].

We may write an expression for the cross section of the hadronic collision shown in Fig. 1.5 in terms of the PDFs and the partonic cross section:

$$
E \frac{d \sigma}{d p^{3}}=\frac{d \sigma}{d y_{b} d p^{* 2} d \cos \theta}=\frac{1}{\hat{s}} \sum \frac{G\left(x_{1}\right) G\left(x_{2}\right)}{x_{1} x_{2}} \frac{d \hat{\sigma}}{d \cos \theta^{*}}
$$




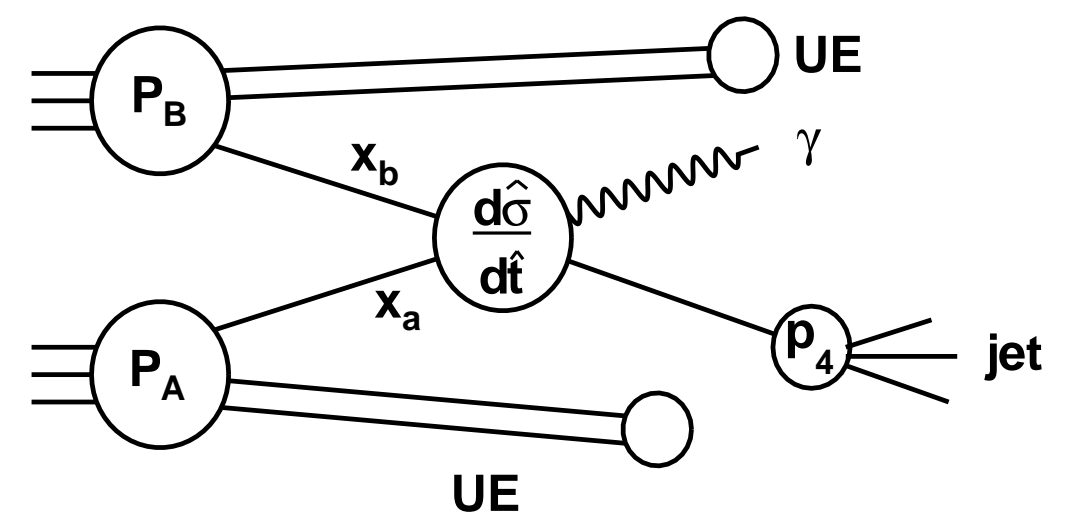

Figure 1.5: Schematic view of $p p$ collision. Parton $a$ from proton $A$ interacts with parton $b$ from proton $B$ to form the hard scatter. One of the outgoing partons fragments and forms a jet.

where $G\left(x_{i}\right)$ represents the PDF for the $i^{\text {th }}$ parton involved in the collision, $y_{b}$ is the boost in rapidity that may be used to transform between the lab and the center-ofmass frame and $p^{*}$ is the momentum of the outgoing particle in the center-of-mass (CM) frame. The CM energy, $\hat{s}$, is simply the square sum of the momentum 4-vector of $A$ and $B$ which represent the incoming hadrons. The fractional momentum of the incoming partons from protons $A$ and $B$ are represented by $x_{1}$ and $x_{1}$, respectively.

The expression shown in Eq. 1.3 must be calculable and give a finite result, otherwise experimental results can not be compared with theory. Quantum Chromodynamics makes use of renormalization techniques in which additional terms are included to handle the following divergences encountered in the theory: ultraviolet (UV), infrared (IR) and collinear divergences. These divergences can cause incalculable results (i.e., infinities for cross section measurements), which fares poorly when trying to validate theories experimentally. If the cross section $\rightarrow \infty$ it is the result of a UV divergence, if a partons energy $\rightarrow 0$ it is known as an IR divergence and if the angle between the outgoing partons $\rightarrow 0$ it is known as a collinear divergence. These divergences are 

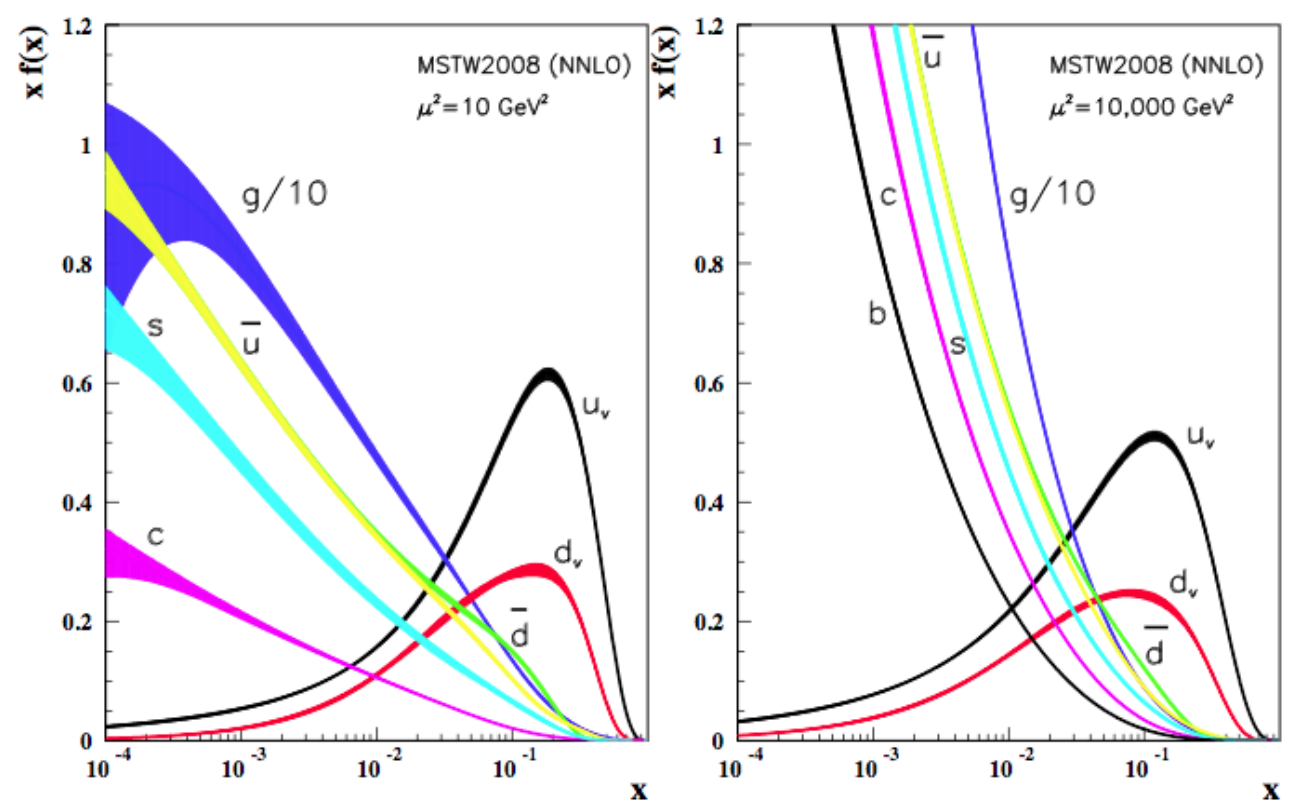

Figure 1.6: Parton distribution functions of quarks and gluons as a function of their fractional momentum, $x$, when the scale is $\mu^{2}=10 \mathrm{GeV}^{2}$ (left) and when the scale is $\mu^{2}=10,000$ $\mathrm{GeV}^{2}$ (right) [2].

handled with the introduction of scale factors. The renormalization scale, $\mu_{R}$, is traditionally taken to be of the same value as the analyzed outgoing particle's transverse momentum, $p_{T}$, and reflects the energy scale of the interaction. The factorization scale, $\mu_{F}$, assists in handling collinear divergences. The fragmentation scale, $\mu_{f}$, is analogous to $\mu_{R}$ and is used in fragmentation functions, which give the probability that a parton will produce a final state particle during the fragmentation process [31]. While there are many renormalization schemes, the most widely used in QCD theory is the modified minimal subtraction scheme $(\overline{M S})$ [29].

The partonic cross section is expressed in terms of the angular distribution, $\cos \theta^{*}$, and may also be expressed in terms of the mandelstam variables $\hat{t}$ and $\hat{s}$ :

$$
\frac{d \hat{\sigma}}{d \cos \theta^{*}}=\frac{1}{\hat{s}} \frac{d \hat{\sigma}}{d \hat{t}}
$$


$\theta^{*}$ represents the angle outgoing partons make with respect to the beam axis in the CM frame as shown in Fig. 1.7 visually.
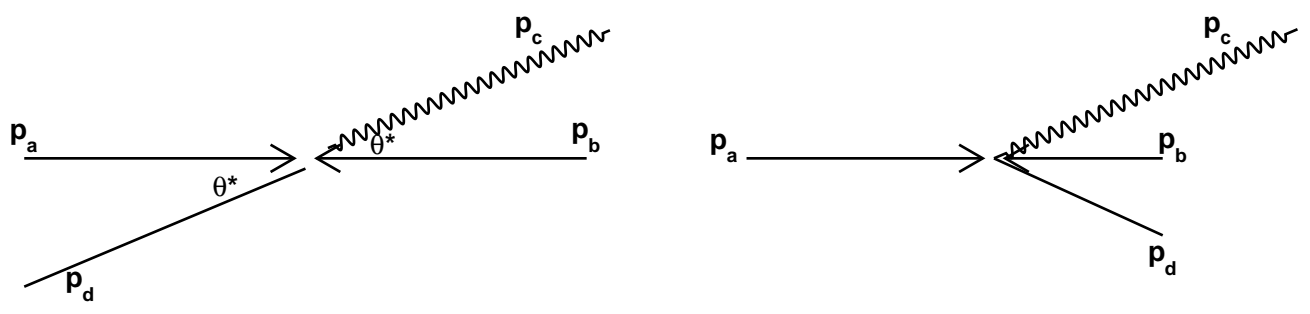

Figure 1.7: Collision in the CM frame (left) and in the boosted frame (right).

$$
\begin{gathered}
\hat{s}=\left(p_{a}+p_{b}\right)^{2}=4 \mathbf{p}^{2} \\
\hat{t}=\left(p_{a}-p_{c}\right)^{2}=-2 \mathbf{p}^{2}\left(1-\cos \theta^{*}\right) \\
\hat{u}=\left(p_{a}-p_{d}\right)^{2}=-2 \mathbf{p}^{2}\left(1+\cos \theta^{*}\right)
\end{gathered}
$$

The expressions in Eq. 1.5 show the direct relationship between the mandelstram variables and $\cos \theta^{*}$. The momenta in bold refers to the classical 3-vector momenta $(x, y, z)$ while the momenta italicized refers to the 4 -vector momenta $(E, x, y, z)$. The momentum of the partons is written as:

$$
p_{a}=\frac{x_{a} \sqrt{s}}{2} \text { and } p_{b}=\frac{x_{b} \sqrt{s}}{2}
$$

where $x_{a}$ and $x_{b}$ is the fractional momentum.

The $d \hat{\sigma} / d \cos \theta^{*}$ term is part of the prompt photon cross section shown in Eq. 1.3 , which can be measured as a standard test of next-to-leading order (NLO) QCD. Such measurements that set out to test NLO QCD, such as the prompt photon cross section measurement [32, 33, 34, 35, 36, 37] include comparing experimental results to Eq. 1.3 theoretical solution. The advantage of measuring the angular distribution of prompt photons is that the term involving $\cos \theta^{*}$, shown in Eq. 1.4, is indepen- 
dent of parton distribution functions, which contribute large uncertainties in such a measurement.

Measuring $\cos \theta^{*}$ requires boosting into the $\mathrm{CM}$ frame, which will limit the statistics available for this analysis. As an alternative, the angle $\eta^{*}$ is measured as an effective test of NLO QCD which is closely related to $\cos \theta^{*}$ :

$$
\begin{gathered}
\eta^{*}=\frac{\eta^{\gamma}-\eta^{j e t}}{2} \\
\cos \theta^{*}=\tanh \eta^{*}
\end{gathered}
$$

It is important to note that a test of NLO QCD of this kind has not been done in over a decade, since the early years of the Tevatron experiments at Fermi National Accelerator Laboratory (FNAL) as part of the DØ [7] and CDF [8] Collaborations, both measurements are shown in Figs. 1.8 and 1.9 , respectively.

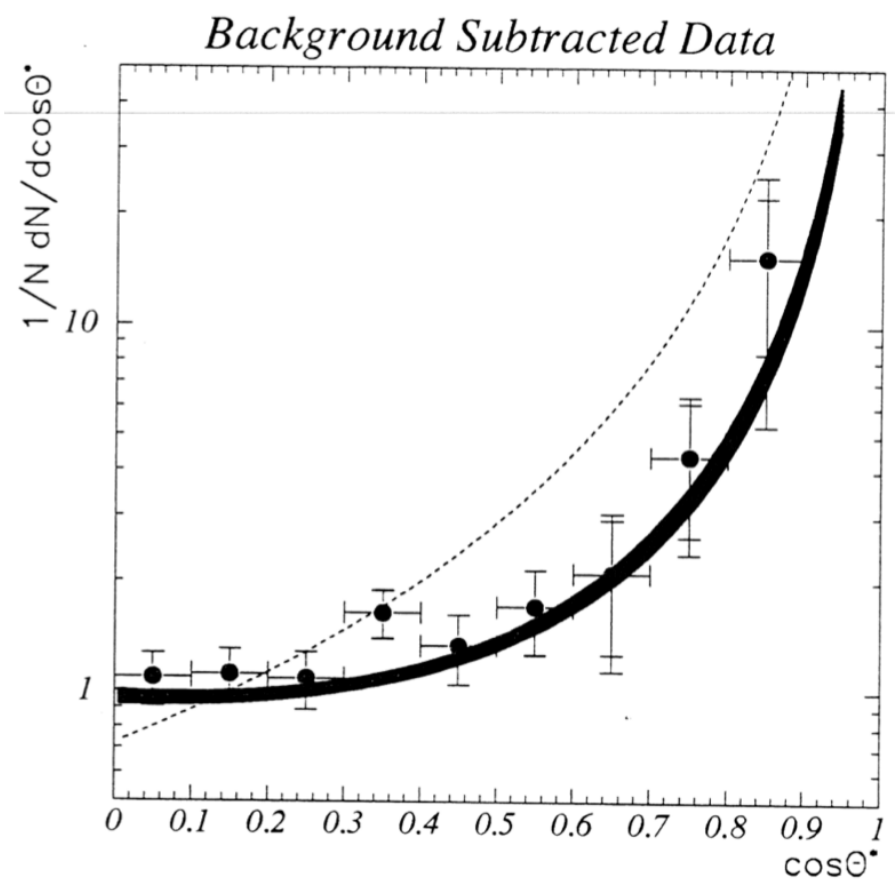

Figure 1.8: The $\left|\cos \theta^{*}\right|$ distributions from the $\mathrm{D} \varnothing$ collaboration [7]. The solid black line is the NLO prediction. The dotted line represents Rutherford scattering. The Data and theory agree. 


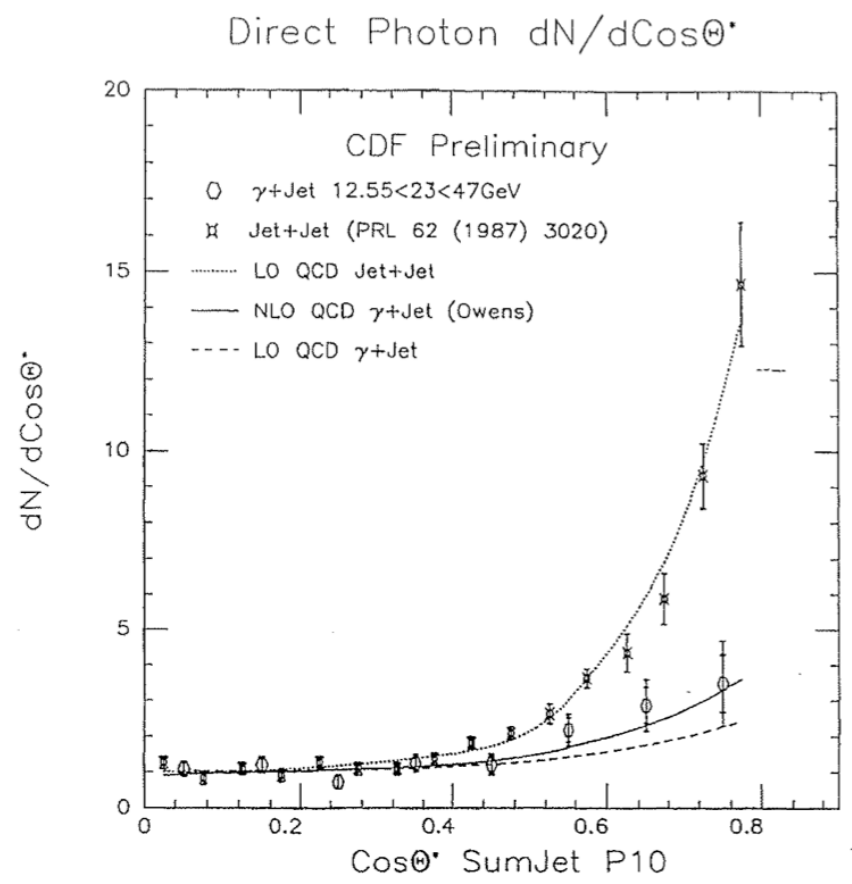

Figure 1.9: The $\left|\cos \theta^{*}\right|$ distribution from the CDF collaboration [8]. The open circles represent the angluar distribution in $\gamma+$ Jet events, which show good agreement with its NLO prediciton represented by the solid line. A factor of $\frac{1}{N}$ is missing in the y-axis labeling.

Prompt photons refer to any photons produced as a result of the hard scattering in $p p$ collisions. Prompt photons provide stringent testing of perturbative QCD (pQCD) and are a direct probe of the quarks and gluons within the colliding protons, giving further insight into the proton structure. Photons do not hadronize into other particles and therefore can be detected using electromagnetic calorimeters which tend to have a higher energy resolution than hadronic calorimeters.

\subsubsection{Leading Order Calculation}

The two dominant modes of prompt photon production are quark-anti-quark annihilation $(q \bar{q} \rightarrow g \gamma)$ and the QCD equivalent of Compton scattering $(q g \rightarrow q \gamma)$, in which 
a gluon strikes a quark resulting in a photon and a scattered quark; Fig. 1.10 shows the leading order (LO) Feynman diagrams.

In addition to giving a pictorial representation of a process, Feynman diagrams provide a recipe to calculate a processes amplitude, $|\mathcal{M}|^{2}$, and thus its cross section. One of the Feynman rules states that each vertex where a quark and/or gluon is present contributes a factor of $\alpha \alpha_{s}$ to the amplitude [23]. Fragmentation photons $(g g \rightarrow g g)$ are included at LO since its vertex evolves as $\frac{\alpha}{\alpha_{s}}$, thus when $|\mathcal{M}|^{2}$ is calculated it is of the order $\alpha \alpha_{s}$.

Table 1.3: Partonic differential cross sections for prompt photon production at leading order [3]. The dependencies on $\pi, \alpha_{s}, \alpha$ and $\hat{s}$ have been removed for simplicity.

\begin{tabular}{|c|c|}
\hline Process & $|\mathcal{M}|^{2}$ \\
\hline$q+\bar{q} \rightarrow \gamma+g$ & $\frac{8}{9}\left[\frac{\hat{u}}{\hat{t}}+\frac{\hat{t}}{\hat{u}}\right]$ \\
$g+q \rightarrow \gamma+q$ & $-\frac{1}{3}\left[\frac{\hat{u}}{\hat{s}}+\frac{\hat{s}}{\hat{u}}\right]$ \\
$g+g \rightarrow g+g$ & $\frac{9}{2}\left[3-\frac{\hat{t} \hat{u}}{\hat{s}^{2}}-\frac{\hat{s} \hat{u}}{\hat{t}^{2}}-\frac{\hat{s} \hat{t}}{\hat{u}^{2}}\right]$ \\
\hline
\end{tabular}

\subsubsection{Next to Leading Order Calculation}

Feynman diagrams for next-to-leading order (NLO) prompt photon production, which include final states with three outgoing particles shown in Figs. 1.11 and 1.12 for the $q g \rightarrow g \gamma$ and $q \stackrel{-}{\rightarrow} g \gamma$ processes, respectively. These higher order terms are a result of an incoming or an outgoing parton radiating a gluon during the process. The Feynman rules indicate the diagrams shown in Figs. 1.11 and 1.12 are of the order $\alpha \alpha_{s}^{2}$, which contribute to NLO calculations. 'One-loop' diagrams as shown in Fig. 1.13 are also included at NLO since the virtual gluon present in these diagrams contributes a factor of $\alpha \alpha_{s}^{2}$ to the cross section.

Singularities introduced by NLO diagrams are dealt with by introducing cut-off parameters that limit the phase space region to where the singularities are present. Soft singularities arise in the 3-body final state diagrams when the ratio between the radi- 

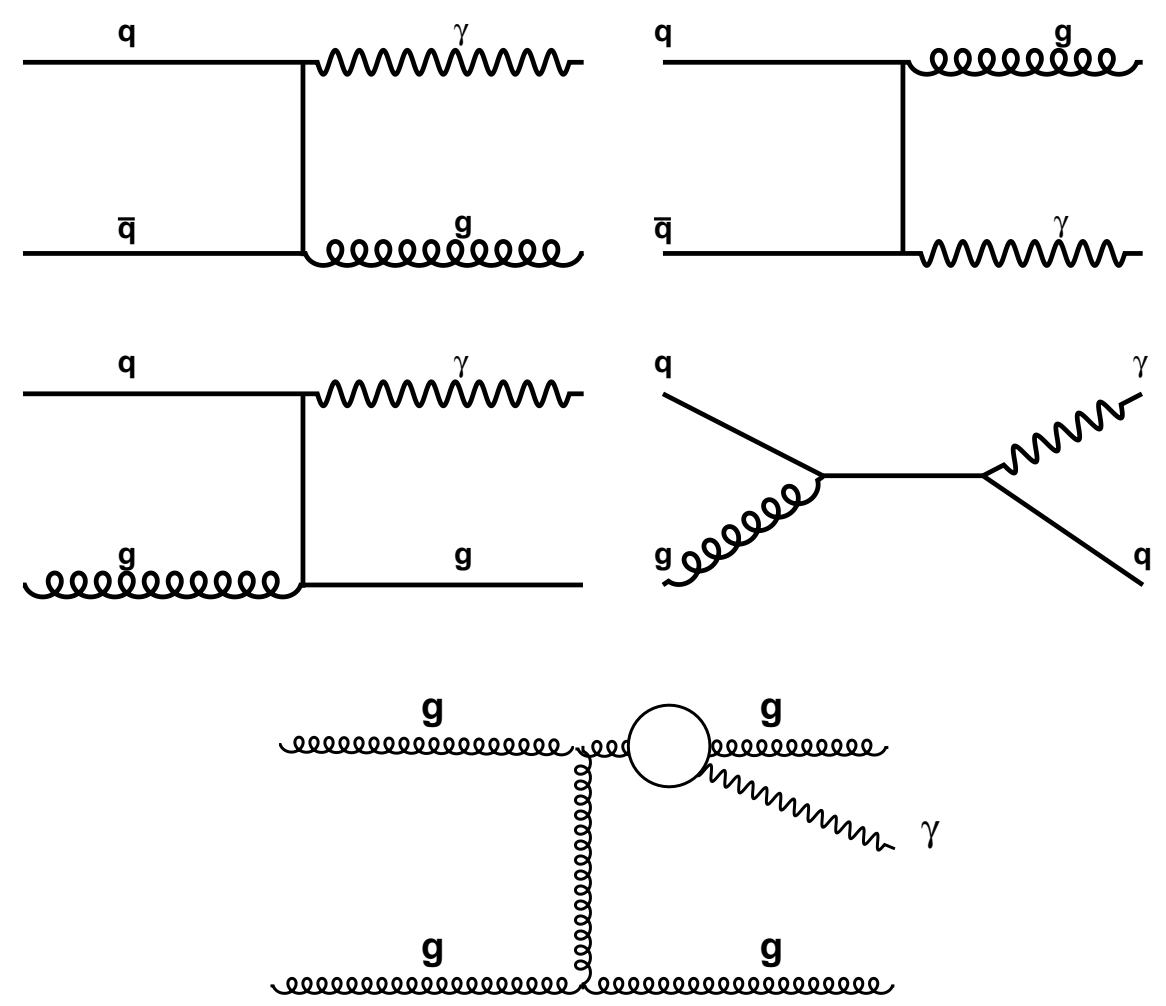

Figure 1.10: Leading order feynman diagrams for prompt photon production. Top diagrams show the $q \rightarrow g \gamma$ feynman diagrams at leading order. Middle diagrams shows the $q g \rightarrow g \gamma$ process for the t-channel(left) and the s-channel (right) at leading order. The feynman diagram for fragmentation photon production at leading order is shown at the bottom. The vertex in which the fragmented photon is present contributes a factor of $\frac{\alpha}{\alpha_{s}}$, making the process of the order $\alpha \alpha_{s}$.

ated gluons energy, $E_{g}$, and the CM energy approaches zero. The cut-off parameter $\delta_{s}$ separates the region of phase space where soft gluons arise, which is defined to be where $E_{g}$ in the rest frame of the interaction is less than $\frac{\delta_{s} \sqrt{s}}{2}$ in value. Collinear singularities are handled by the cut-off parameter $\delta_{c}$, which separates the region of phase space when Lorentz scalars $s_{i j}$ and $t_{i j}$, defined as:

$$
\begin{aligned}
s_{i j} & =\left(p_{i}+p_{j}\right)^{2} \\
t_{i j} & =\left(p_{i}-p_{j}\right)^{2}
\end{aligned}
$$


are less than $\delta_{c} s_{12}$ in value. Both $\delta_{s}$ and $\delta_{c}$ are selected such that they are sufficiently small enough to return finite results for both sets of NLO diagrams. Any singularities introduced have been removed and what is left are two sets of contributions to the NLO calculation that depend on their cut-off parameters, $\delta_{s}$ and $\delta_{c}$. These dependencies cancel when both contributions are combined, showing that the NLO calculation is independent of any scaling factors introduced during the regularization process. The distributions in Fig. 1.14 show the single jet cross section as a function of each cut-off parameter separately for each NLO contribution and then combined [10]. The steadiness in the cross section with increasing cut-off parameter values indicate that the NLO calculation is independent of any parameters that are introduced in order to obtain a finite result. It is this independence of the scale at NLO that increases the precision of the calculation compared to LO calculations. 

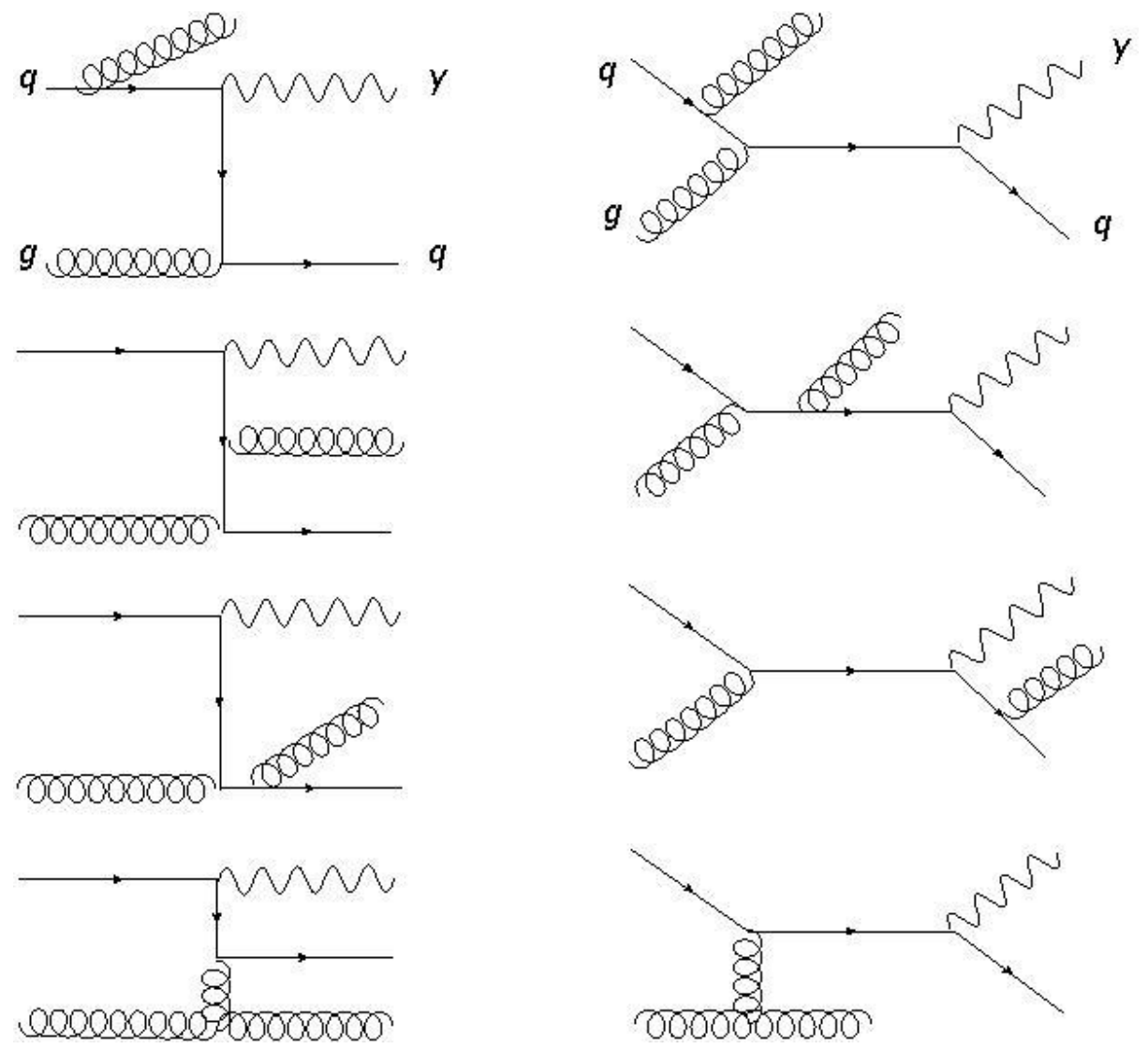

Figure 1.11: NLO Feynman diagrams for QCD Compton scattering. Adpated from [9]. 


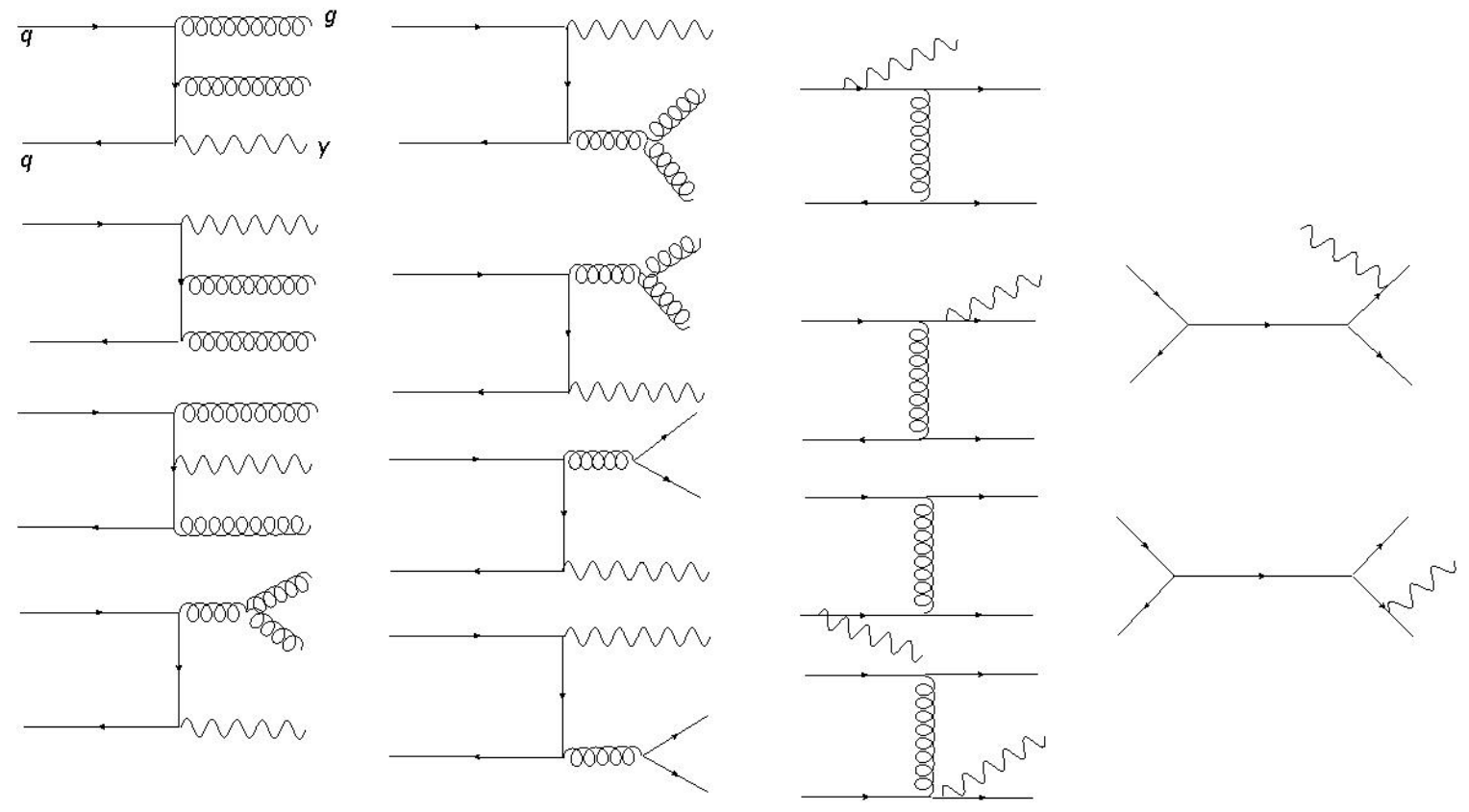

Figure 1.12: NLO Feynman diagrams for quark annihilation. Adapted from [9]. 

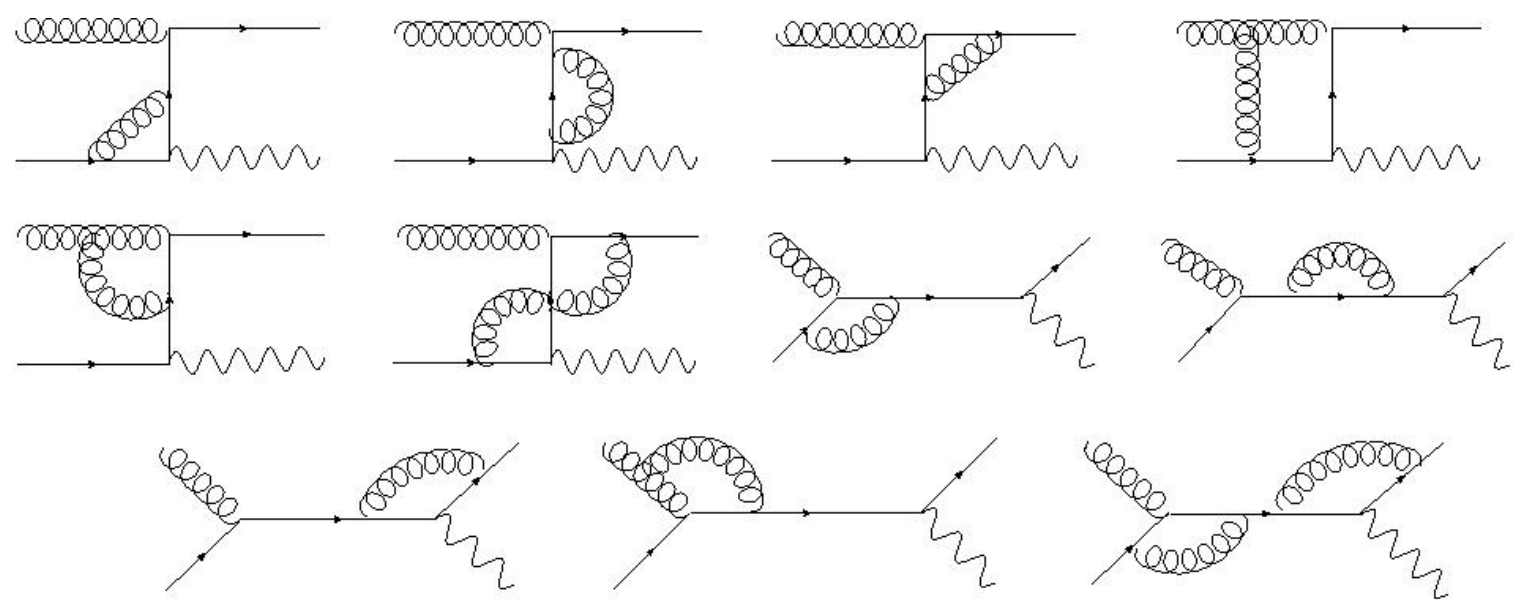

Figure 1.13: One-loop Feynman diagrams for prompt photon production. Adapted from [9] 

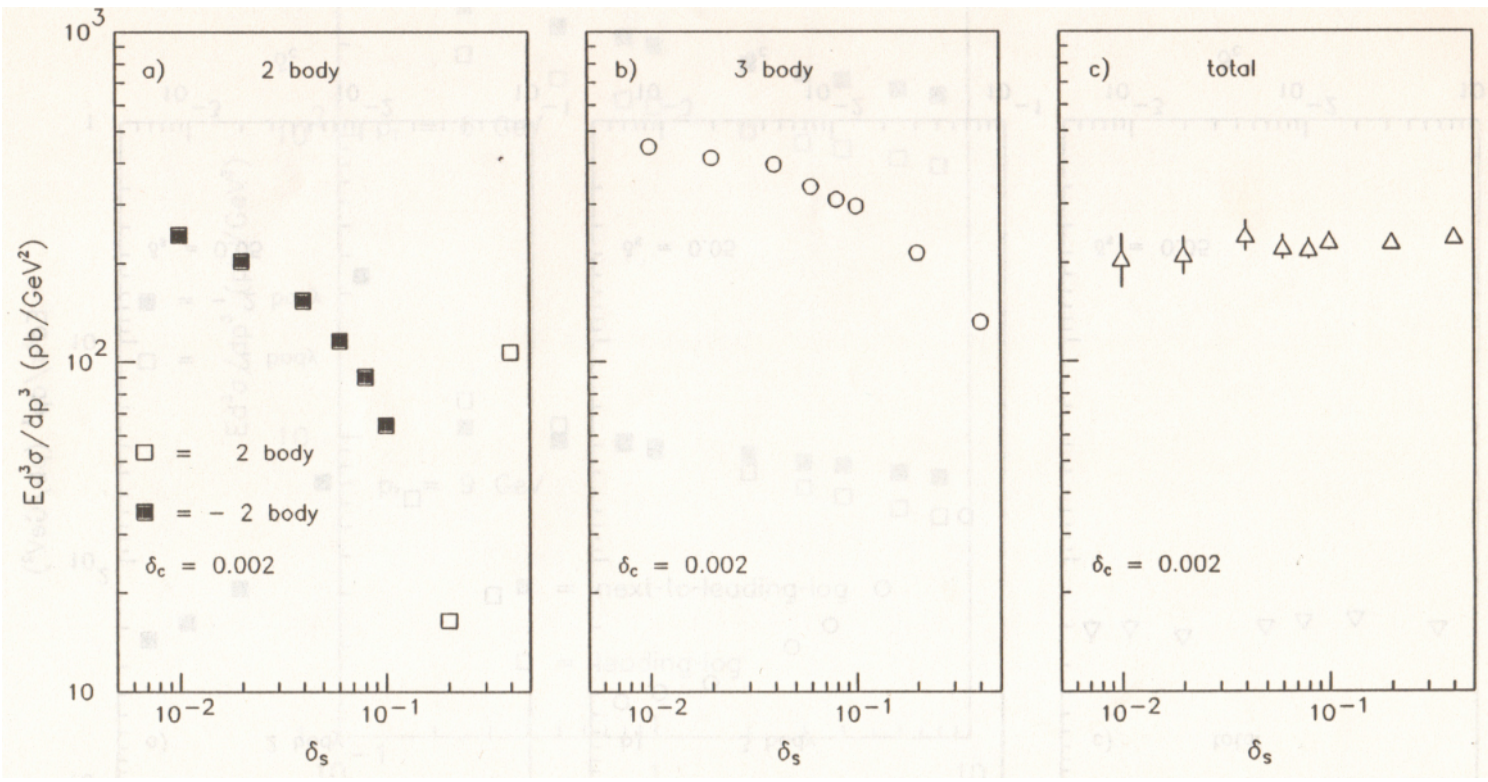

Figure 1.14: Single jet invariant cross section as a function of the cut-off parameter $\delta_{s}$ while keeping $\delta_{c}$ constant. The one-loop contribution is shown on the left, the 3-body final state contribution is shown on the middle and the combined result is shown on the right [10]. 


\section{CHAPTER 2}

\section{THE COMPACT MUON SOLENOID DETECTOR}

\subsection{The Large Hadron Collider}

The Large Hadron Collider (LHC) is a proton-proton $(p p)$ accelerator located at the European Organization for Nuclear Research (CERN) just outside of Geneva, Switzerland. The accelerator ring measures $26.7 \mathrm{~km}$ (17 mi) in circumference and is $100 \mathrm{~m}$ (roughly $300 \mathrm{ft}$ ) underground between the countries of Switzerland and France. The LHC collided protons at a center-of-mass (CM) energy of $\sqrt{S}=7 \mathrm{TeV}$ for the 2011 run year. In 2012, the LHC is currently colliding protons at a CM energy of $\sqrt{S}$ $=8 \mathrm{TeV}$.

The goal of the LHC experiments is to gain further knowledge of partonic substructure and investigate the existence of new physics phenomena. To reaffirm that future discoveries are in fact new physics, the LHC experiments will measure various known parameters included in the Standard Model. The anticipated discoveries not only require highly energetic collisions but also highly intense beams which require more interactions. Using the expression for the rate of interactions:

$$
\frac{d N}{d t}=\mathcal{L} \sigma
$$

where $\mathcal{L}$ is the instantaneous luminosity and $\sigma$ is the cross section, shows that in order to acheive the intense beams needed to investigate new physics a high value of $\mathcal{L}$ is needed. The expression for $\mathcal{L}$ is:

$$
\mathcal{L}=\frac{\gamma f k_{B} N_{p}^{2}}{4 \pi \sigma_{x} \sigma_{y}}
$$


where $\gamma$ is the Lorentz factor (7461), $f$ is the revolution frequency, $k_{B}$ is the number of proton bunches, and $N_{p}$ is the number of protons per bunch. The beam width in the $x$ and $y$ directions, noted as $\sigma_{x}$ and $\sigma_{y}$ respectively, were measured using Van Der Meer scans [38]. Van Der Meer scans measure the interaction area by calculating the interaction rate within each beam, which is done by using Fast Beam Current Transformers (FBCTs) that measure the beams current [39]. This luminosity measuring technique initially gave an uncertainty on the luminosity measurement of $11 \%$, but was later refined in order to give an uncertainty of $4 \%$. Relative uncertainty is measured using the Hadronic Forward Calorimeter and hits in the pixel tracker (discussed in the next section) which allows integration to obtain total luminosity per lumi-section.

The LHC is designed to have a peak luminosity of $10^{34} \mathrm{~cm}^{-2} \mathrm{~s}^{-1}$, with each proton beam having 2808 bunches $\left(k_{B}\right)$ separated by $25 \mathrm{~ns}$, with $1.15 \times 10^{11}$ protons per bunch $\left(N_{p}\right)$. The peak luminosity reached for the 2011 run year was $10^{33} \mathrm{~cm}^{-2} \mathrm{~s}^{-1}$.

A bottle of hydrogen gas is the source of the proton beams. Hydrogen atoms are stripped of its electron in order to produce a proton. These protons are then shot through the linear accelerator, Linac2, with an energy of $50 \mathrm{MeV}$ into the Proton Synchrotron Booster (PSB) ring (radius $25 \mathrm{~m}$ ) where they are accelerated until they have reached an energy of $1.4 \mathrm{GeV}$. From the PSB they are then injected into the Proton Synchrotron (PS) ring where protons are further accelerated to $26 \mathrm{GeV}$ and are formed into bunches of protons, spaced out $50 \mathrm{~ns}$ apart (25 ns more than the original design). From the PS ring the protons are then injected into the Super Proton Synchrotron (SPS) ring where proton bunches are accelerated to $450 \mathrm{GeV}$. Once the proton bunches have reached $450 \mathrm{GeV}$ they are injected into the LHC ring where they will collide once they have each reached an energy $3.5 \mathrm{TeV}$. The picture in Fig. 2.1 shows the rings locations relative to the LHC ring. The protons are directed along 


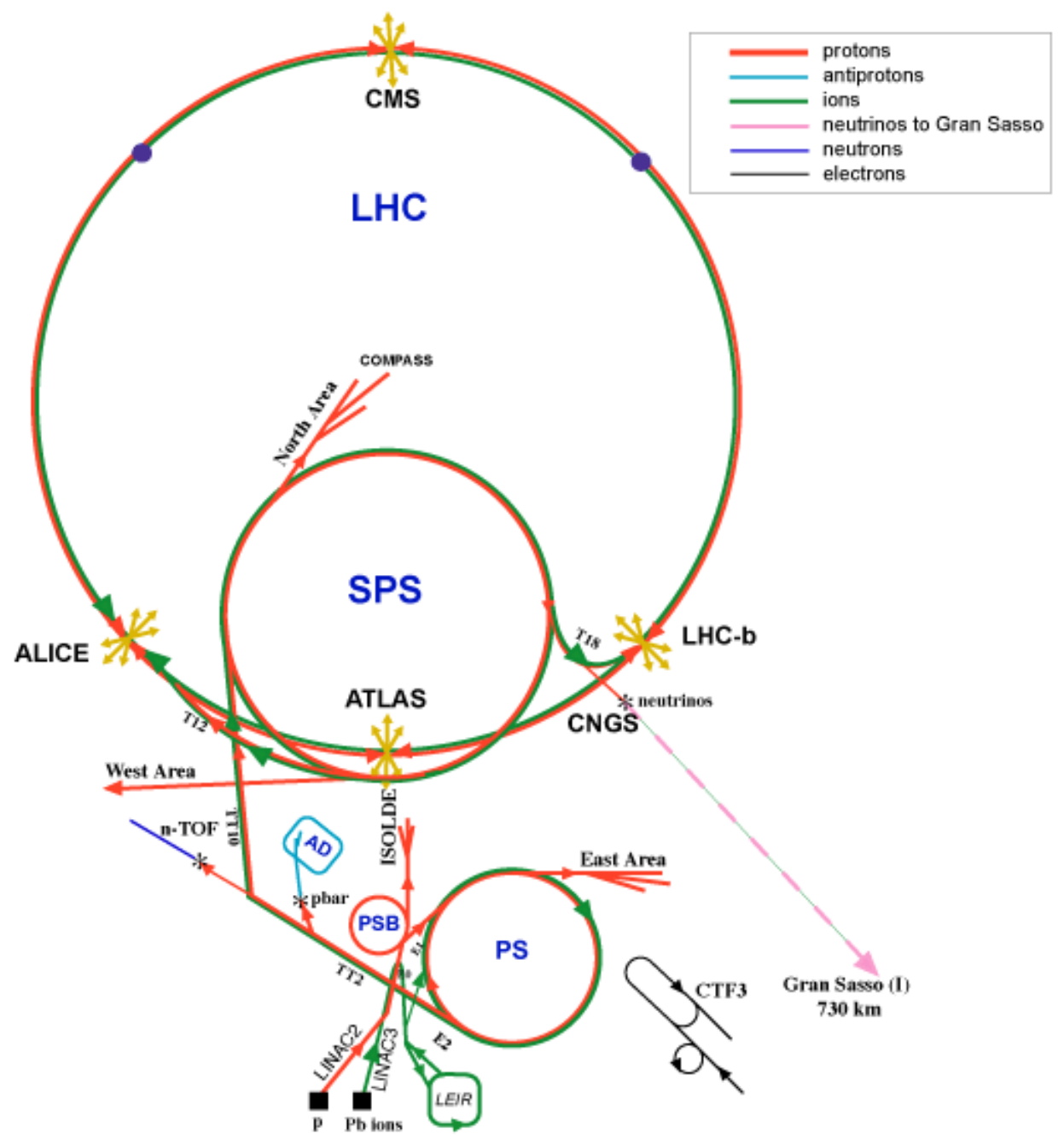

Figure 2.1: Pictorial representation of CERN's accelerator complex, the LHC ring and the experimental detectors [1].

the LHC ring circumference by a series of dipole magnets (1232 in total). The dipole magnets generate a 8.33 Tesla magnetic field. The dipoles were constructed using coils of niobium-titanium superconducting cables and are cooled to a temperature of $1.9 \mathrm{~K}$ using liquid helium [40].

Though the LHC was initially designed to run at a $\sqrt{S}=14 \mathrm{TeV}$, it has only reached a little over half that value. First collisions at the LHC ring were observed on September 9, 2008. Ten days later a faulty electrical connection between the dipole magnets 
resulted in a large helium leak in Sector 3-4 within the LHC ring. The leak occurred when the accelerator department was testing the LHC machine to see how it would react to running at $5 \mathrm{TeV}$. Extreme measures were taken by the CERN staff to ensure an incident like this would not occur again during runtime, and two years later the LHC was back in commission and running first at $\sqrt{S}=7 \mathrm{TeV}$ and now at $8 \mathrm{TeV}$, still making it the highest energetic hadronic accelerator in the world.

\subsection{The Compact Muon Solenoid Detector}

The Compact Muon Solenoid (CMS) detector is one of two general purpose detectors built to study physics at the Large Hadron Collider. It is located about $100 \mathrm{~m}$ underground near the French town of Cessy, located between Lake Geneva and the Jura mountains. The detectors location in Fig. 2.1 shows its location is across the ring from the competing experiment, ATLAS. The detector is $21.6 \mathrm{~m}$ long with a diameter of $14.6 \mathrm{~m}$ and weights 12,500 tons. Installation of the detector was completed in 2008 and saw the first successful collisions in March 2010. The experiment name gives a general description of the detector:

- Compact: Its size is small when compared to the second general purpose detector at the LHC, A Toridal LHC ApparatuS (ATLAS)

- Muon: The detector has been optimized to detect muons from $p p$ collisions

- Solenoid: Refers to the type of magnet used to generate a magnetic field

The detector uses a right-handed coordinate system. The origin is centered at the interaction point (IP) within the detector, with the y-axis pointing vertically upward and the $\mathrm{x}$-axis pointing radially inward towards the center of the LHC ring. The z-axis points along the beam direction towards the Jura mountains. The azimuthal angle $\phi$ is the angle measured from the $\mathrm{x}$-axis in the $\mathrm{x}-\mathrm{y}$ plane. The polar angle $\theta$ is 


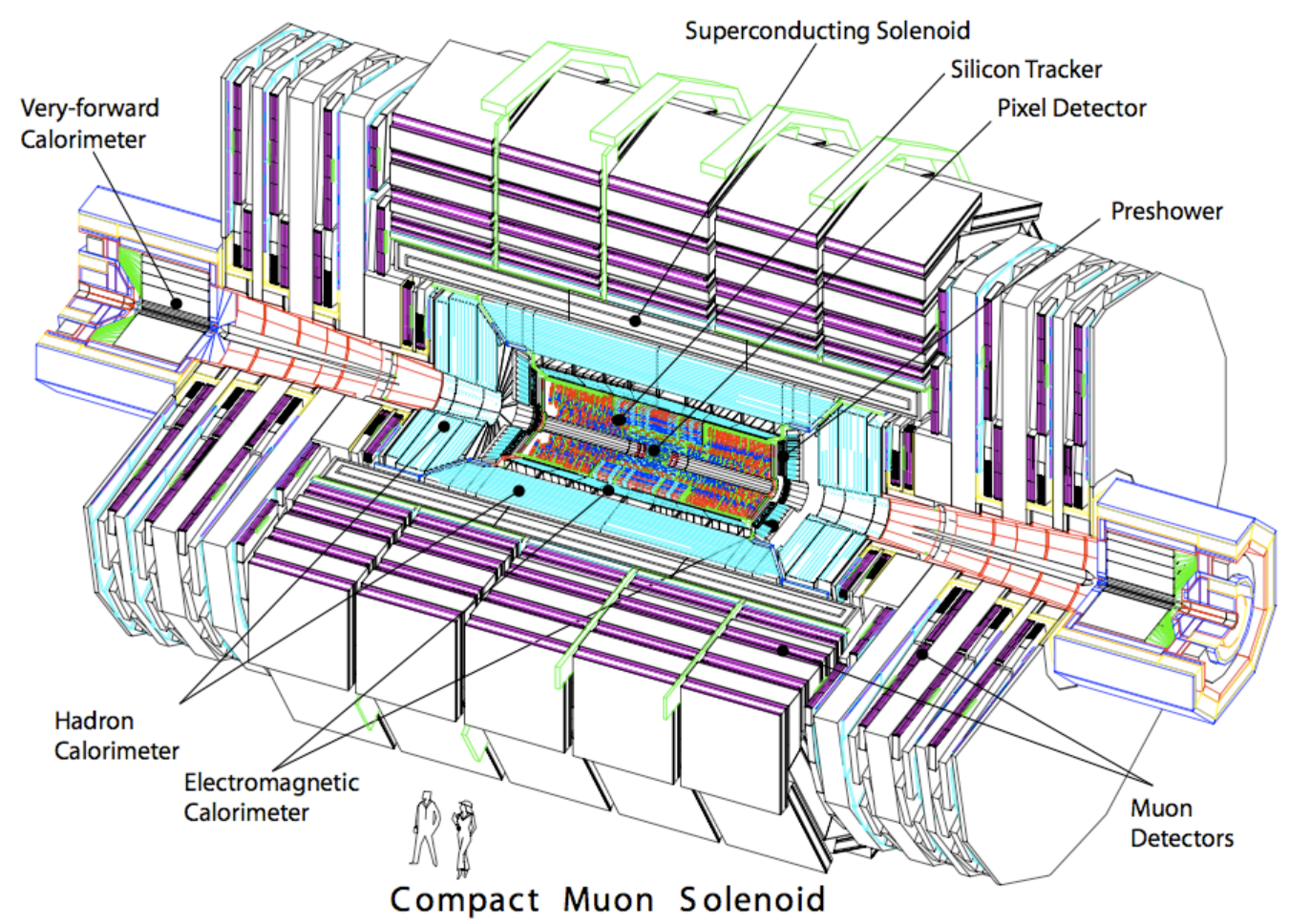

Figure 2.2: The Compact Muon Solenoid Detector [4].

measured from the z-axis. The pseudorapidity is defined as:

$$
\eta=-\ln \tan \frac{\theta}{2}
$$

The pseudorapidity is the same as the rapidity, $Y$, for massless particles ${ }^{1}$.

The momentum and energy measured transverse to the beam direction are computed from the $\mathrm{x}$ and y components and are denoted as $p_{T}$ and $E_{T}$, respectively.

The CMS detector is comprised of four main sub-detectors, each of which aids in meeting the goals of the LHC physics program:

- The Tracking System: High in resolution of charged particle momentum. The pixel detectors near the IP allow for high efficiency of triggering as well as

$$
{ }^{1} Y=\frac{1}{2} \ln \left(\frac{E+p_{z}}{E-p_{z}}\right)
$$


offline tagging of $\tau$ and $b$-jets which assists in analysis searching for beyond the Standard Model physics analyses.

- The Electromagnetic Calorimeter (ECAL): The lead tungstate scintillating crystals make this calorimeter compact with high granularity ideal for the discovery of a light Higgs boson that decays to two photons [41].

- The Hadronic Calorimeter (HCAL): Encapsulates ECAL and is made of brass alloy absorber plates with scintillator tiles between each plate. Hadronic particles traverse HCAL and interact with the absorber plates, causing a cascade of particle showers. It lies within the confines of the superconducting solenoid magnet.

- The Muon System: Located on the outer most part of the detector, it identifies muons and measures their momentum with the help of the magnetic field produced by the superconducting solenoid magnet.

With the exception of the muon system, the rest of the sub-detectors are encapsulated within the $13 \mathrm{~m}$ long, $5.9 \mathrm{~m}$ in diameter superconducting solenoid magnet.

\subsubsection{Magnet}

One of the goals CMS set out to accomplish was to design and build a muon system that could detect muons better than any other collider experiment, which required a system with a momentum resolution of $10 \%$ for a muon with $p=1 \mathrm{TeV}$ [4]. The CMS magnet is a large $(12.9 \mathrm{~m}$ in length) superconducting solenoid with a $3.8 \mathrm{~T}$ magnetic field, which will give particles larger bending power as they traverse the detector. The combination of a large magnet and high magnetic field ensures a high momentum resolution, especially in the forward region of the detector. Table 2.1 shows specifications of the magnet. 
Table 2.1: Parameters of the CMS Solenoid Magnet

\begin{tabular}{|c|c|}
\hline Field & $3.8 \mathrm{~T}$ \\
\hline Inner Bore & $5.9 \mathrm{~m}$ \\
\hline Length & $12.9 \mathrm{~m}$ \\
\hline Number of Turns & 2168 \\
\hline Current & $19.5 \mathrm{kA}$ \\
\hline Stored Energy & 2.7 GJ \\
\hline
\end{tabular}

\subsubsection{The Tracking System}

The tracking system is an integral part in the identification and study of charged particles. It is also the closest of all sub-detectors to the interaction point. Charged particles bend when traversing the tracking system due to the magnetic field applied by the superconducting solenoid magnet. The picture in Fig. 2.3 shows a quarter view of the tracker. The tracking system is made up of silicon hybrid pixel detectors and microstrip sensors and has a total coverage up to $|\eta|<2.4$ which includes the central and forward regions of the detector. The outer radius of the CMS tracker is nearly $110 \mathrm{~cm}$ and is $540 \mathrm{~cm}$ in length.

The silicon is prepared for detecting charged particles that traverse the tracking system by first introducing impurities into the silicon detector (a process known as doping) to change its conductivity. An impurity atom such as phosphorus, which has five outer electrons, is introduced and all but one of the electrons form bonds with its neighboring atoms. Silicon has four outer electrons, all of which bond to its neighboring atom in the lattice structure. The doping process leaves a (nearly) free electron and is known as $n$-type doping since there is an access of negative charge. Once the n-type doping is done, impurity atoms with three outer electrons, such as boron, are applied to the surface of the silicon in a process known as $p-t y p e$ which indicates an excess of positive charge. When this impurity atom is introduced into the lattice structure, it 'steals' an electron from somewhere else in the silicon structure 
in order to bond to its neighboring atoms. Joining the n-type and p-type creates a diode in which current is allowed run through the silicon. When $\mathrm{n}$ and p-types are combined it forms a $p-n$ junction in which there is the p-type area, the n-type area and a depletion area in between. No current runs through the material since the extra electrons that were left behind by the phosphorus fills in the holes left by the boron. The depletion area expands when a voltage is applied to the diode allowing maximum detection of a charged particle traversing the silicon. Once the charged particle traverses the silicon detector, it creates a current that is read out [42, 43].

The momentum of charged particles is measured using their position recorded by the tracking system and the strength of the magnetic field. Charged particles follow a curved trajectory as they traverse a magnetic field; the radius of curvature, $r$, of that trajectory along with the magnetic field strength $(B)$ is used to calculate their momentum [2]:

$$
p=0.3 * B * r
$$

The two types of silicon detectors that comprise the tracking system; the microstrip and hybrid pixel detectors are discussed.

\section{Microstrip Tracker}

The microstrips in the barrel (central) region of the tracking system is broken up into

two regions: the Tracker Inner Barrel (TIB) and the Tracker Outer Barel (TOB). The TIB consist of four layers and extends to $|z|<65 \mathrm{~cm}$. The strip sensors are $320 \mu \mathrm{m}$ in thickness and have a pitch that varies from $80-120 \mu \mathrm{m}$. The first two layers of the TIB consists of 'stereo' modules angled at $100 \mathrm{mrad}$ in order to provide measurements in both $r-\phi$ and $r-z$ coordinates, resulting in single-point resolution 


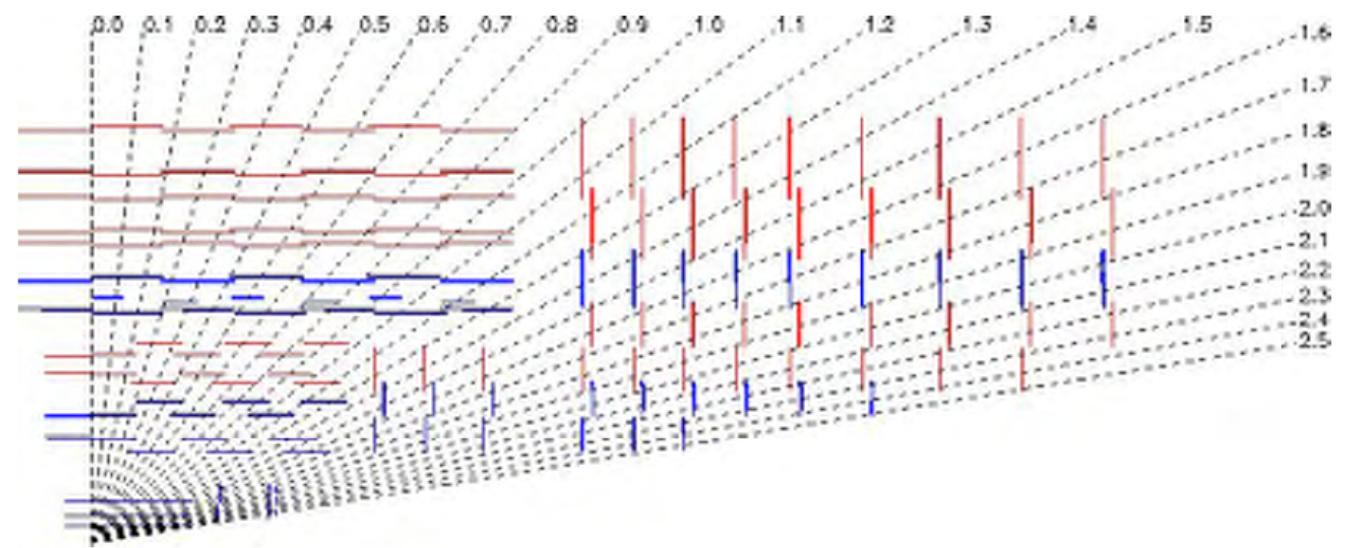

Figure 2.3: Quarter view of the CMS tracking system [4]. The tracking system consists of a combination of microstrip silicon detectors and pixel silicon detectors in the barrel (horizontal lines) and the endcap regions (vertical lines).

between 23-34 $\mu \mathrm{m}$ in $r-\phi$ and $230 \mu \mathrm{m}$ along the $z$-axis. The TOB consists of 6 layers and extends further in $z$ than the TIB, up to $|z|<100 \mathrm{~cm}$. The extended length of TOB is to avoid excessively shallow track crossing angles. The strip sensors in the TOB are also thicker $(500 \mu \mathrm{m})$ and can have higher pitches $(120-180 \mu \mathrm{m})$ than those present in the TIB. The first two layers of the TOB also consist of stereo modules angled at $100 \mathrm{mrad}$ intended for measurements in both $r-\phi$ and $r-z$ coordinates. The single-point resolution in this region varies from 35-52 $\mu \mathrm{m}$ in $r-\phi$ and $530 \mu \mathrm{m}$ along the $z$-axis.

The strip tracker endcaps are also divided into two regions: the Tracker Endcap (TEC) and the Tracker Inner Disks (TID). The TEC consists of nine disks and are located $120<|z|<280 \mathrm{~cm}$. The strip sensors located in the three innermost layers of the TEC have a thickness of $320 \mu \mathrm{m}$, while the rest of the strips are $500 \mu \mathrm{m}$. The TID consists of three disks, with all its strips $320 \mu \mathrm{m}$ thick and fills the gap between the TIB in the barrel and the TEC in the endcap. These disks are shown in Fig. 2.3 as the three vertical lines to the right of the Tracker Inner Barrel. Arranged in rings and centered on the beam line, the TEC and TID have strips pointing towards the beam 
line. The first two rings of the TID have stereo modules while the two innermost rings and the fifth ring of the TEC has the stereo modules. The strip detector in its entirety has 15,400 modules mounted on carbon fiber structures and is housed inside a temperature controlled outer support tube. The strip tracker operates at a temperature of $-20^{\circ} \mathrm{C}$.

The readout system for the strip tracker consists of a APV25 readout chip, analog optical links and a Front-End Driver (FED) processing board. The role of the readout chip is to sample, amplify, buffer, and process signals recorded from 128 channels of a strip sensor. The strip is then read out by an amplifier that is sensitive to charge. The output voltage from the amplifier is then sampled and stored to be read out by the triggering system at CMS. A more detailed description of the strip tracker readout system can be found in [44].

\section{Pixel Tracker}

The pixel barrel is made up of three layers of hybrid pixel detectors, with each layer divided into two half-cylinders each of which are $53 \mathrm{~cm}$ in length. One layer of pixel detectors are located at radii 4.4, 7.3 and $10.2 \mathrm{~cm}$. The pixels nearly square-shaped design $\left(100 \times 150 \mu \mathrm{m}^{2}\right)$ in $(r, \phi)$ and $z$ was implemented in order to achieve optimal vertex position resolution. The pixel barrel in total has 768 pixels arranged in halfladders of four identical modules each.

The pixel endcap is made up of two disks located on each side at $|z|=34.5 \mathrm{~cm}$ and $46.5 \mathrm{~cm}$ and has a turbine-like geometry with seven modules acting as a blade. In total the endcap is composed of 672 pixels.

In the barrel, a pixel module is read out by 16 Read-Out Chips (ROCs). Read-Out Chips are also used in the endcap, but each ROC can read information of 2-10 pixel modules. The signals of each pixel and its location are stored in a data buffer while 
the triggering system decides whether the event goes on to the next stage of data processing.

\subsubsection{The Calorimeters at CMS}

The scintillators making up ECAL and HCAL absorb a particle's energy deposit and converts it to light. Photodetectors are used to convert the light deposition into either a voltage or current which is then transformed into a digitized format for analysis.

\section{The Electromagnetic Calorimeter}

The Electromagnetic Calorimeter sits between the tracking system and the hadronic calorimeter. The ECAL detects all electromagnetic particles traversing the CMS detector. The ECAL is integral to photon studies as it is the only sub-detector that can be used to identify them.

The ECAL is able to measure the energy of electrons and photons due to their interaction with the crystals that comprise the calorimeter. At relativistic energies, electrons radiate photons when they are either accelerated or decelerated, in a process known as bremstrahlung [2]. The radiated photon may interact with the material resulting in converting into an electron-positron pair. As more particles are generated, the energy they have decreases. The summed energy of the particle shower resulting from bremstrahlung or pair production reflects the initial energy of the electron or photon and is collected by ECAL to assign the energy of the initial particle.

The radiation length $\left(X_{0}\right)$ is the distance a particle travels before it loses $1 / e$ of its energy. Within ECAL it is the electron whose radiation length is of most interest. The longitudinal length of the material must be at least $20 X_{0}$ in length in order to fully absorb the shower. 
The lateral component of an electromagnetic shower begins fairly narrow, with the amount of less energetic particles that surround it increasing with increasing shower depth. About $90 \%$ of the lateral component of the shower is enclosed within a cylinder of radius equal to one Moliére radius, $R_{M}$ [45].

The ECAL barrel (EB) covers up to $|\eta|<1.479$ and is comprised of 61,200 lead tungstate $\left(\mathrm{PbWO}_{4}\right)$ crystals. The crystals measure in $0.0174 \times 0.0174$ in $\eta-\phi(22 \times 22$ $\mathrm{mm}^{2}$ ) at the front of the crystal, and $26 \times 26 \mathrm{~mm}^{2}$ in the crystal rear. The crystals are $230 \mathrm{~mm}$ in length, corresponding to a radiation length of $25.8 X_{0}$. The crystals are mounted onto a thin-walled fiberglass cavity and makeup one submodule. A submodule is made up of ten crystals. Groups of submodules group together to form modules, and four modules makeup one supermodule. Each supermodule extends $20^{\circ}$ in $\phi$, resulting in ten supermodules per half-barrel for a total of 36 modules in EB. The artist rendering in Fig. 2.4 shows how the supermodules are configured within the Electromagnetic Calorimeter.

Each supermodule is placed within EB such that the central axis of the crystals are tangential to a circle of radius $66.7 \mathrm{~mm}$. This results in a $3^{\circ}$ tilt in $\eta-\phi$ to accommadate the $6 \mathrm{~mm}$ crack between each supermodule. This off-pointing effect ensures that CMS detects the maximum amount of electromagnetic particles and minimizes particle loss because of cracks in the detector. A visualization of this off-pointing is shown in Fig. 2.5 .

The barrel region of ECAL uses Hamamatsu type S8148 reverse structure avalanche photodiodes (APDs) that were especially developed for CMS. Each ADP has an active area of $5 \times 5 \mathrm{~mm}^{2}$. Two APDs are mounted behind each crystal in the barrel region. 


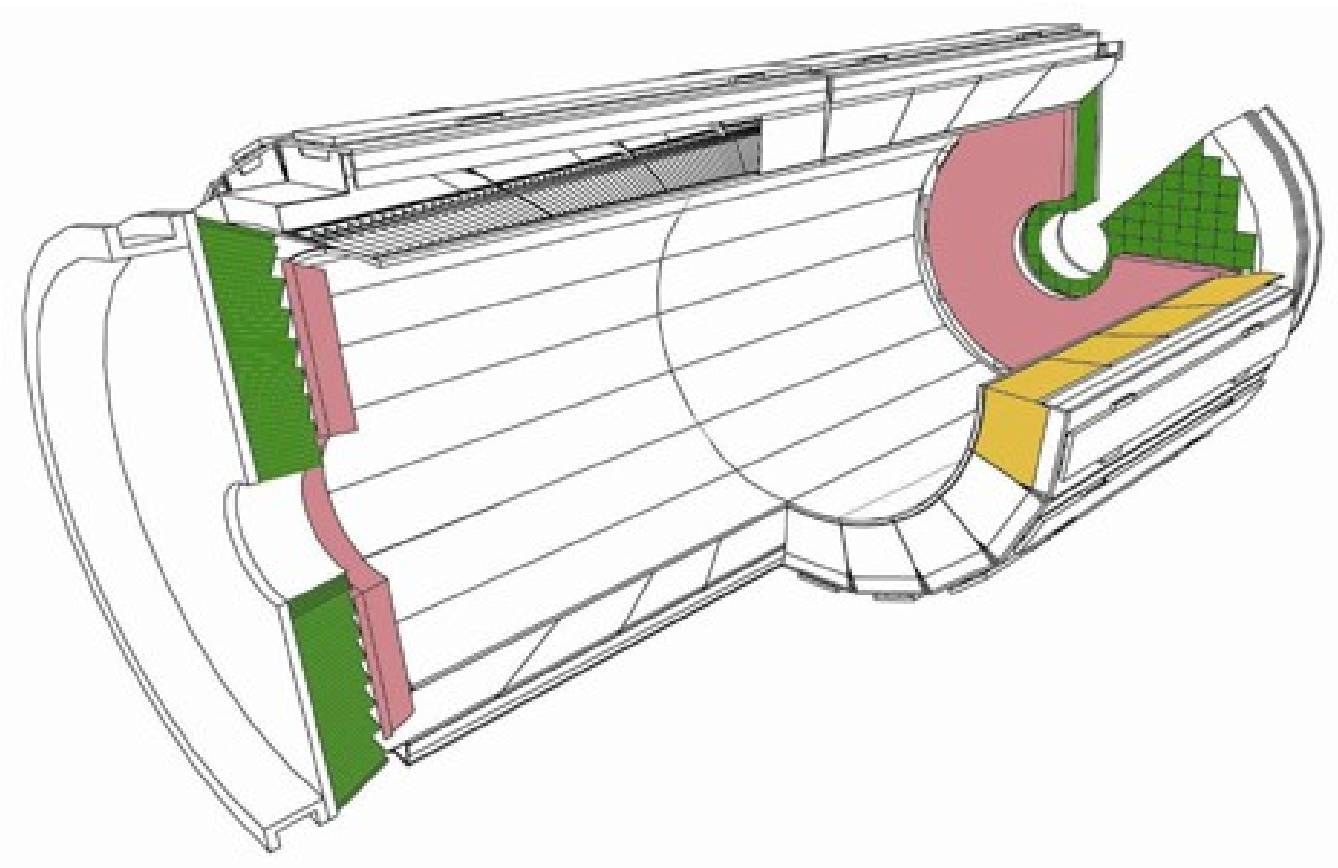

Figure 2.4: Artist rendering of ECAL. The EB modules are horizontal in the central region of ECAL. Each endcap is comprised of 2 'Dees', which get their name from the D-like shape it resembles [12].

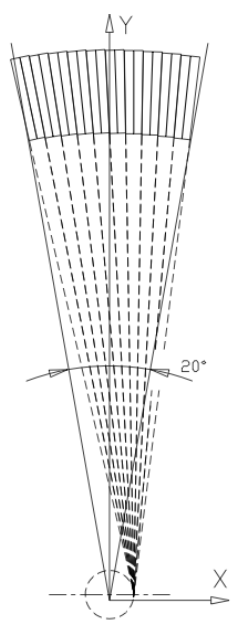

Figure 2.5: Off-pointing geometry for the EB supermodules. The circle has a radius of $66.7 \mathrm{~mm}[12]$. 
The ECAL endcap (EE) covers a pseudorapidity range of $1.479<|\eta|<3.0$ and is comprised of 7324 crystals in each endcap. Each endcap is $3.144 \mathrm{~m}$ away from the IP, this location is the result of the endcaps movement towards the IP when the 3.8 Tesla magnetic field of the solenoid is active. The crystals are arranged in units of $5 \times 5$ crystal arrays in $\mathrm{x}-\mathrm{y}$ within a carbon fiber cavity. Each endcap is comprised of two halves, known as 'Dees' because of their D-like shape. Each Dee is made up of 138 supercrystals (a $5 \times 5$ crystal array in $\mathrm{x}-\mathrm{y}$ ) and 18 special partial crystals on the inner and outer circumferences. The supercrystals are arranged in an $x-y$ grid.

The endcap region in ECAL uses vacuum phototriodes (VPTs), a type of photomultipler tube (type PMT188) from the National Research Institute of Electrons in St. Petersburg, Russia. These VPTs were especially designed for CMS, they have an anode of very fine copper mesh (with a $10 \mu \mathrm{m}$ pitch) which allows them to properly function within the $3.8 \mathrm{~T}$ magnetic field. Each VPT is $25 \mathrm{~mm}$ in diameter, with one mounted behind each crystal in EE. The response of the VPTs is slightly reduced and varies with the angle of the VPT with respect to the field over the range of angles relevant to $\mathrm{EE}\left(6^{\circ}-26^{\circ}\right)$. The mean response of the VPTs in a $4 \mathrm{~T}$ magnetic field at an axis angle of $15^{\circ}$ is $94.5 \%$ of that with no magnetic field present.

The ECAL resolution was measured during test beams. The energy resolution, measured by fitting a Gaussian function to the reconstructed energy distributions, is parameterized as a function of energy:

$$
\frac{\sigma}{E}=\frac{S}{\sqrt{E}} \oplus \frac{N}{E} \oplus C
$$

where the $\oplus$ represents a sum in quadrature. The constants, $S=2.8, N=.125$ and $C=.30$ are the stochastic, noise and constant terms, respectively. The ECAL resolution shown in Fig. 2.6 was measured to be less than $0.50 \%$ for a $50 \mathrm{GeV}$ particle. 


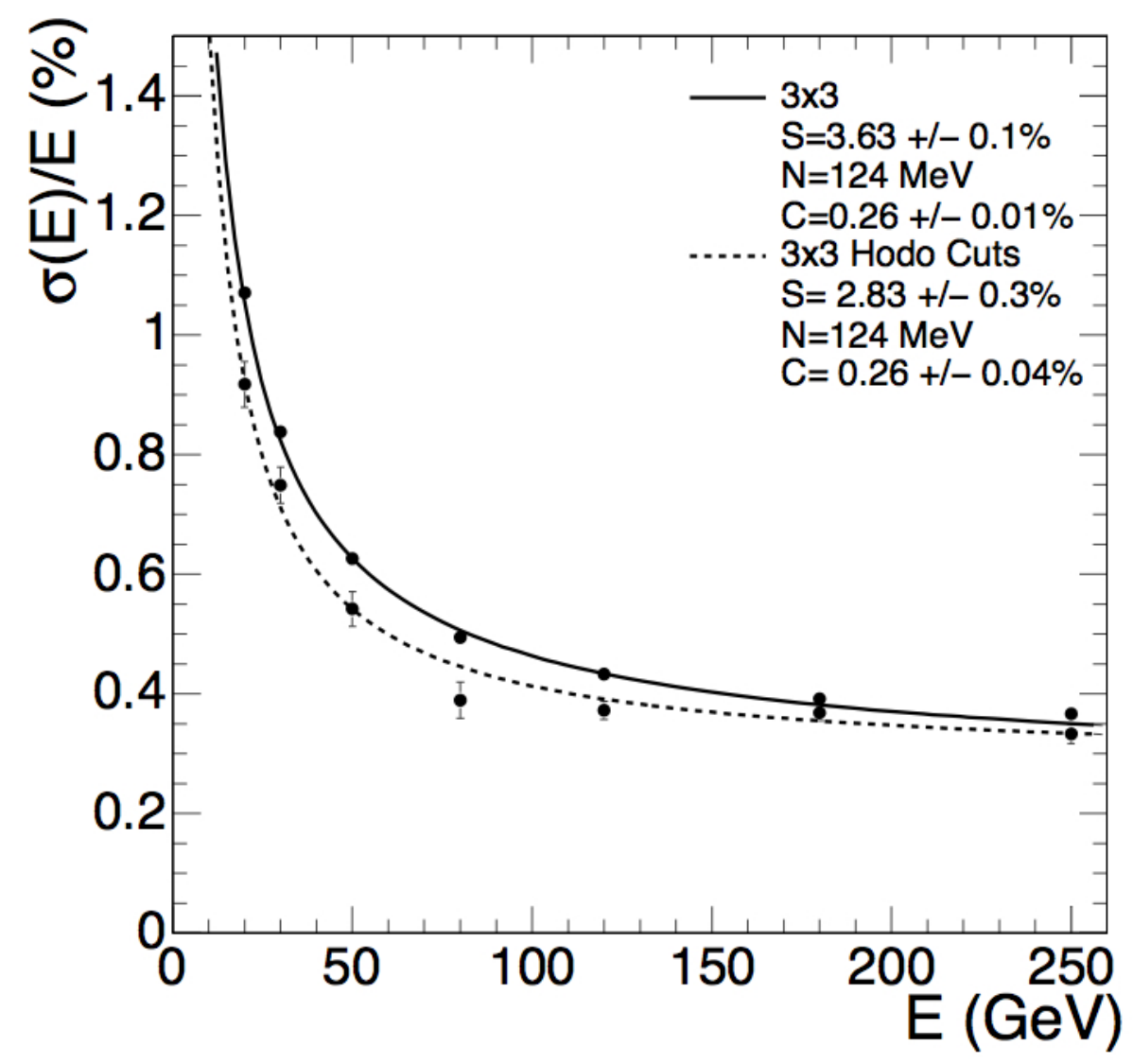

Figure 2.6: Measured ECAL resolution from a test beam [4].

\section{The Hadronic Calorimeter}

The Hadronic Calorimeter sits between ECAL and the superconducting solenoid magnet. The main objective of HCAL is to identify hadronic jets and missing transverse energy $\left(E_{T}^{\text {miss }}\right)$.

Similar to electromagnetic objects, hadrons can interact with the material they traverse and form particle showers. Hadronic showers form at CMS as a result of the hadronic object interacting with absorber plates placed in between scintillating tiles. Unlike electromagnetic showers, hadronic shower formation varies greatly and can take a much longer radiation length to fully record all its energy. The HCAL at CMS is a sampling calorimeter, consisting of alternating brass alloy plates and scintillating 
tile. When a hadron interacts with one of the alloy plates it produces a hadronic shower which is then recorded by the preceding scintillating tile. Immediately following the scintillating tile is another absorber plate in which the already produced hadronic shower produces additional showers which are recorded by the adjacent scintillating tile. The scheme in Fig. 2.7 shows the 17 layers that make up the barrel component of the Hadronic Calorimeter.

The HCAL barrel (HB) covers a range of $|\eta|<1.4$ and surrounds the barrel region of the Electromagnetic Calorimeter. The HB consists of two half barrels, each of which is composed of 18 identical wedges $20^{\circ}$ wide in $\phi$. Each wedge is made up of alternate layers of flat brass alloy absorber plates and active plastic scintillator tiles, with the inner and outer most layers of the wedge made up of stainless steel. The first scintillator layer in HB is roughly double the thickness in order to sample low energy showering particles due to the support between the hadronic and electromagnetic calorimeters. The layer thickness of the scintillator tile and brass alloy vary by layer number. Table 2.2 shows the thickness of each HB wedge layer. Each scintillator tiles are $0.08 \times 0.087$ in $\eta-\phi$. Each tile is furnished with a single wavelength shifting fiber which is intertwined with clear fibers that run down the length of the HB half barrel and connect to pixelated hybrid photodiodes (HPDs) mounted at the end of the barrel region of the Hadronic Calorimeter.

Table 2.2: Scintillator tile and brass alloy thickness for each layer in an HB wedge [4].

\begin{tabular}{|c|c|c|}
\hline Layer(s) & Scintillator Thickness $(\mathrm{mm})$ & Brass Alloy Thickness $(\mathrm{mm})$ \\
\hline 0 & 9 & 61 \\
$1-8$ & 3.7 & 50.5 \\
$9-14$ & 3.7 & 56.5 \\
$15-16$ & $3.7 \mathrm{~mm}$ & 75 \\
\hline
\end{tabular}

The HCAL endcap (HE) interlocks with HB, overlapping with tower 16 as shown in Fig. 2.7 and covers $1.3<|\eta|<3.0$. The wedges that makeup HE are similar 
in geometry to the ones present in HB, except that HE wedges are composed of 19 layers, alternating brass alloy and scintillator tiles. The brass alloy plate is $78 \mathrm{~mm}$ thick while the scintillator tile is $3.7 \mathrm{~mm}$. For $1.3<|\eta|<1.74$ the tiles are the same size as in HB and for $|\eta|>1.74$ sizing in $\eta-\phi$ varies with respect to tower number. For more information on the tile sizes for each HE tower refer to [4].

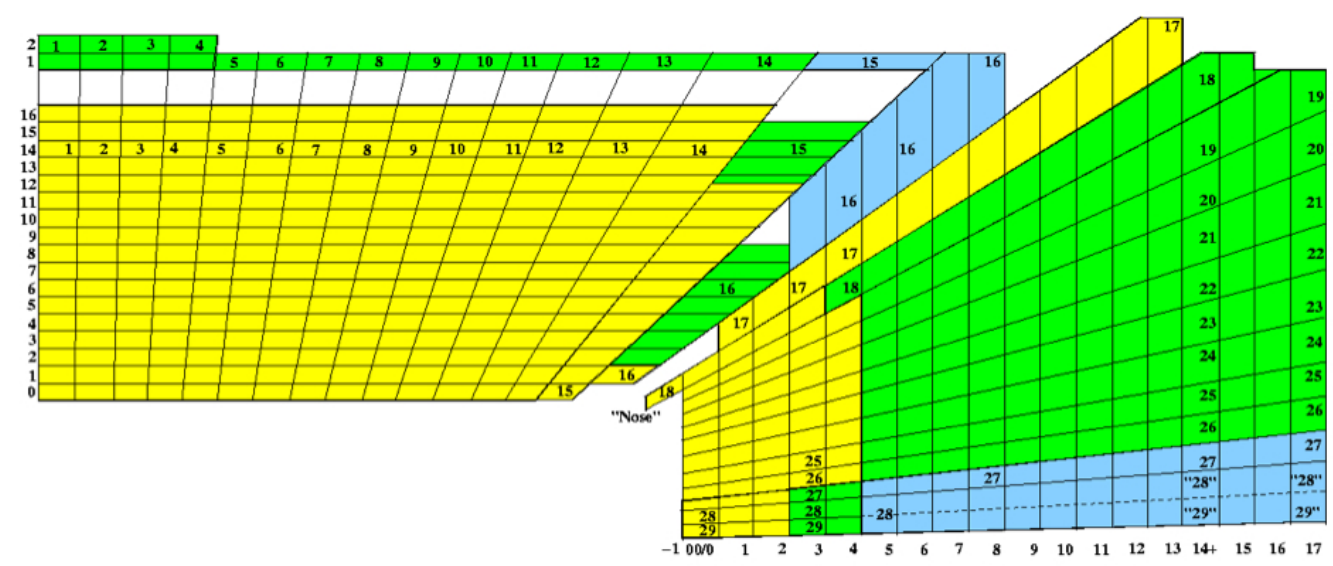

Figure 2.7: Schematic of HB and HE towers. HB and HE overlap at tower 16 [4].

The HCAL Forward (HF) calorimeters are located $11.2 \mathrm{~m}$ from the IP and is composed of steel absorbers embedded with long and short quartz fibers, providing a fast collection of Cherenkov light. Each HF module is made up of 18 wedges with the quartz fibers running parallel to the beam axis $(z)$ and in alternating in size at a separation of $5 \mathrm{~mm}$. The fibers are collected at the end of the module and are readout with phototubes.

\subsubsection{The Trigger System}

Recording and saving every collision occurring at the LHC is an impossible feat with the current technology available. The two-tiered trigger system at CMS is designed to quickly process events from $p p$ collisions and keep only the most interesting events for analyzing offline. 


\section{The Level-1 Trigger}

The LHC expects $\approx 10^{9}$ interactions/sec, however, only $10^{2}$ events/sec are selected for further processing due to limited storage capacity. The Level-1 (L1) Trigger system is the first step in selecting the most interesting events for further analysis. It uses local information from the muon system as well as the calorimeters.

The time it takes for signal in the CMS detector to journey through the electronics to the L1 housing unit is quite small, totaling $3.7 \mu s$. Data are held in buffers while the L1 trigger analyzes the current data in its possession to make a decision as to whether that event will move on to the next level (roughly 1 out of 1000 events are kept).

The $\mathrm{L} 1$ trigger takes about $1 \mu \mathrm{s}$ to decide if the event is held for further processing. These trigger objects are formed by reduced granularity and resolution data seen in trigger towers dispersed throughout the calorimeters and the muon system. The L1 system has an estimated rate of $16 \mathrm{kHz}$ for all the triggers developed for various analyses.

A variety of L1 triggers are available at CMS that fire when such objects as hadronic objects, muons and electromagnetic objects are detected. Since the focus of this analysis are photons, only triggers associated with electromagnetic objects are discussed. An ECAL trigger tower is formed by $5 \times 5$ crystal arrays divided into five strips along the $\phi$ direction. The energy deposits in the trigger tower are summed, giving the transverse energy $\left(E_{T}\right)$ of the tower. In addition, the HCAL energy deposit directly behind the ECAL trigger tower is required to be $<5 \%$. Both requirements create what is called the trigger primitive, which is then used by the L1 trigger to identify electromagnetic objects in ECAL [46]. The four highest energetic candidates from ECAL are sent for a final decision as to whether the event has a candidate passing 
the L1 trigger. A candidate must have $E_{T}>15 \mathrm{GeV}$ in order to be accepted by the L1 trigger for further processing.

\section{High Level Trigger}

Once it passes the L1 trigger, the High Level Trigger (HLT) confirms that the event has the necessary requirements to move on to the next step. Similar to the L1 trigger, there are a number of HLT paths used for different physics objects, but only the HLT path used for this analysis is discussed. The HLT used for this analysis requires an electromagnetic object with an $E_{T}$ threshold higher than required in the L1 trigger. The trigger used is a single photon trigger, meaning that at least one photon in the event must meet the $E_{T}$ threshold. Once the candidates pass the L1 and HLT, reconstruction of particles for analysis can begin. Chapter 4 has further discussion on the triggers used for this analysis.

\subsection{The CMS Computing Infrastructure}

As a consequence of the size of the CMS collaboration and its members spanning all over the globe, there is a need for a computing infrastructure that will allow CMS to store data in a permanent location, transfer it throughout various parts of the globe and process it for further analysis. The computing hierarchy is broken into 'Tiers', named Tier-0, Tier-1, Tier-2, and Tier-3 centers which are computing facilities used to store and process data, generate Monte Carlo samples and provide computing resources for analyzers.

The first stop for data is the Tier-0 (T0), located at CERN. The tasks of the T0 are as follows:

- Accepts RAW data passing the CMS Data Acquisition and Trigger System (TriDAS) 
- Organizes the data into primary datasets based on trigger information

- Saves data collected to a permanent storage system (tape)

- Performs reconstruction on data and reduces data into Analysis Object Data (AOD)

- Performs calibration on data to obtain any constants needed to run the reconstruction

The T0 site does not offer any computing resources for analysis as its sole purpose is to collect and organize data from the detector. The data are then transferred to at least two Tier-1 (T1) sites where a copy of the dataset is stored securely in the case the dataset at the $\mathrm{T} 0$ is removed or lost. In addition to working as a backup for the T0 site, the T1 sites allow for re-reconstruction of data when improvements are needed as well as skimming datasets down to a reasonable size for analyzing. It houses data in AOD format, which takes up considerably less disk space compared to RAW and RECO format data (0.05 MB/event compared to $0.25 \mathrm{MB}$ /event for RECO and 1-1.5 $\mathrm{MB}$ /event for RAW). The $\mathrm{T} 1$ sites are located in national laboratories throughout the globe: the United States, Germany, Italy, France, Spain, Taiwan and the United Kingdom.

Tier-2 (T2) sites offer considerable computing resources for researchers. It allows members of the collaboration to analyze data and store files. The T2's are generally located at universities and offer whatever computing resources relevant to the group of users of that T2 site. Monte Carlo production may take place at T2 sites, but must then be transferred to a T1 in order to keep the amount of disk space used to a minimum [47].

Tier-3 (T3) sites are comparable in size compared to T2 and offer much of the same computing resources as a T2 without the lapse time often encountered by connecting 


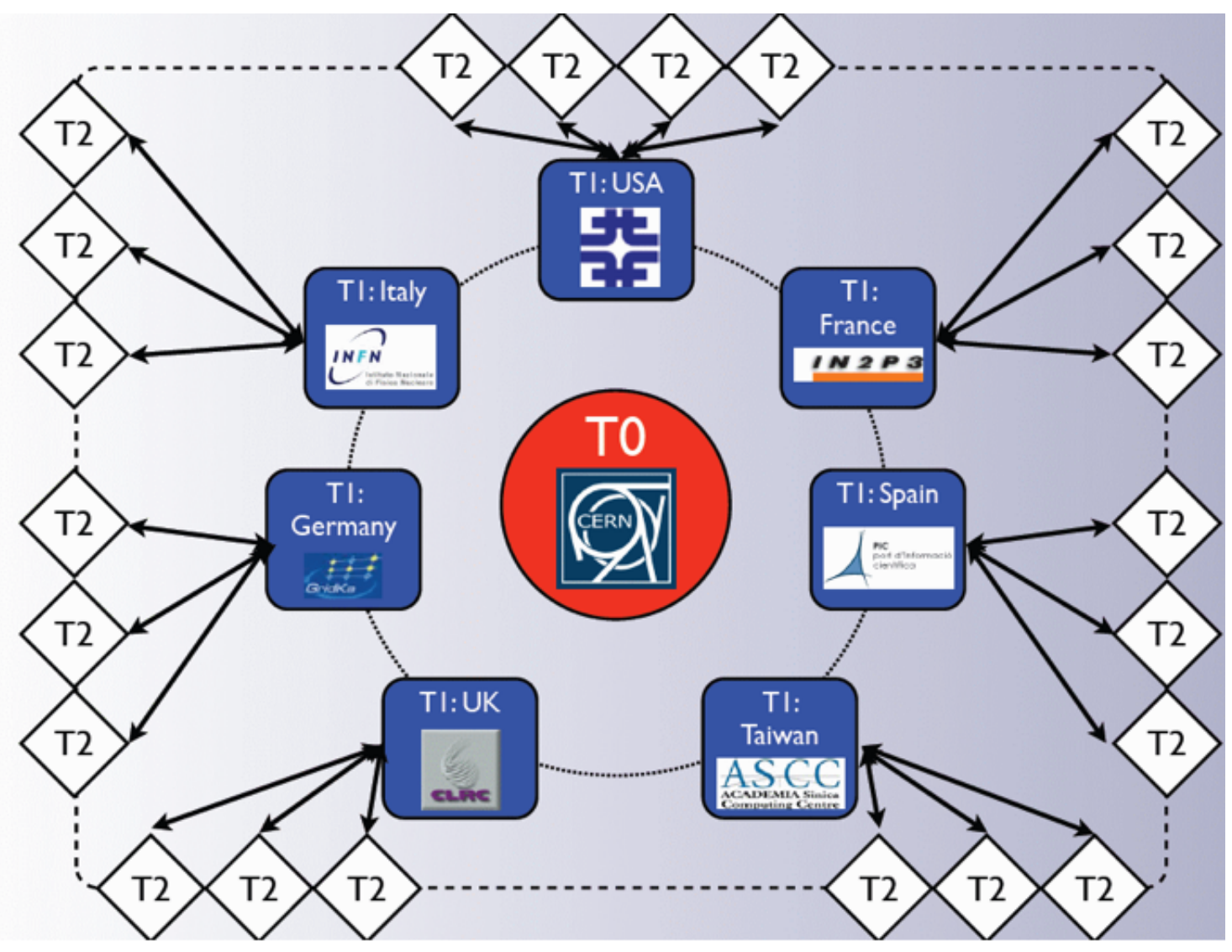

Figure 2.8: CMS Tier organization. 
to a T2. The T3's are usually for the use of members of an institution and can be used for data storage and analysis. Florida International University has a T3 which has been put to extensive use by the particle physics group, and in particular its graduate students.

Members of the CMS collaboration have access to the grid computing infrastructure associated with the experiment through grid interfaces. The grid infrastructure enables members of the collaboration with access to datasets for analysis that are located at various sites throughout the globe. The grid computing infrastructure that stores data and enables analysis on LHC experiments is maintained by members of the collaboration located at institutions and laboratories across the globe. The federation of resources is known as the Worldwide LHC Computing Grid (WLCG) and includes the Open Science Grid (OSG) who supports grid middleware and the grid operations centers in the US [48]. The majority of the resources used by experimenters is maintained by the collaboration in partnership with many computing centers across the globe.

Since it is impossible to contain all the data recorded by the CMS detector in one location it is necessary that researchers still have access to the data regardless of their physical geographical location. The grid enables users to access a dataset located in one part of the globe, run the jobs needed for their analysis and return the output in yet another part of the globe of their choice. The CMS software package used to interface with the WLCG is called the CMS Remote Analysis Builder, or CRAB. CRAB executes jobs in a 'batch-like' fashion and once the jobs are finished the output can either be copied to a storage system of the users choice (they must have an account set up) or returned to the tier site in which the job was executed. These CRAB jobs can be executed from one computing location, run at a different location and copied to another location [49]. The advantage of grid computing is that Other than the 
creation, submission and output collecting done by the user all other tasks performed by the grid are transparent. 


\section{CHAPTER 3}

\section{OFFLINE COMPUTING}

The CMS collaboration has developed its own software to process events and analyze data. The CMS software, CMSSW, was influenced by preceding high energy physics experiments and is written in the $\mathrm{C}++$ programming language which relies heavily on an object oriented framework. The software is utilized for the design, evaluation, construction, and calibration of the detector as well as reconstruction of physics objects (such as electrons, photons and jets) and data analysis. The reconstruction process for photon and jets are the focus of this section.

\subsection{Photon Reconstruction}

As photons traverse ECAL, they will deposit $\sim 94 \%$ of their energy within a $3 \times 3$ crystal array and $\sim 97 \%$ of their energy within a $5 \times 5$ crystal array [12]. Photons may also convert into $e^{-} e^{+}$pairs due to the material present between the IP and the electromagnetic calorimeter. The magnetic field causes the electron-positron pairs to spread further in the $\phi$ direction.

The energy deposits within neighboring crystals are grouped together to form a cluster. A group of clusters are then grouped to form superclusters. Since the crystal arrays differ between EB and EE different clustering algorithms for each region are used: clusters in EB use the hybrid algorithm while clusters in EE use the multi $5 \times 5$ algorithm.

The hybrid algorithm skips the smaller clustering and instead makes superclusters. The algorithm looks for the highest $E_{T}$ crystal above $1 \mathrm{GeV}$ that does not already belong to a supercluster and designates it as the seed crystal. It then looks at the energy deposits of the neighboring crystals in $\eta$ and forms a $1 \times 3$ crystal domino in $\phi-\eta$ phase space. It extends further in $\eta$ to create a $1 \times 5$ crystal domino $\phi-\eta$ provided 
the crystals energy exceeds $350 \mathrm{MeV}$. If this condition is not met, then the $1 \times 3$ crystal domino remains for further clustering. The hybrid algorithm then continues to look for seed crystals in the $\phi$ direction (17 steps to each side of the domino) and groups the crystals that meet the energy threshold to form a supercluster. Once a supercluster is formed, the algorithm begins to search again for another seed crystal to form more superclusters until there are no more crystals with $\mathrm{E} ; 1 \mathrm{GeV}$.

The multi $5 \times 5$ algorithm begins similar to the hybrid algorithm in that it looks for the highest $E_{T}$ crystal above $180 \mathrm{MeV}$ that is not yet grouped to a cluster and designates it as the seed crystal for the cluster. Different from the hybrid algorithm, the multi $5 \times 5$ then creates an array of $5 \times 5$ crystals around the seed crystal. Neighboring crystals of the $5 \times 5$ array may only join if it is a lone highly energetic crystal, meaning that it has no chance of being grouped with another crystal array. The steps are repeated until all seed crystals form clusters. Superclusters are formed where overlapping clusters are grouped together.

The supercluster's position, $x$, is calculated by taking a weighted mean of each crystal within the supercluster, $W_{i}$, obtained using the logarithm of the crystal energy:

$$
\begin{gathered}
x=\frac{\sum x_{i} W_{i}}{\sum W_{i}} \\
W_{i}=W_{0}+\log \left(\frac{E_{i}}{E_{S C}}\right)
\end{gathered}
$$

where $x_{i}$ is the position of the $i^{t h}$ crystal within the supercluster, $W_{0}$ is a constant, $E_{i}$ is the energy of the $i^{t h}$ crystal within the supercluster and $E_{S C}$ is the energy of the supercluster.

If the energy in HCAL directly behind the supercluster is less than $15 \%$ of the cluster's energy, the supercluster enters the photon collection. 


\subsection{Jet Reconstruction}

Jets are a collimated shower of hadrons produced during the fragmentation process. This behavior is a direct result of confinement, which states that colored partons can not be observed as isolated particles. The reconstruction of charged and neutral hadrons using the Particle-Flow algorithm is discussed followed by the algorithm used to group these objects to form jets [16].

\subsubsection{The Particle Flow Algorithm}

The main sub-detectors used to reconstruct hadrons are the tracking system (for charged hadrons), ECAL and HCAL (for both charged and neutral hadrons). The $\mathrm{PF}$ algorithm begins in the tracking system with measuring a physics object's momentum and direction. The momentum can be measured with great precision in the tracking system due to its high resolution in comparison with the Hadronic Calorimeter. Since the tracking system is closer to the production vertex of the physics object, it can measure its direction well before any deviation due to the magnetic field occurs. An iterative-tracking technique is used in order to achieve a high tracking efficiency while maintaining a low fake-rate. This technique first selects tracks using a stringent track seeding criteria, which are then removed from consideration before the next iteration of track selection occurs. The first three iterations of track selection progressively loosens the track seeding criteria; after each iteration the selected tracks are removed from consideration before the next iteration begins. The forth and fifth iterations relax the constraints on the origin vertex, allowing to reconstruct tracks due to secondary particles from such processes as photon conversions and nuclear interactions with the tracking material.

The next step for both charged and neutral hadrons is calorimeter clustering. The

clustering algorithm is performed independently in each of the following sub-detectors: 
ECAL Barrel, ECAL Endcap, HCAL Barrel, HCAL Endcap, Preshower first layer and Preshower second layer (the preshower precedes the ECAL Endcap and is discussed in more detail elsewhere [4]). The algorithm runs as follows: First identify cluster seeds, which are formed by calorimetric cells that meet or exceed an energy threshold, then use the cluster seed to form topological clusters, in which cells neighboring the cluster are combined to it if it exceeds an energy threshold (this threshold varies by sub-detector but has a value of two standard deviations of the electronic noise). The position and energy of the cluster is then determined by measuring the distance between the seed and the remaining cells that make up the cluster and summing the energy of the cells within the cluster, respectively.

The physics objects reconstructed in the tracking system and the calorimeters must now be combined in order to form a single particle flow object, which is done through the use of a link algorithm which combines information from the tracking system objects and the calorimeter objects (discussed in Chapter 2). The last measured position of a track is extrapolated to both layers of the preshower, followed by ECAL (up to a depth consistent with the longitudinal length of an electron shower profile) and finally HCAL (up to a depth of one interaction length, consistent with a typical hadronic shower). If the extrapolation of the track position is within any of the cluster boundaries in the preshower, ECAL or HCAL, that track is then linked to that cluster. Clusters from ECAL and HCAL may also be linked together if the cluster boundary of the finer granulated calorimeter overlaps the less granulated calorimeter. For example, if the preshower cluster overlaps an ECAL cluster then they are linked; similarly, if the ECAL cluster overlaps an HCAL cluster then they are also linked. Links between the tracking system and the tracker in the muon system are also done (see Ref. [16]), but since muons are not studied in this analysis it is not discussed. 


\subsubsection{The Anti-k $\mathrm{k}_{T}$ Algorithm}

Similar to the $\mathrm{k}_{T}$ [50] and the Cambridge/Aachen [51] jet finding algorithms, the separation distance in the anti- $\mathrm{k}_{T}$ algorithm is defined as:

$$
d_{i j}=\min \left(k_{t i}^{2 p}, k_{t j}^{2 p}\right) \frac{\Delta_{i, j}^{2}}{R}
$$

where $\Delta_{i j}^{2}=\sqrt{\left(y_{i}^{2}-y_{j}^{2}\right)+\left(\phi_{i}^{2}-\phi_{j}^{2}\right)}$ and $k_{t i}, \mathrm{y}_{i}$ and $\phi_{i}$ are defined as the transverse momentum, rapidity and azimuthal angle for the $i^{\text {th }}$ particle, respectively. Consider an event in which there are several hard particles clearly spaced out and many soft particles throughout. The distance $d_{1 i}$ between hard particle 1 and soft particle $i$ is determined by $\Delta_{i, j}^{2}$ as well as $k_{t 1}$. Soft particles equidistantly spaced will have a larger $d_{i j}$, causing soft particles to prefer clustering to hard particles before they cluster with each other. A hard particle with no other hard particle neighbors within a radius of $2 R$ will result in that hard particle obtaining all the soft particles surrounding it within a radius of $R$ and create a perfectly conical jet.

If there is a hard particle 2 neighbor within $R<\Delta_{i j}<2 R$ of the initial hard particle 1 then there will be two jets. If $k_{t 1}>>k_{t 2}$ then hard particle 1 will be conical while hard particle 2 is partly conical, with the area of overlap between 1 and 2 contributing towards jet 1 . If $k_{t 1}=k_{t 2}$ neither of the hard particles will be conical and the overlapping area is equally split between the hard particles [13]. The distributions in Fig. 3.1 show a visualization of these situations.

\subsubsection{Jet Energy Scale}

The jet energy deposited in the CMS detector is usually different from the true jet energy obtained by the particles due mainly to non-linear and non-uniform response of the detector. In addition, electronic noise and pile-up can further distort the true 

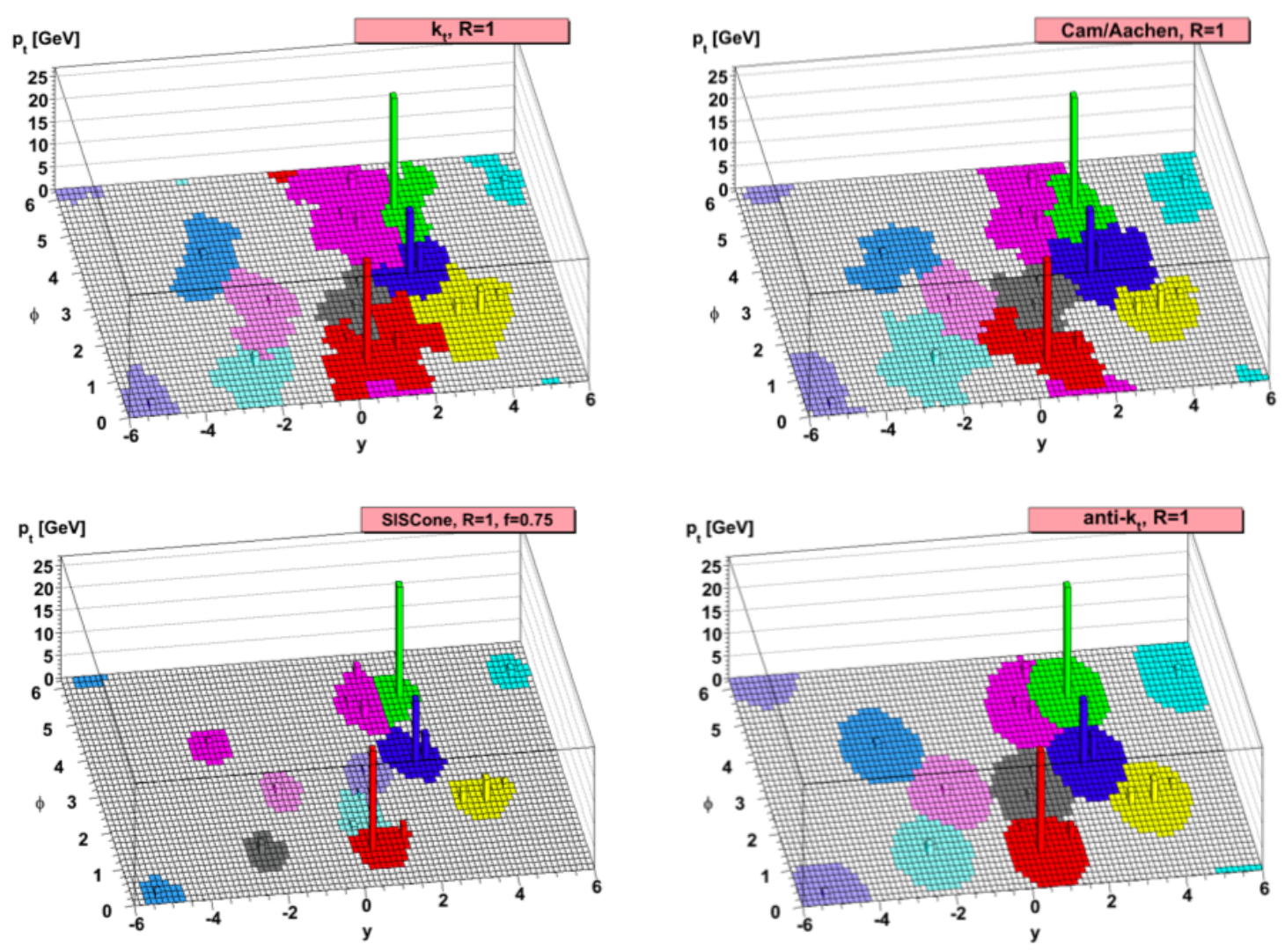

Figure 3.1: Monte Carlo generated particles with soft particles surrounding hard particles using 4 jet finding algorithms [13].

jet energy. A series of corrections are made in order to obtain the true jet energy: An offset correction, $E_{\text {offset }}$, diminishes any excess energy deposits due to electronic noise and pile-up; a relative correction $C_{\text {Rel }}$, accounts for any variation in the jet response as a function of $\eta$ and an absolute correction; and $C_{A b s}$, accounts for any variation in the jet response as a function of $p_{T}$. The expression including these corrections for the jet energy is:

$$
E_{\text {corrected }}=\left(E_{\text {uncorrected }}-E_{\text {offset }}\right) \times C_{\text {Rel }}\left(\eta, p_{T}^{\prime \prime}\right) \times C_{A b s}\left(p_{T}^{\prime}\right)
$$

where $p_{T}^{\prime \prime}$ is the jet's corrected $p_{T}$ due to the offset, and $p_{T}^{\prime}=p_{T}^{\prime \prime} \times C_{\text {Rel }}\left(\eta, p_{T}\right)[14]$. 

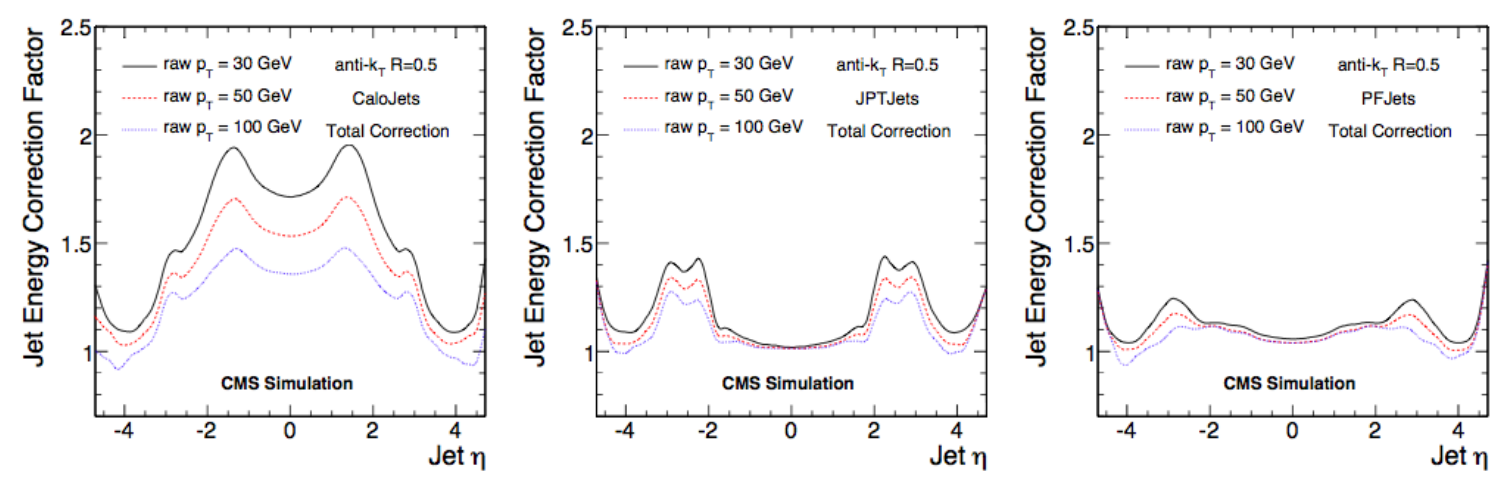

Figure 3.2: Jet Energy Correction Factor, $\mathrm{E}_{\text {corrected }}$, as a function of Jet $\eta$ for CALO jets, Jet-Plus-Track Jets (JPT) and Particle Flow Jets (used in this analysis) [14. 


\section{CHAPTER 4}

\section{EVENT SELECTION}

\subsection{Monte Carlo Samples}

Monte Carlo (MC) refers to events generated through the use of computational algorithms. Looking at the generated events however is not enough, since what is important is how the particles behave within the CMS detector. Generated events are passed through a simulator mimicking the conditions within the CMS detector. Simulation of the detector is done using GEANT4 [52]. Once events have passed the detector simulation the detector's response to the particles passing through it must be reproduced, in a process known as digitization.

Event generated samples are used to find the best set of cuts to select the most signal candidates in data while keeping the amount of background selected to a minimum. Cut values of these kinematic variables are selected by comparing their distributions between signal and background and selecting a cut value that effectively separates them.

This analysis uses the PYTHIA [53] event generator which contains theoretical models for hard and soft interactions as well as partonic showers (both in the initial and final state), fragmentation and decays. The fragmentation is simulated using the Lund string model, in which strings represent the distance between outgoing partons of an interaction. As the partons gain further distance between them the string breaks, representing the production of a new parton-antiparton pair. The process of pair production continues until the hadron's mass is on-shell, meaning that the system obeys the classical laws of motion. The 2011 Summer production of PYTHIA MC for the collaboration is used to compare with data [54]. Signal events are modeled by generated $\gamma+$ Jet events while the background is modeled through QCD Jet events. 
A list showing the MC samples used to model signal and background are shown in Tables 4.1 and 4.2 , respectively.

Table 4.1: $\hat{p_{T}}$ ranges and cross sections of the official MC generated events for the CMS collaboration used to model photons for the analysis.

\begin{tabular}{|c|c|}
\hline$\hat{p_{T}}$ range $(\mathrm{GeV})$ & Cross Section $(p b)$ \\
\hline $30-50$ & $1.669 \times 10^{4}$ \\
$50-80$ & $2.722 \times 10^{3}$ \\
$80-120$ & $4.472 \times 10^{2}$ \\
$120-170$ & 84.17 \\
$170-300$ & 22.64 \\
$300-470$ & 1.493 \\
$470-800$ & $1.323 \times 10^{-1}$ \\
$800-1400$ & $3.481 \times 10^{-3}$ \\
\hline
\end{tabular}

Table 4.2: $\hat{p_{T}}$ ranges and cross sections of the official MC generated events for the CMS collaboration used to model background for the analysis.

\begin{tabular}{|c|c|}
\hline$\hat{p_{T}}$ range $(\mathrm{GeV})$ & Cross Section $(p b)$ \\
\hline $30-50$ & $5.312 \times 10^{7}$ \\
$50-80$ & $6.359 \times 10^{6}$ \\
$80-120$ & $7.843 \times 10^{5}$ \\
$120-170$ & $1.151 \times 10^{5}$ \\
$170-300$ & $2.426 \times 10^{4}$ \\
$300-470$ & $1.168 \times 10^{3}$ \\
$470-600$ & 70.22 \\
$600-800$ & 15.55 \\
$800-1000$ & 1.844 \\
$1000-1400$ & $3.321 \times 10^{-1}$ \\
$1400-1800$ & $1.087 \times 10^{-2}$ \\
\hline
\end{tabular}

The early 2009-2010 data brought to to light the need to tune PYTHIA so that MC properly modeled data [30] by changing the PDF set used and varying how the UE and initial state radiation are modeled. The 'Tune Z2' has been optimized to properly reflect the behavior of the UE observed at the experiment.

\subsubsection{Pile-up Effects}

Pile-up refers to the remaining physics objects present during a collision that originated from the previous collision. These effects need to be accounted for in the MC 
samples used in the analysis in order to properly model the effect present in data. The pile-up is accounted for by looking at the number of primary vertices for each event in both data and MC. One such distribution is shown on the left-hand side in Fig. 4.1. The data distribution is divided by the MC distribution and a constant is obtained, which is used to scale any distribution in $\mathrm{MC}$ with respect to the number of primary vertices in that event in order to properly reflect how that distribution appears with the pile-up effects accounted for. As an example, the number of primary vertices in $\mathrm{MC}$ is corrected using these constants and shown on the right-hand side in Fig. 4.1 to properly reflect that same distribution in data. The constants used to weight events due to pile-up are shown in Table 4.3 .
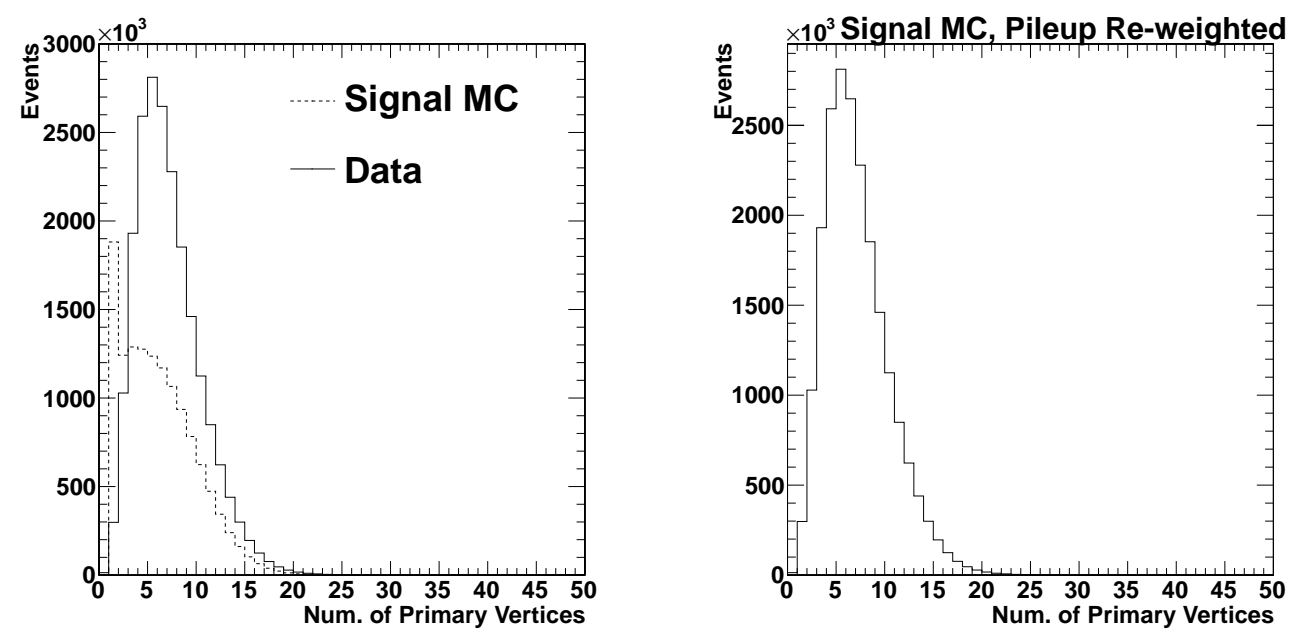

Figure 4.1: Number of primary vertices in data and signal MC (left). The Number of primary vertices in signal MC after corrected for pileup (right).

\subsection{Data Samples}

The data recorded by the CMS detector during the 2011 run correspond to about $5.56 \mathrm{fb}^{-1}$, as shown in Fig 4.2 . The $\mathrm{L} 1$ trigger required at least one electromagnetic object with $\mathrm{E}_{T}>15 \mathrm{GeV}$. As a result of an increase in instantaneous luminosity and the limited trigger bandwidth, various pre-scale factors were introduced for HLT paths with lower $p_{T}$ thresholds. Pre-scale factors are introduced to reduce the use 


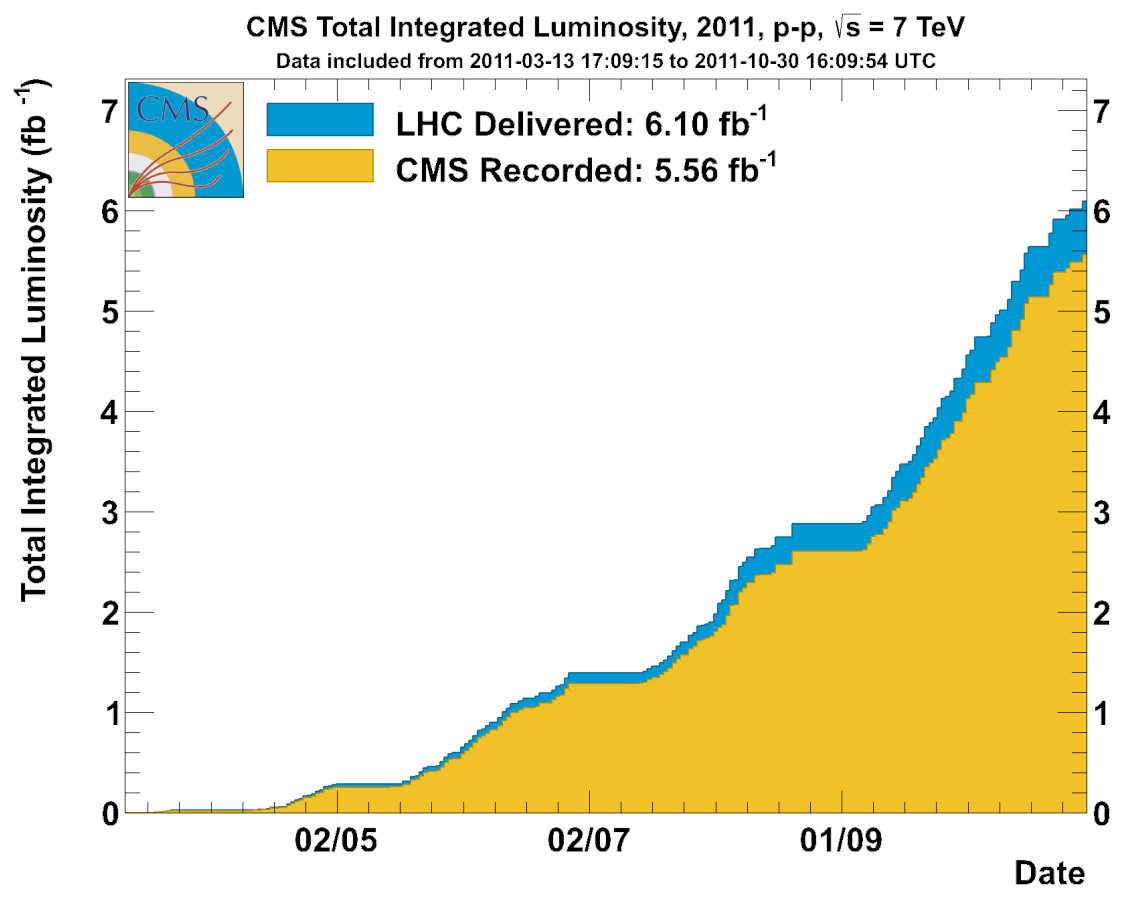

Figure 4.2: The total integrated luminosity delivered (blue) and recorded (yellow) during the 2011 run [15].

of bandwidth during event processing. It reduces the bandwidth usage by reducing the amount of events allowed to pass the trigger(s), for example if the pre-scale is set to 10 , it will only process one event out of every 10 events that satisfy the trigger requirements. The HLT path used in this analysis is HLT_Photon30_CaloIdVL, which accepts events where at least one photon is present with a $p_{T}$ of $30 \mathrm{GeV}$ as the threshold.

The data were collected during two run periods, known as 2011A and 2011B where the instantaneous luminosity was increased. Table 4.4 lists the primary datasets used in the analysis along with their run ranges, total number of events originally present in each sample and the effective luminosity corresponding to the HLT path used for the analysis. 


\subsection{Variables Used for Analysis}

While the L1 and HLT triggers allow the best possible photon candidates into the data sample, the occurrence of background entering is a common issue in any high energy physics analysis. In order to reduce the amount of background and maximize signal, additional cuts need to be applied to candidates.

The cross section of jet production, the background in this study, is about $10^{3}$ times larger than the cross section of prompt photon production. In order to reduce the background, a set of isolation variables are used in data. Prompt photons are expected to have very little activity within their vicinity as opposed to jets, which can have energy showers about their central axis. A candidate's isolation is measured as follows; first an annulus cone is built in $\eta-\phi$ around the candidate, then the $E_{T}$ within that cone is summed. If the $E_{T}$ within the cone is below a given maximum then the candidate is considered to be isolated. The candidate is checked if it is isolated in three regions of the CMS detector: the Tracking system, the ECAL and the HCAL. Fig. 4.4 shows the isolation distributions for signal and background MC. The isolation variables used in this analysis are described in more detail:

- Electromagnetic Isolation $\left(\operatorname{Iso}_{E C A L}\right)$ : The $E_{T}$ sum of the electromagnetic reconstructed hits (ecal rechits) deposited in a hollow cone of $\Delta R=\sqrt{(\Delta \eta)^{2}+(\Delta \phi)^{2}}$ between 0.06 to 0.4 around the candidate's seed crystal, where the inner region is vetoed in order to allow energy deposits of the candidates. Only those ecal rechits which have $E>80 \mathrm{MeV}$ are considered for the sum. A 'footprint' of the electromagnetic particles showering is vetoed along a specified strip 0.04 in the $\eta$ direction.

- Hadronic Isolation $\left(\operatorname{Iso}_{H C A L}\right)$ : The $E_{T}$ sum of the hadronic reconstructed towers (hcal towers) in a hollow cone of $\Delta R$ between 0.15 to 0.4 around the candidate's 
seed crystal. Each hcal rechit must pass an energy threshold of $0.9 \mathrm{GeV}$ in the barrel and $1.4 \mathrm{GeV}$ in the endcap before it can be part of the hcal tower collection.

- Hollow Cone Track Isolation ( $\operatorname{Iso}_{T R K}$ ): The sum of the tracks $p_{T}$ in a hollow cone with $\Delta R$ between 0.04 to 0.4 around the candidate's seed crystal. Only tracks with a $p_{T}>200 \mathrm{MeV}$ are included in the sum.

A correction factor is included in the above isolation variables to account for the pileup conditions observed in data. The following correction is applied to all isolation variables:

$$
I s o^{\text {new }}=I s o^{\text {orig }}-\rho_{\text {event }} \times A_{\text {eff }}
$$

where $I s o^{\text {orig }}$ is the original isolation value, $\rho_{\text {event }}$ is the energy density and $A_{\text {eff }}$ is the effective area obtained by plotting both the isolation variables and $\rho_{\text {event }}$ as functions of the number of primary vertices in the event and taking the ratio of their slopes [55]. The values for $\rho_{\text {event }}$ vary for each event while the values for $A_{\text {eff }}$ are shown in Table 4.5 .

Other variables used to select photon candidates in data are:

- Hadronic Over Electromagnetic (Had/Em): The ratio of energy deposited between HCAL and ECAL in the area traversed by the candidate.

- Veto Pixel Seed Hit: Vetoes any photon candidates that register a hit in the tracking systems pixels.

Since the aim of this analysis is to study the angular distributions in the CM frame, we must also discuss such quantities which include the Lorentz boost in $\eta$ : 


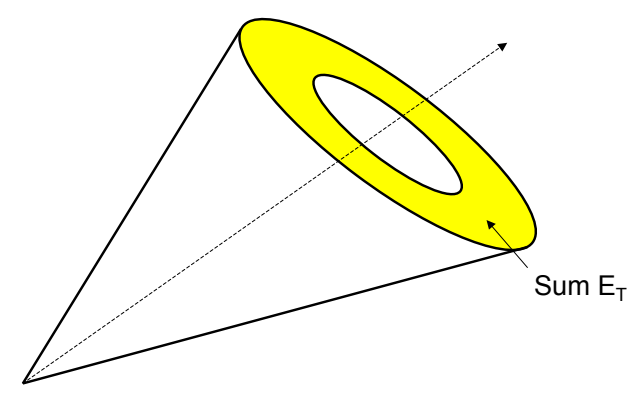

Figure 4.3: Visual depiction of an isolation cone.

$$
\eta_{\text {boost }}=\tanh ^{-1} \frac{p_{z}}{E} \text { where } p_{z}=\sum_{i=\gamma, j e t s} p_{z_{i}} \text { and } E=\sum_{i=\gamma, j e t s} E_{i}
$$

With $\eta^{\gamma}$ and $\eta_{\text {boost }}$, the $\eta$ of the photon candidate in the CM frame, $\eta^{*}$, can be easily solved:

$$
\eta^{*}=\eta^{\gamma}+\eta_{\text {boost }}
$$

Another equivalent form of expressing $\eta^{*}$ in terms of $\eta^{\gamma}$ and $\eta^{\text {jet }}$ is:

$$
\eta^{*}=\frac{\eta^{\gamma}-\eta^{j e t}}{2}
$$

where $\eta^{\text {jet }}$ is taken as the leading jet in the event. 

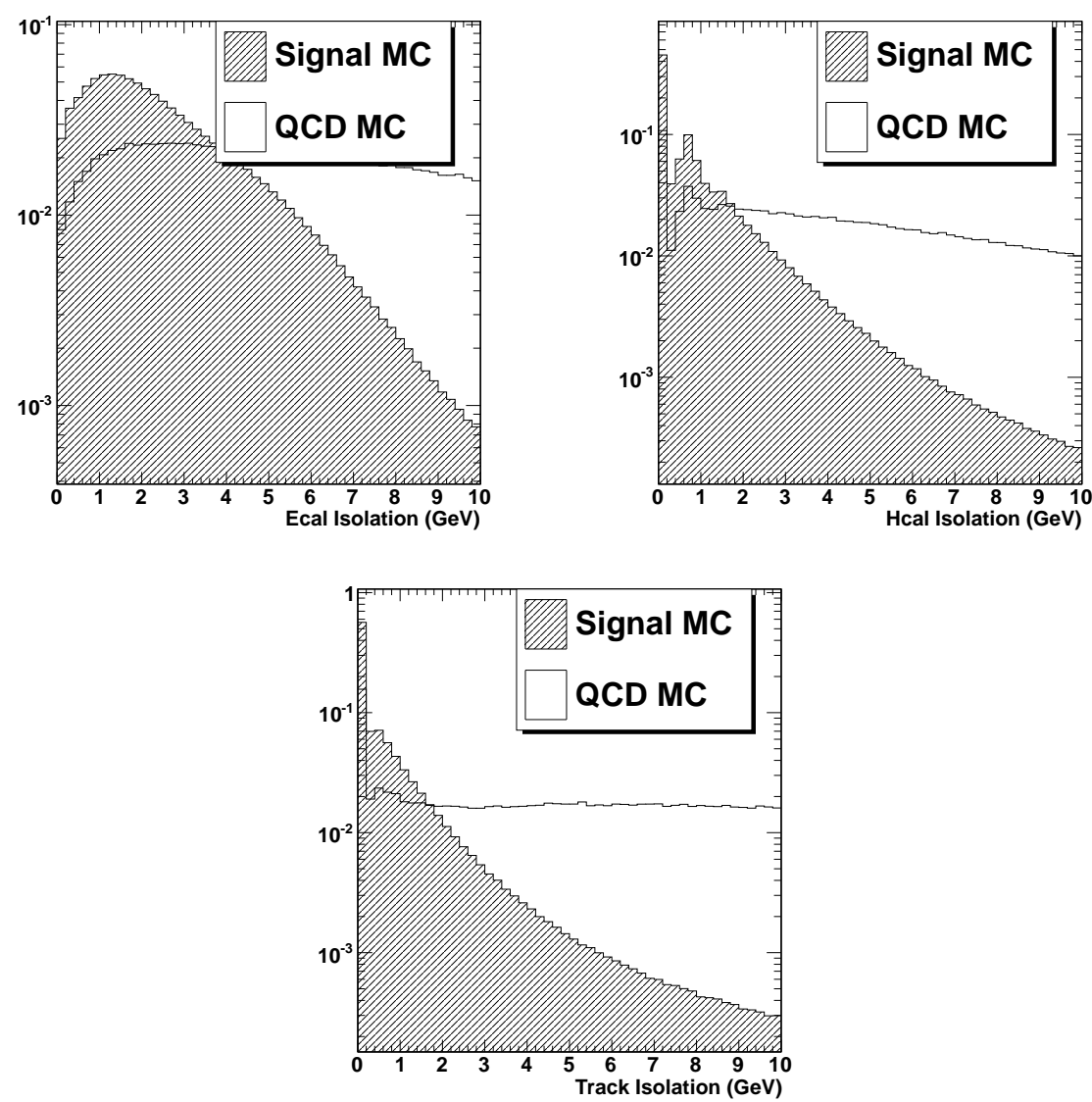

Figure 4.4: Distributions of the following photon candidate kinematic variables in which cuts are made to select signal candidates: Ecal Isolation on the upper-left, Hcal Isolation on the upper-right side, Hollow Cone Track Isolation on the bottom.

\subsection{Event Quality Cuts}

Once events pass the L1 trigger and HLT path, they are required to have a well defined vertex in terms of the distance the primary vertex is from the IP on the $z$-axis, $z_{I P}$, and the minimum amount of reconstructed vertices, $N_{\text {dof }}$, to ensure a hard collision has occurred [56]:

- $\left|z_{I P}\right|<24 \mathrm{~cm}$

- $N_{\text {dof }}>4$ 
Events are required to have at least one photon candidate accompanied by at least one jet per event with both particles meeting their respective $p_{T}$ threshold and $\eta$ requirement. The following set of cuts are placed on the leading photon and jet(the highest $p_{T}$ candidate in the event within the allowed $|\eta|$ region):

- $p_{T}^{\gamma}>40 \mathrm{GeV}$

- $\left|\eta^{\gamma}\right|<1.4442$

- Electromagnetic Isolation $(0.06<\Delta R<0.4)<4.2+0.006 p_{T}^{\gamma}$

- Hadronic Isolation $(0.15<\Delta R<0.4)<2.2+0.0025 p_{T}^{\gamma}$

- Hollow Track Isolation $(0.04<\Delta R<0.4)<2.0+0.001 p_{T}^{\gamma}$

- $\mathrm{Had} / \mathrm{EM}<0.05$

- Veto Pixel Seed Matching (in order to eliminate any electrons that were misreconstructed as a photon)
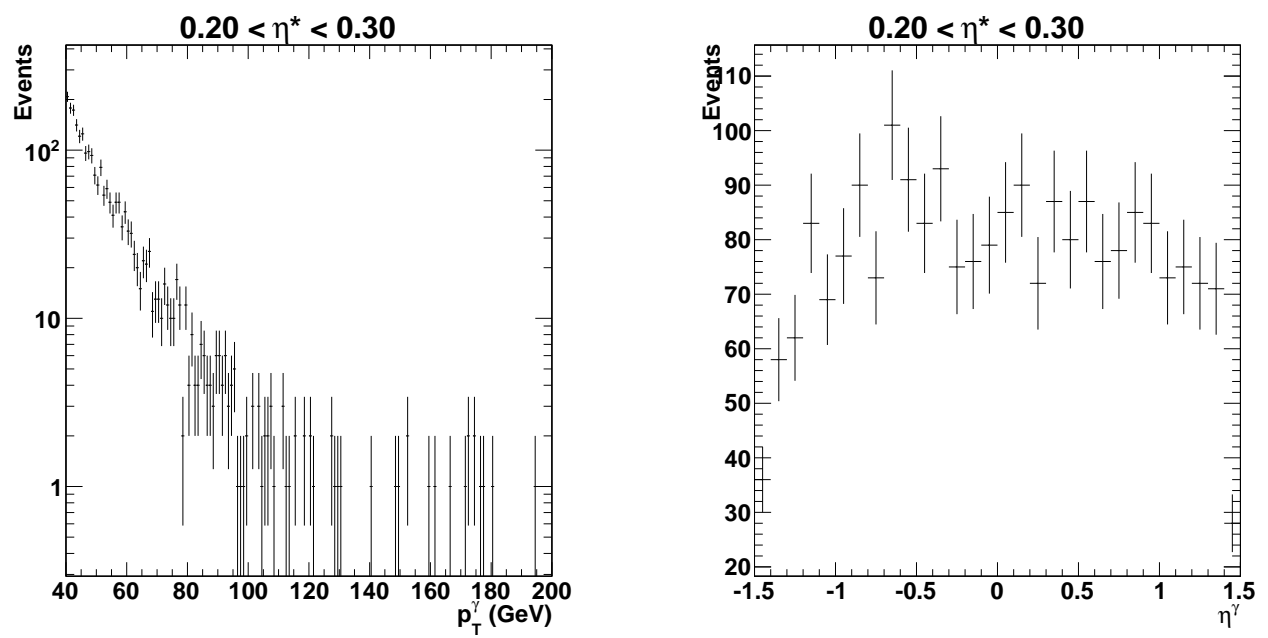

Figure 4.5: The $p_{T}$ distribution of photon candidates (left) and the $\eta^{\gamma}$ distribution (right) for photon candidates with $0.2<\left|\eta^{*}\right|<0.3$.

- $\mathrm{p}_{T}^{\text {jet }}>30 \mathrm{GeV}$

- $\left|\eta^{j e t}\right|<2.4$ 
Events in which both the leading photon and jet satisfy the above requirements are selected for analysis while events in which neither the leading photon or jet satisfy the above conditions are excluded. The $p_{T}$ threshold is located at a value where the jet reconstruction is known to be fully efficient. While HCAL extends up to $|\eta|<$ 5.0 , the tracking system only goes to $|\eta|<2.4$ which gives the ability to properly examine the hollow cone isolation variables between photon and jets. A complete set of $p_{T}^{\gamma}, \eta^{\gamma}, p_{T}^{j e t}$ and $\eta^{j e t}$ distributions are available (in Appendix $\mathrm{A}$.
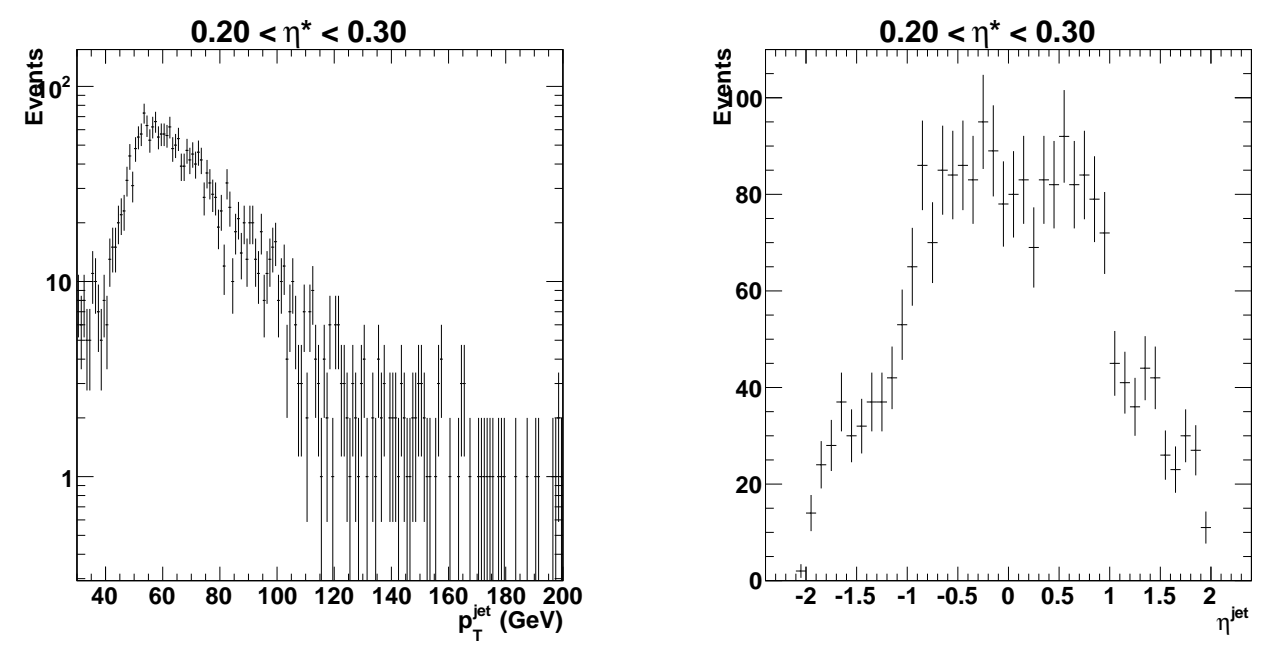

Figure 4.6: The $p_{T}$ distribution of jets (left) and $\eta^{\text {jet }}$ distribution (right) for jet candidates with $0.2<\left|\eta^{*}\right|<0.3$.

\subsection{Efficiencies}

The L1 trigger $p_{T}$ threshold is set considerably lower than the HLT paths in order to be fully efficient at higher $p_{T}$. The $\mathrm{L} 1 p_{T}$ threshold is set at $15 \mathrm{GeV}$ while the HLT path $p_{T}$ threshold is set at $30 \mathrm{GeV}$, allowing for events to occupy a region that is $100 \%$ efficient at the L1 level [57]. Similarly, the $p_{T}$ threshold for this analysis is set well above the threshold within the HLT path, at $p_{T}>40 \mathrm{GeV}$ where the trigger is known to be fully efficient. The distribution in Fig. 4.8 shows that the HLT path used in this analysis is highly efficient. 


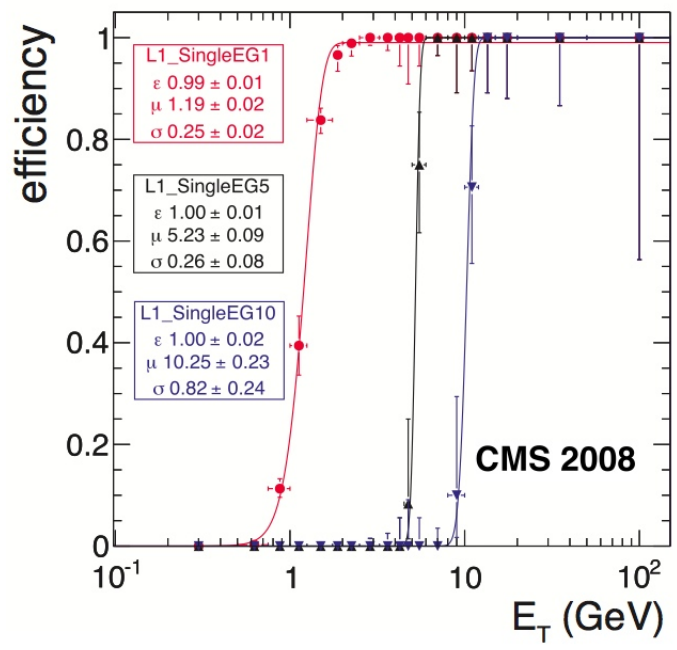

Figure 4.7: Level 1 trigger efficiency as a function of $E_{T}$ [16].

The photon identification efficiency was calculated as a function of $\left|\eta^{*}\right|$. Fig 4.9 shows the efficiency of each individual cut as well as the combined cuts. The denominator is defined as the number of events in which at least one photon and one jet is present with their $p_{T}$ thresholds met and within their allowed $\eta$ region and where the leading photon and jet have a minimum separation of $\Delta R>0.5$ to ensure that the photon is not embedded within the jet. The photon is also required to be matched to a generator-level photon by requiring that it originates from a stable vertex and that its particle ID is 22, the value assigned to photons in event generators [2]. The numerator is defined as those events which pass the denominator requirements and also pass the photon identification cuts. The pass-fail nature of the efficiency calculation dictates the use of binomial uncertainties to calculate the efficiency uncertainty:

$$
\sigma=\sqrt{n p(1-p)}
$$

where $n$ corresponds to the number of trials and $p$ is the probability [58]. For this analysis, Eq. 4.5 takes the form: 


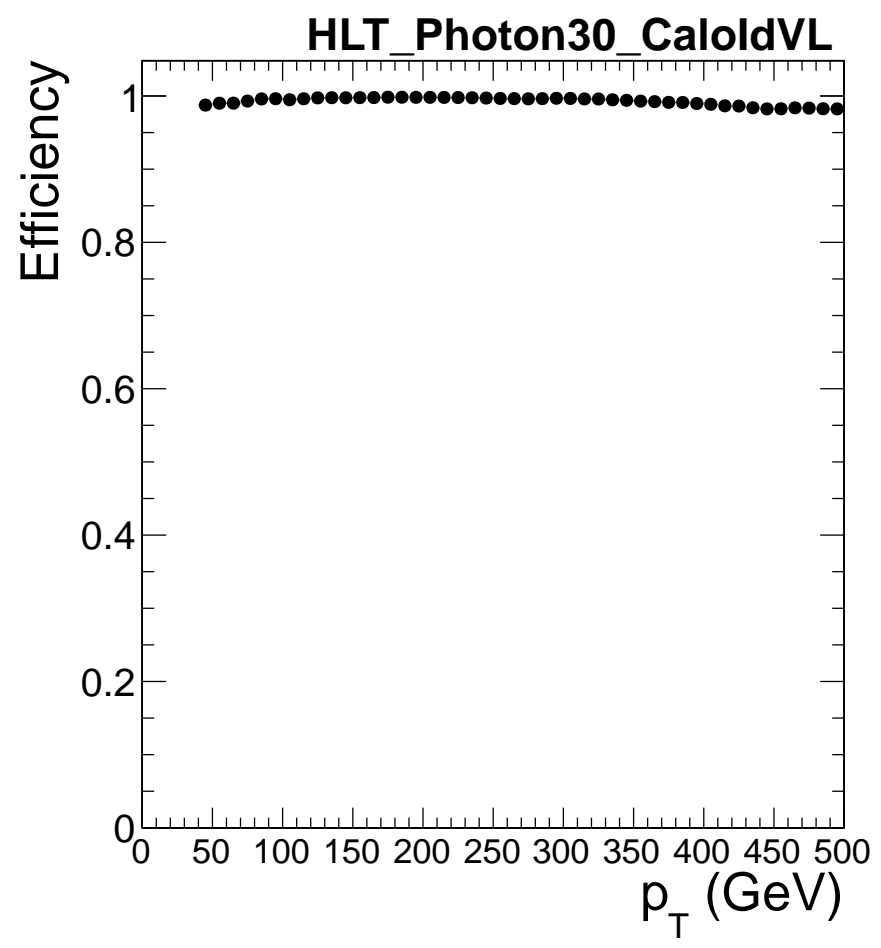

Figure 4.8: HLT efficiency as a function of $p_{T}$.

$$
\Delta \epsilon=\sqrt{\frac{\epsilon(1-\epsilon)}{D}}
$$

where $\epsilon$ is the efficiency calculated and $D$ corresponds to the number of entries in the denominator used to calculate the efficiency. Table 4.6 shows the efficiency values and its corresponding uncertainties. 


\section{S4 PYTHIA}

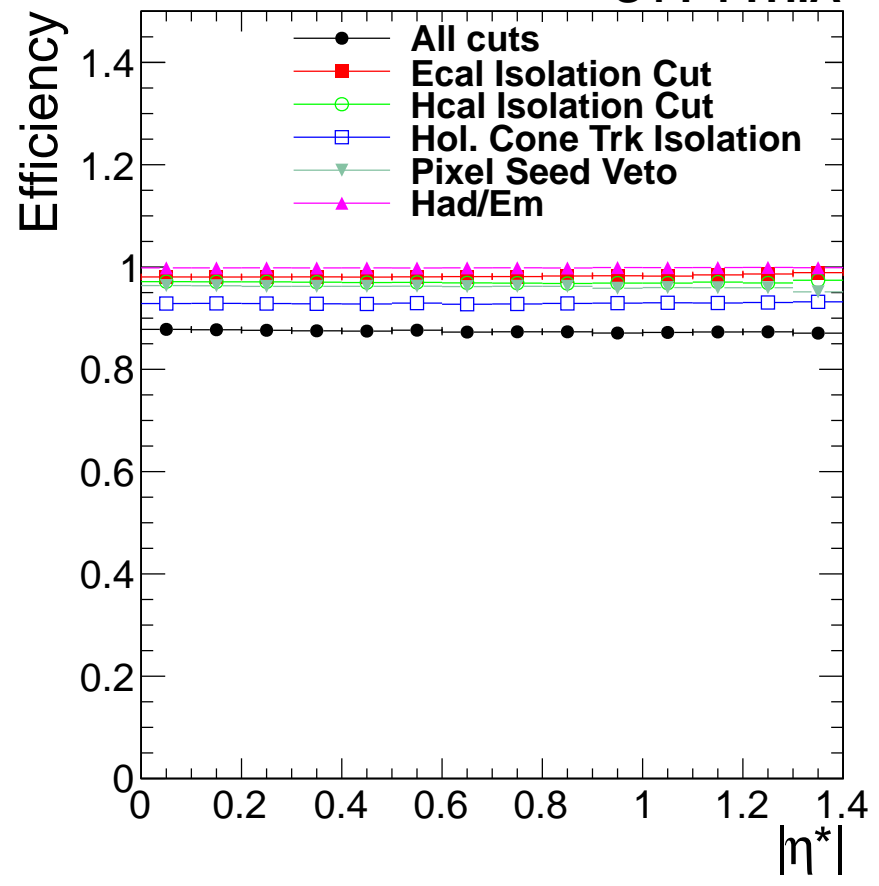

Figure 4.9: Photon identification efficiencies for each individual cut as well as the combined set of cuts. 
Table 4.3: The weights used to account for pileup according to the number of primary vertices a $\mathrm{MC}$ event reports.

\begin{tabular}{|c|c|}
\hline Num. of Primary Vertices & $\frac{\text { Data }}{M C}$ \\
\hline 1 & 28.53 \\
\hline 2 & 0.1583 \\
\hline 3 & 0.8281 \\
\hline 4 & 1.499 \\
\hline 5 & 2.031 \\
\hline 6 & 2.274 \\
\hline 7 & 2.263 \\
\hline 8 & 2.138 \\
\hline 9 & 1.980 \\
\hline 10 & 1.866 \\
\hline 11 & 1.803 \\
\hline 12 & 1.794 \\
\hline 13 & 1.812 \\
\hline 14 & 1.834 \\
\hline 15 & 1.855 \\
\hline 16 & 1.900 \\
\hline 17 & 1.933 \\
\hline 18 & 1.973 \\
\hline 19 & 2.057 \\
\hline 20 & 2.262 \\
\hline 21 & 2.477 \\
\hline 22 & 2.709 \\
\hline 23 & 3.092 \\
\hline 24 & 3.507 \\
\hline 25 & 3.861 \\
\hline 26 & 4.413 \\
\hline 27 & 3.841 \\
\hline 28 & 3.693 \\
\hline 29 & 5.129 \\
\hline 30 & 5.813 \\
\hline 31 & 4.423 \\
\hline 32 & 5.000 \\
\hline 33 & 4.286 \\
\hline 34 & 2.571 \\
\hline 35 & 3.000 \\
\hline 36 & 0.000 \\
\hline 37 & 4.000 \\
\hline
\end{tabular}


Table 4.4: 2011 Datasets used for this analysis. The effective luminosity reflects the amount of data collected from the trigger used in the analysis.

\begin{tabular}{|c|c|c|c|}
\hline Dataset Name & Run Range & Events & Eff. Lumi. (pb $\left.{ }^{-1}\right)$ \\
\hline /Photon/Run2011A-May10ReReco-v1/A0D & $160329-163869$ & $16,323,959$ & 1.26 \\
/Photon/Run2011A-PromptReco-v4/A0D & $165071-168437$ & $35,611,946$ & 1.53 \\
/Photon/Run2011A-05Aug2011-v1/AOD & $170053-172619$ & $11,949,265$ & 0.188 \\
/Photon/Run2011A-PromptReco-v6/AOD & $172620-175770$ & $12,766,763$ & 0.747 \\
/Photon/Run2011B-PromptReco-v1/A0D & $175832-180296$ & $29,743,111$ & 1.18 \\
\hline
\end{tabular}

Table 4.5: Additional correction factors for the isolation variables used in the analysis. The value of $\rho_{\text {event }}$ is obtained on an event-by-event basis. These values only reflect candidates in $\mathrm{EB}$.

\begin{tabular}{|c|c|}
\hline Isolation & $A_{\text {eff }}$ \\
\hline Electromagnetic Isolation & 0.183 \\
Hadronic Isolation & 0.062 \\
Hollow Cone Track Isolation & 0.0167 \\
\hline
\end{tabular}

Table 4.6: Photon identification efficiencies and it's corresponding uncertainty in bins of $\left|\eta^{*}\right|$.

\begin{tabular}{|c|c|c|}
\hline Bin $\left(\left|\eta^{*}\right|\right)$ & $\epsilon$ & $\Delta \epsilon$ \\
\hline 0.05 & 0.8781 & $4.907 \times 10^{-4}$ \\
0.15 & 0.8773 & $5.045 \times 10^{-4}$ \\
0.25 & 0.8763 & $5.317 \times 10^{-4}$ \\
0.35 & 0.8753 & $5.773 \times 10^{-4}$ \\
0.45 & 0.8747 & $6.406 \times 10^{-4}$ \\
0.55 & 0.8765 & $7.282 \times 10^{-4}$ \\
0.65 & 0.8728 & $8.662 \times 10^{-4}$ \\
0.75 & 0.8734 & $1.029 \times 10^{-3}$ \\
0.85 & 0.8733 & $1.243 \times 10^{-3}$ \\
0.95 & 0.8709 & $1.539 \times 10^{-3}$ \\
1.05 & 0.8721 & $1.906 \times 10^{-3}$ \\
1.15 & 0.8730 & $2.394 \times 10^{-3}$ \\
1.25 & 0.8733 & $3.026 \times 10^{-3}$ \\
1.35 & 0.8707 & $3.924 \times 10^{-3}$ \\
\hline
\end{tabular}




\section{CHAPTER 5}

\section{BACKGROUND ESTIMATION}

\subsection{Signal and Background Discrimination}

The precise purity fraction is determined by using a variable which has discrimination power between signal and background. One such variable is $\sigma_{i \eta i \eta}$, defined as the photon candidate's energy-weighted shower spread in the $\eta$ direction, expressed as:

$$
\sigma_{i \eta i \eta}=\frac{\sum_{i=1}^{5 \times 5} w_{i}\left(\eta_{i}-\eta_{5 \times 5}\right)^{2}}{\sum_{i=1}^{5 \times 5} w_{i}} ; \text { where } w_{i}=\max \left(0.47+\ln \frac{E}{E_{5 \times 5}}\right)
$$

where $E$ is the energy of the $i^{\text {th }}$ crystal, $E_{5 \times 5}$ is the energy deposited within a $5 \times 5$ crystal array, $\eta_{i}$ is the $\eta$-position of the $i^{\text {th }}$ crystal and $\eta_{5 \times 5}$ is the $\eta$-position of the seed crystal of the $5 \times 5$ crystal array. The distribution in Fig. 5.1 shows the $\sigma_{i \eta i \eta}$ distribution for signal and background overlaid with data. Photons deposit most of their energy within a $5 \times 5$ crystal array, which roughly spans 0.087 in $\eta$ within ECAL whereas hadronic showers are less predictable and tend to spread further out. Appendix $\mathrm{C}$ shows a complete set of $\sigma_{i \eta i \eta}$ distributions for each $\left|\eta^{*}\right|$ bin.

The signal template was obtained through the MC samples listed in Table 4.1 while the background template was obtained using a data-driven technique in which one of the isolation variables used to select photon candidates is inverted. The data-driven technique, known as Side-Band-Subtraction (SBS), inverts the hollow cone track isolation $\left(\mathrm{IsO}_{T R K}\right)$ cut such that candidates in data with $2.0+0.006 * p_{T}<\mathrm{IsO}_{T R K}<$ $5 \mathrm{GeV}$ are selected for the background shape. The motivation behind using the SBS technique is the difference in behavior between photons and jets; a photon's shower shape is expected to be smaller than a jet's (as shown in Fig. 5.1). For the tracking 

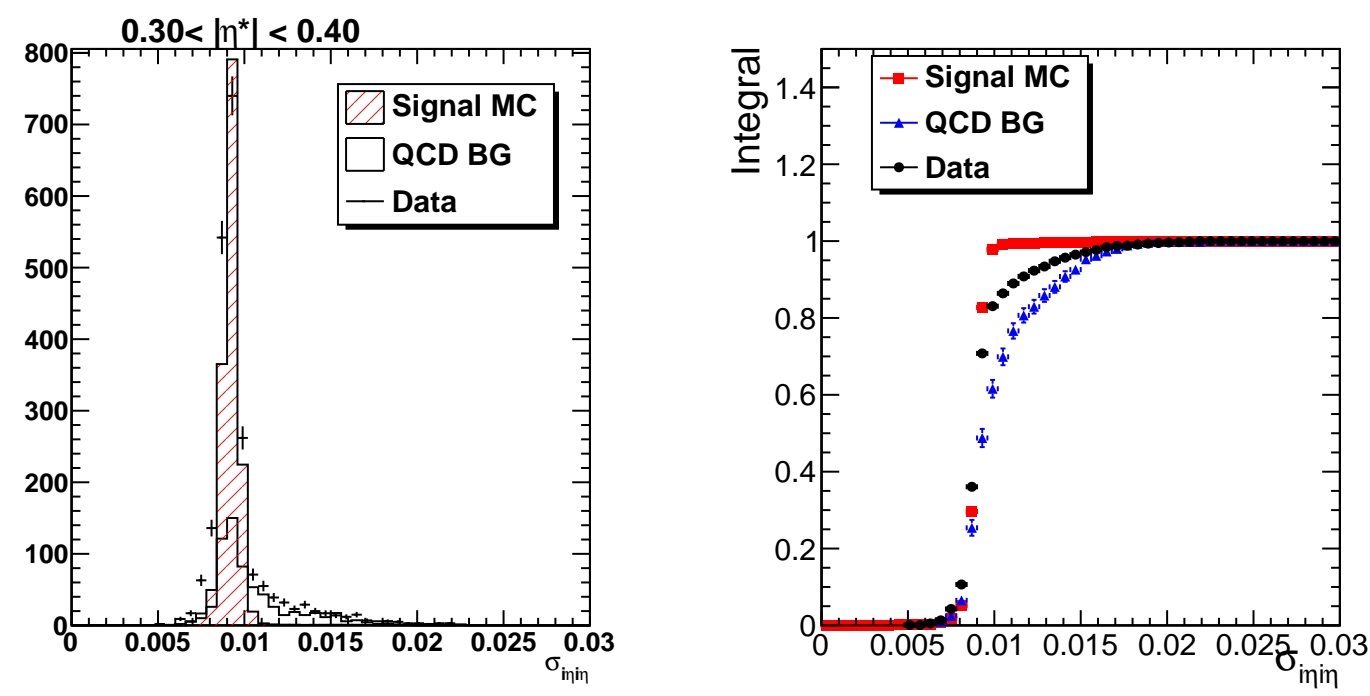

Figure 5.1: Signal (striped histogram) and background (white histogram) templates with data overlaid (crosses) for $\sigma_{i \eta i \eta}$, the discriminant variable with $0.30<\left|\eta^{*}\right|<0.40$. Bottom shows the area under the curve of the top plot is calculated for every point in $\sigma_{i \eta i \eta}$ for signal MC (squares), background (triangles) and data (circles).

system, this corresponds to having less energy deposits within the annulus cone used to measure $I s o_{T R K}$ for the photon candidate while having a larger energy deposit around the jet.

In order to validate that this data-driven technique models the expected background, the template shapes between QCD MC with photon identification cuts and SBS cuts were compared to data selected with SBS cuts for each bin in $\left|\eta^{*}\right|$. Fig. 5.2 shows the $\sigma_{i \eta i \eta}$ distribution (left) along with the area under that curve (right) for each set of cuts to data with SBS cuts. The $\sigma_{i \eta i \eta}$ distributions between the three background templates agree with each other. Moreover, the integral of these distributions are in good agreement, which further solidifies that data selected in the side-band region are comparable to using QCD MC as the background template for this study. Appendix D has additional plots showing other bins. 

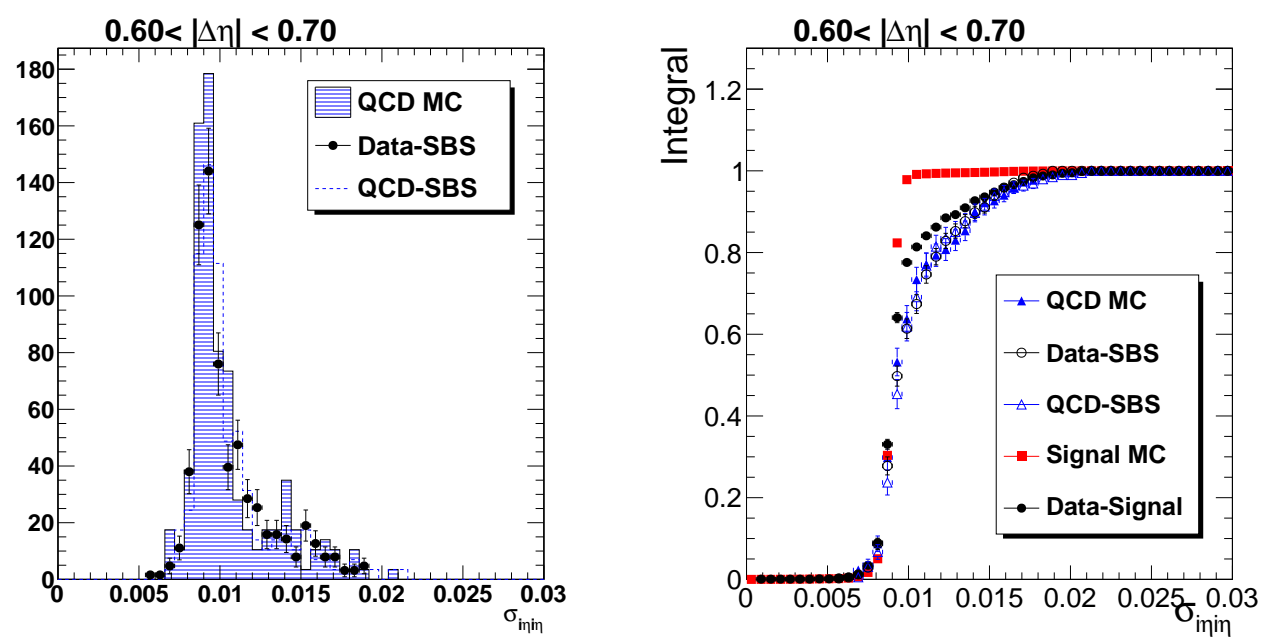

Figure 5.2: The $\sigma_{i \eta i \eta}$ template distribution for QCD MC with photon Identification cuts (striped histogram), QCD MC with SBS cuts (dotted line) and data with SBS cuts (circles) on the right and the area under the $\sigma_{i \eta i \eta}$ distribution for QCD MC with photon Identification cuts (closed triangles), QCD MC with SBS cut (open triangles) and Data SBS (open circles) on the right. Directly below the area plot is the ratio between QCD MC with photon Identification cuts to Data with SBS cuts (closed triangles) and the ratio of QCD MC with SBS cuts to data with SBS cuts (open triangles).

A 2-dimensional distribution of $\sigma_{i \eta i \eta}$ and $\mathrm{Iso}_{T R K}$ is shown in Fig. 5.3 to assist in giving a better visualization of this technique. The 2-D plane is divided into four regions containing A, B, C and D events. The horizontal line represents the boundary that is used to separate photon candidates selected in data with $\operatorname{Iso}_{T R K}<2.0+0.001^{*} p_{T}$ and the candidates in data used for the background template with SBS (note that candidates beyond $\mathrm{Iso}_{T R K}>5 \mathrm{GeV}$ are not selected for the background template). The horizontal line represents the boundary in $\sigma_{i \eta i \eta}$ that is used to separate photon background candidates that were selected using the standard photon identification cuts. 


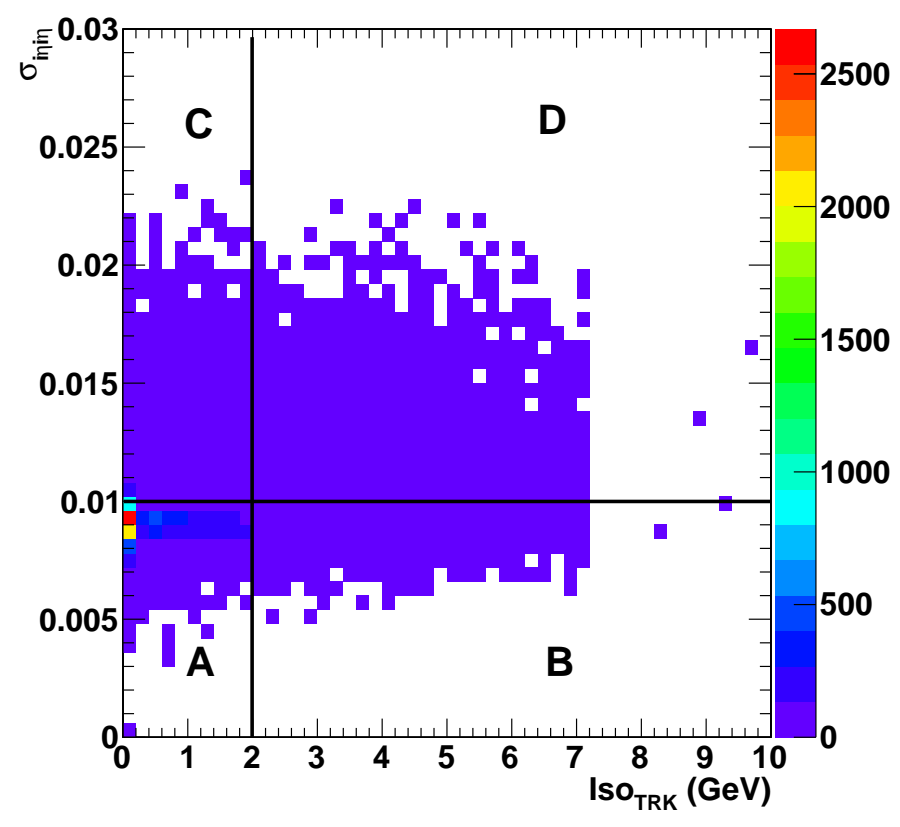

Figure 5.3: The $\sigma_{i \eta i \eta}$ Vs. Iso $T R K$ 2-dimensional distribution for 2010 data. The plane is divided into four regions labeled $\mathrm{A}, \mathrm{B}, \mathrm{C}$ and $\mathrm{D}$.

\subsection{Purity}

The purity in this analysis is determined using the two-bin expression:

$$
P=\frac{N_{\gamma}}{N}=\frac{f_{d a t a}-f_{b k g}}{f_{\text {sig }}-f_{b k g}}
$$

The fractions $f_{\text {data }}, f_{\text {sig }}$ and $f_{b k g}$ are defined as the fraction of candidates in the data, signal and background samples that are below the bin boundary of the discriminant distribution. The method divides the discriminating variable distribution at a point where the difference between the signal and background distribution is largest. The bin boundary location was obtained through plotting the area under the curve of the $\sigma_{i \eta i \eta}$ distribution at each point shown in Fig. 5.1, the location of the boundary was determined to be $\sigma_{i \eta i \eta}=0.01$; the location where the difference in integrals between the signal and background distributions is largest. The distribution in Fig 5.4 shows 
the fraction of candidates in signal, background and data as a function of $\left|\eta^{*}\right|$. The fraction errors were calculated using the binomial uncertainty expression:

$$
\Delta f=\sqrt{\frac{f(1-f)}{N_{\text {entries }}}}
$$

where $f$ corresponds to either $f_{\text {data }}, f_{\text {sig }}$ or $f_{b k g}$ and $N_{\text {entries }}$ is the number of entries in the $\sigma_{i \eta i \eta}$ for that particular $\left|\eta^{*}\right|$ bin.

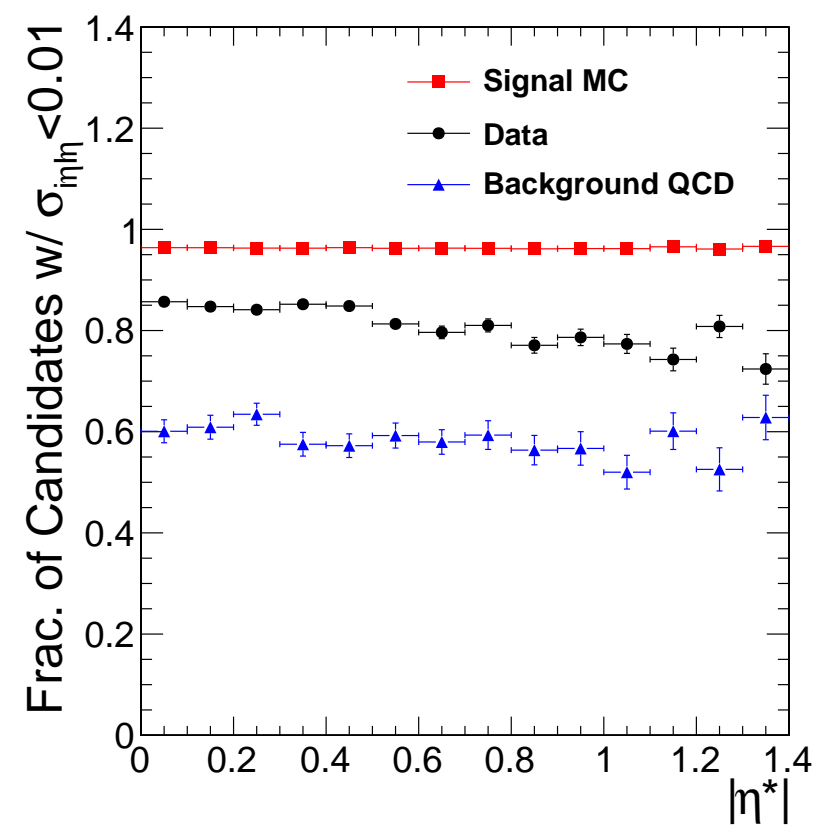

Figure 5.4: Fraction of candidates in the signal, background and data samples with $\sigma_{i \eta i \eta}$ $<0.01$ as a function of $\left|\eta^{*}\right|$.

The purity is calculated using Eq. 5.2 and shown in Fig. 5.5 and numerically in Table 5.2. The purity uncertainty is calculated using the following expression:

$$
\left(\frac{\Delta P}{P}\right)=\left(\frac{d P}{d f_{\text {sig }}} \Delta f_{\text {sig }}\right) \oplus\left(\frac{d P}{d f_{b k g}} \Delta f_{b k g}\right) \oplus\left(\frac{d P}{d f_{d a t}} \Delta f_{d a t}\right)
$$

where $\Delta f_{\text {sig }}, \Delta f_{b k g}$ and $\Delta f_{d a t}$ are the uncertainties reported in Table 5.1. 
Table 5.1: Fraction of candidates below $\sigma_{i \eta i \eta}=0.01$ for data, signal and background along with their uncertainties.

\begin{tabular}{|c|c|c|c|c|c|c|}
\hline Bin & $f_{\text {dat }}$ & $\Delta f_{\text {dat }}$ & $f_{\text {sig }}$ & $\Delta f_{\text {sig }}$ & $f_{\text {bkg }}$ & $\Delta f_{\text {bkg }}$ \\
\hline 0.05 & 0.8569 & $8.295 \times 10^{-3}$ & 0.9638 & $2.985 \times 10^{-4}$ & 0.6009 & $2.269 \times 10^{-2}$ \\
0.15 & 0.8473 & $8.802 \times 10^{-3}$ & 0.9637 & $3.071 \times 10^{-4}$ & 0.6089 & $2.362 \times 10^{-2}$ \\
0.25 & 0.8411 & $9.018 \times 10^{-3}$ & 0.9630 & $3.273 \times 10^{-4}$ & 0.6345 & $2.182 \times 10^{-2}$ \\
0.35 & 0.8520 & $9.087 \times 10^{-3}$ & 0.9628 & $3.546 \times 10^{-4}$ & 0.5753 & $2.343 \times 10^{-2}$ \\
0.45 & 0.8485 & $9.702 \times 10^{-3}$ & 0.9638 & $3.893 \times 10^{-4}$ & 0.5724 & $2.335 \times 10^{-2}$ \\
0.55 & 0.8131 & $1.129 \times 10^{-2}$ & 0.9626 & $4.515 \times 10^{-4}$ & 0.5924 & $2.472 \times 10^{-2}$ \\
0.65 & 0.7964 & $1.245 \times 10^{-2}$ & 0.9629 & $5.273 \times 10^{-4}$ & 0.5797 & $2.426 \times 10^{-2}$ \\
0.75 & 0.8101 & $1.288 \times 10^{-2}$ & 0.9625 & $6.348 \times 10^{-4}$ & 0.5933 & $2.836 \times 10^{-2}$ \\
0.85 & 0.7708 & $1.552 \times 10^{-2}$ & 0.9615 & $7.844 \times 10^{-4}$ & 0.5636 & $2.907 \times 10^{-2}$ \\
0.95 & 0.7865 & $1.624 \times 10^{-2}$ & 0.9620 & $9.641 \times 10^{-4}$ & 0.5670 & $3.311 \times 10^{-2}$ \\
1.05 & 0.7735 & $1.874 \times 10^{-2}$ & 0.9619 & $1.215 \times 10^{-3}$ & 0.5200 & $3.331 \times 10^{-2}$ \\
1.15 & 0.7428 & $2.239 \times 10^{-2}$ & 0.9655 & $1.477 \times 10^{-3}$ & 0.6011 & $3.620 \times 10^{-2}$ \\
1.25 & 0.8081 & $2.191 \times 10^{-2}$ & 0.9611 & $2.042 \times 10^{-3}$ & 0.5255 & $4.266 \times 10^{-2}$ \\
1.35 & 0.7240 & $3.007 \times 10^{-2}$ & 0.9664 & $2.488 \times 10^{-3}$ & 0.6281 & $4.394 \times 10^{-2}$ \\
\hline
\end{tabular}

Appendix E discusses in detail how the purity technique used in this analysis is equivalent to what is known as the $\mathrm{ABCD}$ method with was visually shown in the Fig. 5.3 distribution.

The reliability of the purity calculations were determined using pull distributions. Pseudo experiments were generated using the number of candidates in data, the purity and MC templates for both signal and background for each $\left|\eta^{*}\right|$ bin as follows: The number of candidates in data, $N_{d a t a}$, was used as the seed to generate a random number, $N_{\text {data }}^{\text {rand }}$. The number of candidates along with the purity, $P$, determined in each $\left|\eta^{*}\right|$ bin was then used to obtain the number of candidates that contributed to signal and background using the following expression:

$$
\begin{gathered}
N_{\text {signal }}^{\text {rand }}=N_{\text {data }}^{\text {rand }} * P \\
N_{b k g}^{\text {rand }}=N_{\text {data }}^{\text {rand }}(1-P)
\end{gathered}
$$


Table 5.2: Purity and uncertainty for each $\left|\eta^{*}\right|$.

\begin{tabular}{|c|c|c|}
\hline Bin & Purity & $\Delta P$ \\
\hline 0.05 & 0.7055 & $2.935 \times 10^{-2}$ \\
0.15 & 0.6719 & $3.305 \times 10^{-2}$ \\
0.25 & 0.6291 & $3.690 \times 10^{-2}$ \\
0.35 & 0.7140 & $2.914 \times 10^{-2}$ \\
0.45 & 0.7053 & $3.039 \times 10^{-2}$ \\
0.55 & 0.5961 & $4.072 \times 10^{-2}$ \\
0.65 & 0.5654 & $4.259 \times 10^{-2}$ \\
0.75 & 0.5872 & $4.716 \times 10^{-2}$ \\
0.85 & 0.5207 & $5.243 \times 10^{-2}$ \\
0.95 & 0.5557 & $5.547 \times 10^{-2}$ \\
1.05 & 0.5738 & $5.322 \times 10^{-2}$ \\
1.15 & 0.3888 & $8.639 \times 10^{-2}$ \\
1.25 & 0.6485 & $6.103 \times 10^{-2}$ \\
1.35 & 0.2834 & 0.1287 \\
\hline
\end{tabular}

The number of signal and background candidates in each $\left|\eta^{*}\right|$ bin were then used to randomize the signal and background templates obtained in Monte Carlo. The randomized distributions comprise the pseudo data distribution and the purity is then calculated along with the uncertainty using Eqs. 5.2 and 5.4 , respectively. The purity, $P_{\text {pseudo }}$, and uncertainty, $\Delta P_{\text {pseudo }}$, calculated is then used to measure the pull:

$$
\text { pull }=\frac{P_{\text {pseudo }}-P}{\Delta P_{\text {pseudo }}}
$$

In total, 1000 pseudo experiments were generated to measure the purity pulls. If the uncertainty is reliable then the pull distribution will have $\mu=0$ (mean) with a $\sigma=1$ (width) in value. The pull distribution shown in Fig. 5.6 is for toy experiment candidates with $0.4<\left|\eta^{*}\right|<0.5$ and is fitted with a Gaussian function. The mean reports a value of $0.02125 \pm 0.02404$, which is consistent with the expected mean. The $\sigma$ of the distribution however reports a value of $0.7423 \pm 0.0180$, which is less 
than the expected value for the width. The low value in the width indicates that the purity uncertainty is overestimated. The purity uncertainty can be adjusted by multiplying it by the reported width value reported for each $\left|\eta^{*}\right|$ bin. Table 5.3 shows the width reported as well as the corrected purity uncertainty, $\Delta P_{\text {corr }}$, for each bin in $\left|\eta^{*}\right|$. Appendix F shows the pull distributions for each $\left|\eta^{*}\right|$ bin.

Table 5.3: The purity uncertainty corrected, $\Delta P_{\text {corr }}$, using the correction factor, $\sigma$, reported by the pull distributions shown in Appendix $\mathrm{F}$

\begin{tabular}{|c|c|c|}
\hline Bin & $\sigma$ & $\Delta P_{\text {corr }}$ \\
\hline 0.05 & 0.75 & $2.201 \times 10^{-2}$ \\
0.15 & 0.71 & $2.347 \times 10^{-2}$ \\
0.25 & 0.71 & $2.620 \times 10^{-2}$ \\
0.35 & 0.74 & $2.156 \times 10^{-2}$ \\
0.45 & 0.74 & $2.249 \times 10^{-2}$ \\
0.55 & 0.69 & $2.810 \times 10^{-2}$ \\
0.65 & 0.67 & $2.854 \times 10^{-2}$ \\
0.75 & 0.70 & $3.301 \times 10^{-2}$ \\
0.85 & 0.67 & $3.513 \times 10^{-2]}$ \\
0.95 & 0.32 & $1.775 \times 10^{-2}$ \\
1.05 & 0.35 & $1.863 \times 10^{-2}$ \\
1.15 & 0.28 & $2.419 \times 10^{-2}$ \\
1.25 & 0.39 & $2.380 \times 10^{-2}$ \\
1.35 & 0.28 & $3.604 \times 10^{-2}$ \\
\hline
\end{tabular}




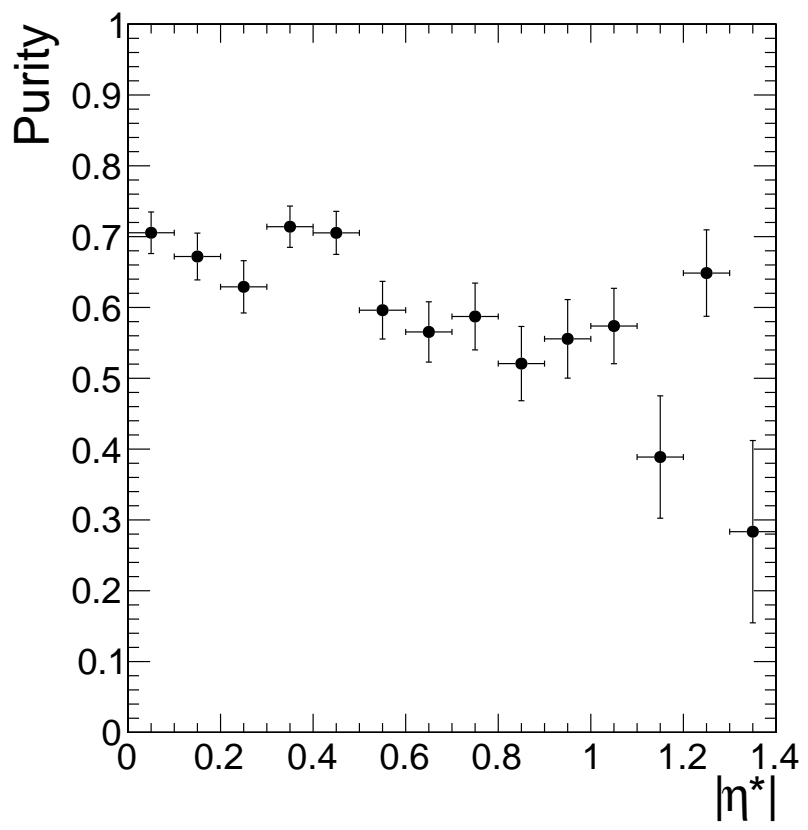

Figure 5.5: Purity as a function of $\left|\eta^{*}\right|$. The uncertainties shown are calculated using Eq. 5.4 .

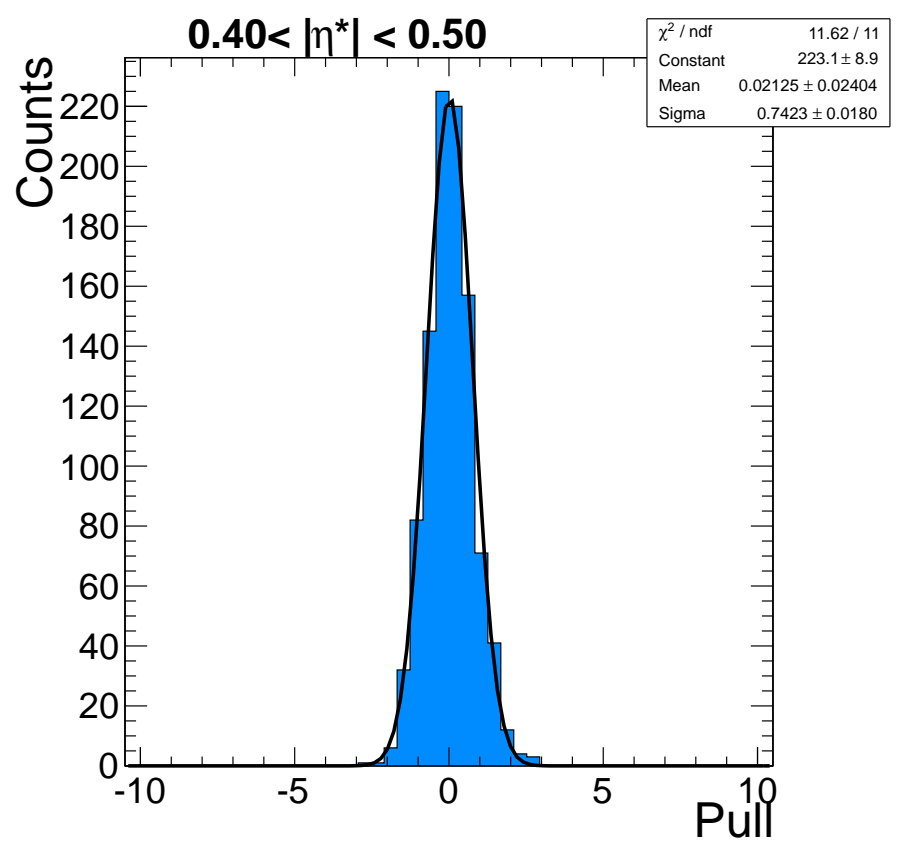

Figure 5.6: Pull distribution for candidates from the pseudo experiments generated with $0.4<\left|\eta^{*}\right|<0.5$ fitted with a Gaussian function. The mean for the pull distribution is centered at 0 while the width $(\sigma)$ is less than 1 . 


\section{CHAPTER 6}

\section{SYSTEMATIC UNCERTAINTIES}

The main contributors to the systematic uncertainties are the background template obtained using the data-driven technique and the $\eta$ positioning resolution of the jet. Each contribution is discussed in detail.

\subsection{Background Shape}
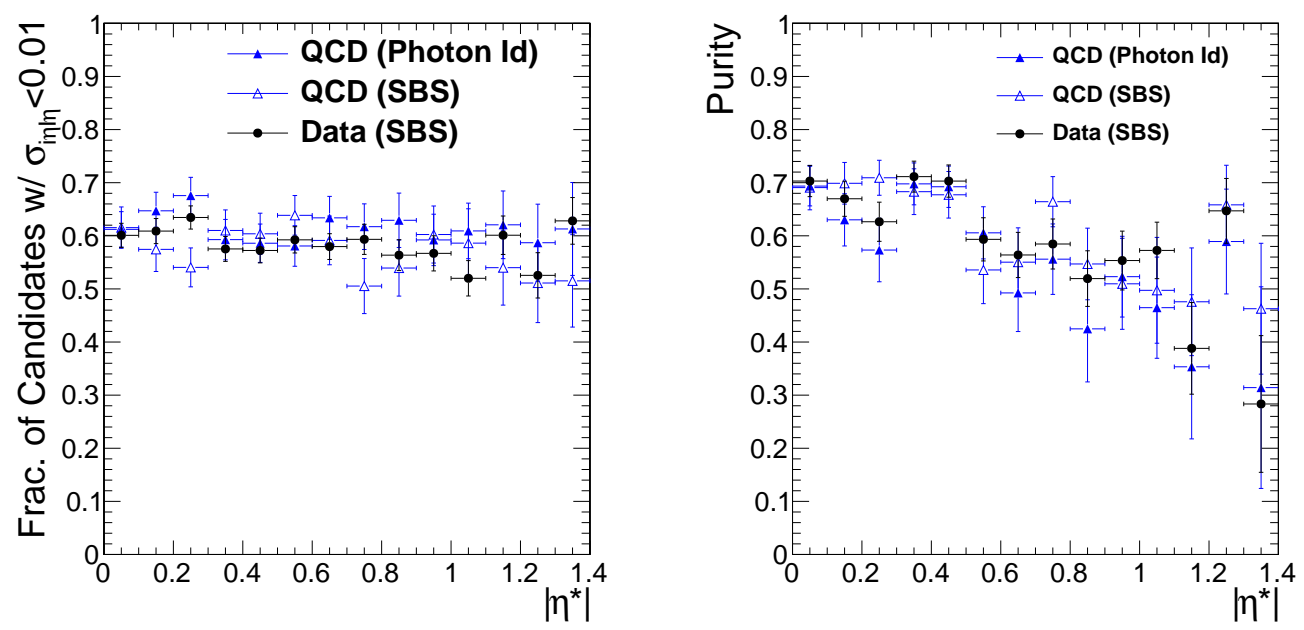

Figure 6.1: The fraction of candidates below $\sigma_{i \eta i \eta}=0.01$ for QCD MC with photon identification cuts (solid triangles), QCD MC selected with SBS cuts (open triangles) and data selected with SBS cuts (solid circles) on the left. The purity as a function of $\left|\eta^{*}\right|$ for QCD MC with photon identification cuts (solid triangles), QCD MC selected with SBS cuts (open triangles) and Data selected with SBS cuts (solid circles) on the right. Both plots give similar results for each background template.

It was shown in Chapter 5 that the data driven background template is comparable with the QCD MC background template, but there are some differences in their final purity result that will be taken into account as the systematic uncertainty on the background shape. The left hand plot in Fig. 6.1 shows that the fraction of candidates in the data driven background template with $\sigma_{i \eta i}<0.01$ agrees within uncertainty with the QCD MC background templates (one selected using photon identification cuts, the other selected in the side-band region) and the right had plot in the same 
figure shows the resulting purity calculated using the different background templates. It is these differences that will be used to calculate the systematic uncertainty of the background shape.

The systematic uncertainty associated with the background shape is calculated as follows: The $\left|\eta^{*}\right|$ distribution is plotted using the QCD MC background template obtained with the inverted $\mathrm{Iso}_{T R K}$, as was done to obtain the data-driven background template. The difference between the two distributions is taken as the systematic uncertainty due to the background shape:

$$
\Delta_{\text {bkgshape }}=\frac{\left|Q C D_{s b s}-Q C D_{\text {pho }}\right|}{Q C D_{s b s}}
$$

The uncertainties range between $0.4-10.7 \%$, a list of the systematic uncertainties due to the background shape are shown in Table 6.3. The background template beyond $\left|\eta^{*}\right|=1.4$ can not be validated due to a lack of statistics in the $\sigma_{i \eta i \eta}$ distributions.

Table 6.1: Fraction of candidates with $\sigma_{i \eta i \eta}<0.01$ for QCD MC candidates selected using photon identification cuts, QCD MC candidates selected using sbs cuts and data selected using sbs cuts along with their respective uncertainty.

\begin{tabular}{|c|c|c|c|c|c|c|}
\hline Bin & $f_{b k g Q C D_{p h o}}$ & $\Delta f_{b k g Q C D_{p h o}}$ & $f_{b k g Q C D_{s b s}}$ & $\Delta f_{b k g Q C D_{s b s}}$ & $f_{b k g D a t a}{ }_{s b s}$ & $\Delta f_{b k g D a t a} a_{s b s}$ \\
\hline 0.05 & 0.6122 & $3.331 \times 10^{-2}$ & 0.6154 & $3.895 \times 10^{-2}$ & 0.6009 & $2.269 \times 10^{-2}$ \\
\hline 0.15 & 0.6471 & $3.350 \times 10^{-2}$ & 0.5745 & $4.164 \times 10^{-2}$ & 0.6089 & $2.362 \times 10^{-2}$ \\
\hline 0.25 & 0.6757 & $3.344 \times 10^{-2}$ & 0.5405 & $3.664 \times 10^{-2}$ & 0.6345 & $2.182 \times 10^{-2}$ \\
\hline 0.35 & 0.5928 & $3.802 \times 10^{-2}$ & 0.6101 & $3.868 \times 10^{-2}$ & 0.5753 & $2.343 \times 10^{-2}$ \\
\hline 0.45 & 0.5860 & $3.612 \times 10^{-2}$ & 0.6038 & $3.879 \times 10^{-2}$ & 0.5724 & $2.335 \times 10^{-2}$ \\
\hline 0.55 & 0.5808 & $3.812 \times 10^{-2}$ & 0.6386 & $3.728 \times 10^{-2}$ & 0.5924 & $2.472 \times 10^{-2}$ \\
\hline 0.65 & 0.63 & $4.043 \times 10^{-2}$ & 0.5913 & $4.584 \times 10^{-2}$ & 797 & $2.426 \times 10^{-2}$ \\
\hline 0.75 & 0.6172 & $4.296 \times 10^{-2}$ & 0.5054 & $5.184 \times 10^{-2}$ & 0.5933 & $2.836 \times 10^{-2}$ \\
\hline 0.85 & 0.6292 & $5.120 \times 10^{-2}$ & 0.5393 & $5.284 \times 10^{-2}$ & 0.5638 & $2.907 \times 10^{-2}$ \\
\hline 0.95 & 0.5922 & $4.842 \times 10^{-2}$ & 0.6024 & $5.372 \times 10^{-2}$ & 0.5670 & $3.311 \times 10^{-2}$ \\
\hline 1.05 & 0.6092 & $5.231 \times 10^{-2}$ & 0.5862 & $6.467 \times 10^{-2}$ & 0.5200 & $3.331 \times 10^{-2}$ \\
\hline 1.15 & 0.6207 & $6.371 \times 10^{-2}$ & 0.5400 & $7.048 \times 10^{-2}$ & 0.6011 & $3.620 \times 10^{-2}$ \\
\hline 1.25 & 0.5870 & $7.260 \times 10^{-2}$ & 0.5111 & $7.452 \times 10^{-2}$ & 0.5255 & $4.266 \times 10^{-2}$ \\
\hline 1.35 & 0.6129 & $8.748 \times 10^{-2}$ & 0.5152 & $8.700 \times 10^{-2}$ & 0.6281 & $4.394 \times 10^{-2}$ \\
\hline
\end{tabular}


Table 6.2: Purities calculated using three background templates obtained from QCD MC candidates selected with photon identification cuts, QCD MC candidates selected with sbs cuts and data selected with sbs cuts along with their respective uncertainty.

\begin{tabular}{|c|c|c|c|c|c|c|}
\hline Bin & $P_{Q C D_{\text {pho }}}$ & $\Delta P_{Q C D_{\text {pho }}}$ & $\mathrm{P}_{Q C D_{s b s}}$ & $\Delta P_{Q C D_{s b s}}$ & $\mathrm{P}_{\text {Data }_{s b s}}$ & $\Delta P_{\text {Data }_{s b s}}$ \\
\hline 0.05 & 0.6936 & $3.728 \times 10^{-2}$ & 0.6908 & $4.183 \times 10^{-2}$ & 0.7031 & $2.935 \times 10^{-2}$ \\
0.15 & 0.6301 & $4.921 \times 10^{-2}$ & 0.6989 & $3.924 \times 10^{-2}$ & 0.6697 & $3.304 \times 10^{-2}$ \\
0.25 & 0.5732 & $5.971 \times 10^{-2}$ & 0.7093 & $3.294 \times 10^{-2}$ & 0.6265 & $3.686 \times 10^{-2}$ \\
0.35 & 0.6978 & $3.944 \times 10^{-2}$ & 0.6831 & $4.309 \times 10^{-2}$ & 0.7114 & $2.913 \times 10^{-2}$ \\
0.45 & 0.6923 & $3.892 \times 10^{-2}$ & 0.6772 & $4.384 \times 10^{-2}$ & 0.7030 & $3.037 \times 10^{-2}$ \\
0.55 & 0.6056 & $4.902 \times 10^{-2}$ & 0.5357 & $6.344 \times 10^{-2}$ & 0.5933 & $4.065 \times 10^{-2}$ \\
0.65 & 0.4945 & $7.271 \times 10^{-2}$ & 0.5504 & $6.463 \times 10^{-2}$ & 0.5639 & $4.253 \times 10^{-2}$ \\
0.75 & 0.5560 & $6.633 \times 10^{-2}$ & 0.6642 & $4.721 \times 10^{-2}$ & 0.5846 & $4.708 \times 10^{-2}$ \\
0.85 & 0.4248 & $9.989 \times 10^{-2}$ & 0.5470 & $6.742 \times 10^{-2}$ & 0.5194 & $5.236 \times 10^{-2}$ \\
0.95 & 0.5231 & $7.602 \times 10^{-2}$ & 0.5096 & $8.567 \times 10^{-2}$ & 0.5534 & $5.537 \times 10^{-2}$ \\
1.05 & 0.4647 & $9.528 \times 10^{-2}$ & 0.4974 & $9.962 \times 10^{-2}$ & 0.5725 & $5.316 \times 10^{-2}$ \\
1.15 & 0.3534 & 0.1357 & 0.4758 & 0.1014 & 0.388074 & $8.628 \times 10^{-2}$ \\
1.25 & 0.5892 & $9.868 \times 10^{-2}$ & 0.6583 & $7.454 \times 10^{-2}$ & 0.6470 & $6.097 \times 10^{-2}$ \\
1.35 & 0.3142 & 0.1898 & 0.4627 & 0.123181 & 0.2834 & 0.1287 \\
\hline
\end{tabular}

\subsection{Jet $\eta$ Position Resolution}

The angular distribution depends on how accurate the $\eta$ positions are for the photon and jet candidates in data. However, as a result of limitations in resolution and detector acceptance these quantities may be distorted. In order to obtain the original (true) $\eta$ distributions the use of unfolding techniques are applied. It was found (see Appendix G) that unfolding $\left|\eta^{*}\right|$ varies the distribution by i $4 \%$ in the highest $\left|\eta^{*}\right|$ bin.

The systematic uncertainty contribution from the jet's $\eta$ resolution was calculated in the following way: the $\eta^{\text {jet }}$ was smeared using a Gaussian random number distribution where $\sigma$ was taken as:

$$
\sigma=A+\frac{B}{\sqrt{E_{T}^{j e t}}}
$$


Table 6.3: Number of entries in $\left|\eta^{*}\right|$ using the background templates obtained from QCD MC along with the systematic uncertainty due to the background shape.

\begin{tabular}{|c|c|c|c|}
\hline Bin & $\mathrm{N}_{\text {corr }, Q C D_{\text {phoI }}}$ & $\mathrm{N}_{\text {corr }, Q C D_{\text {sbs }}}$ & $\Delta_{\text {bkgshape }}$ \\
\hline 0.05 & 0.138252 & 0.144823 & 4.753 \\
0.15 & 0.124604 & 0.124019 & 0.4692 \\
0.25 & 0.125679 & 0.117194 & 6.751 \\
0.35 & 0.116634 & 0.123452 & 5.846 \\
0.45 & 0.104205 & 0.105447 & 1.191 \\
0.55 & 0.073639 & 0.0813946 & 10.53 \\
0.65 & 0.0656527 & 0.0625033 & 4.797 \\
0.75 & 0.0661328 & 0.0629075 & 4.877 \\
0.85 & 0.0418677 & 0.0395392 & 5.561 \\
0.95 & 0.0423814 & 0.0393583 & 7.133 \\
1.05 & 0.0300357 & 0.0319578 & 6.399 \\
1.15 & 0.0193952 & 0.0176985 & 8.748 \\
1.25 & 0.0235877 & 0.0210464 & 10.77 \\
1.35 & 0.0102189 & 0.00934117 & 8.589 \\
\hline
\end{tabular}

where $A=0.01$ and $B=0.36$. With $\eta^{\text {jet }}$ smeared, $\left|\eta^{*}\right|$ was re-plotted and the ratio was taken between the original distribution and the smeared distribution. The distribution shown in Fig. 6.3 are the raw distributions for $\left|\eta^{*}\right|$ with the original $\eta^{\text {jet }}$ and the smeared $\eta^{\text {jet }}$ values.

The ratio distribution directly beneath the $\left|\eta^{*}\right|$ distributions shows that the difference between the original and smeared distributions is $<4 \%$. The systematic uncertainty due to the jet $\eta$ resolution is taken as the uncertainty of the weighted average of the ratio points:

$$
\Delta_{\eta_{j e t} \text { res }}=\sqrt{\frac{\sum_{i}\left(R_{i}-1.0\right)^{2} \frac{1}{\Delta R_{i}}}{\sum_{i} \frac{1}{\Delta R_{i}}}}
$$

where $R_{i}$ is the ratio between the original and smeared $\left|\eta^{*}\right|$ value for the $i^{\text {th }}$ bin and $\Delta R_{i}$ is the uncertainty of $R_{i}$. Eq. 6.3 reports an uncertainty of $2.258 \%$ due to the jet $\eta$ resolution. Table 6.4 lists the systematic uncertainties for this analysis. 
Table 6.4: Systematic uncertainties for this analysis.

\begin{tabular}{|c|c|}
\hline Contribution & Uncertainty (\%) \\
\hline Background shape & $0.4-10.7 \%$ \\
Jet $\eta$ position resolution & $2.258 \%$ \\
\hline
\end{tabular}

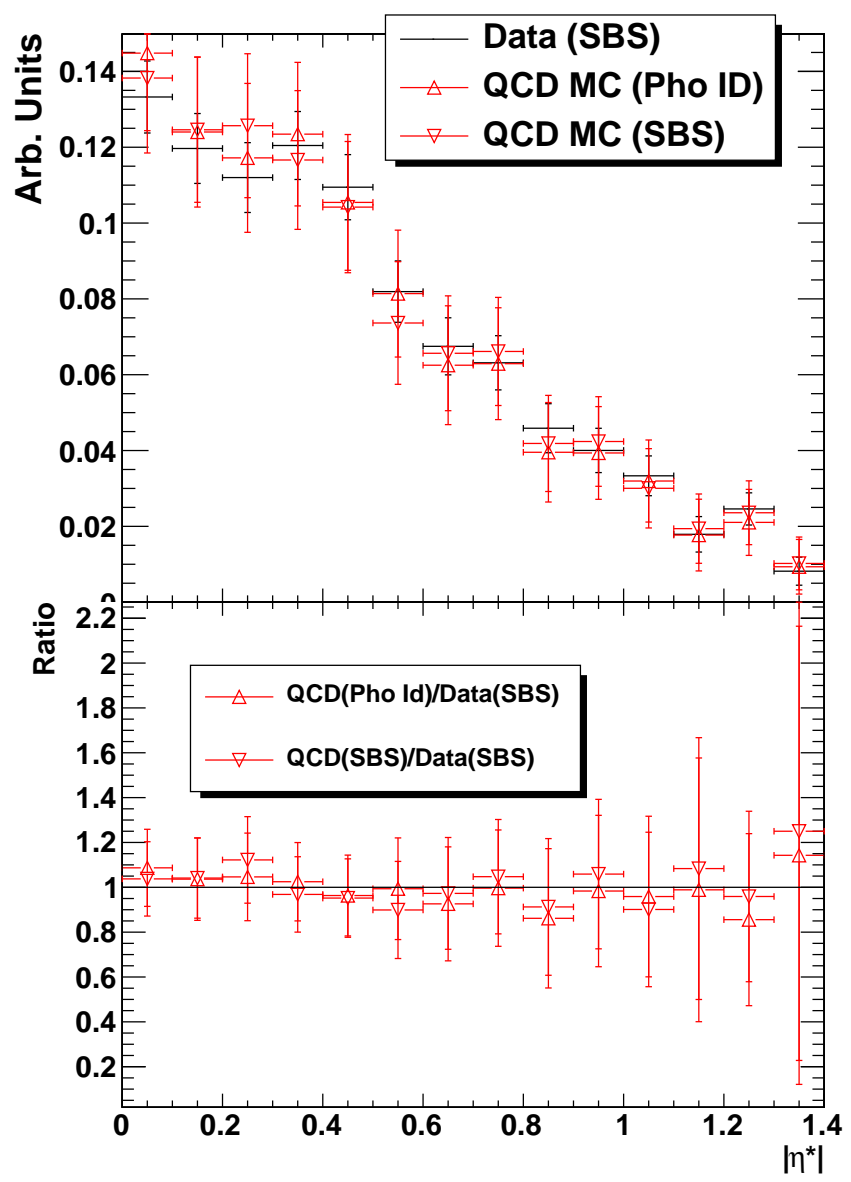

Figure 6.2: Plot showing $\left|\eta^{*}\right|$ using two different background templates; Data selected with SBS cuts and QCD MC selected with photon identification cuts on top. The bottom plot is the ratio between the two results, taken as the systematic uncertainty of the background shape. The largest deviation between data and both set of cuts used on QCD is seen in the highest $\left|\eta^{*}\right|$ bin. 


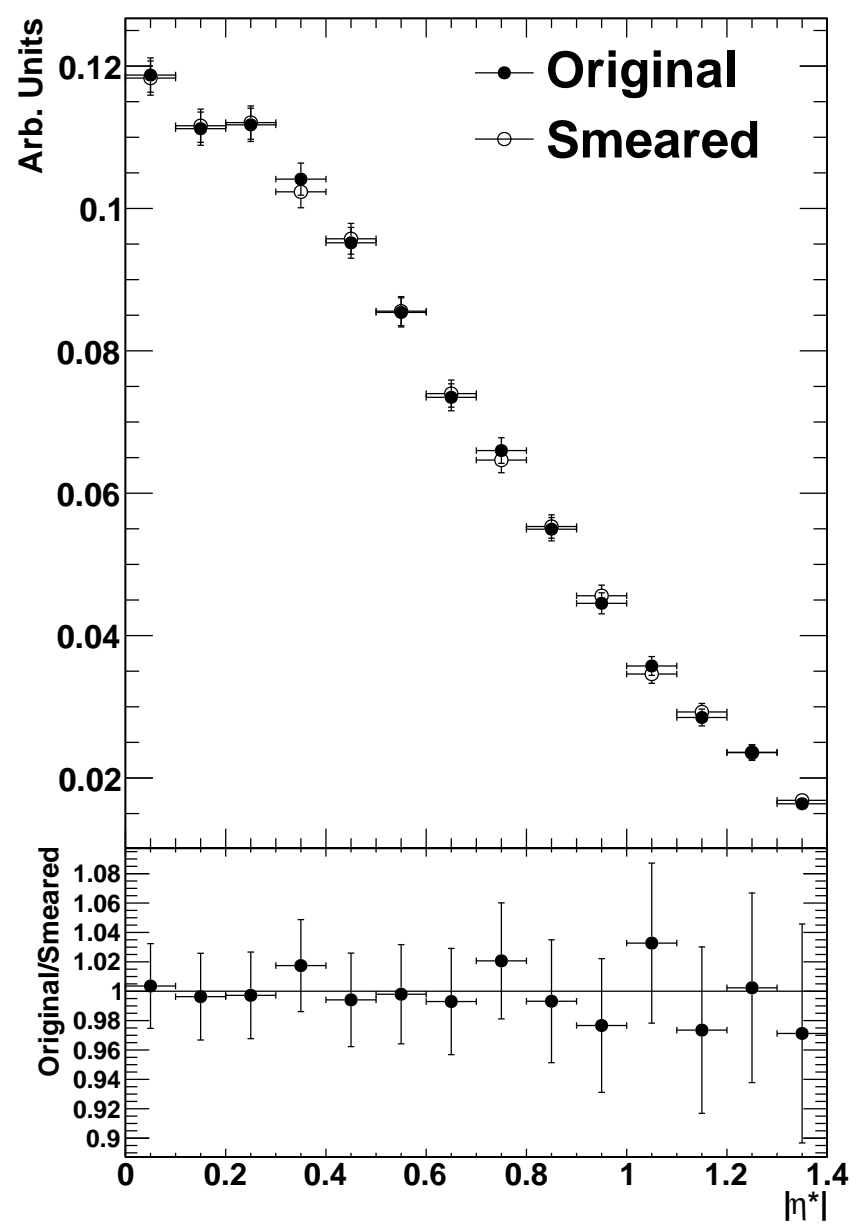

Figure 6.3: Angular distribution $\left|\eta^{*}\right|$ using the original $\eta^{\text {jet }}$ in black solid circles and the smeared $\eta^{\text {jet }}$ in the black open circles. Below is the ratio between the two distributions showing that the jet resolution does not affect the $\left|\eta^{*}\right|$ distribution by much. 


\section{CHAPTER 7}

\section{THE ANGULAR DISTRIBUTION}

The angular distribution is expressed as:

$$
\frac{d \sigma}{d\left|\eta^{*}\right|}=\frac{N \times P}{\epsilon \int \mathcal{L} d t\left|\Delta \eta^{*}\right|}
$$

where $N$ is the number of events, $P$ is the purity, $\epsilon$ is the efficiency, $\left|\Delta \eta^{*}\right|$ is the bin width and $\int \mathcal{L} d t$ is the effective luminosity. Measuring the angular distribution is a complimentary analysis to the inclusive photon cross section measurement, but with the added benefit that the analysis is decoupled from parton distribution functions. The prompt photon angular distribution measurement presented is the first time this analysis is preformed at CMS as well as for the LHC experiments. It is also the first time in over a decade that such a test of NLO QCD is performed, with the last tests made at the Tevatron in the late $20^{\text {th }}$ century.

\subsection{NLO Theoretical Prediction}

The NLO QCD prediction for the angular distribution used is a program developed by H. Baer, J. Ohnemus and J. Owens. The theoretical prediction uses $2 \rightarrow 2$ prompt photon processes at leading order. At NLO, $2 \rightarrow 3$ and one loop contributions are produced [59]. The Feynman diagrams at LO and NLO were discussed in Sections 1.2.1 and 1.2 .2 and shown in Figs. 1.10 through 1.13 as well.

\subsubsection{Theoretical Uncertainty}

The theoretical uncertainty was calculated by varying the factorization and renormalization scales, $\mu_{F}$ and $\mu_{R}$ which are traditionally taken to be the same as the $p_{T}$ of the

photon candidate in the event. For consistency, $\mu_{F}$ and $\mu_{R}$ were varied by multiplies

of $p_{T}$ in two ways: The first variation is when $\mu_{F}=2^{*} p_{T}^{\gamma}$ and $\mu_{R}=0.5^{*} p_{T}^{\gamma}$, the second 


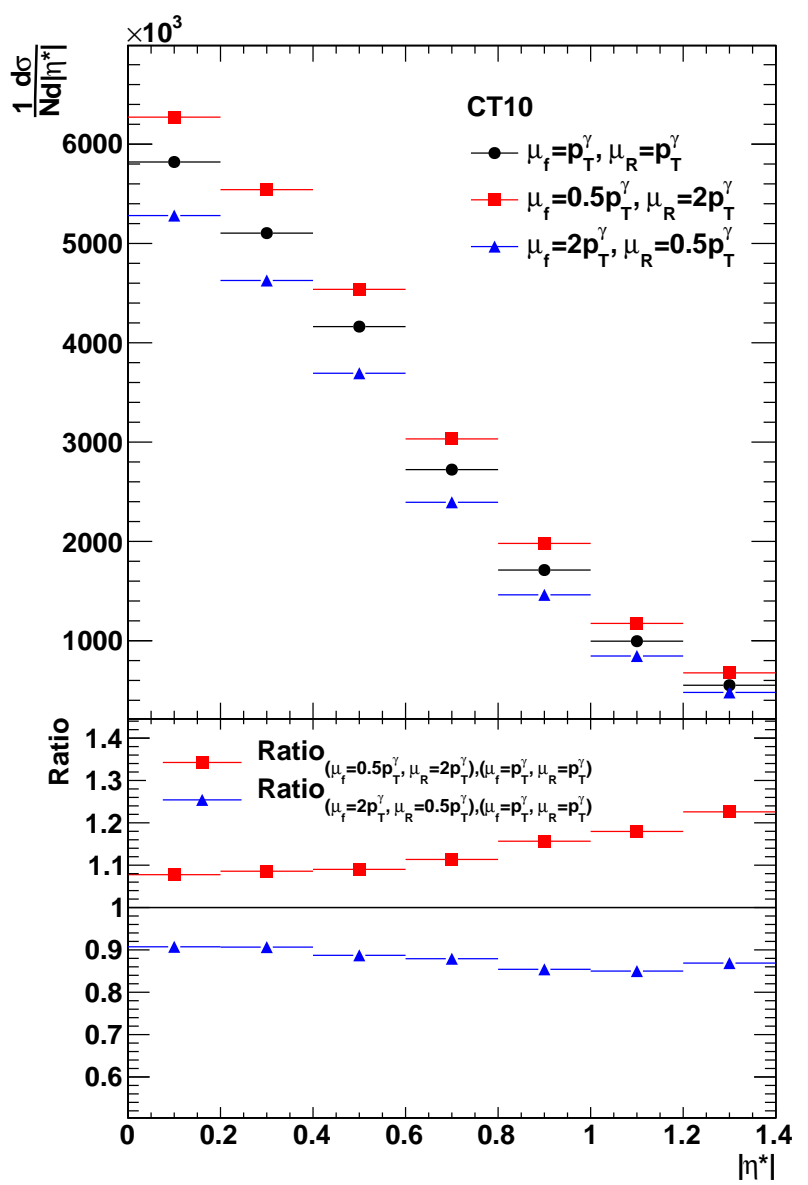

Figure 7.1: The top plot shows the angular distribution $\left|\eta^{*}\right|$ using three different sets of values for $\mu_{F}$ and $\mu_{F}$, which represent the factorization scale and renormalization factor, respectively. These values are traditionally taken to be equal to the $p_{T}$ of the photon. Below is the ratio between the varied and traditional values of the scales. These differences are taken as the theoretical uncertainty. 
variation is when $\mu_{F}=0.5^{*} p_{T}^{\gamma}$ and $\mu_{R}=2^{*} p_{T}^{\gamma}$. The distributions in Fig. 7.1 are the resulting raw angular distribution on top and the ratio between the scale variations to the nominal scaling values on the bottom of the figure. The nominal scaling values are traditionally taken as the $p_{T}$ of the photon candidate. The ratio shown in Fig. 7.1 is taken as the theoretical uncertainty. Table 7.1 shows the values of the theoretical uncertainty for this analysis.

Table 7.1: Theoretical uncertainty due to varying the factorization scale, $\mu_{F}$, and the renormalization scale, $\mu_{R}$.

\begin{tabular}{|c|c|}
\hline Bin & Uncertainty $(+-)$ \\
\hline $0.0-0.2$ & $+0.07758-0.09268$ \\
$0.2-0.4$ & $+0.08575-0.09343$ \\
$0.4-0.6$ & $+0.09010-0.1128$ \\
$0.6-0.8$ & $+0.1135-0.1208$ \\
$0.8-1.0$ & $+0.1566-0.1459$ \\
$1.0-1.2$ & $+0.1796-0.1500$ \\
$1.2-1.4$ & $+0.2259-0.1311$ \\
\hline
\end{tabular}

\subsection{Results}

The entire $5.56 \mathrm{fb}^{-1}$ of data recorded at CMS during the 2011 run year was analyzed, which roughly corresponds to about 106 million events. After selecting events based on the various filters applied to the analysis as well as the photon HLT path about $4.905 \mathrm{pb}^{-1}$ of data, about 20.7 million events, remained for the analysis. After photon identification cuts are applied there are $\sim 14.3 \mathrm{~K}$ events used to measure the angular distribution.

The uncertainty contributions for efficiency and purity for each $\left|\eta^{*}\right|$ bin were discussed and shown in Tables 4.6 and 5.2, respectively. The luminosity uncertainty is $4 \%$ [60]. The uncertainty on the number of candidates in each $\left|\eta^{*}\right|$ bin are dictated by Poission statistics $(\sqrt{N})[58$. The uncertainties due to the number of candidates, purity and efficiency were added in quadrature in order to obtain the full uncorrelated uncertainty: 


$$
\Delta_{\text {uncorr }}=\frac{\Delta N}{N} \oplus \frac{\Delta P}{P} \oplus \frac{\Delta \epsilon}{\epsilon}
$$

Table 7.2: Values for the number of candidates $(N)$, and the relative statistical uncertainties due to the number of candidates $\left(\frac{\Delta N}{N}\right)$, purity $\left(\frac{\Delta P}{P}\right)$ and efficiency $\left(\frac{\Delta \epsilon}{\epsilon}\right)$ for each bin in $\left|\eta^{*}\right|$. Also included are the total uncorrelated $\left(\Delta_{\text {uncorr }}\right)$ and correlated $\left(\Delta_{\text {corr }}\right)$ uncertainties.

\begin{tabular}{|c|c|c|c|c|c|c|c|}
\hline Bin & $N$ & $\frac{\Delta N}{N}$ & $\frac{\Delta P}{P}$ & $\frac{\Delta \epsilon}{\epsilon}$ & $N_{\text {corr }}$ & $\Delta_{\text {uncorr }}(\%)$ & $\Delta_{\text {corr }}(\%)$ \\
\hline 0.05 & 2429 & 0.02029 & 0.03118 & $5.588 \times 10^{-4}$ & 397.9 & 3.71234 & 6.610 \\
0.15 & 2275 & 0.02097 & 0.03455 & $5.751 \times 10^{-4}$ & 355.2 & 4.04183 & 4.617 \\
0.25 & 2286 & 0.02092 & 0.04090 & $6.067 \times 10^{-4}$ & 334.6 & 4.59392 & 8.166 \\
0.35 & 2130 & 0.02167 & 0.03081 & $6.595 \times 10^{-4}$ & 354.2 & 3.76679 & 7.434 \\
0.45 & 1947 & 0.02266 & 0.03271 & $7.323 \times 10^{-4}$ & 320.1 & 3.98013 & 4.745 \\
0.55 & 1747 & 0.02393 & 0.04747 & $8.308 \times 10^{-4}$ & 242.2 & 5.31626 & 11.49 \\
0.65 & 1503 & 0.02579 & 0.05413 & $9.924 \times 10^{-4}$ & 198.5 & 5.99653 & 6.642 \\
0.75 & 1350 & 0.02722 & 0.05608 & $1.178 \times 10^{-3}$ & 185.1 & 6.23441 & 6.699 \\
0.85 & 1124 & 0.02983 & 0.08154 & $1.423 \times 10^{-3}$ & 136.6 & 8.68391 & 7.213 \\
0.95 & 911 & 0.033132 & 0.03370 & $1.767 \times 10^{-3}$ & 119 & 4.72933 & 8.484 \\
1.05 & 731 & 0.036986 & 0.03113 & $2.19 \times 10^{-3}$ & 98.1 & 4.83901 & 7.877 \\
1.15 & 583 & 0.041416 & 0.05712 & $2.74 \times 10^{-3}$ & 52.9 & 7.06058 & 9.881 \\
1.25 & 483 & 0.045502 & 0.03453 & $3.46 \times 10^{-3}$ & 73.1 & 5.72235 & 11.71 \\
1.35 & 335 & 0.054636 & 0.1111 & $4.51 \times 10^{-3}$ & 22.2 & 12.3898 & 9.740 \\
\hline
\end{tabular}

Table 7.2 shows the number of candidates, the uncertainties used to calculate $\Delta_{\text {uncorr }}$ as well as the number candidates corrected for purity, efficiency and luminosity, $N_{\text {corr }}$ (obtained using Eq. 7.1) and the systematic (correlated) uncertainty. The correlated uncertainty was calculated similarly to the uncorrelated uncertainty. The uncertainties due to the background shape and the jet $\eta$ position resolution were reported in Chapter 6. These uncertainties along with the luminosity uncertainty were then added in quadrature to obtain the total correlated uncertainty:

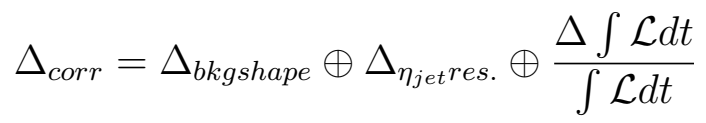

The distribution in Fig. 7.2 shows the angular distribution for 2011 data along with the NLO theoretical prediction. All distributions are normalized with respect to their 
integrals. The error bars on the data points represent the combined correlated and uncorrelated uncertainties. The NLO theoretical predication is shown in histogram format along with its uncertainty due to number of entries and the assigned weighting. The ratio at the bottom of Fig. 7.2 shows that there is little deviation between the experimental angular distribution and its theoretical prediction at NLO with the exception of the last point. It is shown that the theory error bars (due to statistics and assigned weighting) borders the total uncertainties calculated for data, concluding that given enough statistics in the theoretical prediction there could be better agreement between data and theory.

The uncorrelated uncertainty varies between 3.7-12.3\% with the largest uncertainty contributing to the $14^{\text {th }}$ bin. The correlated uncertainty varies between $4.6-11.7 \%$. Beyond $\left|\eta^{*}\right|>1.4$ it is difficult to validate the data-driven technique used to obtain the background template for this study due to the fact that there are not enough events to obtain a well-defined $\sigma_{i \eta i \eta}$ distribution to compare between the data-driven template and QCD MC.

\subsection{Comparison with Other Next-to-Leading Order Calculations}

It was brought to light during the angular distribution analysis of $\mathrm{Z}+$ jet events [61, 62] in which only the exclusive one-jet process is examined that there is a disagreement in theory between the PYTHIA [53], MADGRAPH [63] and SHERPA [64] event generators as shown in Fig. 7.4 for the combined electron and muon channels. For comparison with the $\mathrm{Z}+$ jet analysis, the NLO theoretical prediction used in this analysis (discussed in the previous section) was compared with the predictions given by MADGRAPH and SHERPA at the generator level. The top distribution on Fig. 7.3 shows the $\left|\eta^{*}\right|$ distribution for data overlaid with the NLO prediction used for this analysis (labeled as 'Owens'), the SHERPA event generator and the MADGRAPH event generator. The 
MADGRAPH distribution shows a higher value at low compared to our reference NLO QCD prediction while SHERPA reports a lower value in the same region. At a value of $\left|\eta^{*}\right|=0.5$, the roles are reversed and is shown to underestimate the distribution compared with Owen's NLO prediction while SHERPA overestimates the distribution.

The discrepancy observed in SHERPA is suspected to be the result of the value set on the matching parameter, which associates matrix elements to parton showers, at the time of event generation [65]. Events were generated such that a photon may be associated with one or more (up to four) jets in the event. These jets may or may not originate from the same parton shower, Fig. 7.5 shows a visual representation of the situation. The matching parameter avoids such double parton counting to occur and assigns each jet to the correct parton shower it originated from [66]. This parameter differed between event generators, which leads to comparing different matrix elements between the NLO predictions and thus causing an inconsistency between SHERPA, MADGRAPH and Owens.

\subsection{Conclusions}

The angular distribution, $\left|\eta^{*}\right|$, is defined as the difference in $\eta$ between the leading photon and leading jet in an event. The angular distribution was measured for the Compact Muon Solenoid experiment with $5.56 \mathrm{fb}^{-1}$ of data recorded during the 2011 run year. This is the first measurement of ss kind in over a decade since the early days of the Tevatron. It is a test of NLO QCD and is directly related to the matrix element, $|\mathcal{M}|^{2}$ for any given cross section calculation.

The HLT_Photon30_CaloIdVL trigger recorded $4.905 \mathrm{pb}^{-1}$ of data which was used to measure the angular distribution. Photons in which its $p_{T}>40 \mathrm{GeV}$ and was contained within $|\eta|<1.4$ were selected for this analysis. The accompanying jet in the event must have $p_{T}>30 \mathrm{GeV}$ and be confined within $|\eta|<2.4$. A data-driven 
technique was used to obtain the background template to calculate the purity of the sample and was validated (up to $\left|\eta^{*}\right|=1.4$ ) by comparing the template distribution with QCD MC. The data is in good agreement with NLO theoretical prediction produced by Baer, Ohnemus and Owens, however discrepancies were discovered when this prediction was compared with MADGRAPH and SHERPA NLO event generators when looking at the exclusive case in which only one jet which satisfy the jet selection criteria is allowed to accompany the photon in an event.

The prompt photon angular distribution measurement is complimentary to such analyses involving the prompt photon cross section and the inclusive jet cross section measurements, but with notable advantages. The prompt photon cross section measurement is coupled to the parton distribution functions, as shown in Eq. 1.3, while the angular distribution measurement is decoupled from PDFs, which result in less sensitivity to the uncertainties associated with the measurement. In measuring the inclusive jet cross section, the jet identification process is more demanding with various algorithms that are currently used to form jets (recall the algorithm used in this analysis was the Anti- $\mathrm{k}_{T}$ algorithm, discussed in Section 3.2.2). The differences in how each jet finding algorithm may need to be further studied in order to validate any differences observed in the experimental results and could cause further complication. Not only is the angular distribution measurement of prompt photons a test of NLO QCD, but it is also a simple and direct measurement of the matrix element, $|\mathcal{M}|^{2}$, the most vital component in any cross section calculation. 


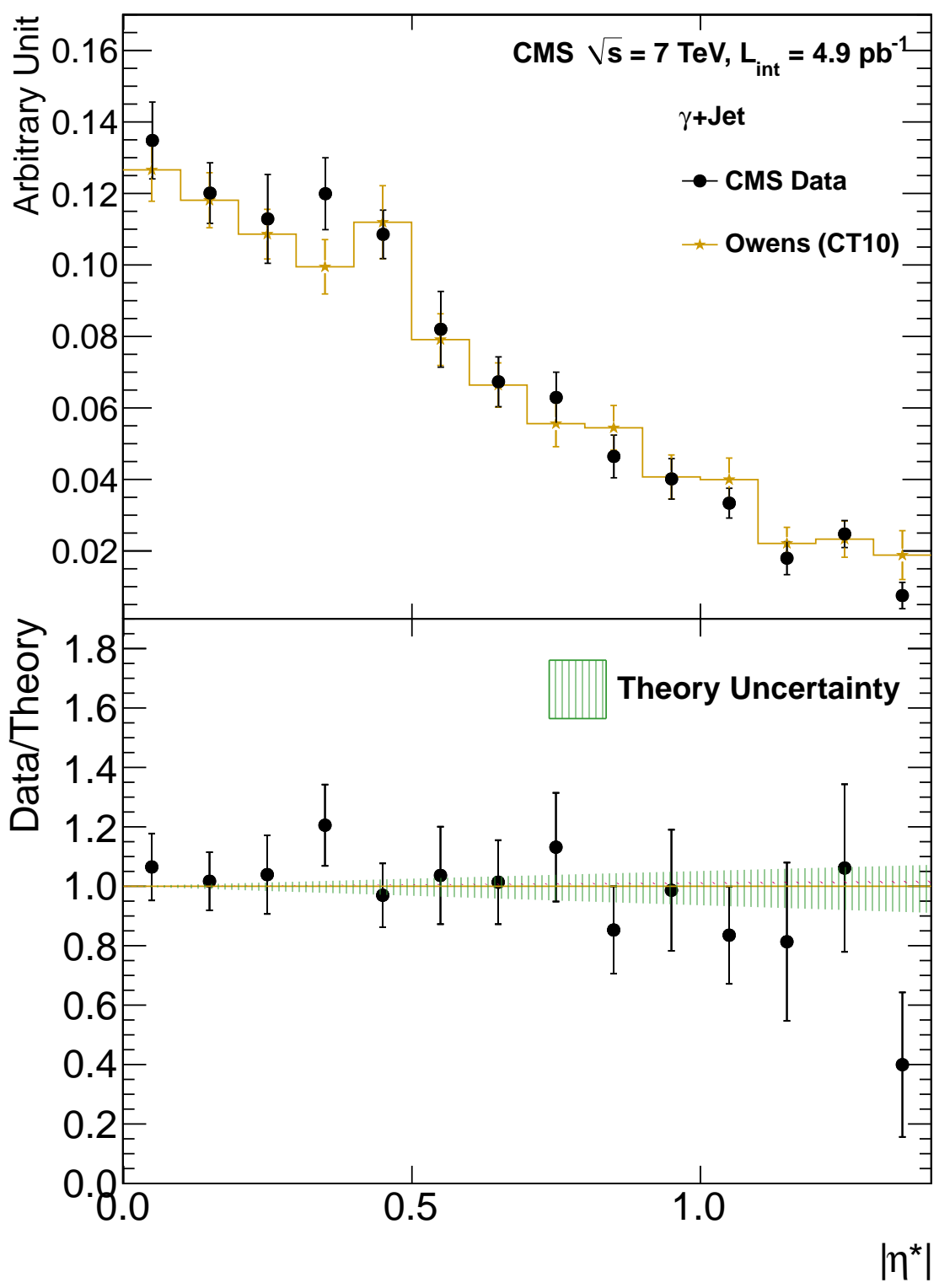

Figure 7.2: The angular distribution, $\left|\eta^{*}\right|$, for 2011 data is represented by the black circles where the error bars represent the combined correlated and uncorrelated uncertainties. The histogram represents the NLO QCD prediction along with its weighted statistical uncertainty. The distribution shown below is the data/theory ratio, showing that data agrees with NLO predictions, with an exception at the last bin where we were statistics limited in the theoretical predication. The striped distribution in the ratio plot is the theoretical uncertainty values shown in Table 7.1 . 


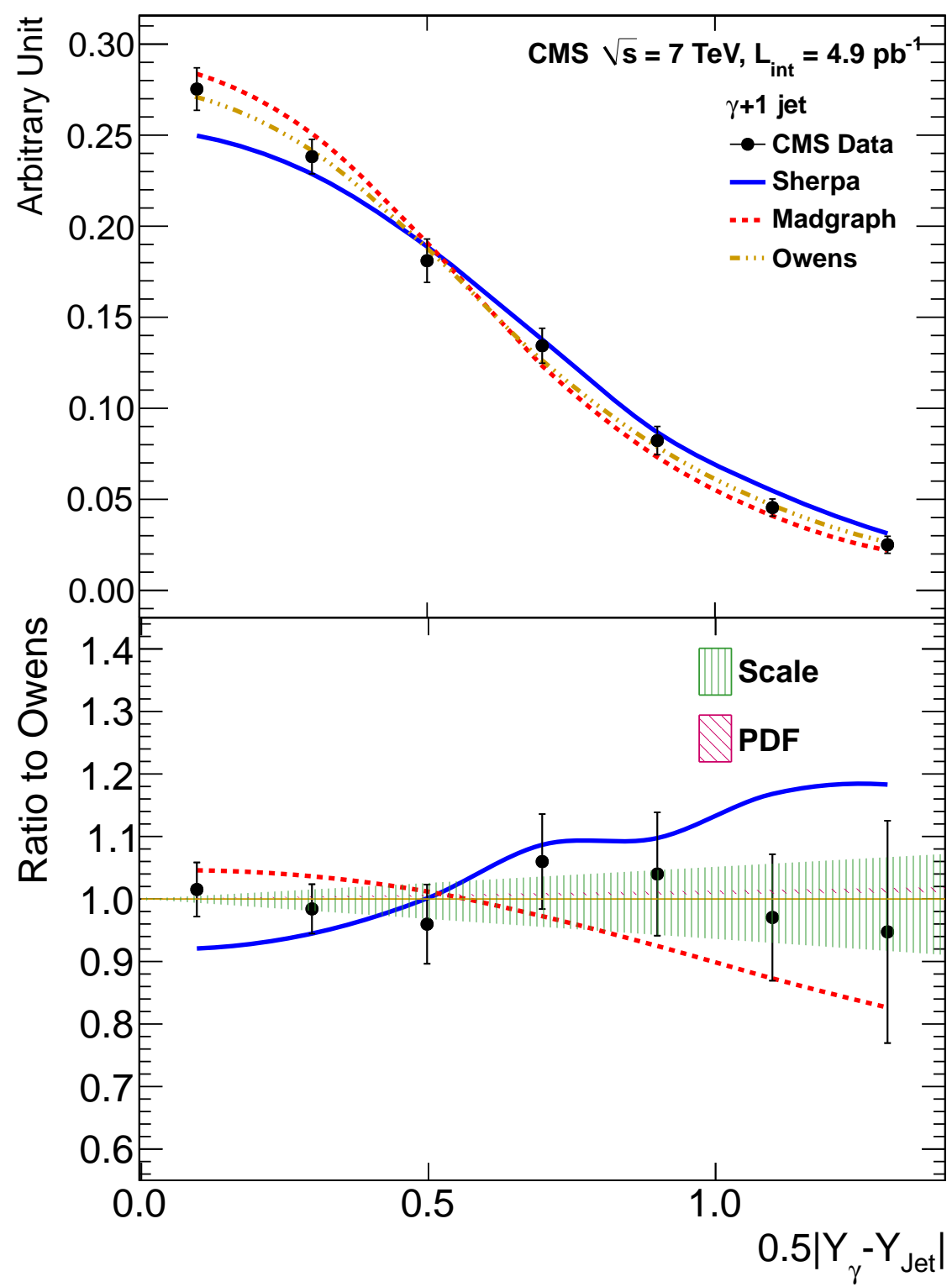

Figure 7.3: The $\left|\eta^{*}\right|$ distribution for data (closed circles) overlaid with three NLO predictions for $\left|\eta^{*}\right|$; Owens (solid-dotted line), SHERPA (solid line) and MADGRAPH (dotted line) for the exclusive case in which only one jet accompanies a photon in an event. 


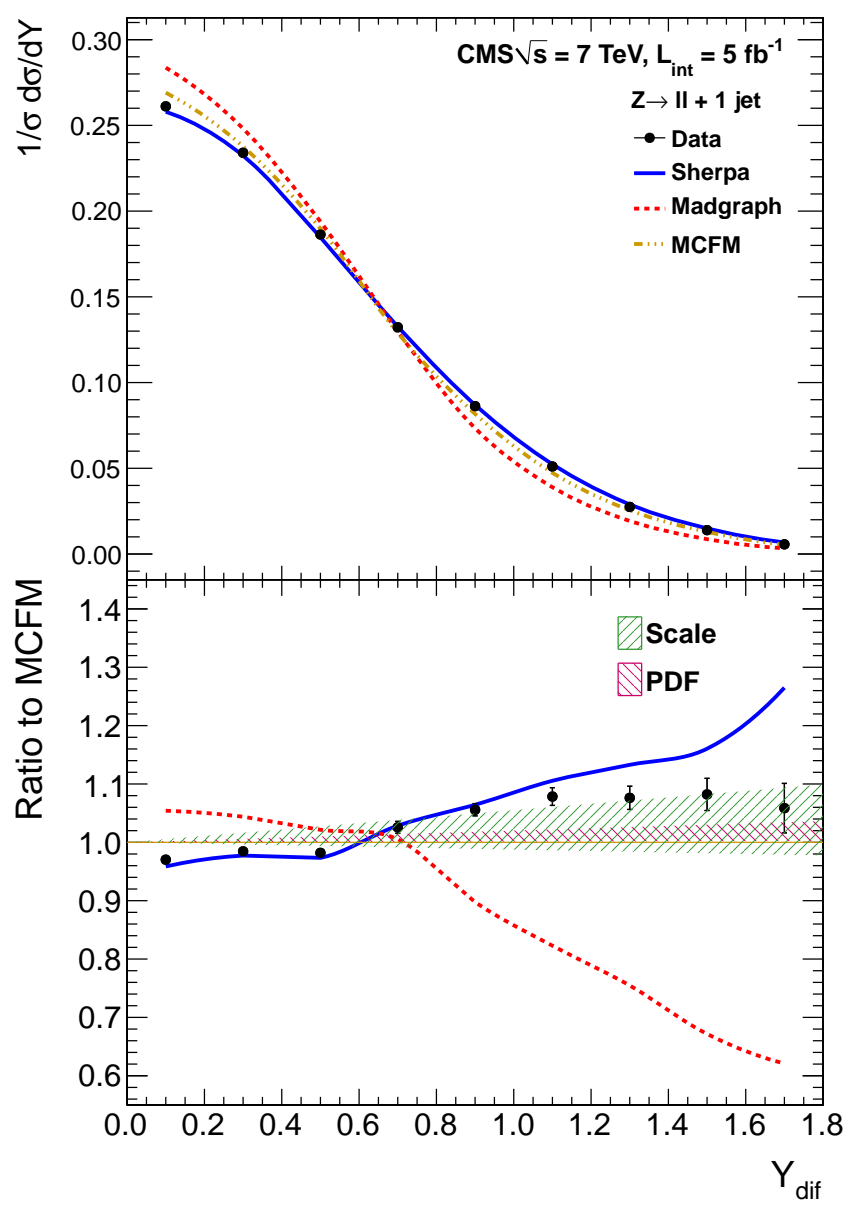

Figure 7.4: Angular distribution in $\mathrm{Z}+$ jet events for the lepton channel $(\rightarrow e e$ and $\rightarrow \mu \mu)$ which shows a discrepancy between the MADGRAPH and SHERPA NLO theoretical predictions and the reference NLO theory prediction (MCFM).
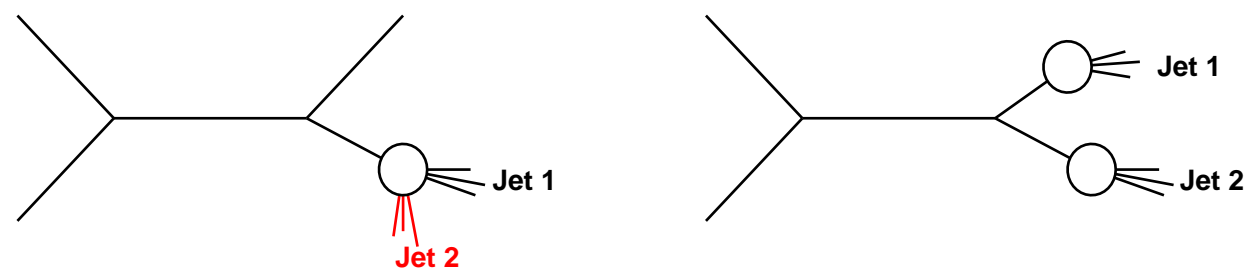

Figure 7.5: Representation of one parton shower forming two jets on the left side. The right side depicts each parton shower forming an individual jet. The matching parameter avoids double counting of parton showers in cases like the left side example where more than one jet may be formed by the same parton shower. 


\section{REFERENCES}

[1] James William Rohlf. Modern Physics from $\alpha$ to $Z^{0}$. John Wiley and Sons, 1994.

[2] K. Nakamura et al. (Particle Data Group) J. Phys. G, 37(075021), 2010.

[3] Dan Green. High $p_{T}$ Physics at Hadron Colliders. Cambridge University Press, 2005.

[4] CMS Collaboration. CMS Physics Technical Design Report Volume I: Detector Performance and Software, volume CERN-LHCC-2006-001. CERN, Geneva, 2006.

[5] Robert Hofstadter. Electron Scattering and Nuclear Structure. Rev. Mod. Phys., 28:214-254, Jul 1956. Available from: 10.1103/RevModPhys.28.214.

[6] Siegfried Bethke. The 2009 World Average of $\alpha_{s}$. Eur. Phys. J. C, 64:689, 2009. Available from: doi:10.1140/epjc/s10052-009-1173-1.

[7] Paul Rubinov. Measurement of the Direct Photon Angular Distribution at DØ. PhD thesis, State University of New York at Stoney Brook, 1995.

[8] F. Abe et al. (CDF Collaboration). Center-of-Mass Angular Distribution of Prompt Photons Produced in $p \bar{p}$ Collisions at $\sqrt{s}=1.8$ Tev. Phys. Rev. Lett., 71:679-683, Aug 1993. Available from: 10.1103/PhysRevLett.71.679.

[9] J.F. Owens. Large-Momentum-Transfer Production of Direct Photons, Jets, and Particles. Rev. Mod. Phys.; Reviews of Modern Physics, 59(2):465-503, 1987. Available from: 10.1103/RevModPhys.59.465.

[10] H. Baer, J. Ohnemus, and J. F. Owens. A Next-to-Leading-Logarithm Calculation of Jet Photoproduction. Phys. Rev. D, 42:61-71, Jul 1990. Available from: 10.1103/PhysRevD.42.61.

[11] European Organization for Nuclear Research. The CERN Accelerator Complex, 2006. Available from: http://homepage.mac.com/fishbonelpc/CERN/public. web.cern.ch/Public/Content/Chapters/AskAnExpert/LHC-en.html\#qa3.

[12] CMS Collaboration. CMS ECAL Technical Design Report, volume CERNLHCC-97-33. CERN, Geneva, 1997. Available from: http://cmsdoc.cern.ch/ $\mathrm{cms} / \mathrm{TDR} / \mathrm{ECAL} /$ ecal.html.

[13] Matteo Cacciari, Gavin P. Salam, and Gregory Soyez. The Anti- $\mathrm{k}_{T}$ Jet Clustering Algorithm. Journal of High Energy Physics, 2008(04):063, 2008. Available from: http://stacks.iop.org/1126-6708/2008/i=04/a=063.

[14] CMS Collaboration. Jet Performance in pp Collisions at 7 TeV. CMS-PAS-JME10-003, 2010. Available from: http://cdsweb.cern.ch/record/1279362.

[15] CMS Luminosity Official Results, 2011. Available from: https://twiki.cern.ch/ twiki/bin/view/CMSPublic/LumiPublicResults2011. 
[16] CMS Collaboration. Particle-Flow Event Reconstruction in CMS and Performance for Jets, Taus, and MET. CMS-PAS-PFT-09-001, Apr 2009. Available from: http://cdsweb.cern.ch/record/1194487.

[17] H. Geiger and E. Marsden. On a Diffuse Reflection of the $\alpha$-Particle. Available from: 10.1098/rspa.1909.0054.

[18] Robert Hofstadter-Biography, 2012. Available from: http://www.nobelprize. org/nobel_prizes/physics/laureates/1961/hofstadter-bio.html.

[19] Beat Hahn, D. G. Ravenhall, and Robert Hofstadter. High-Energy Electron Scattering and the Charge Distributions of Selected Nuclei. Phys. Rev., 101:11311142, Feb 1956. Available from: http://link.aps.org/doi/10.1103/PhysRev.101. 1131 .

[20] Hans F. Ehrenberg, Robert Hofstadter, Ulrich Meyer-Berkhout, D. G. Ravenhall, and Stanley E. Sobottka. High-Energy Electron Scattering and the Charge Distribution of Carbon-12 and Oxygen-16. Phys. Rev., 113:666-674, Jan 1959. Available from: 10.1103/PhysRev.113.666.

[21] W.N. Cottingham and D.A. Greenwood. An Introduction to the Standard Model of Particle Physics. Cambridge University Press, 1998.

[22] Francis Halzen and Alan D. Martin. Quarks and Leptons: An Introductory Course in Modern Particle Physics. John Wiley and Sons, 1984.

[23] David Griffiths. Introduction to Particle Physics. John Wiley and Sons, 1987.

[24] Raymond A. Serway, Clement J. Moses, and Curt A. Moyer. Modern Physics. Thompson Learning, 1997.

[25] Steven Weinberg. A Model of Leptons. Phys. Rev. Lett., 19(21):1264-1266, 1967. Available from: 10.1103/PhysRevLett.19.1264.

[26] Donald H. Perkins. Introduction to High Energy Physics. Addison-Wesley Publishing Company, Inc., 1987.

[27] Observation of A New Particle With A Mass of $125 \mathrm{GeV}$. Available from: http: //cms.web.cern.ch/news/observation-new-particle-mass-125-gev.

[28] CMS Collaboration. Combination of Higgs Searches. CMS-PAS-HIG-11-022, 2011. Available from: http://cdsweb.cern.ch/record/1376643.

[29] R.K. Ellis, W.J. Stirling, and B.R. Webber. QCD and Collider Physics. Cambridge University Press, 2003.

[30] Rick Field. Early LHC Underlying Event Data - Findings and Surprises. 2010. Available from: http://arxiv.org/abs/1010.3558v1. 
[31] Carter Randolph Hall. Measurement of the Isolated Direct Photon Cross Section with Conversions in $p \bar{p}$ Collisions at $\sqrt{s}=1.8$ TeV. PhD thesis, Harvard University, 2002.

[32] CMS Collaboration. Measurement of the Differential Cross Section for Isolated Prompt Photon Production in pp Collisions at 7 TeV. 08 2011. Available from: http://arxiv.org/abs/1108.2044v1.

[33] T. Aaltonen et al. (CDF Collaboration). Measurement of the Inclusive Isolated Prompt Photon Cross Section in $\mathrm{p}$ anti-p Collisions at $s^{1 / 2}=1.96-\mathrm{TeV}$ using the CDF Detector. Phys.Rev., D80:111106, 2009. Available from: 10.1103/ PhysRevD.80.111106.

[34] F. Abe et al. (CDF Collaboration). Precision Measurement of the Prompt Photon Cross Section in $p \bar{p}$ Collisions at $\sqrt{s}=1.8$ tev. Phys. Rev. Lett., 73:26622666, Nov 1994. Available from: http://link.aps.org/doi/10.1103/PhysRevLett. 73.2662 .

[35] F. Abe et al. (CDF Collaboration). A Prompt Photon Cross-Section Measurement in $\bar{p} p$ Collisions at $\sqrt{s}=1.8 \mathrm{TeV}$. Phys.Rev., D48:2998-3025, 1993. Available from: $10.1103 /$ PhysRevD.48.2998.

[36] V. M. Abazov et al. (DØ Collaboration). The Ratio of the Isolated Photon Cross Sections at $\sqrt{s}=630 \mathrm{GeV}$ and $1800 \mathrm{GeV}$. Phys.Rev.Lett., 87:251805, 2001. Available from: 10.1103/PhysRevLett.87.251805.

[37] S. Abachi et al. (DØ Collaboration). Isolated Photon Cross-Section in the Central and Forward Rapidity Regions in $p \bar{p}$ Collisions at $\sqrt{s}=1.8 \mathrm{TeV}$. Phys.Rev.Lett., 77:5011-5015, 1996. Available from: 10.1103/PhysRevLett.77.5011.

[38] S. van der Meer. Calibration of the Effective Beam Height in the ISR. Technical Report CERN-ISR-PO-68-31, CERN, Geneva, 1968. Available from: http:// cdsweb.cern.ch/record/296752.

[39] D. Belohrad et al. The LHC Fast BCT System: A Comparison of Design Parameters with Initial Performance. Technical Report CERN-BE-2010-010, CERN, Geneva, May 2010.

[40] Oliver Brüning et al. LHC Design Report. CERN, Geneva, 2004. Available from: http://cdsweb.cern.ch/record/782076.

[41] CMS Collaboration. Performance and Operation of the CMS Electromagnetic Calorimeter. Journal of Instrumentation, 5(03):T03010, 2010. Available from: http://stacks.iop.org/1748-0221/5/i=03/a=T03010.

[42] Daniele Passeri et al. Characterization of CMOS Active Pixel Sensors for Particle Detection: Beam Test of the Four-Sensors RAPS03 Stacked System. Available from: 10.1016/j.nima.2009.10.067. 
[43] Alan M. Litke and Andreas S. Schwartz. The Silicon Microstrip Detector. Scientific American, 275(5), 1995.

[44] CMS Collaboration. The CMS Tracker System Project : Technical Design Report. CERN-LHCC-98-006, 1998. Available from: http://cdsweb.cern.ch/ record $/ 368412 ? \ln =\mathrm{en}$.

[45] Tejinder S. Virdee. Calorimetry. In Techniques and Concepts of High Energy Physics X, pages 335-386, Boston, Massachusetts, 1999. Kluwer Academic Publishers.

[46] CMS Collaboration. CMS Electromagnetic Trigger Commissioning and First Operation Experiences. J. Phys: Conf. Ser, 160(012062), 2009.

[47] CMS Collaboration. CMS Computing: Technical Design Report. CERN-LHCC2005-023, 2005. Available from: http://cdsweb.cern.ch/record/838359/files/ lhcc-2005-023.pdf.

[48] Ruth Pordes et al. The Open Science Grid. Journal of Physics: Conference Series, 78(1):012057, 2007. Available from: http://stacks.iop.org/1742-6596/ $78 / \mathrm{i}=1 / \mathrm{a}=012057$.

[49] D. Spiga et al. CRAB: The CMS Distributed Analysis Tool Development and Design. Available from: http://dx.doi.org/10.1016/j.nuclphysbps.2007.11.124.

[50] S. Catani et al. Longitudinally-Invariant $\mathrm{k}_{\perp}$-Clustering Algorithms for HadronHadron Collisions. Nucl. Phys. B, 406(CERN-TH-6775-93, LU-TP-93-2):187224, 1993.

[51] Yuri L. Dokshitzer et al. Better Jet Clustering Algorithm. Available from: http://arxiv.org/abs/hep-ph/9707323.

[52] S. Agostinelli et al. Geant4 A Simulation Toolkit. Nuclear Instruments and Methods in Physics Research Section A: Accelerators, Spectrometers, Detectors and Associated Equipment, 506(3):250 - 303, 2003. Available from: 10.1016/ S0168-9002(03)01368-8.

[53] Torbjörn Sjöstrand, Stephan Mrenna, and Peter Skands. PYTHIA 6.4 Physics and Manual. Journal of High Energy Physics. Available from: 10.1088/ 1126-6708/2006/05/026.

[54] Summer 2011 CMS Monte Carlo Production, 2011. Available from: https:// twiki.cern.ch/twiki/bin/view/CMS/ProductionSummer2011.

[55] CMS Collaboration. Study of $\mathrm{W} / \gamma$ and $\mathrm{Z} / \gamma$ production at CMS with $\sqrt{s}=7$ TeV. CMS Internal Note AN-2011/251, 2011.

[56] Michael B. Anderson. High- $p_{T}$ Photon Production in Association with Jets at $\sqrt{s}=7$ TeV using the Compact Muon Solenoid at the Large Hadron Collider. PhD thesis, University of Wisconsin, Madison, 2011. 
[57] CMS Collaboration. Performance of the CMS Level-1 Trigger during Commissioning with Cosmic Ray Muons. 11. Available from: http://arxiv.org/abs/0911. 5422v2.

[58] Philip R. Bevington and Keith Robinson. Data Reduction and Error Analysis For the Physical Sciences. McGraw-Hill, New York, 1992.

[59] H. Baer, J. Ohnemus, and J. F. Owens. Next-to-leading-logarithm calculation of direct photon production. Phys. Rev. D, 42:61-71, Jul 1990.

[60] CMS Collaboration. Measurement of CMS Luminosity. CMS-PAS-EWK-10-004, 2011. Available from: http://cdsweb.cern.ch/record/1279145.

[61] CMS Collaboration. Angular Distribution of Z Bosons in Z+Jet Events Using 2011 Data. CMS Internal Note AN-2012/037, 2012.

[62] CMS Collaboration. Measurement of $\mathrm{Z} / \gamma^{*}+$ jet $+\mathrm{X}$ Angular Distributions in pp Collisions at $\sqrt{s}=7 \mathrm{TeV}$ in the Electron Channel. CMS Internal Note AN2012/135, 2012 .

[63] Johan Alwall et al. Madgraph 5: Going Beyond. 2011. Available from: http: //arxiv.org/abs/1106.0522.

[64] T. Gleisberg et al. Event Generation with SHERPA 1.1. Journal of High Energy Physics, 2009(02):007, 2009. Available from: http://stacks.iop.org/1126-6708/ $2009 / \mathrm{i}=02 / \mathrm{a}=007$.

[65] S. Catani, F. Krauss, R. Kuhn, and B.R. Webber. QCD Martix Elements and Parton Showers. 2001. Available from: http://iopscience.iop.org/1126-6708/ 2001/11/063.

[66] Stefan Hoeche, Frank Krauss, Steffen Schumann, and Frank Siegert. QCD Matrix Elements and Truncated Showers. JHEP, 05:053, 2009. Available from: 10.1088/ 1126-6708/2009/05/053.

[67] Andreas Hoecker and Vakhtang Kartvelishvili. SVD Approach to Data Unfolding. Nucl. Instrum. Meth. A, 372:469-481. Available from: http://www. sciencedirect.com/science/article/pii/0168900295014780.

[68] Tim Adye. Unfolding Algorithms and Tests Using RooUnfold. 2011. Available from: http://arxiv.org/abs/1105.1160.

[69] RooUnfold: ROOT Unfolding Framework. Available from: http://hepunx.rl.ac. uk/ adye/software/unfold/RooUnfold.html.

[70] G. D'Agostini. A Multidimensional Unfolding Method Based on Bayes' Theorem. Nucl. Instrum. Meth., A362:487-498, 1995. Available from: 10.1016/ 0168-9002(95)00274-X. 


\section{APPENDIX A}

\section{LEADING PHOTON AND LEADING JET KINEMATICS}

The following distributions represent the kinematic distributions of the leading photon and leading jet for each bin in $\left|\eta^{*}\right|$ for the 2011 data collected using the HLT_Photon30_CaloIdVL trigger (Due to sizing they begin on the following page).
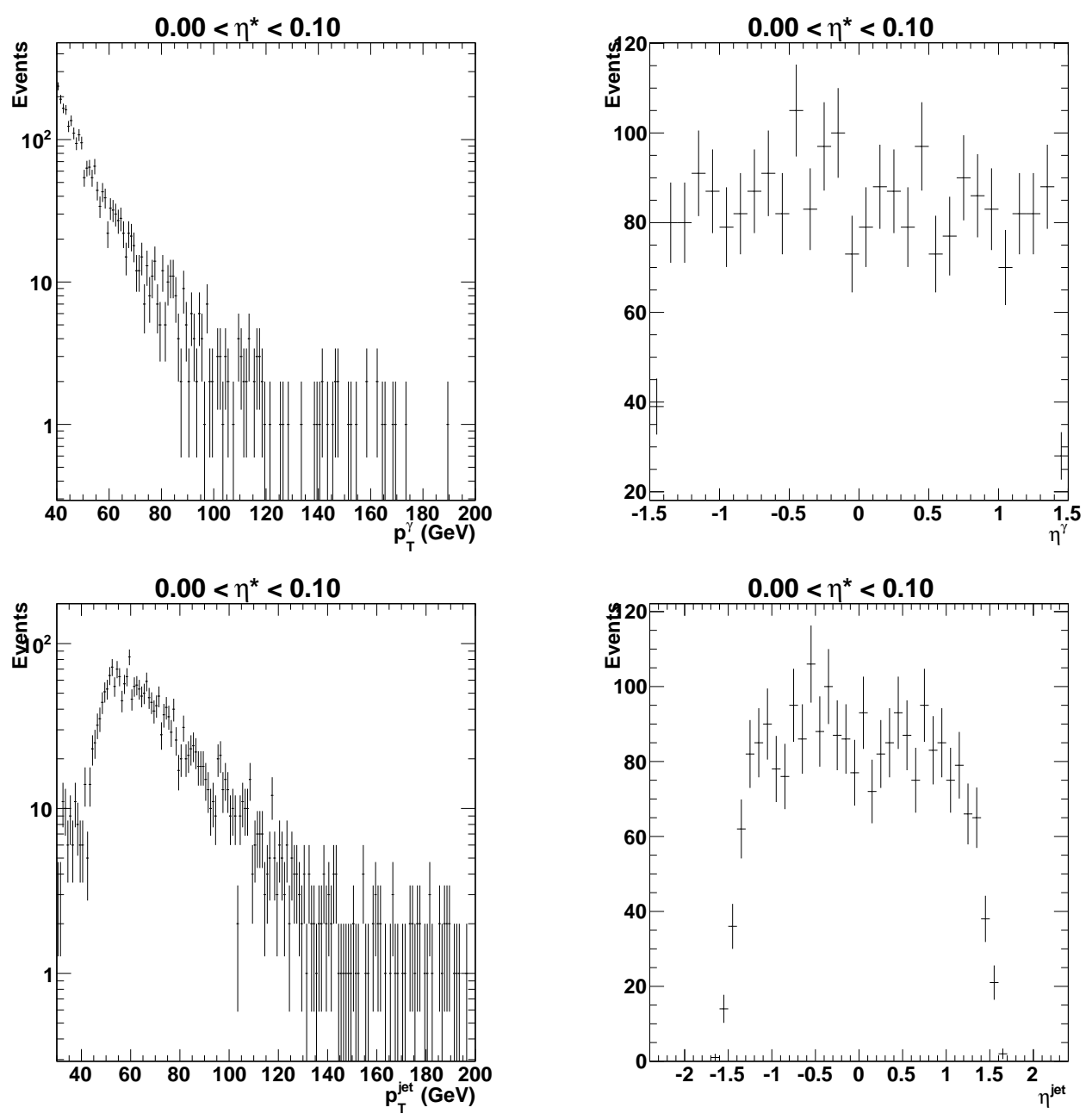

Figure A.1: The leading photon's $p_{T}$ and $\eta$ distributions (top) and the leading jet's $p_{T}$ and $\eta$ distributions on bottom for candidates with $0.0<\left|\eta^{*}\right|<0.1$. 

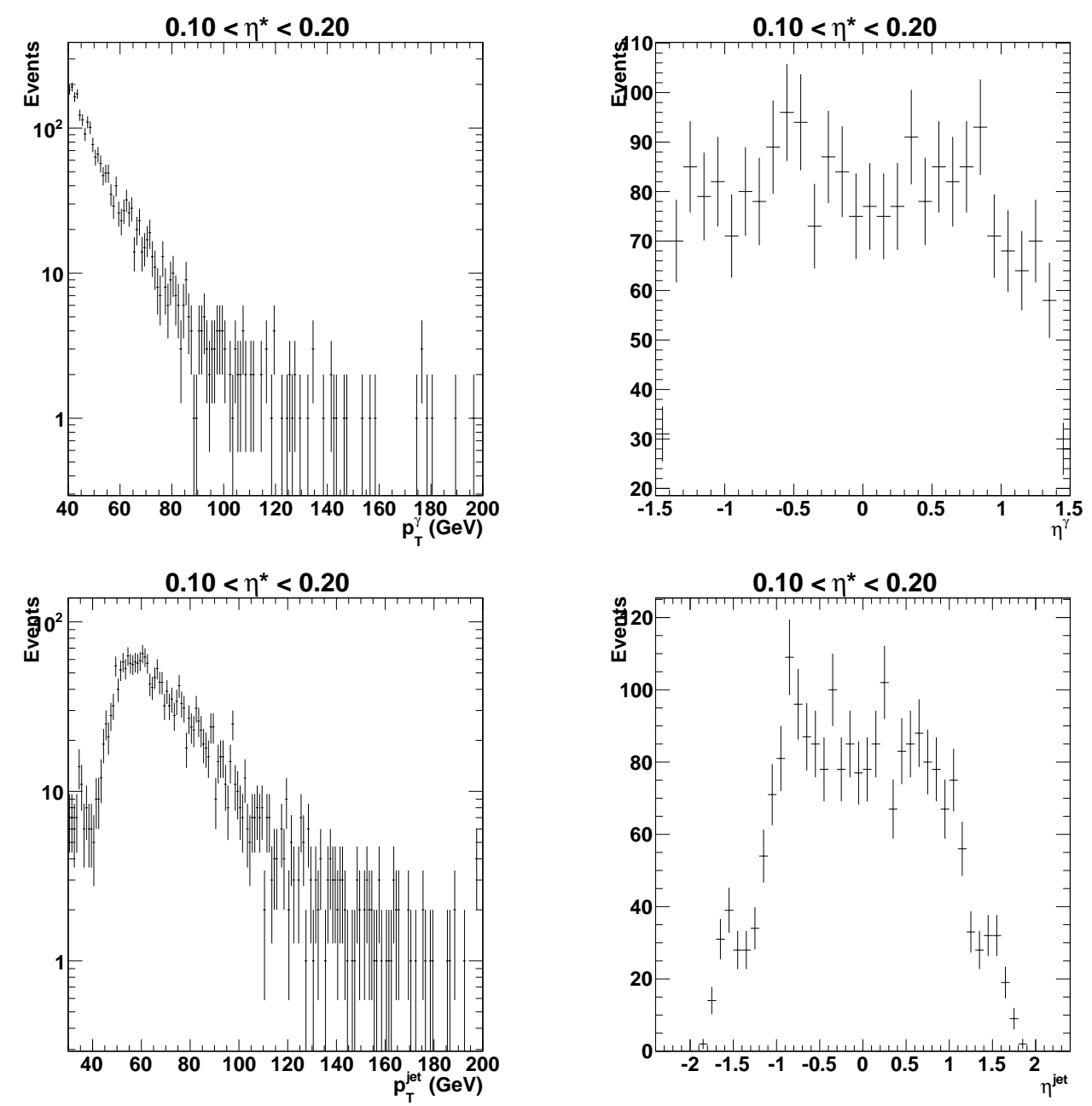

Figure A.2: The leading photon's $p_{T}$ and $\eta$ distributions (top) and the leading jet's $p_{T}$ and $\eta$ distributions on bottom for candidates with $0.1<\left|\eta^{*}\right|<0.2$. 

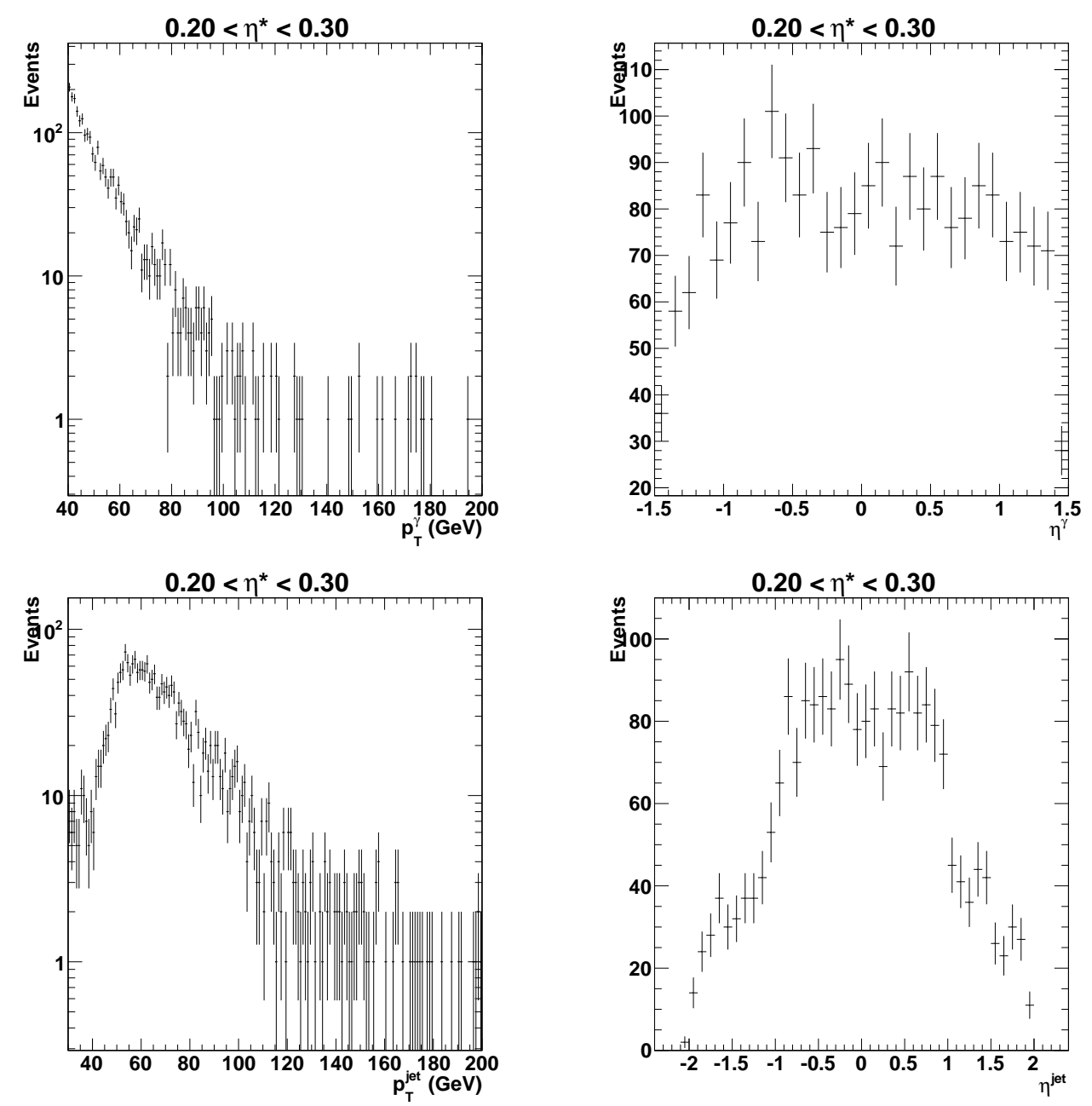

Figure A.3: The leading photon's $p_{T}$ and $\eta$ distributions (top) and the leading jet's $p_{T}$ and $\eta$ distributions on bottom for candidates with $0.2<\left|\eta^{*}\right|<0.3$. 

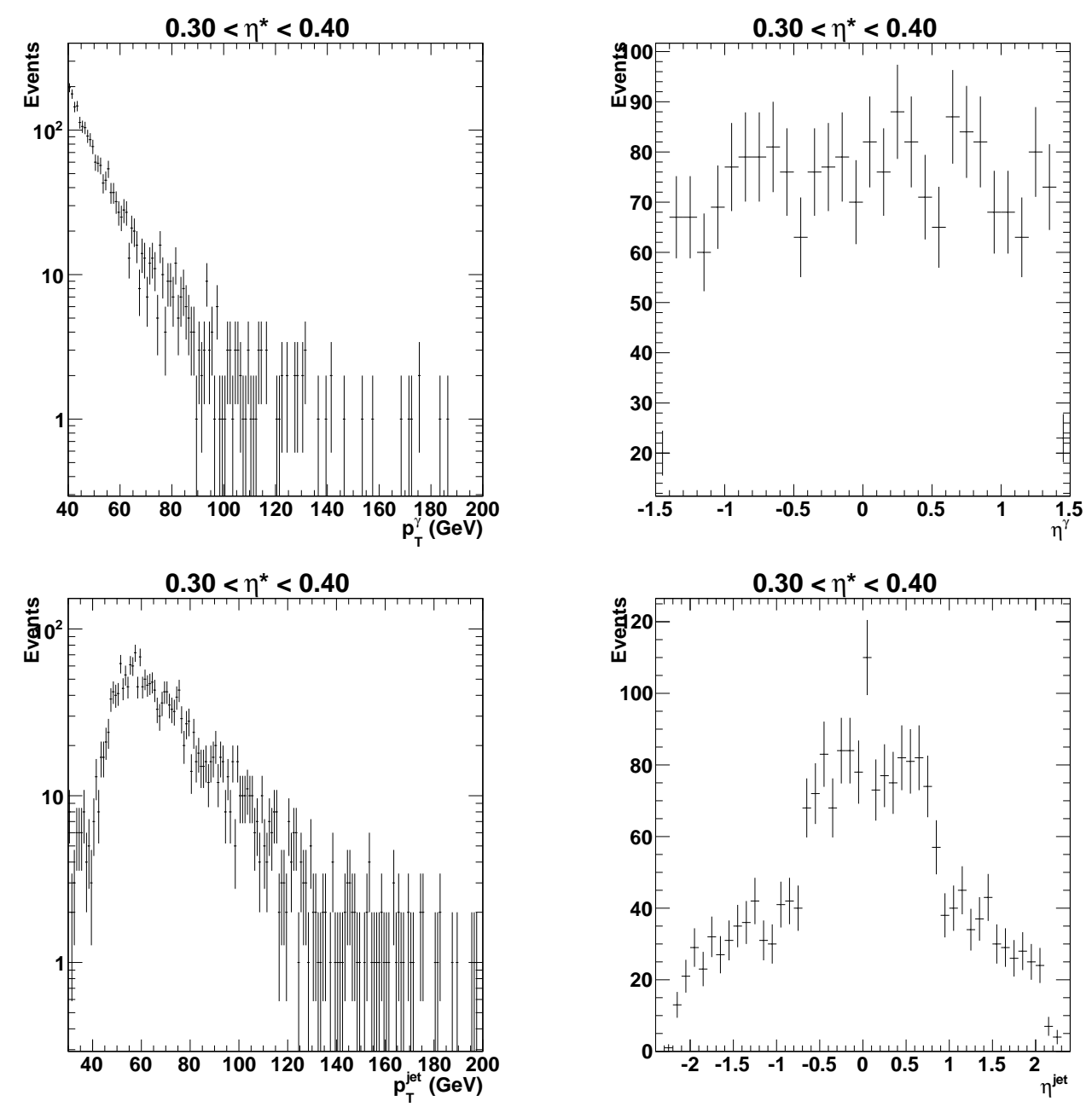

Figure A.4: The leading photon's $p_{T}$ and $\eta$ distributions (top) and the leading jet's $p_{T}$ and $\eta$ distributions on bottom for candidates with $0.3<\left|\eta^{*}\right|<0.4$. 

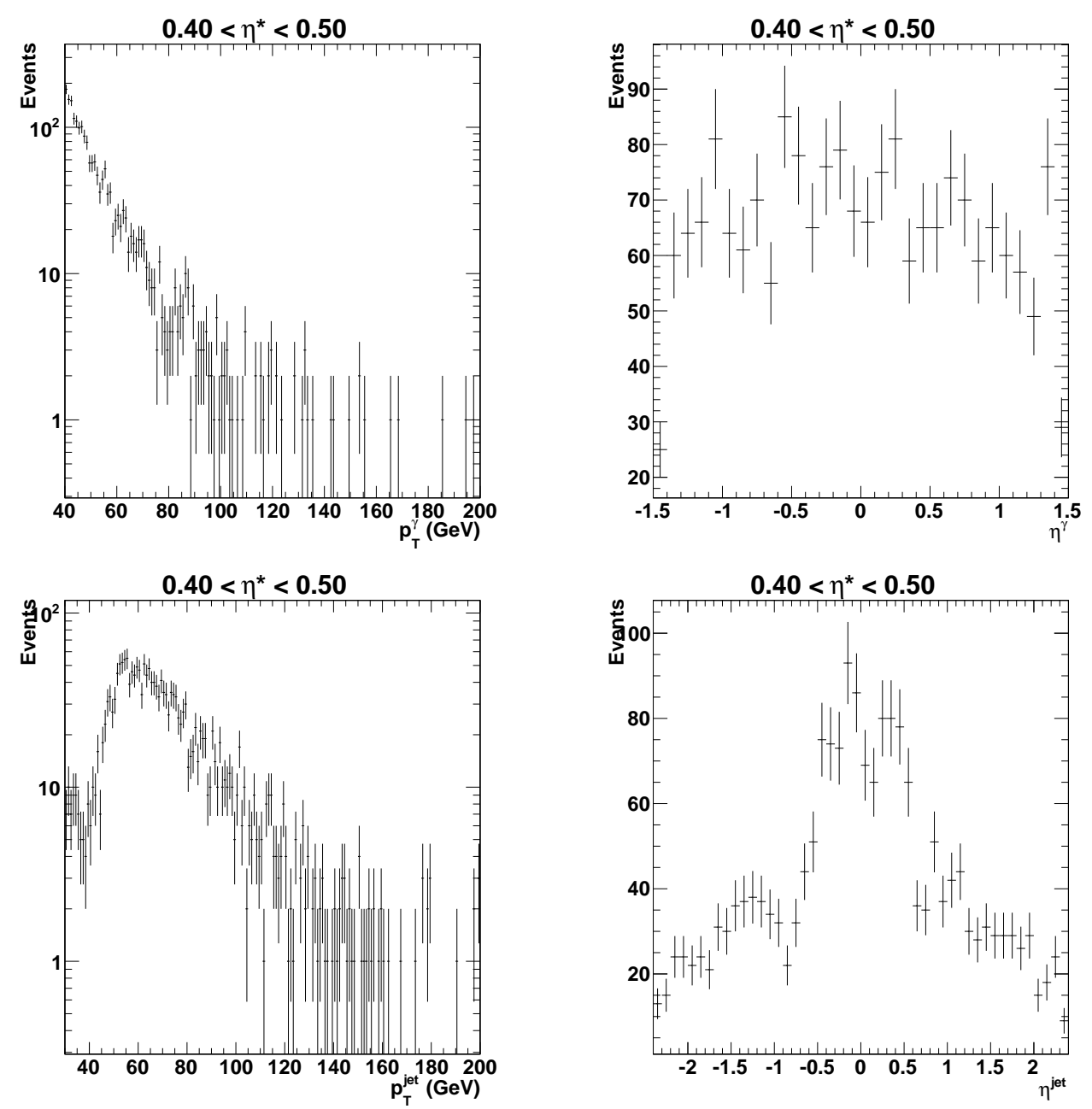

Figure A.5: The leading photon's $p_{T}$ and $\eta$ distributions (top) and the leading jet's $p_{T}$ and $\eta$ distributions on bottom for candidates with $0.4<\left|\eta^{*}\right|<0.5$. 

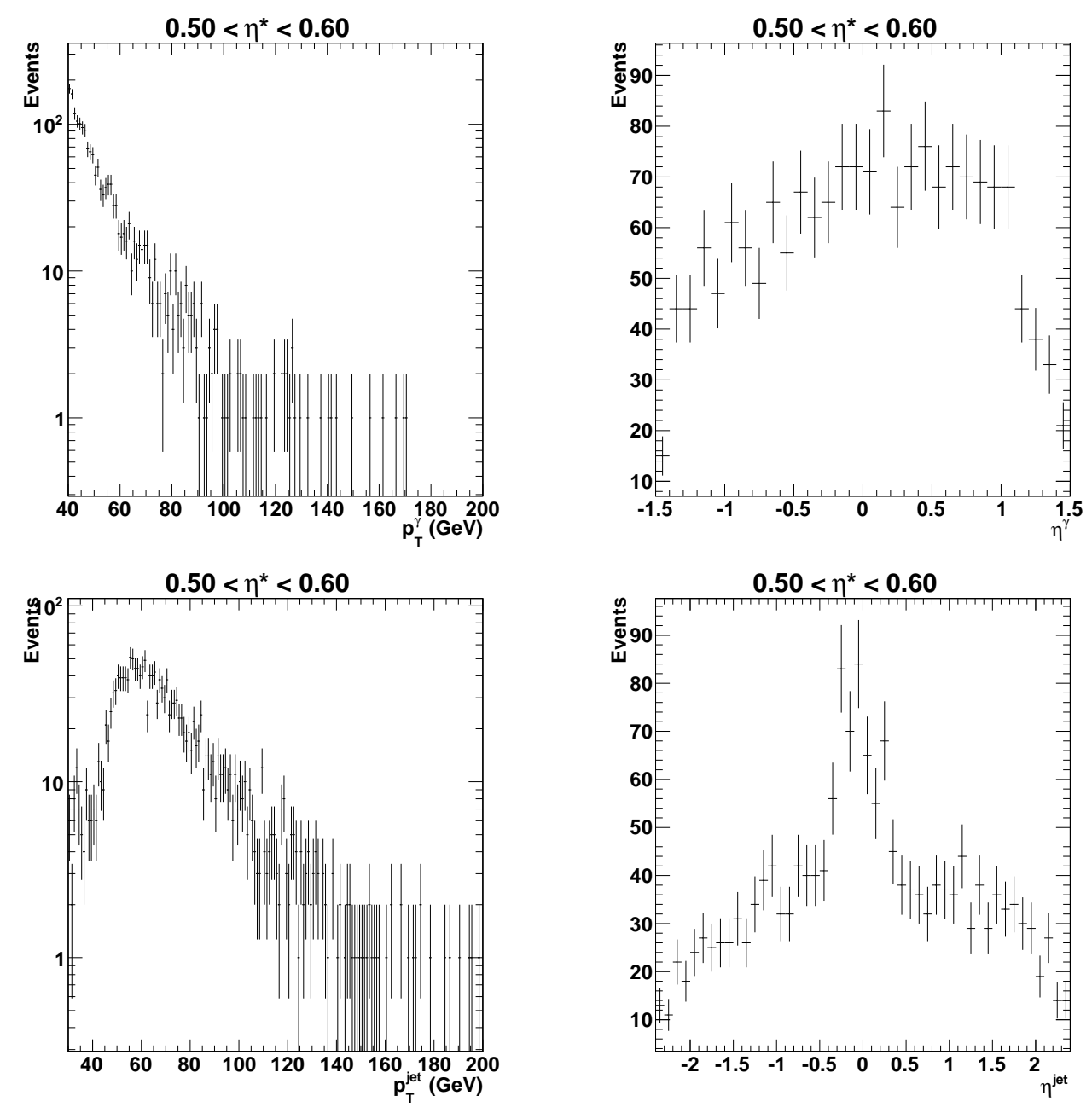

Figure A.6: The leading photon's $p_{T}$ and $\eta$ distributions (top) and the leading jet's $p_{T}$ and $\eta$ distributions on bottom for candidates with $0.5<\left|\eta^{*}\right|<0.6$. 

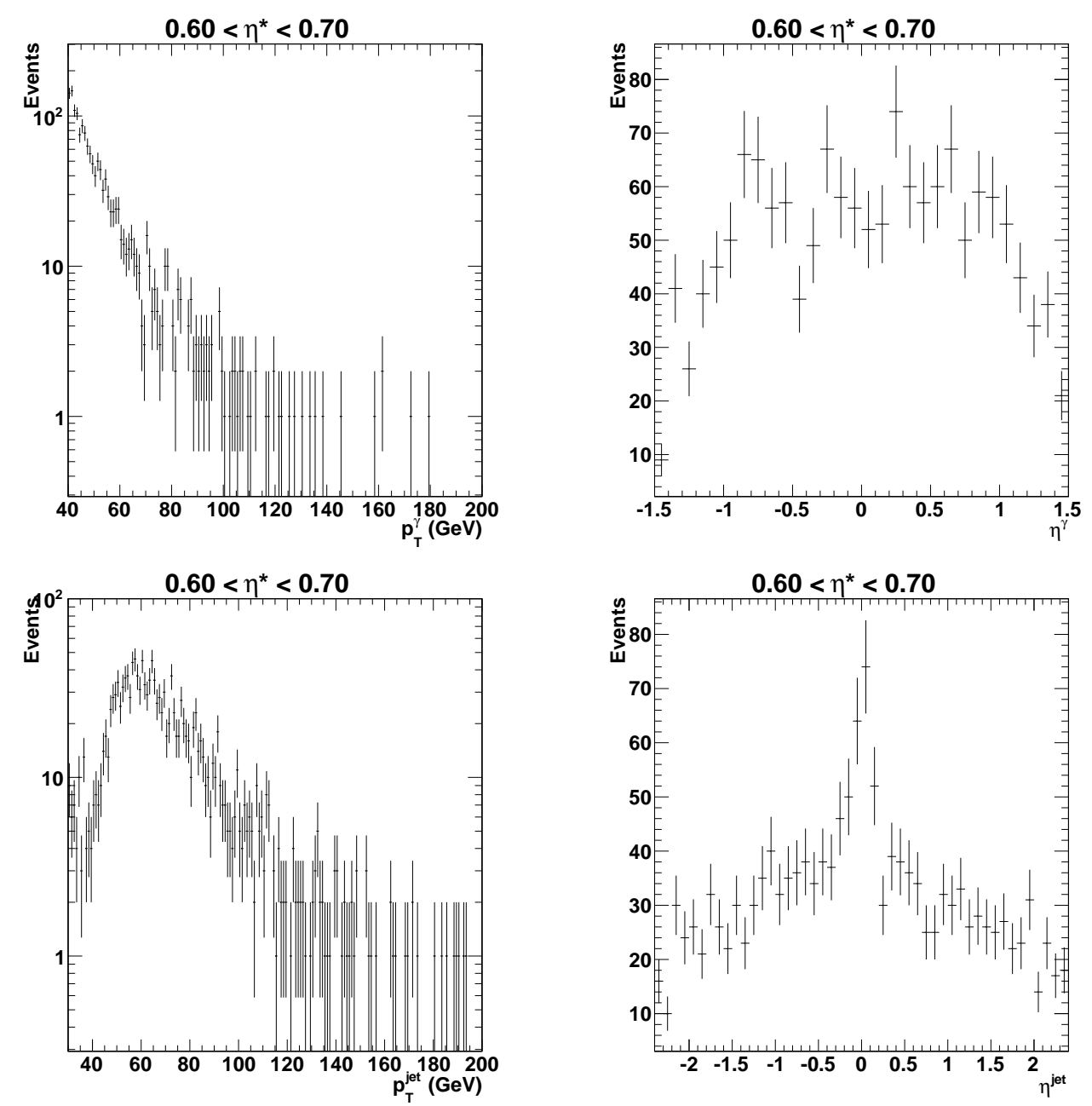

Figure A.7: The leading photon's $p_{T}$ and $\eta$ distributions (top) and the leading jet's $p_{T}$ and $\eta$ distributions on bottom for candidates with $0.6<\left|\eta^{*}\right|<0.7$. 

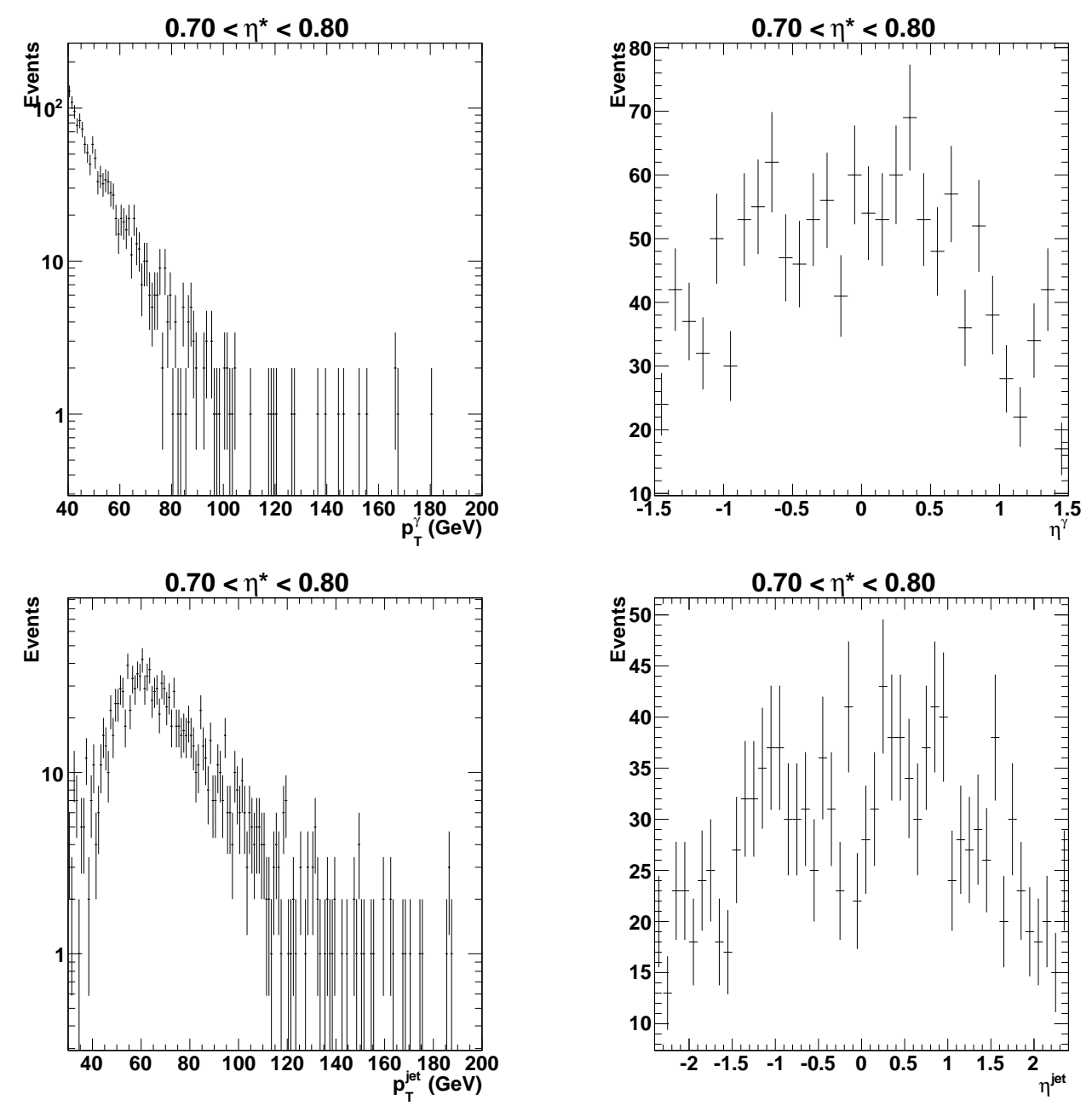

Figure A.8: The leading photon's $p_{T}$ and $\eta$ distributions (top) and the leading jet's $p_{T}$ and $\eta$ distributions on bottom for candidates with $0.7<\left|\eta^{*}\right|<0.8$. 

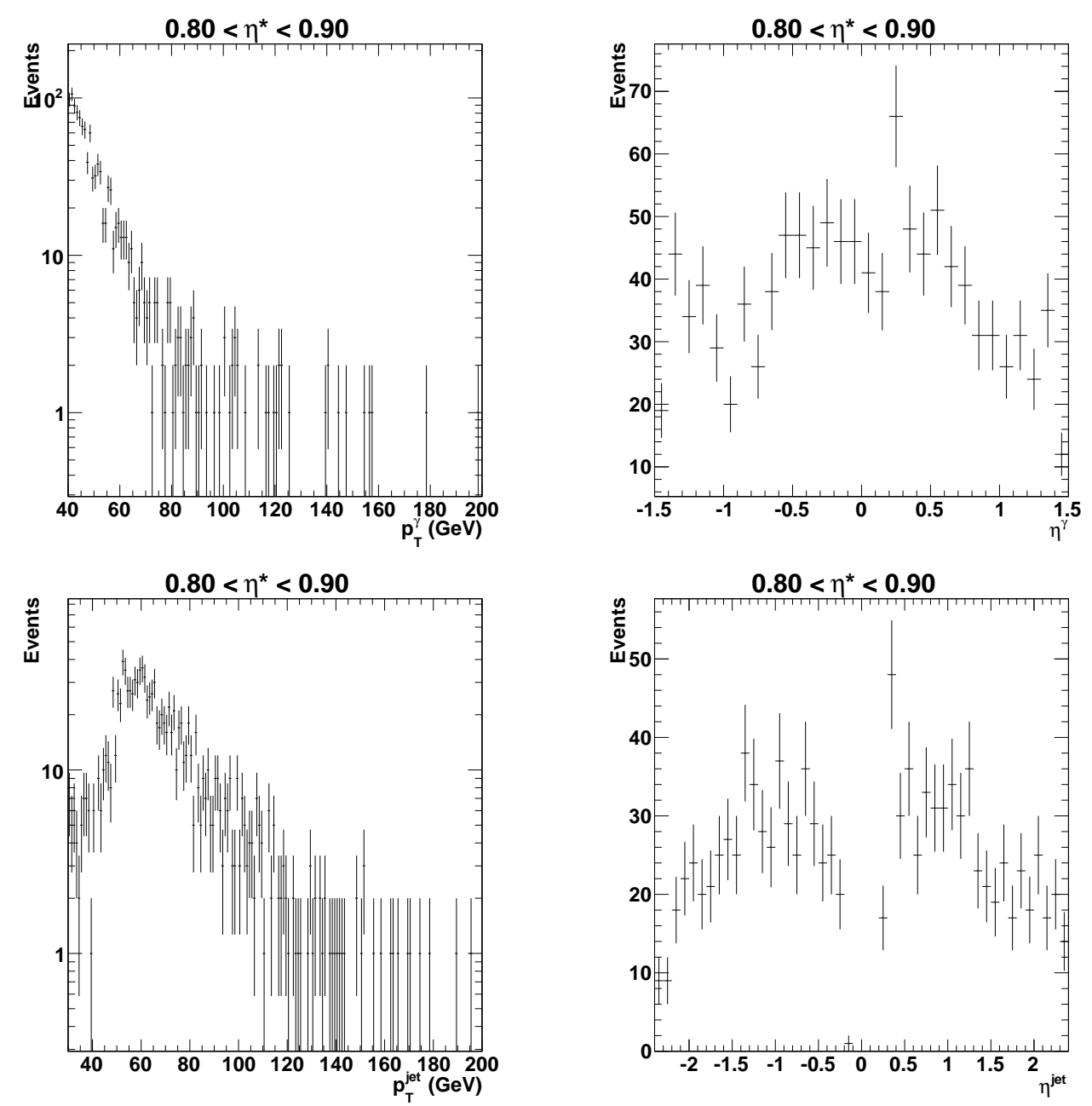

Figure A.9: The leading photon's $p_{T}$ and $\eta$ distributions (top) and the leading jet's $p_{T}$ and $\eta$ distributions on bottom for candidates with $0.8<\left|\eta^{*}\right|<0.9$. 

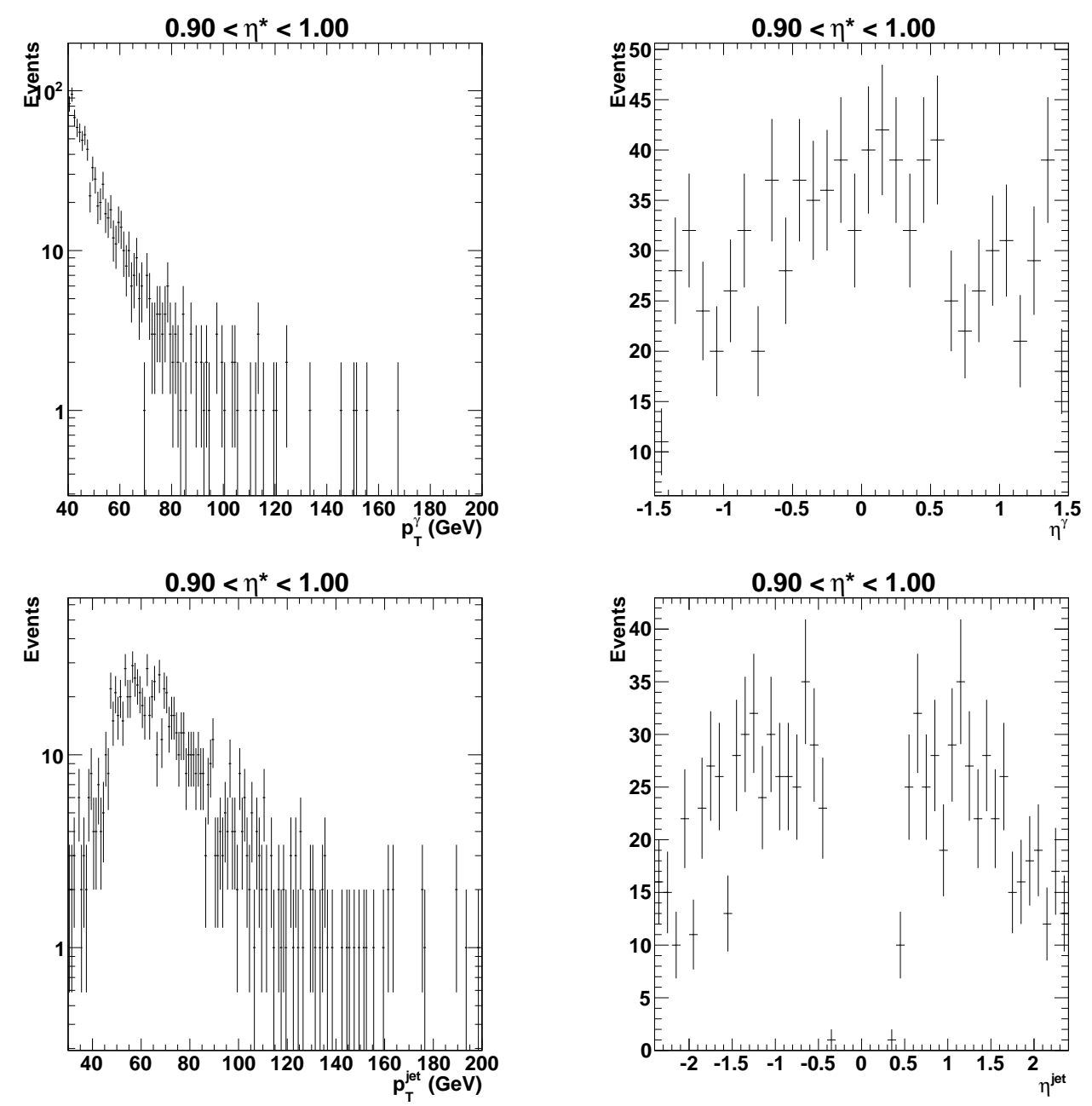

Figure A.10: The leading photon's $p_{T}$ and $\eta$ distributions (top) and the leading jet's $p_{T}$ and $\eta$ distributions on bottom for candidates with $0.9<\left|\eta^{*}\right|<1.0$. 

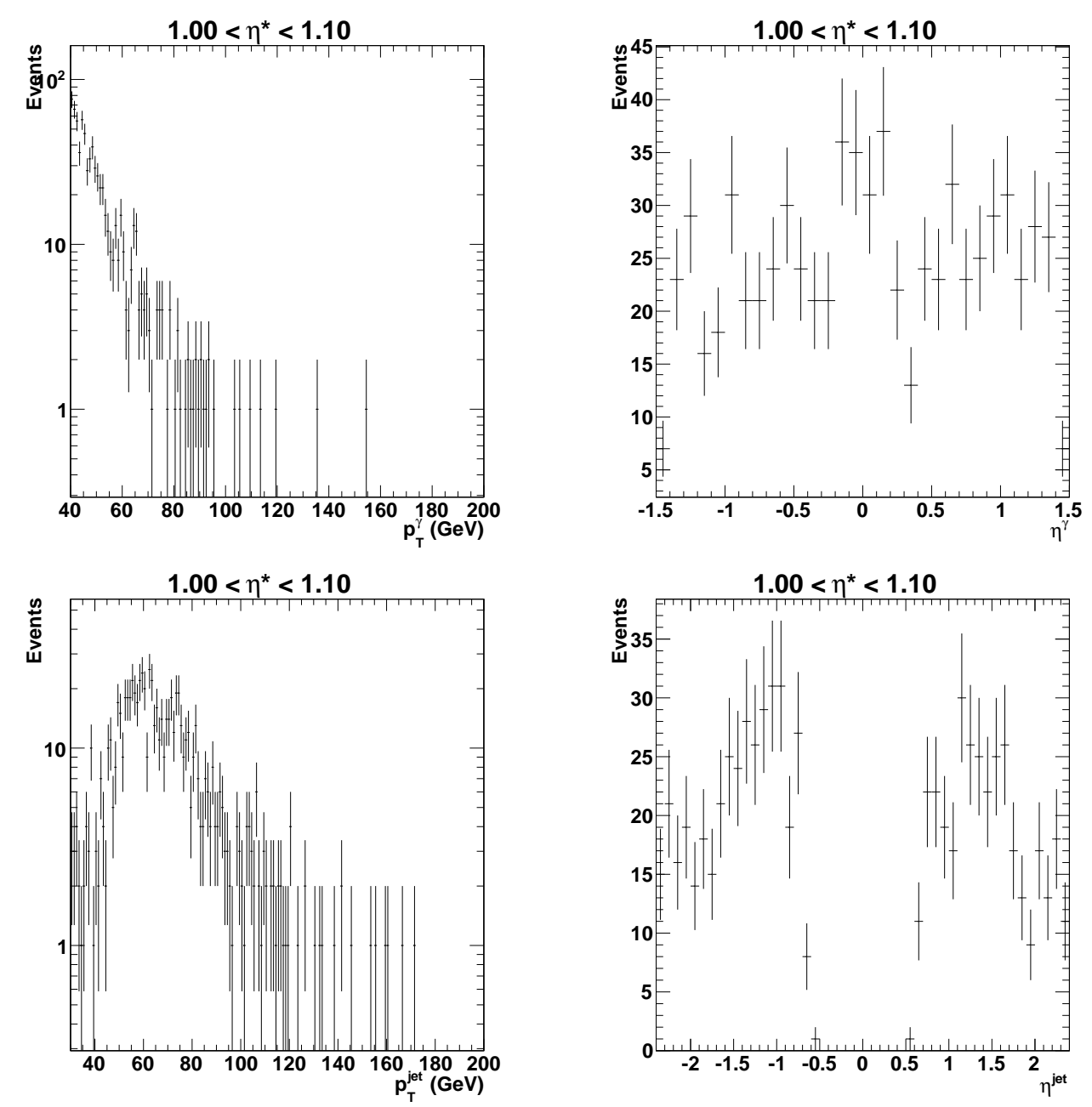

Figure A.11: The leading photon's $p_{T}$ and $\eta$ distributions (top) and the leading jet's $p_{T}$ and $\eta$ distributions on bottom for candidates with $1.0<\left|\eta^{*}\right|<1.1$. 

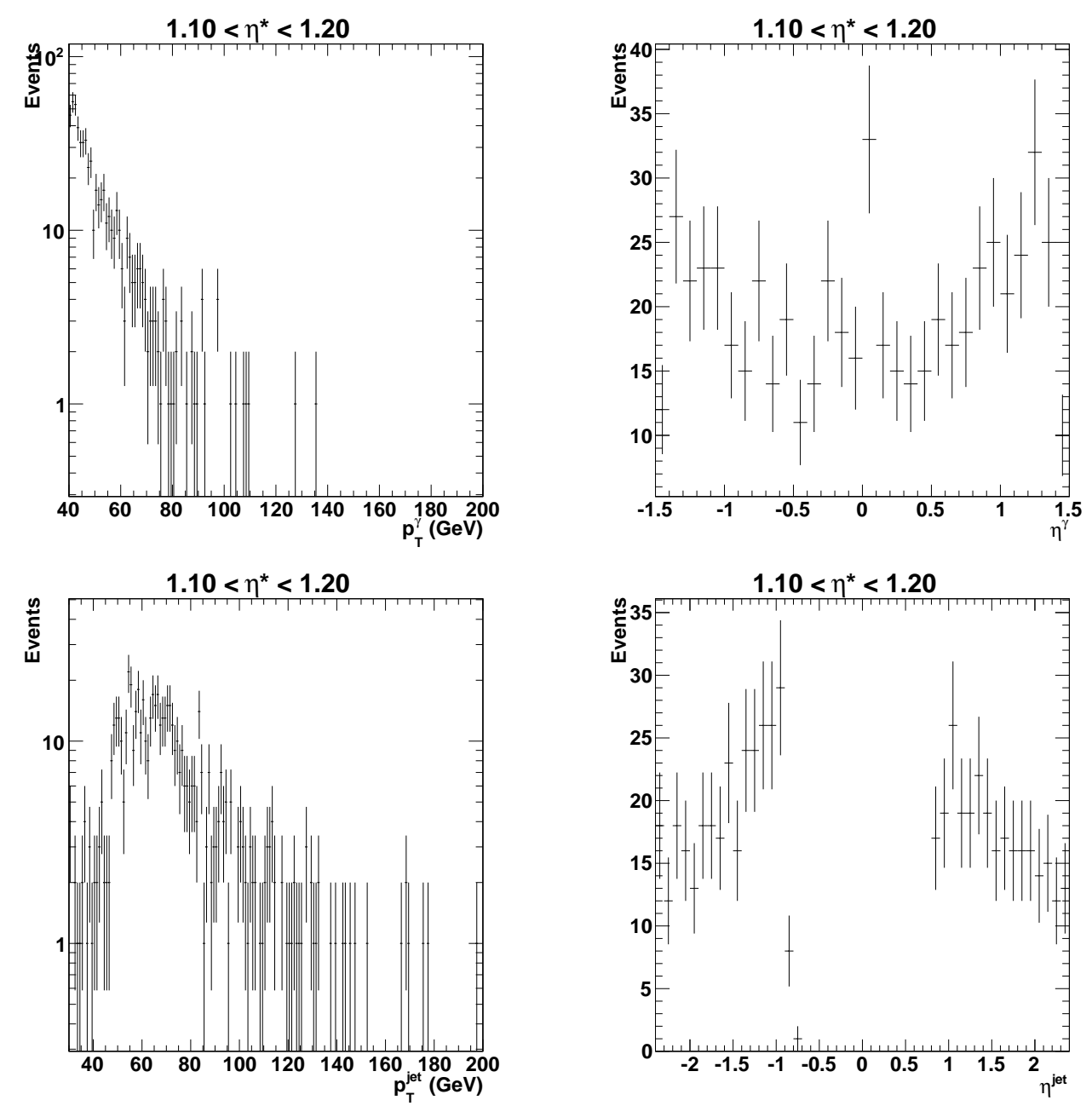

Figure A.12: The leading photon's $p_{T}$ and $\eta$ distributions (top) and the leading jet's $p_{T}$ and $\eta$ distributions on bottom for candidates with $1.1<\left|\eta^{*}\right|<1.2$. 

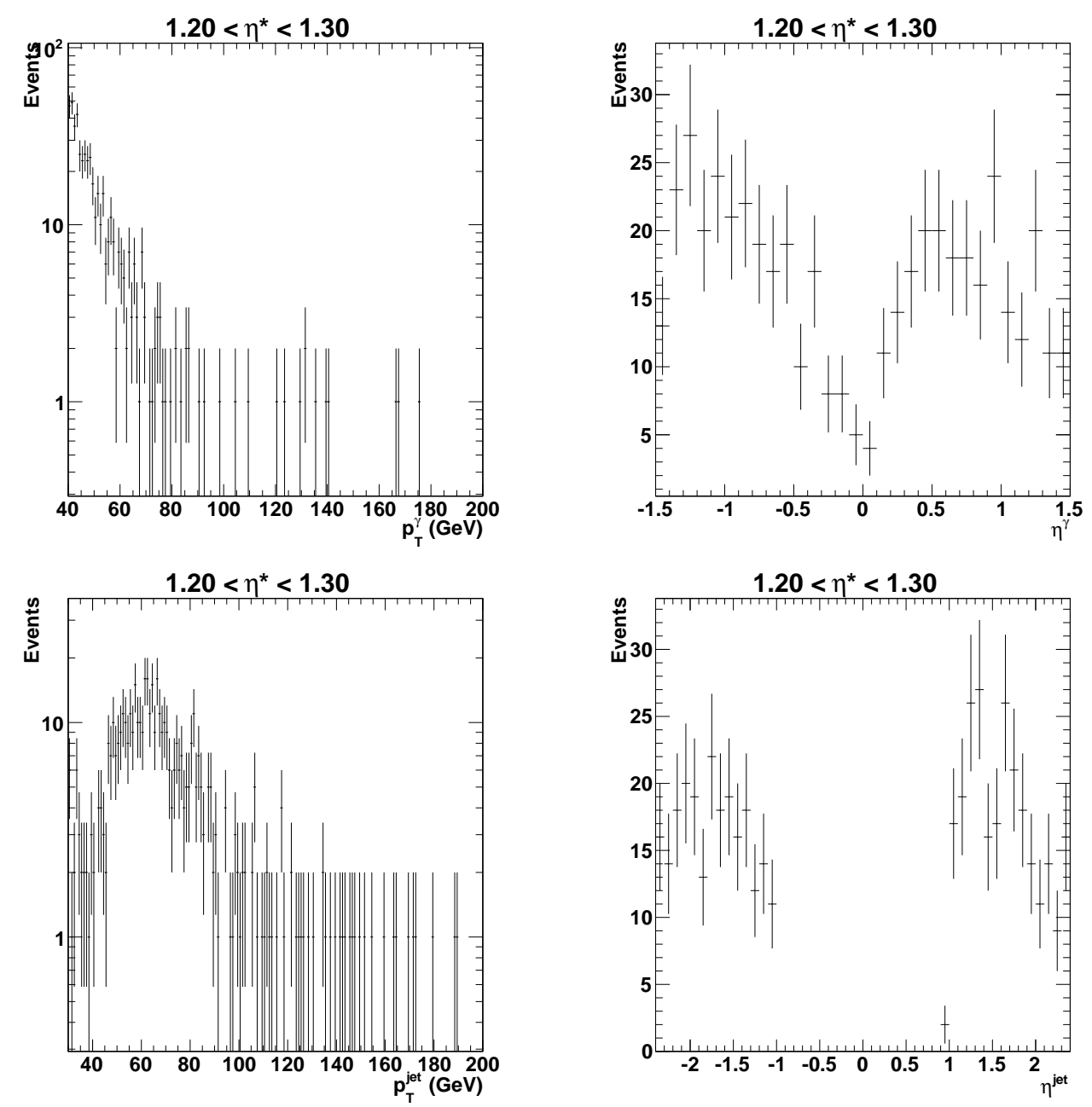

Figure A.13: The leading photon's $p_{T}$ and $\eta$ distributions (top) and the leading jet's $p_{T}$ and $\eta$ distributions on bottom for candidates with $1.2<\left|\eta^{*}\right|<1.3$. 

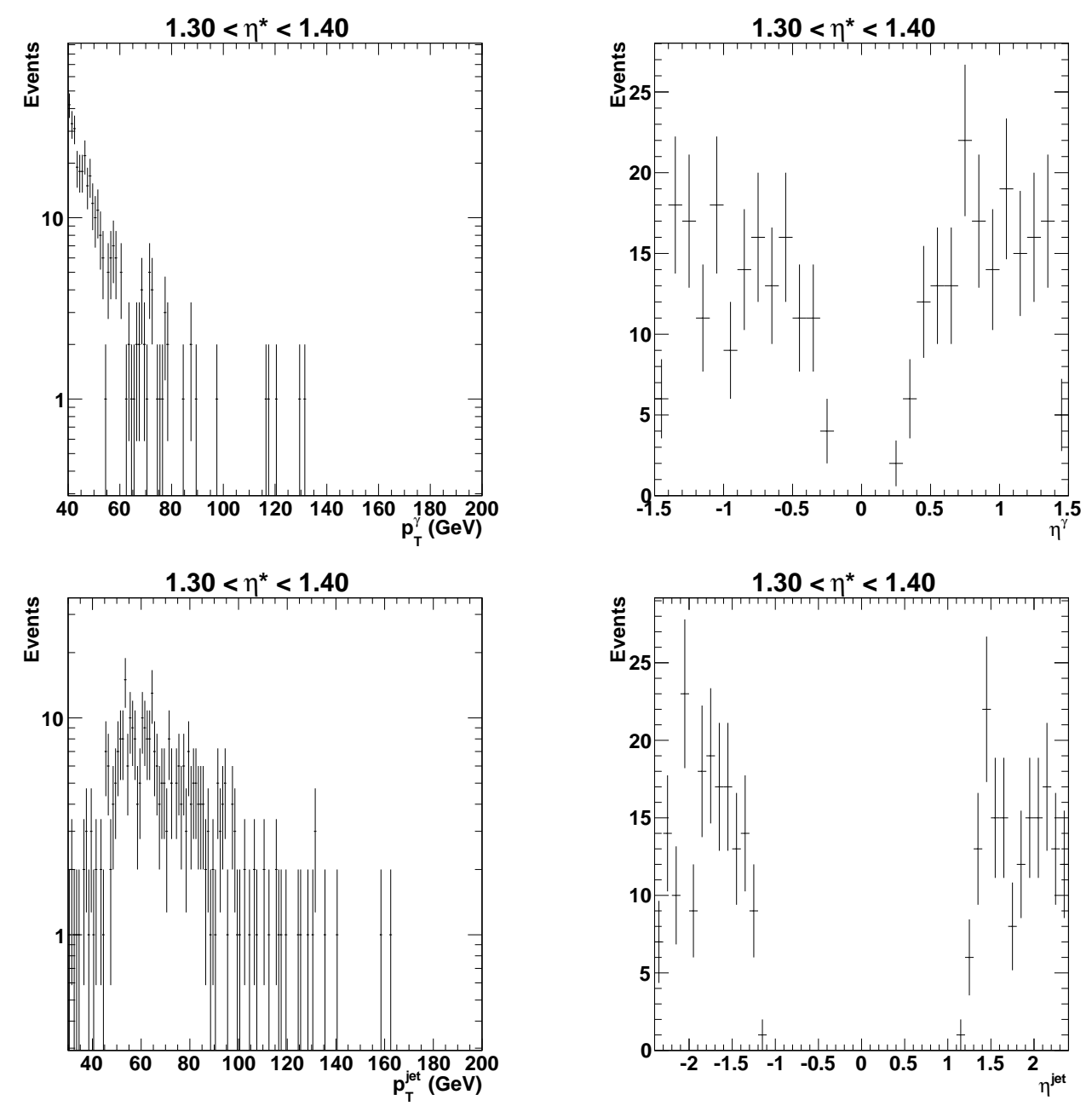

Figure A.14: The leading photon's $p_{T}$ and $\eta$ distributions (top) and the leading jet's $p_{T}$ and $\eta$ distributions on bottom for candidates with $1.3<\left|\eta^{*}\right|<1.4$. 


\section{APPENDIX B \\ PURITY EXPRESSION DERIVATION}

The purity is defined as the fraction of signal candidates in data:

$$
P=\frac{N_{s i g}}{N}
$$

In a template, the candidates in data, $N$, is a combination of the signal in which this analysis is focused (photons) and background (QCD jets). This is expressed as:

$$
N=N_{s i g}+N_{b k g}
$$

A boundary is placed on the template such that it effectively separates the background contribution from signal. The contribution of signal and background in data is now represented by the following expression:

$$
\epsilon_{d a t} N=\epsilon_{s i g} N_{s i g}+\epsilon_{b k g} N_{b k g}
$$

where $\epsilon_{d a t}, \epsilon_{s i g}$ and $\epsilon_{b k g}$ represent the fraction of candidates below the boundary in data, signal and background templates, respectively. Multiplying both sides of Eq.B.2 by $-\epsilon_{b k g}$ and summing Eqs. B.2 and B.3 shows that the last term cancels out, giving the following:

$$
\begin{gathered}
-\epsilon_{b k g} N=-\epsilon_{b k g} N_{s i g}-\epsilon_{b k g} N_{b k g} \\
\epsilon_{d a t} N=\epsilon_{s i g} N_{s i g}+\epsilon_{b k g N_{b k g}} \\
N\left(\epsilon_{d a t}-\epsilon_{b k g}\right)=N_{s i g}\left(\epsilon_{s i g}-b k g\right.
\end{gathered}
$$


Dividing both sides by $N$ gives the expression for purity given in Eq. B.1 gives the two-bin purity expression. Solving for $P$ gives the two-bin purity expression:

$$
\frac{\epsilon_{d a t}-\epsilon_{b k g}}{\epsilon_{s i g}-{ }_{b k g}}=P
$$




\section{APPENDIX C}

\section{TEMPLATE SHAPES}

The following distributions show the $\sigma_{i \eta i \eta}$ distribution as well as the area under that curve for signal, background and data for each bin in $\left|\eta^{*}\right|$.
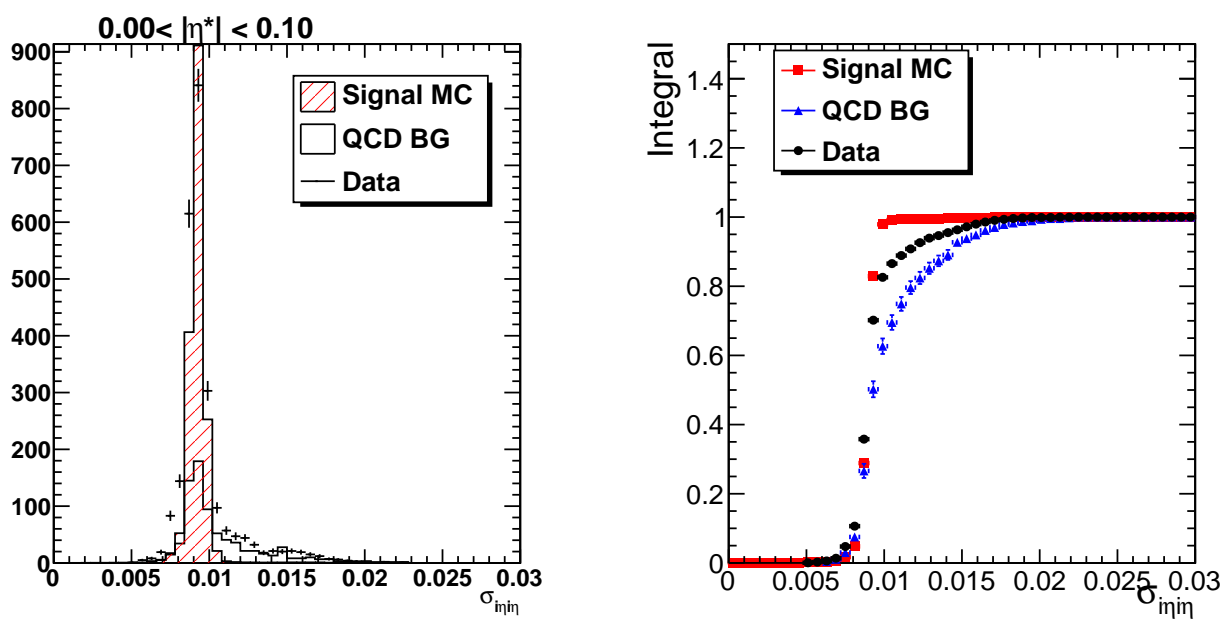

Figure C.1: The $\sigma_{i \eta i}$ distribution for photon candidates with $0.0<\left|\eta^{*}\right|<0.1$ for signal, background and data.
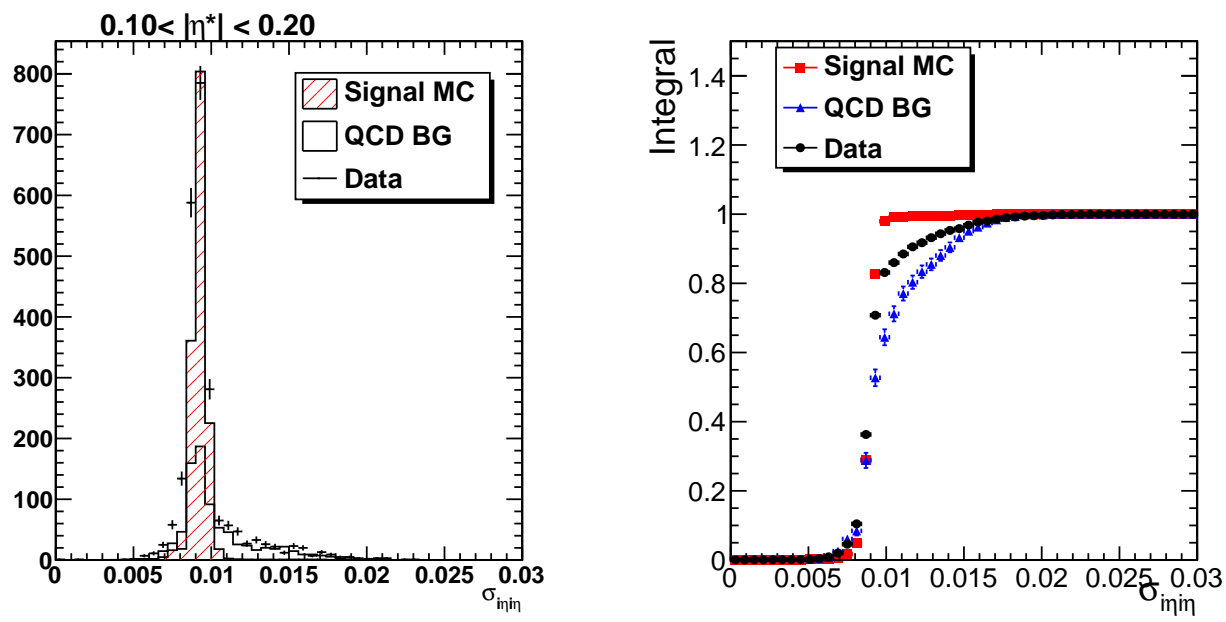

Figure C.2: The $\sigma_{i \eta i \eta}$ distribution for photon candidates with $0.1<\left|\eta^{*}\right|<0.2$ for signal, background and data. 

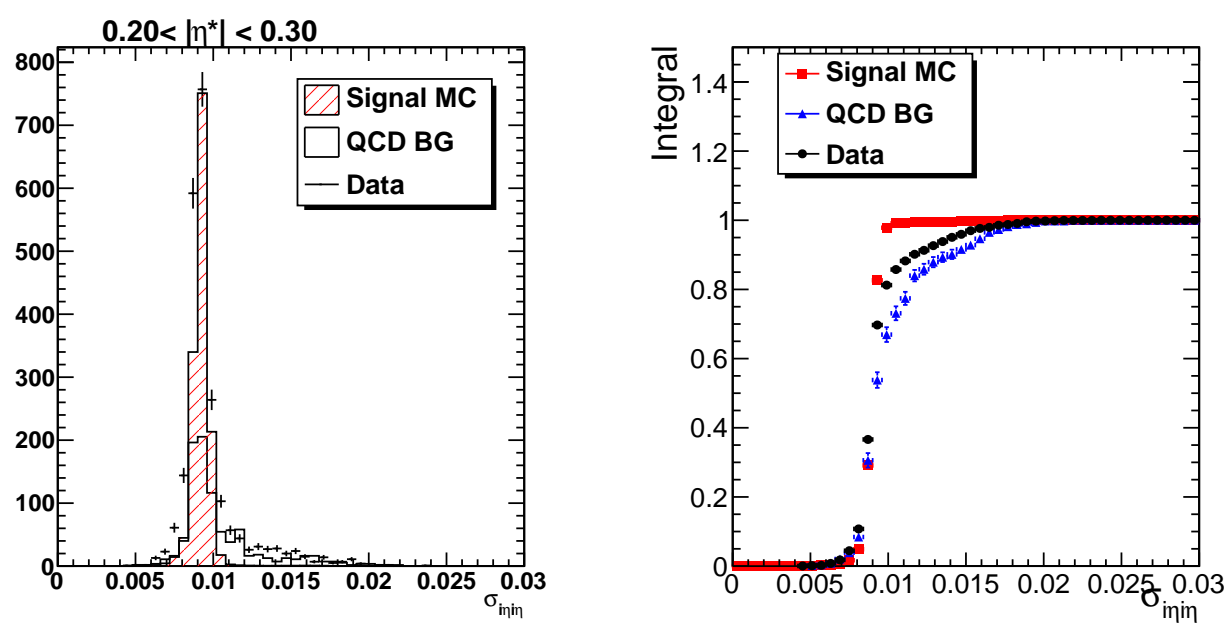

Figure C.3: The $\sigma_{i \eta i \eta}$ distribution for photon candidates with $0.2<\left|\eta^{*}\right|<0.3$ for signal, background and data.
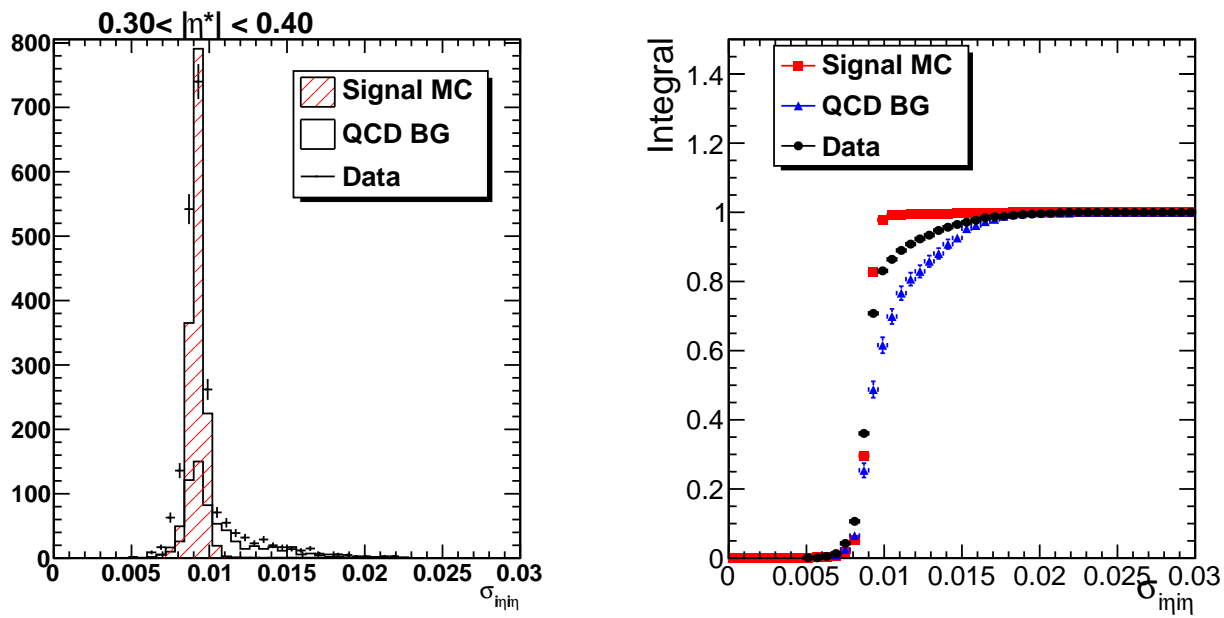

Figure C.4: The $\sigma_{i \eta i \eta}$ distribution for photon candidates with $0.3<\left|\eta^{*}\right|<0.4$ for signal, background and data. 

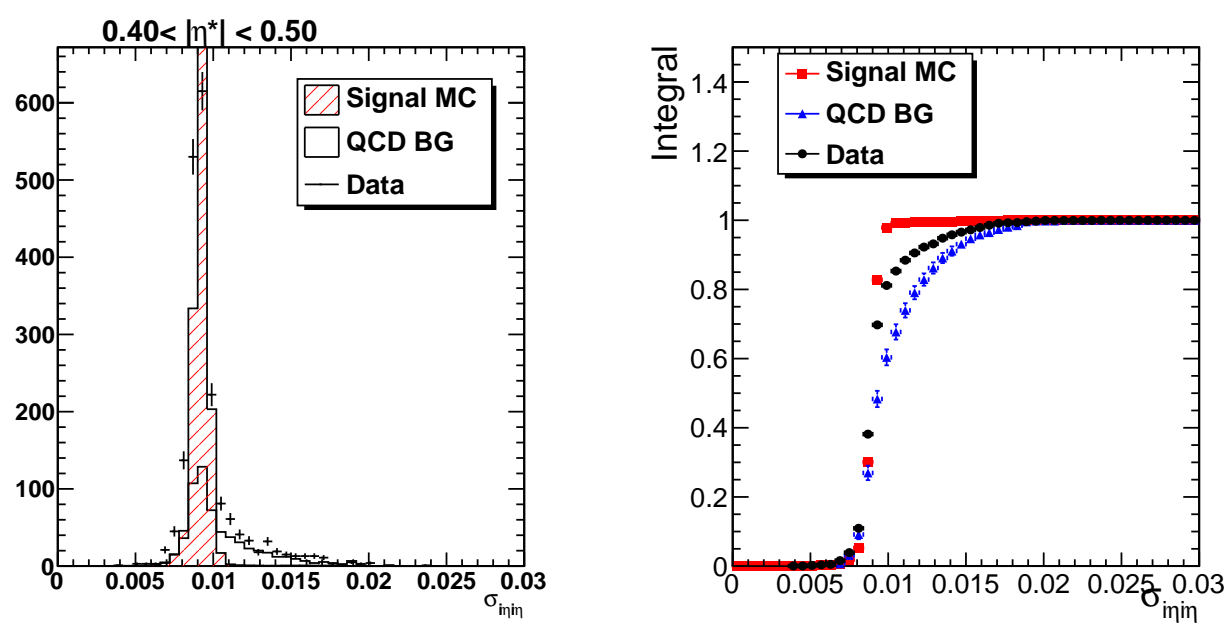

Figure C.5: The $\sigma_{i \eta i \eta}$ distribution for photon candidates with $0.4<\left|\eta^{*}\right|<0.5$ for signal, background and data.
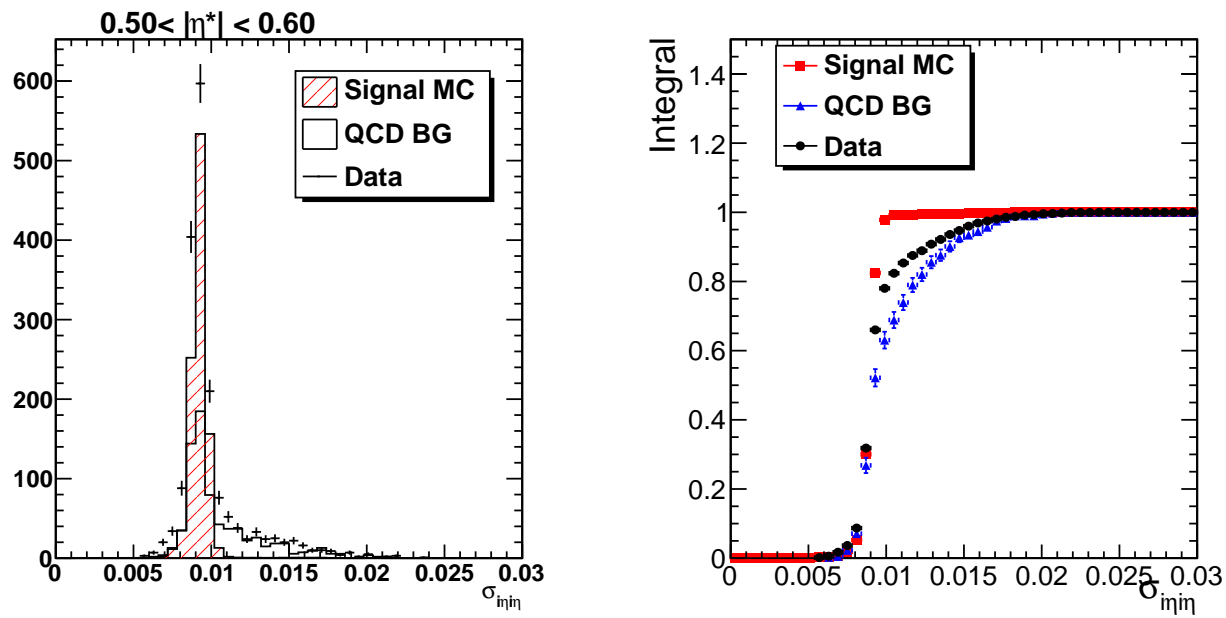

Figure C.6: The $\sigma_{i \eta i \eta}$ distribution for photon candidates with $0.5<\left|\eta^{*}\right|<0.6$ for signal, background and data. 

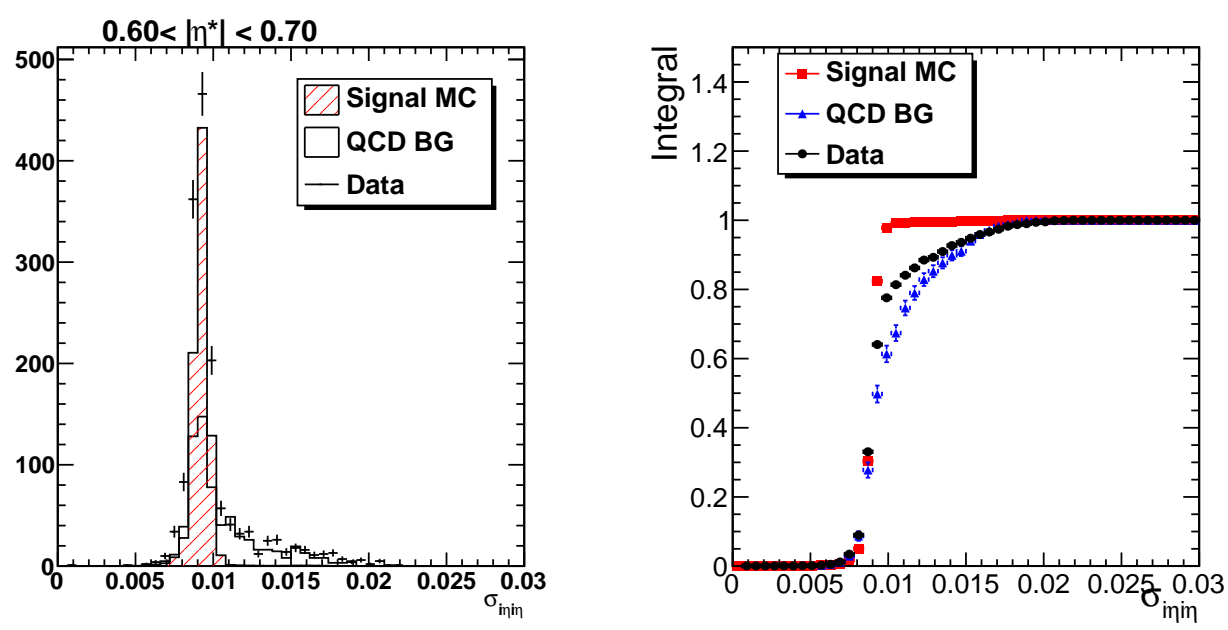

Figure C.7: The $\sigma_{i \eta i \eta}$ distribution for photon candidates with $0.6<\left|\eta^{*}\right|<0.7$ for signal, background and data.
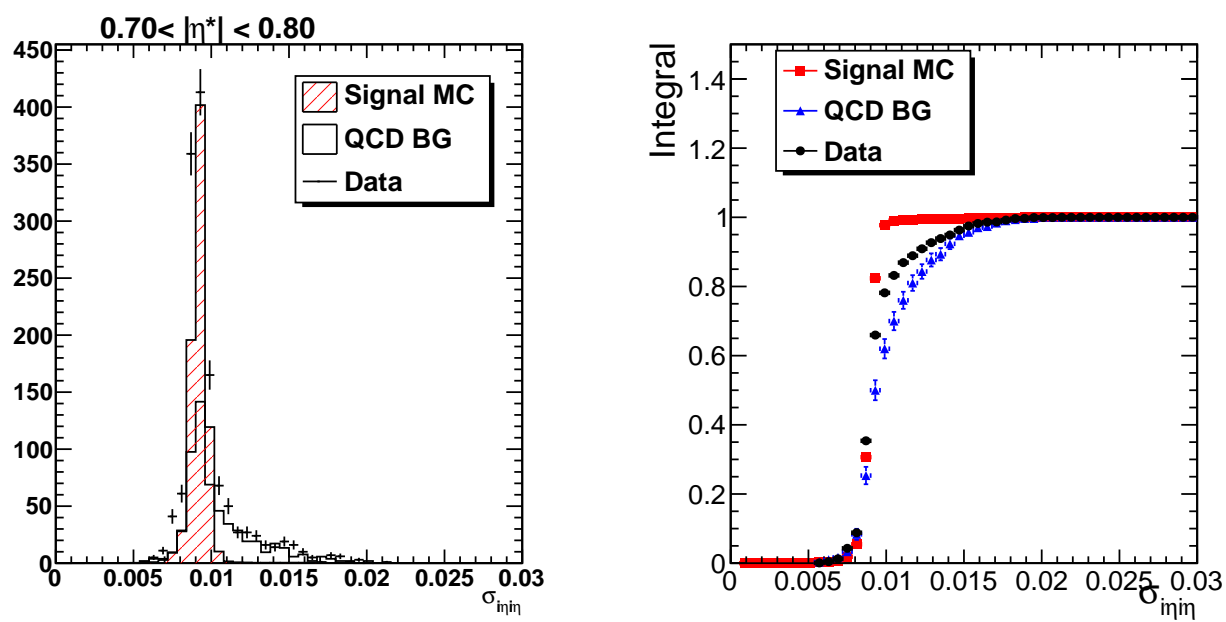

Figure C.8: The $\sigma_{i \eta i \eta}$ distribution for photon candidates with $0.7<\left|\eta^{*}\right|<0.8$ for signal, background and data. 

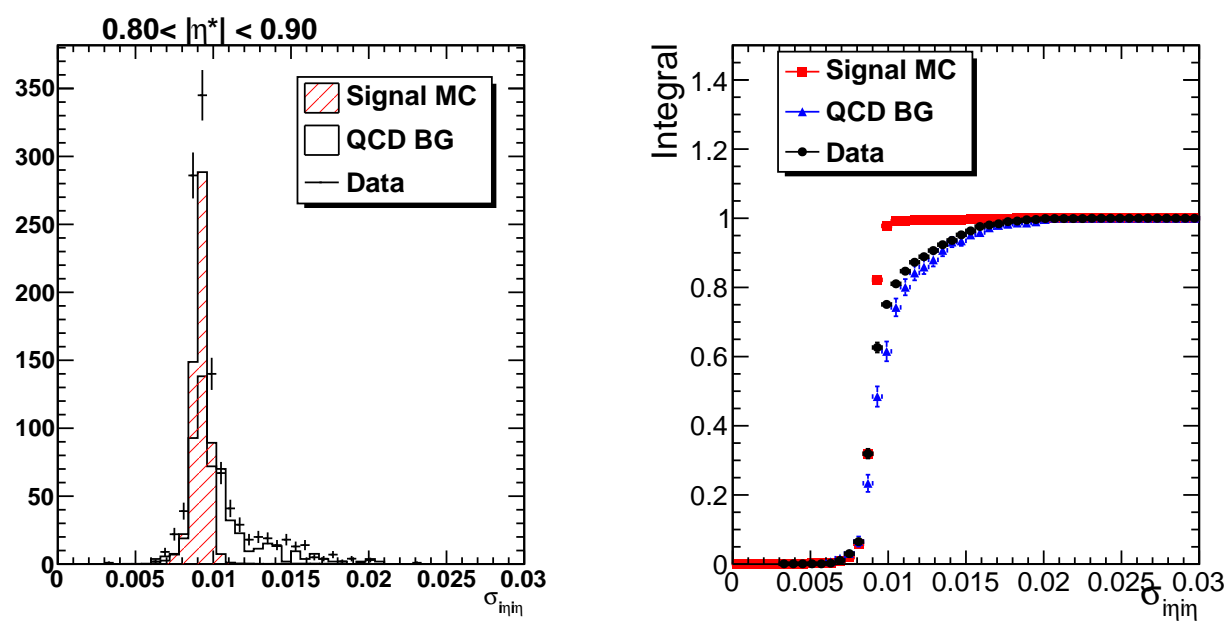

Figure C.9: The $\sigma_{i \eta i \eta}$ distribution for photon candidates with $0.8<\left|\eta^{*}\right|<0.9$ for signal, background and data.
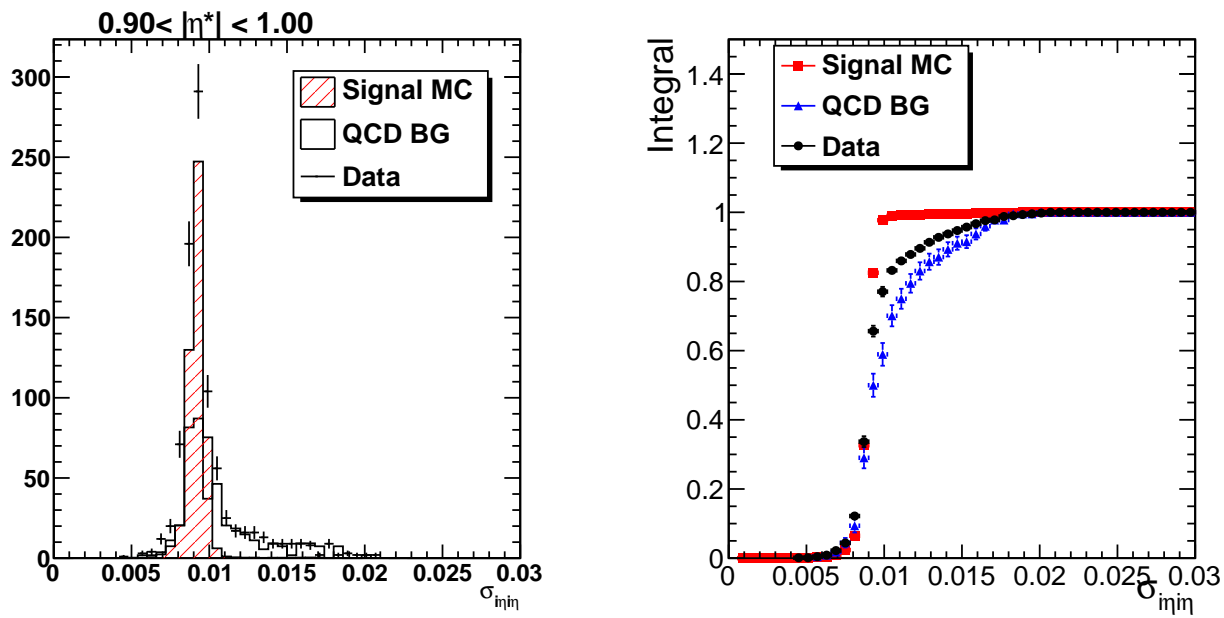

Figure C.10: The $\sigma_{i \eta i \eta}$ distribution for photon candidates with $0.9<\left|\eta^{*}\right|<1.0$ for signal, background and data. 

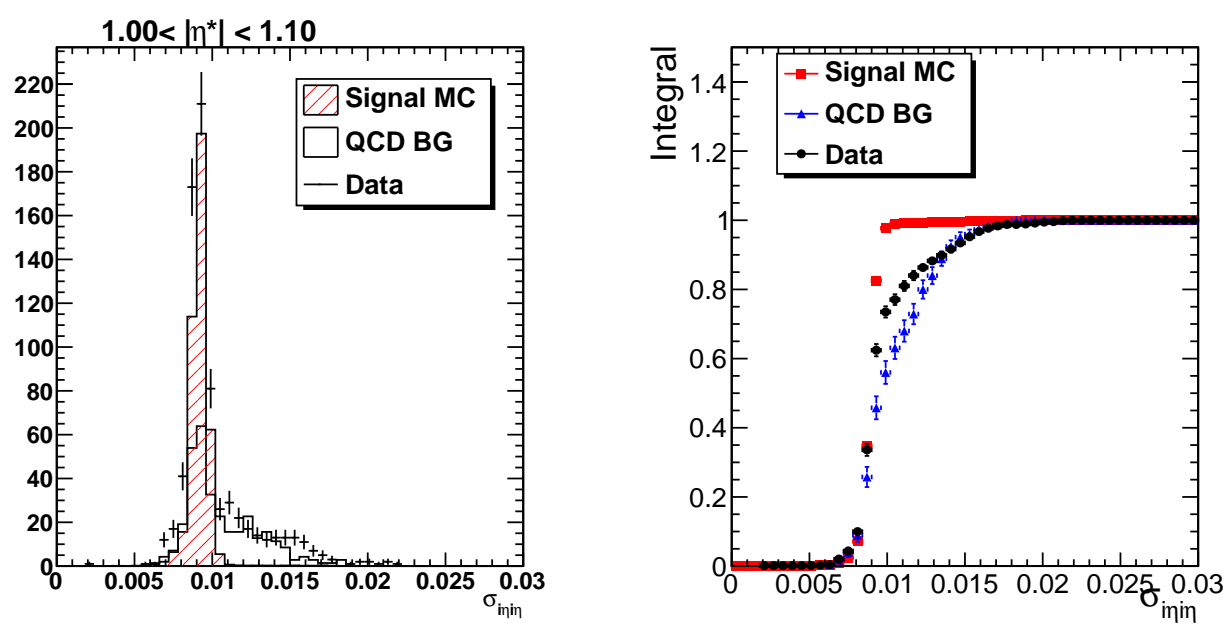

Figure C.11: The $\sigma_{i \eta i \eta}$ distribution for photon candidates with $1.0<\left|\eta^{*}\right|<1.1$ for signal, background and data.
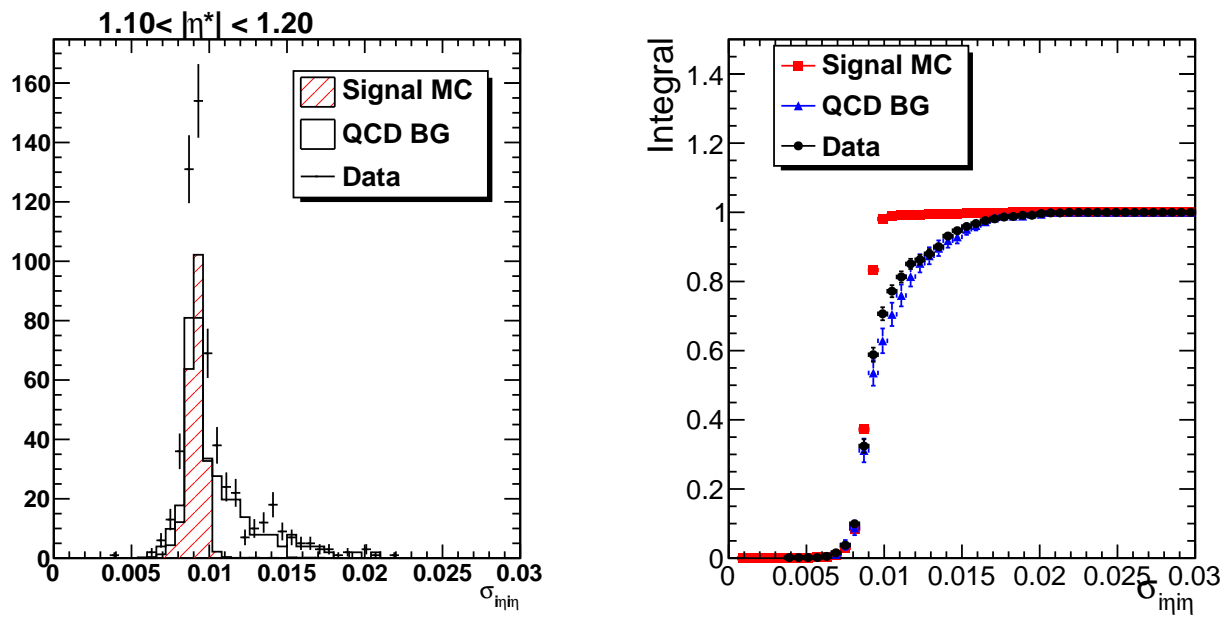

Figure C.12: The $\sigma_{i \eta i \eta}$ distribution for photon candidates with $1.1<\left|\eta^{*}\right|<1.2$ for signal, background and data. 

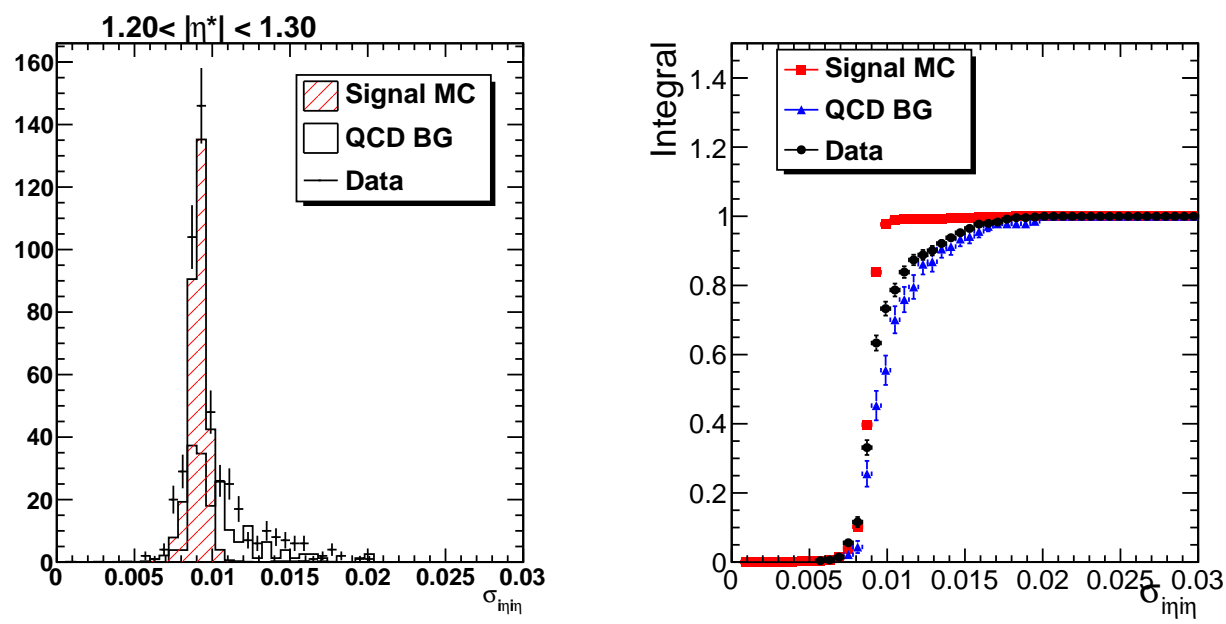

Figure C.13: The $\sigma_{i \eta i \eta}$ distribution for photon candidates with $1.2<\left|\eta^{*}\right|<1.3$ for signal, background and data.
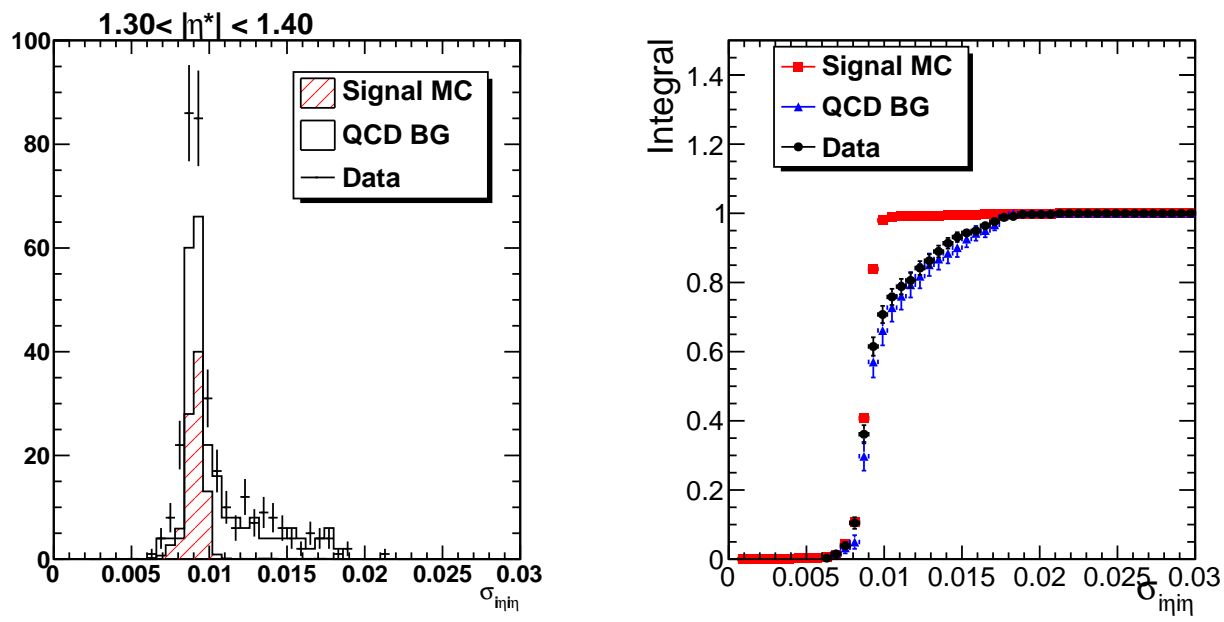

Figure C.14: The $\sigma_{i \eta i \eta}$ distribution for photon candidates with $1.3<\left|\eta^{*}\right|<1.4$ for signal, background and data. 


\section{APPENDIX D}

\section{A DATA-DRIVEN TECHNIQUE IN BACKGROUND MODELING}

The following plots show the $\sigma_{i \eta i \eta}$ distribution and the integral under the $\sigma_{i \eta i \eta}$ distribution in each bin in $\left|\eta^{*}\right|$ for the three background templates compared in order to validate the data-driven technique used to model the expected background in this analysis: QCD MC selected using photon identification cuts, QCD MC selected in the side-band region and data selected in the side-band region. The integral distributions for signal and data are also included in order to show that all three background integrals fall below the data integral while the signal integrals are above both the data and background integrals, as expected.
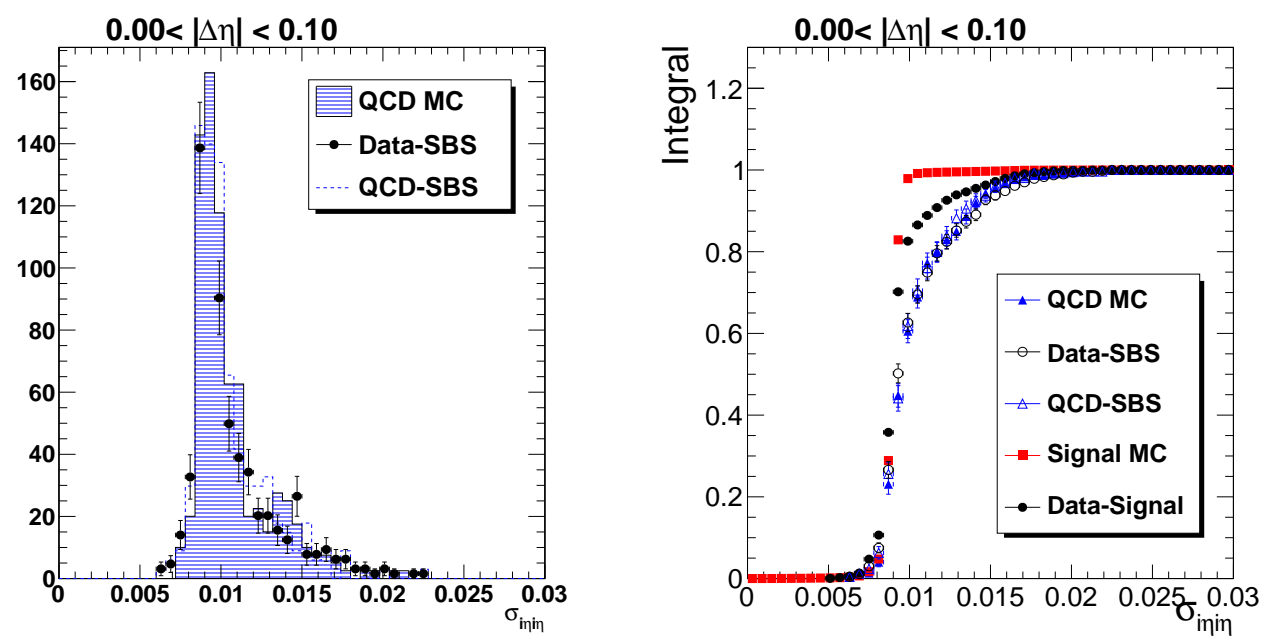

Figure D.1: The $\sigma_{i \eta i \eta}$ distribution for photon candidates with $0.0<\left|\eta^{*}\right|<0.1$ for the various background shapes used in this analysis. 

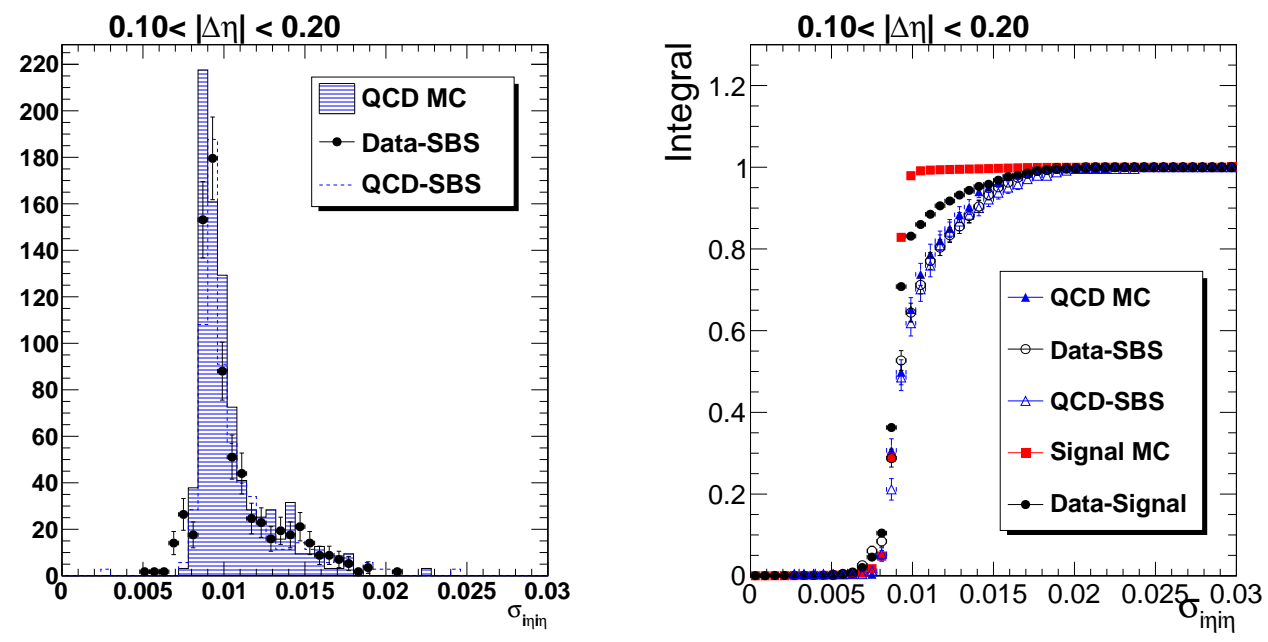

Figure D.2: The $\sigma_{i \eta i \eta}$ distribution for photon candidates with $0.1<\left|\eta^{*}\right|<0.2$ for the various background shapes used in this analysis.
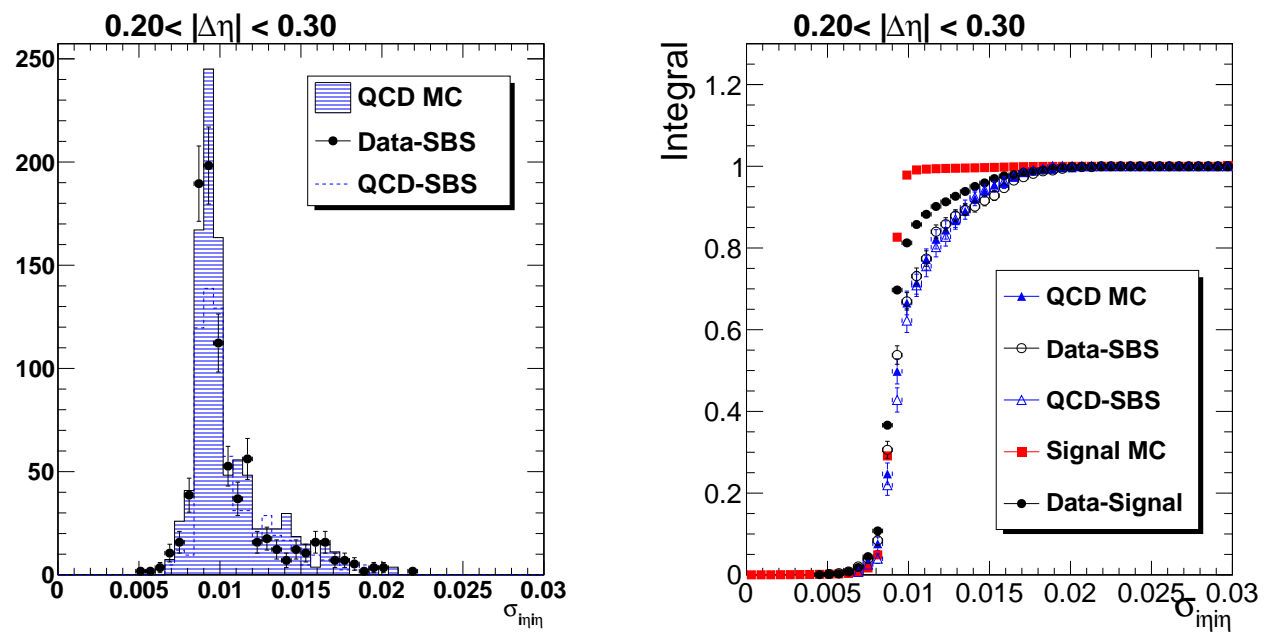

Figure D.3: The $\sigma_{i \eta i \eta}$ distribution for photon candidates with $0.2<\left|\eta^{*}\right|<0.3$ for the various background shapes used in this analysis. 

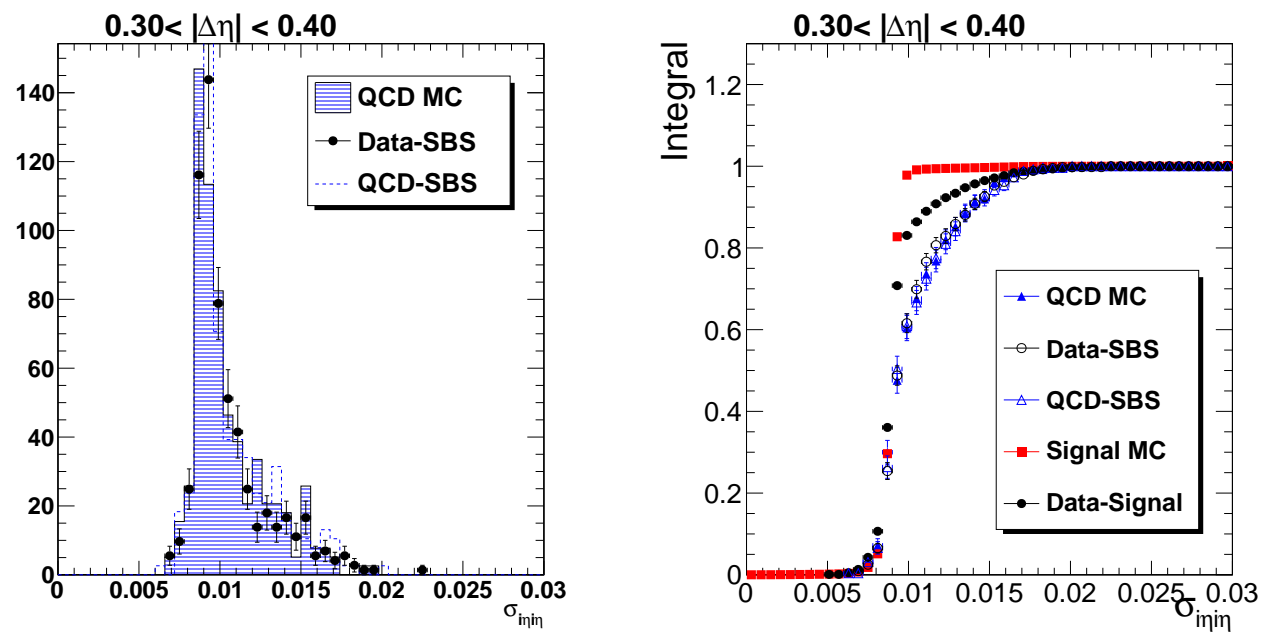

Figure D.4: The $\sigma_{i \eta i \eta}$ distribution for photon candidates with $0.3<\left|\eta^{*}\right|<0.4$ for the various background shapes used in this analysis.
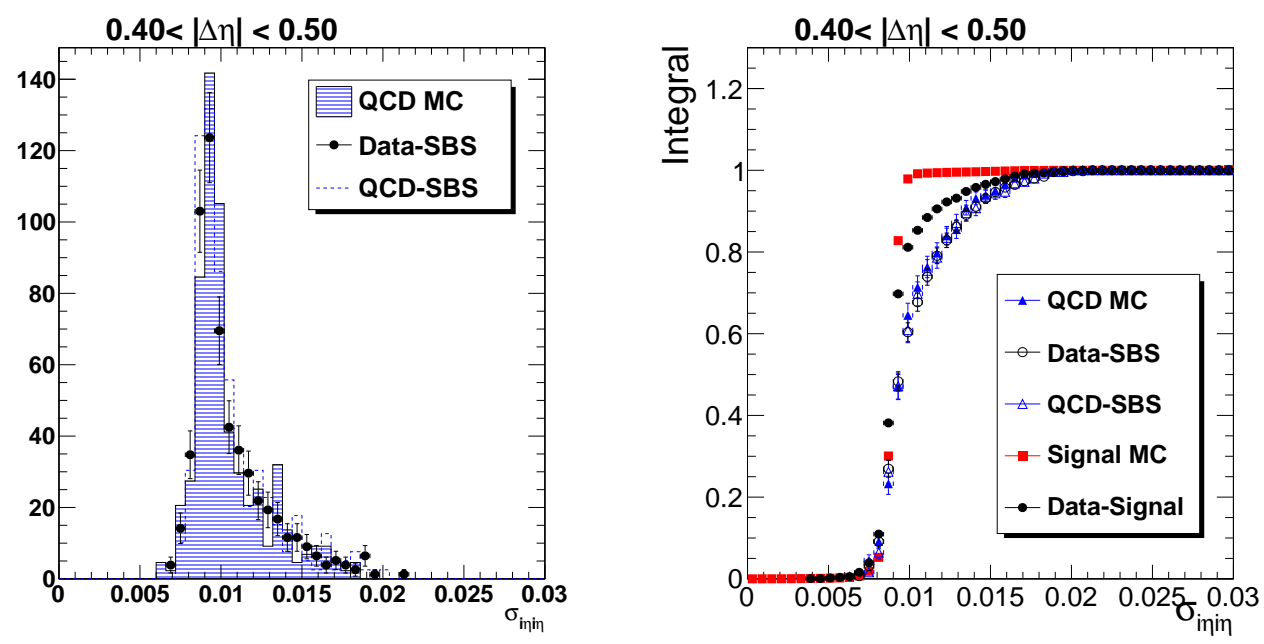

Figure D.5: The $\sigma_{i \eta i \eta}$ distribution for photon candidates with $0.4<\left|\eta^{*}\right|<0.5$ for the various background shapes used in this analysis. 

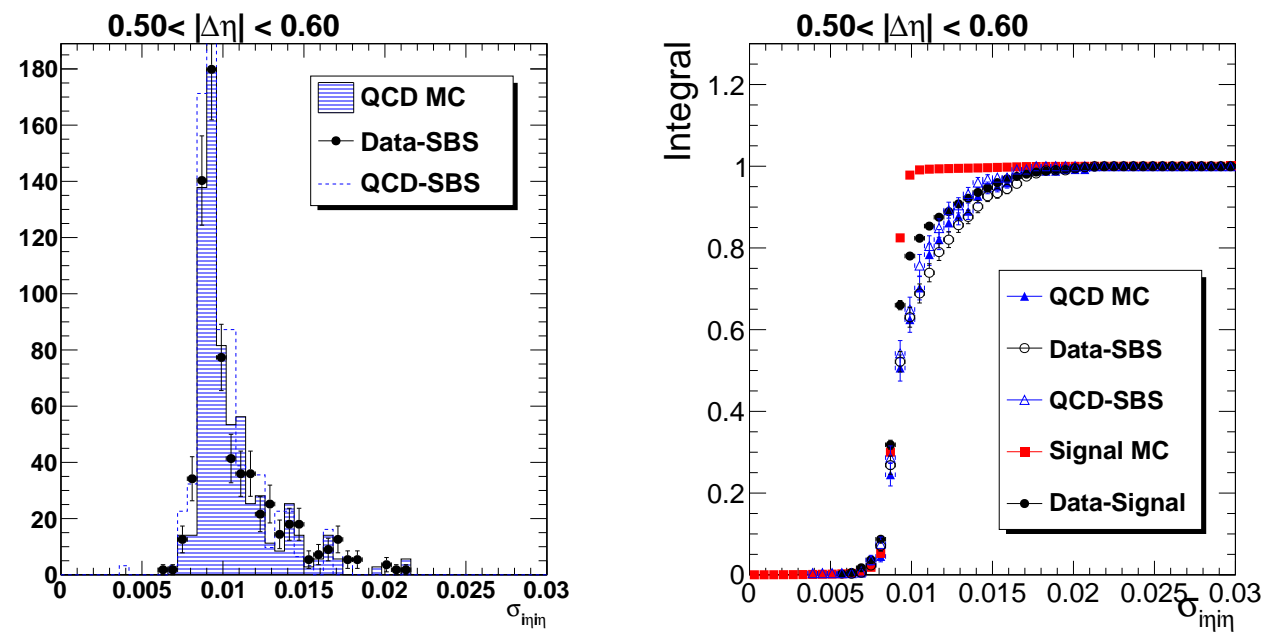

Figure D.6: The $\sigma_{i \eta i \eta}$ distribution for photon candidates with $0.5<\left|\eta^{*}\right|<0.6$ for the various background shapes used in this analysis.
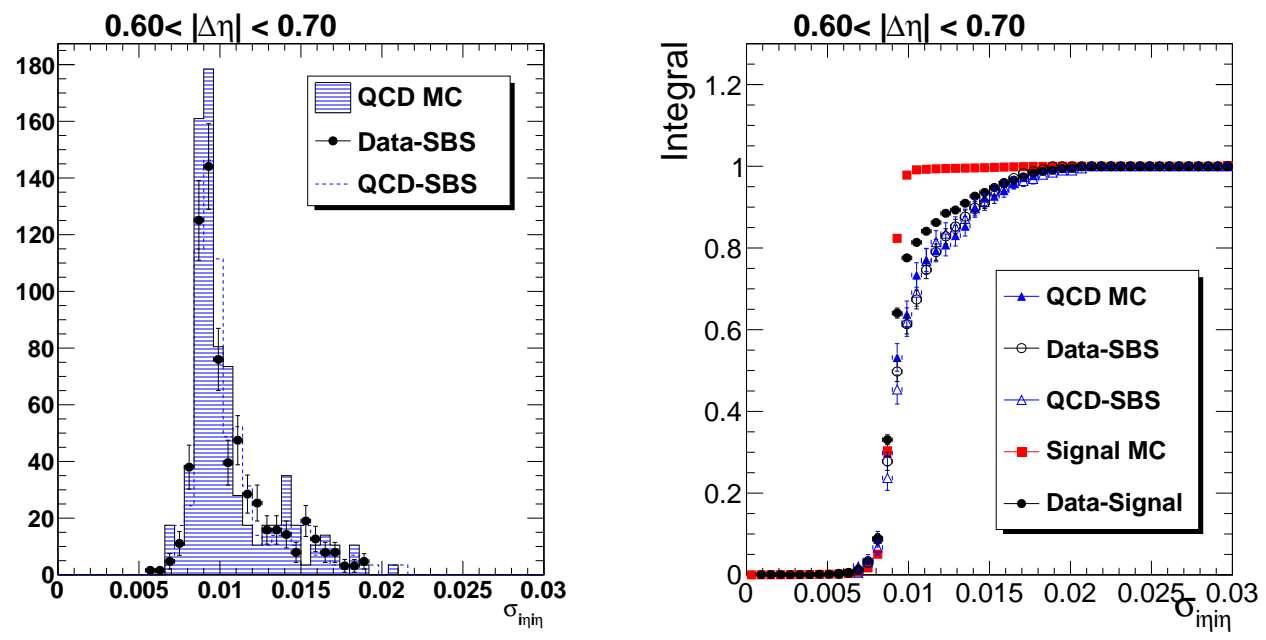

Figure D.7: The $\sigma_{i \eta i \eta}$ distribution for photon candidates with $0.6<\left|\eta^{*}\right|<0.7$ for the various background shapes used in this analysis. 

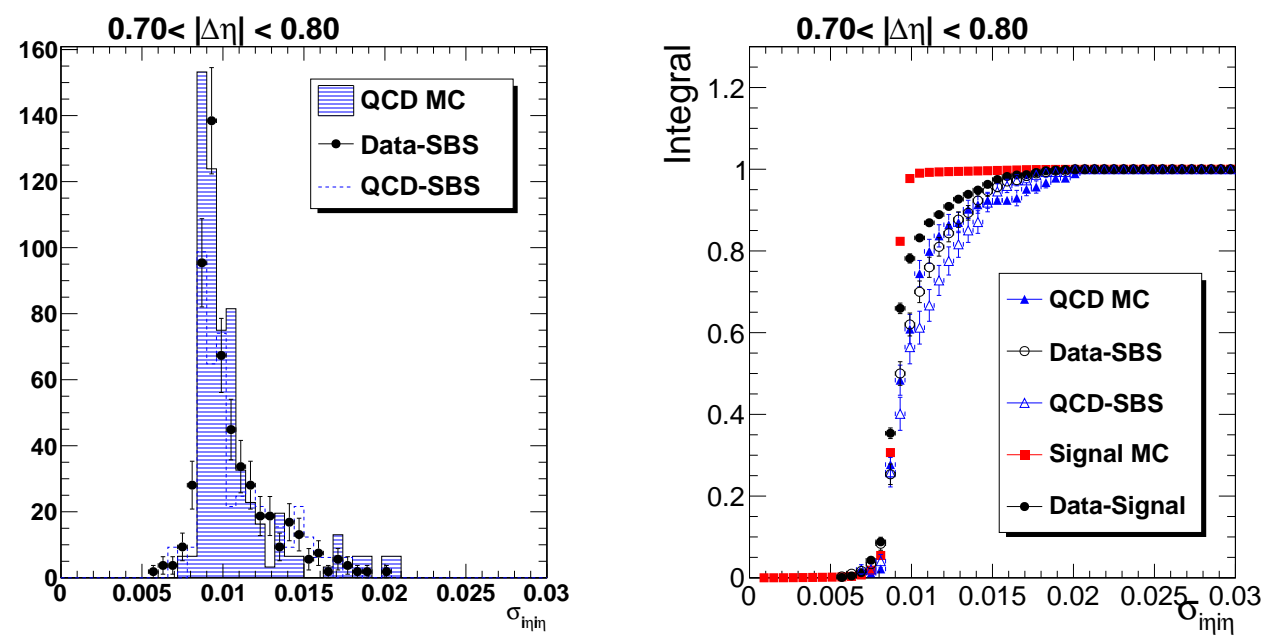

Figure D.8: The $\sigma_{i \eta i \eta}$ distribution for photon candidates with $0.7<\left|\eta^{*}\right|<0.8$ for the various background shapes used in this analysis.
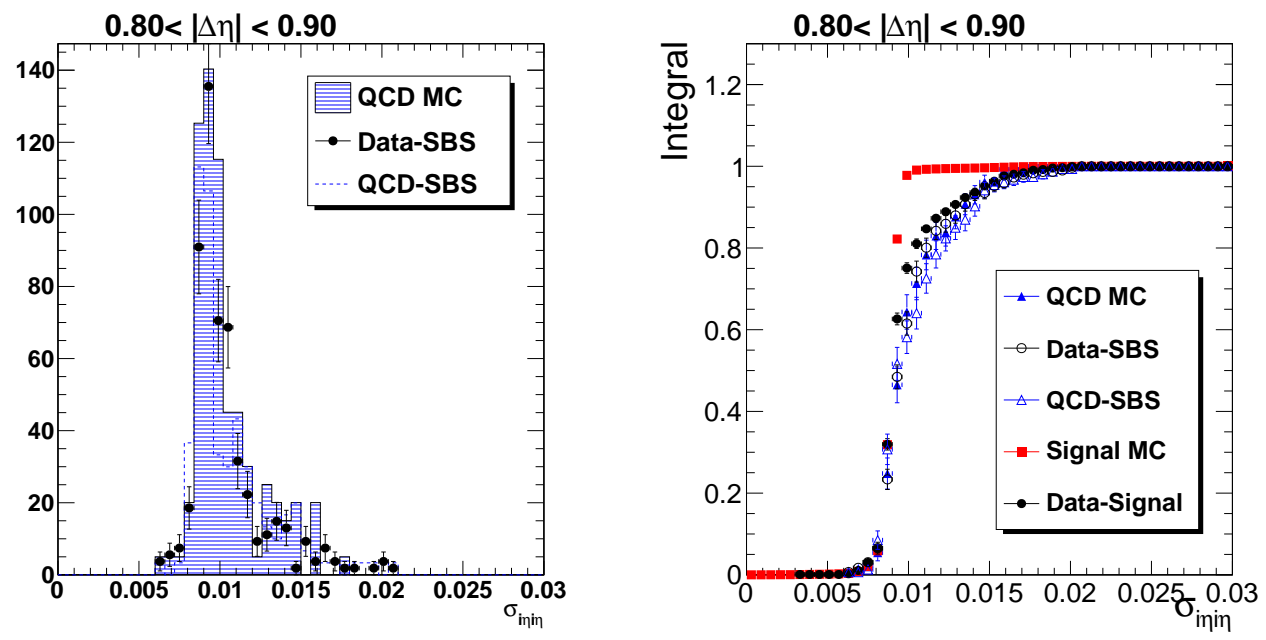

Figure D.9: The $\sigma_{i \eta i \eta}$ distribution for photon candidates with $0.8<\left|\eta^{*}\right|<0.9$ for the various background shapes used in this analysis. 

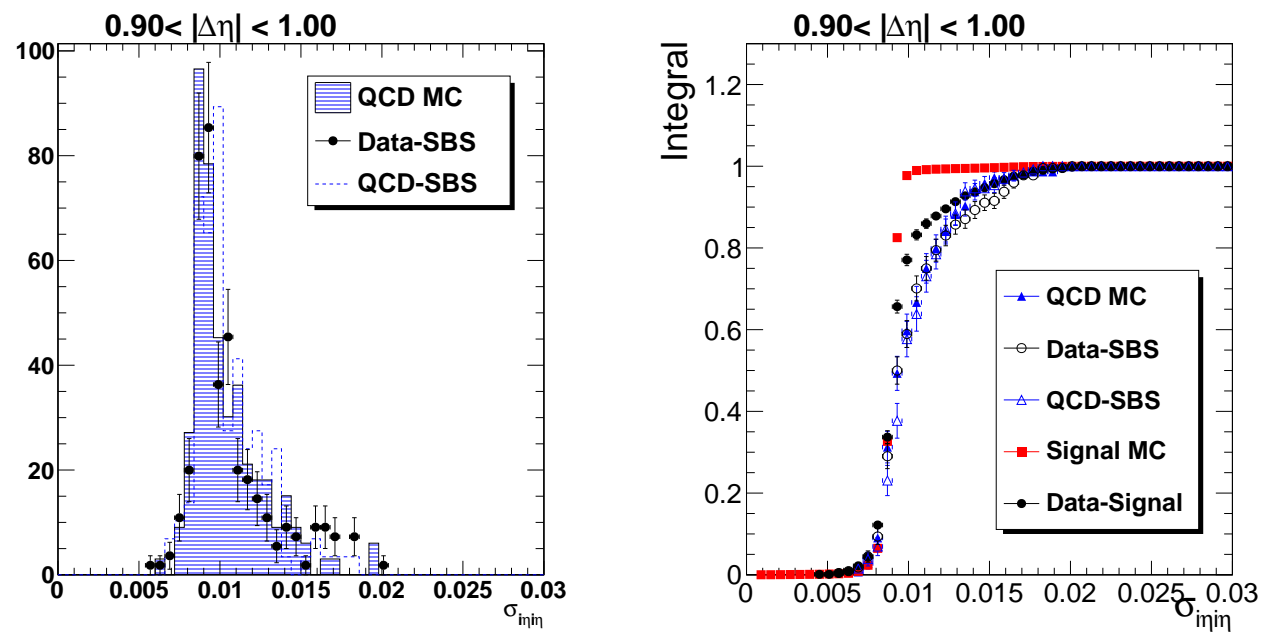

Figure D.10: The $\sigma_{i \eta \eta \eta}$ distribution for photon candidates with $0.9<\left|\eta^{*}\right|<1.0$ for the various background shapes used in this analysis.
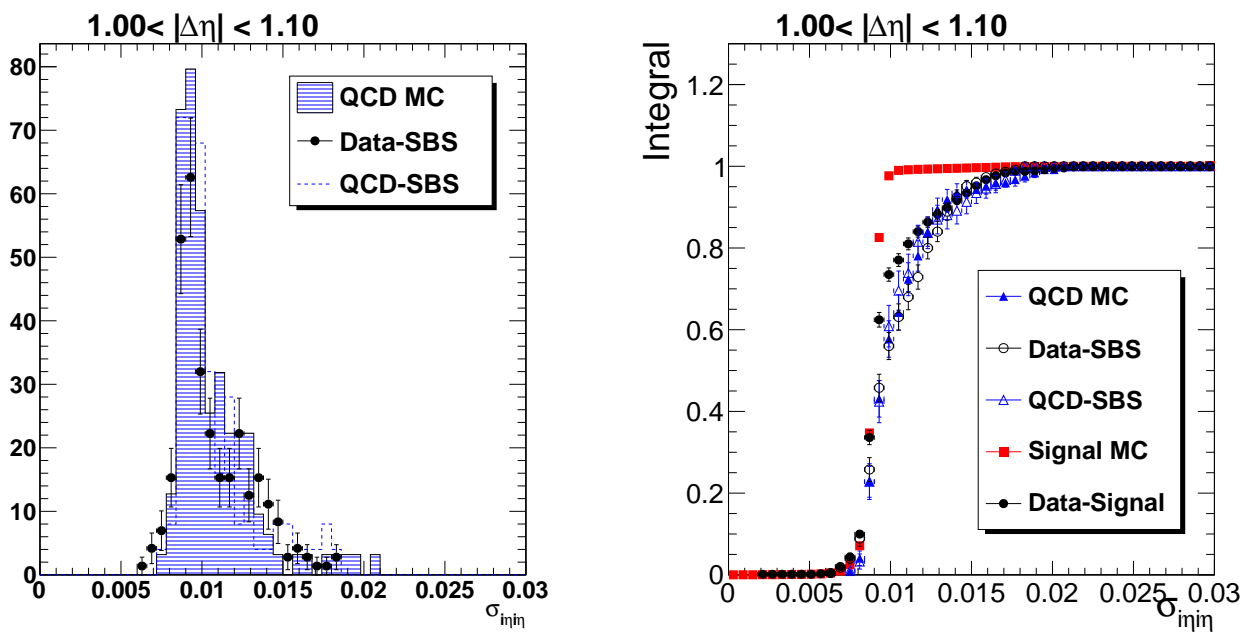

Figure D.11: The $\sigma_{i \eta i \eta}$ distribution for photon candidates with $1.0<\left|\eta^{*}\right|<1.1$ for the various background shapes used in this analysis. 

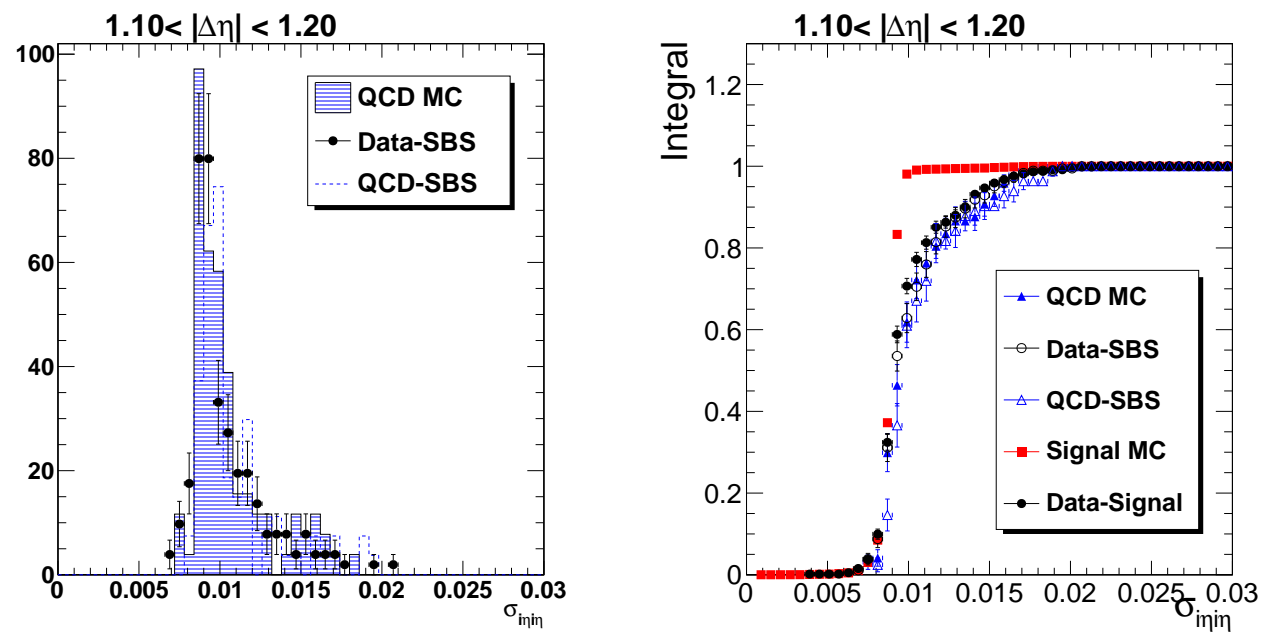

Figure D.12: The $\sigma_{i \eta \eta \eta}$ distribution for photon candidates with $1.1<\left|\eta^{*}\right|<1.2$ for the various background shapes used in this analysis.
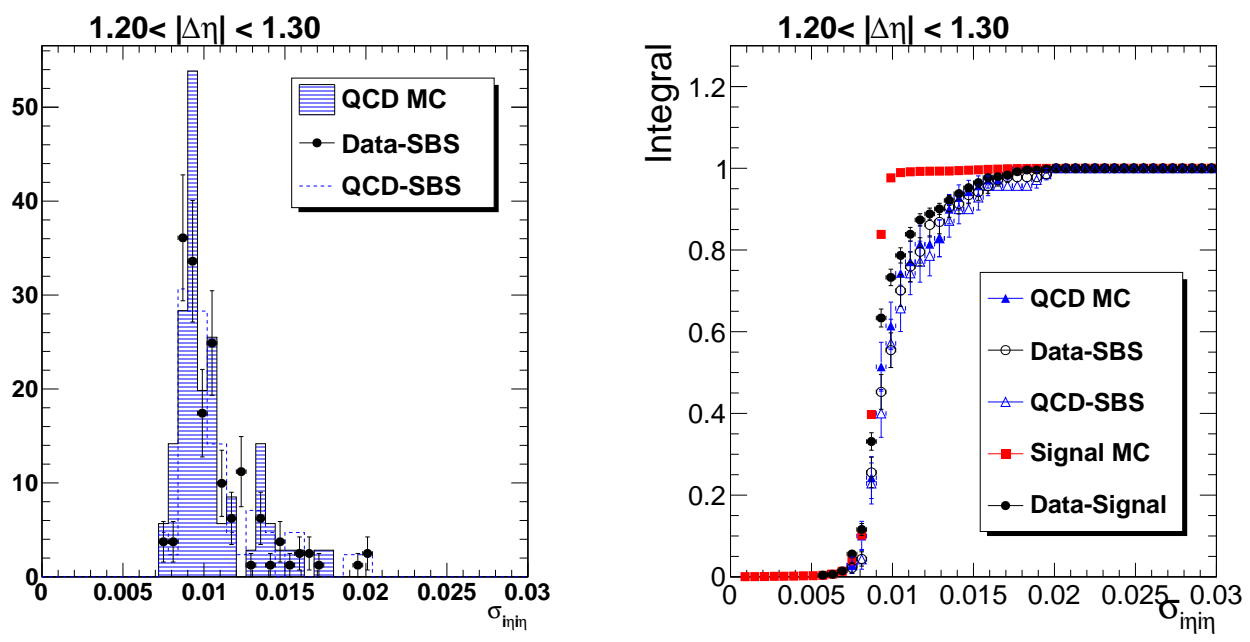

Figure D.13: The $\sigma_{i \eta i \eta}$ distribution for photon candidates with $1.2<\left|\eta^{*}\right|<1.3$ for the various background shapes used in this analysis. 

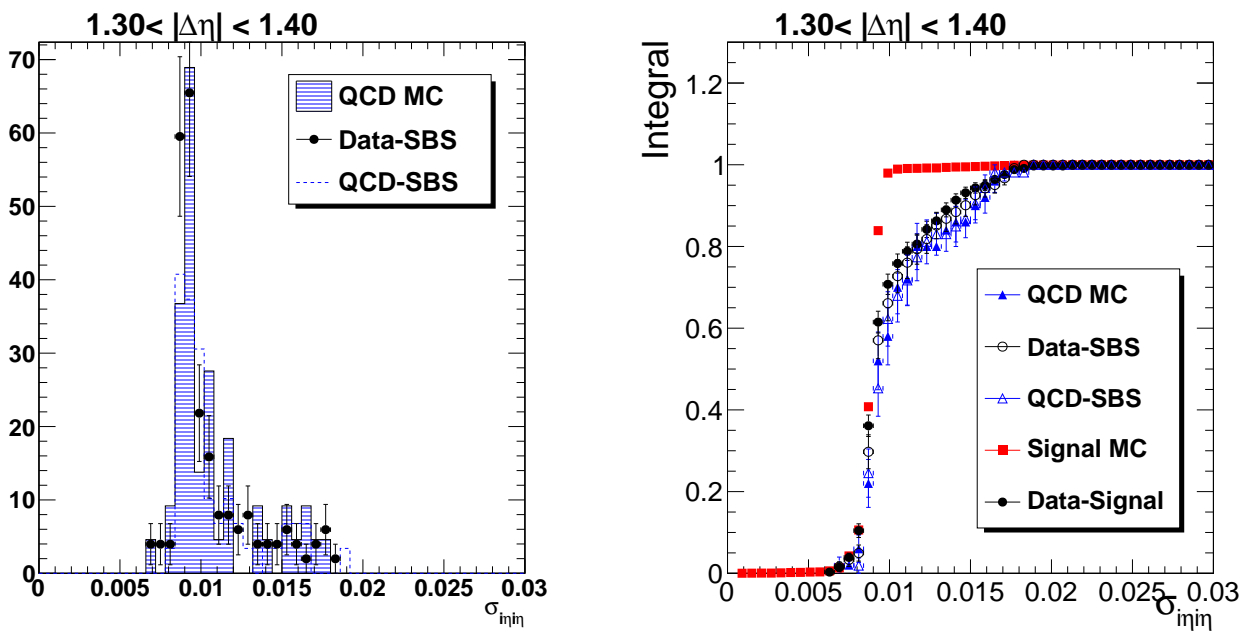

Figure D.14: The $\sigma_{i \eta i \eta}$ distribution for photon candidates with $1.3<\left|\eta^{*}\right|<1.4$ for the various background shapes used in this analysis. 


\section{APPENDIX E \\ COMPARISONS BETWEEN THE TWO-BIN METHOD AND 'ABCD' METHOD}

The 'ABCD' method of background estimation considers the number of candidates present in a 2-dimensional distribution of uncorrelated variables in regions labeled A, $\mathrm{B}, \mathrm{C}$ and $\mathrm{D}$ represented in the following expression:

$$
\frac{N_{A}}{N_{C}}=\frac{N_{B}}{N_{D}}
$$

Fig. E.1 shows such a 2D distribution with the y-axis labeled as $\sigma_{i \eta i}$ and the x-axis labeled as $\mathrm{ISO}_{T R K}$. Photon identification dictates that photon candidates are selected with $\mathrm{IsO}_{T R K}<2 \mathrm{GeV}$. Therefore, the remaining candidates in data with Iso $_{T R K}>2$ $\mathrm{GeV}$ will not qualify as a good candidate. In addition, the discriminant variable $\sigma_{i \eta i \eta}$ depicts photon candidates to have a smaller shower shape than background, as was shown in Fig. 5.1. Photon candidates in data will populate region A, since it has candidates with $\sigma_{i \eta i}$ below the maximum allowed value as well as $\operatorname{Iso}_{T R K}<2 \mathrm{GeV}$ as per photon identification, though there will also be background contributions in this region such that $A=N_{A}^{s i g}+N_{A}^{b k g}$, while the remaining regions $\mathrm{B}, \mathrm{C}$ and $\mathrm{D}$ are assumed to be filled with background (i.e. $B=N_{B}^{b k g}, C=N_{C}^{b k g}$ and $D=N_{D}^{b k g}$ ).

Using Eq. E.1 the background contribution can be estimated in region A:

$$
N_{A}^{b k g}=\frac{B C}{D}
$$

The signal present is then:

$$
S=\frac{A D-\frac{B C}{D}}{D}
$$




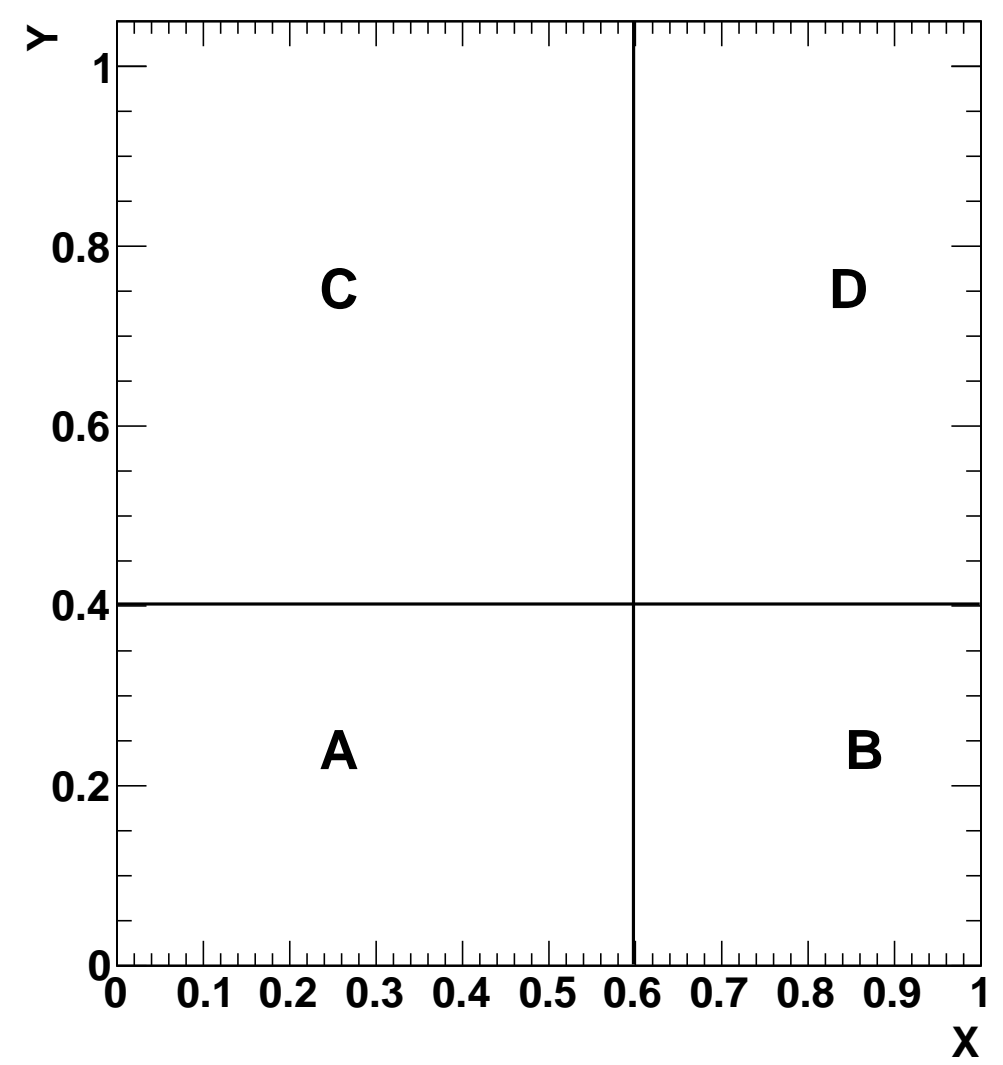

Figure E.1: The 2-dimensional plane in which the variables used for estimating background in data is defined. The plane is broken up into 4 sections; A, B, C, and D.

Recalling the expression given for the two-bin purity in Eq. 5.2, the following expressions arise:

$$
\begin{gathered}
f_{d a t}=\frac{A}{A+C} \\
f_{s i g}=c \\
f_{b k g}=\frac{B}{B+D}
\end{gathered}
$$

where $c=\frac{A}{A+C}$. Subsituting the above expressions into Eq 5.2 the expression becomes:

$$
S=\frac{A D-B D}{D c-B(1-c)}
$$

as $\mathrm{c} \rightarrow 1$ (assuming no signal in region $\mathrm{C}$ ) Eq. E.5 gives the same expression as 
Eq. E.3, thus showing that the two-bin purity is an equivalent method to determine the background estimation in data as the 'ABDC' method. 


\section{APPENDIX F}

\section{PULL DISTRIBUTIONS}

The following plots show the pull distributions for all $\left|\eta^{*}\right|$ bins. The mean and $\sigma$ from the Gaussian fitting is reported on the upper right side of the plot. The mean for all the pull distributions are at about 0 while the width of the distributions are less than 1, indicating that the purity uncertainty is overestimated. The uncertainties were adjusted (values shown in Table 5.3) using the following expression:

$$
\Delta P_{\text {corr }}=\sigma * \Delta P
$$

where $\sigma$ is the width reported by the Gaussian fit and $\Delta P$ is the original purity uncertainty.

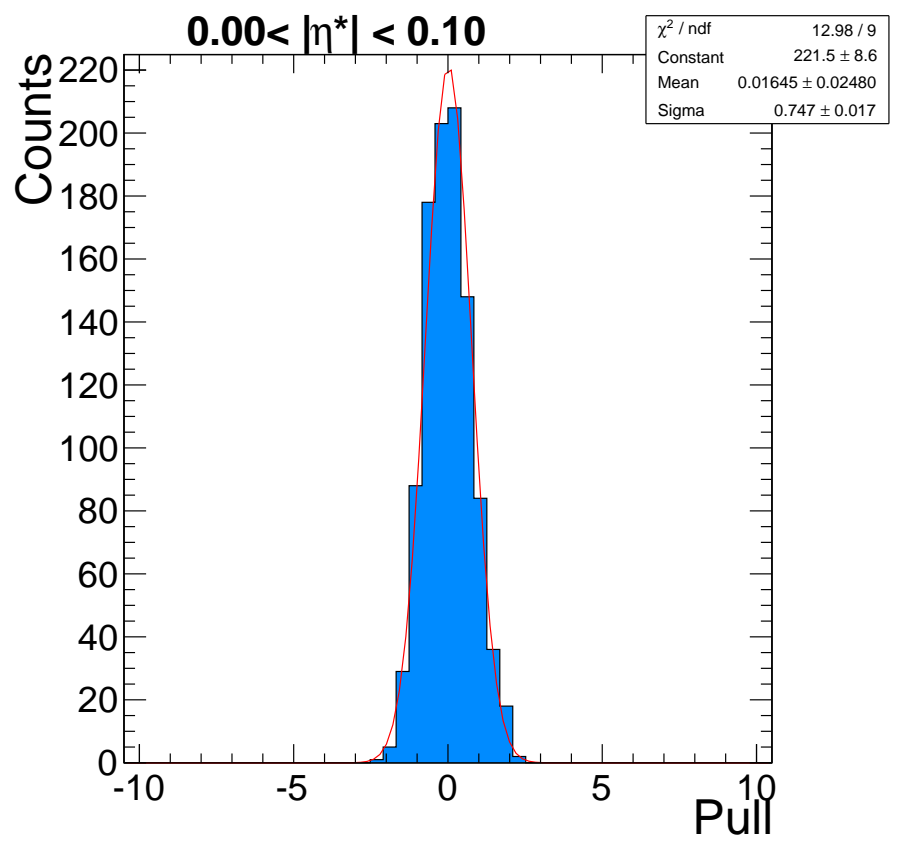

Figure F.1: Purity pull distribution for candidates in pseudo data with $0.0<\left|\eta^{*}\right|<0.1$. 


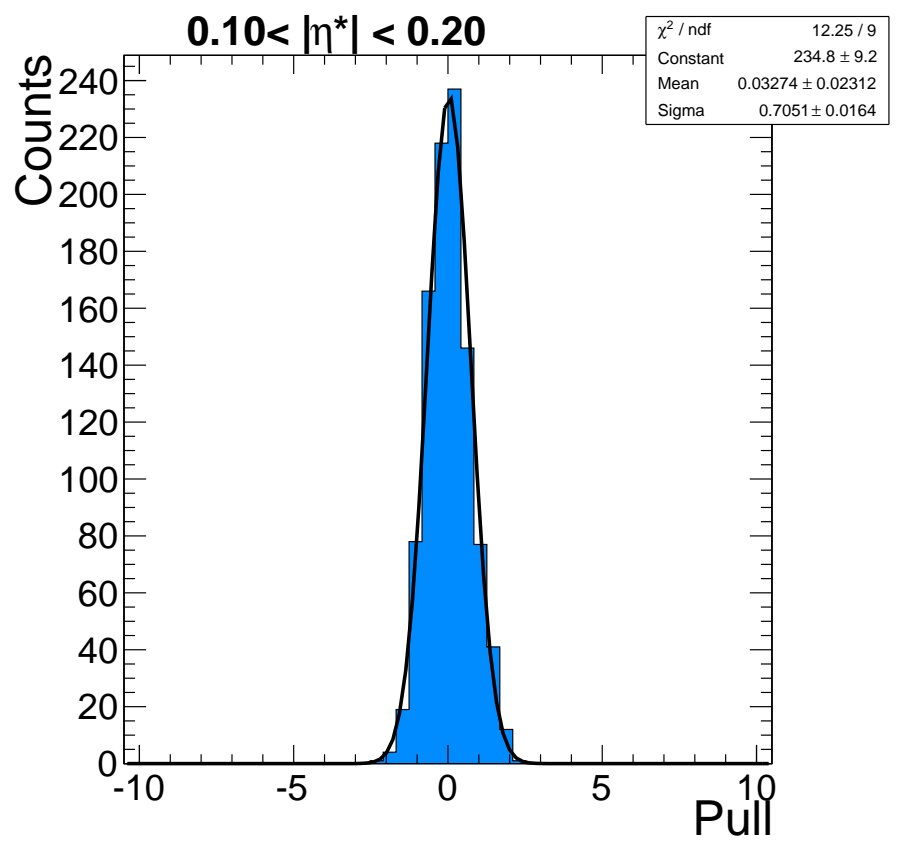

Figure F.2: Purity pull distribution for candidates in pseudo data with $0.1<\left|\eta^{*}\right|<0.2$.

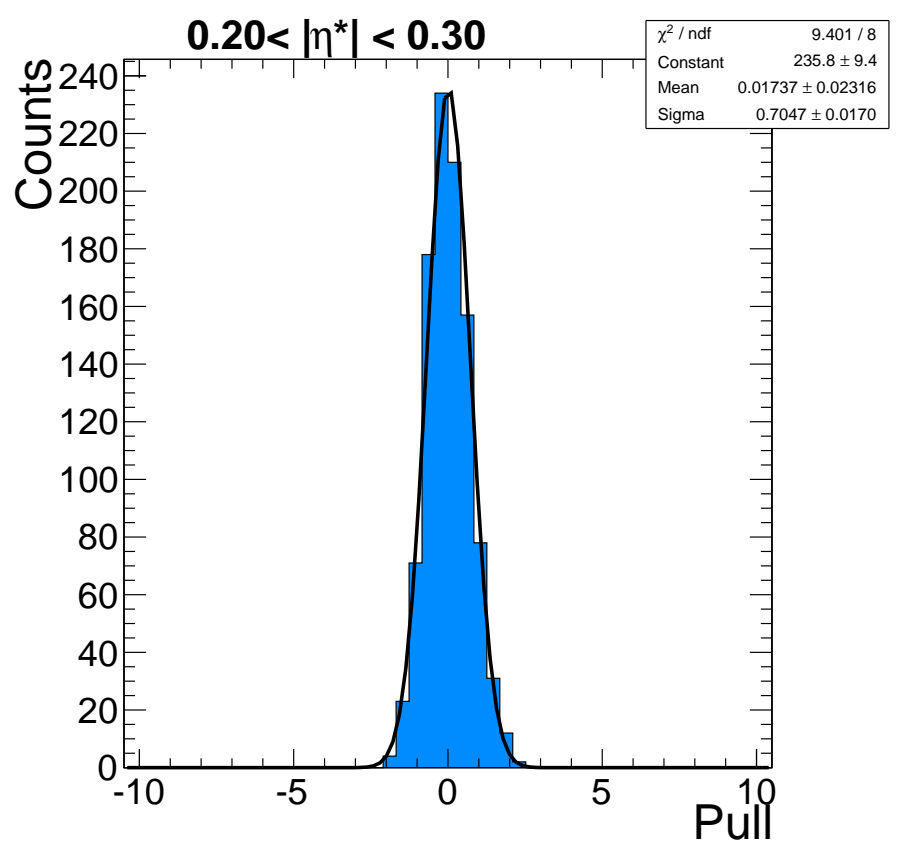

Figure F.3: Purity pull distribution for candidates in pseudo data with $0.2<\left|\eta^{*}\right|<0.3$. 


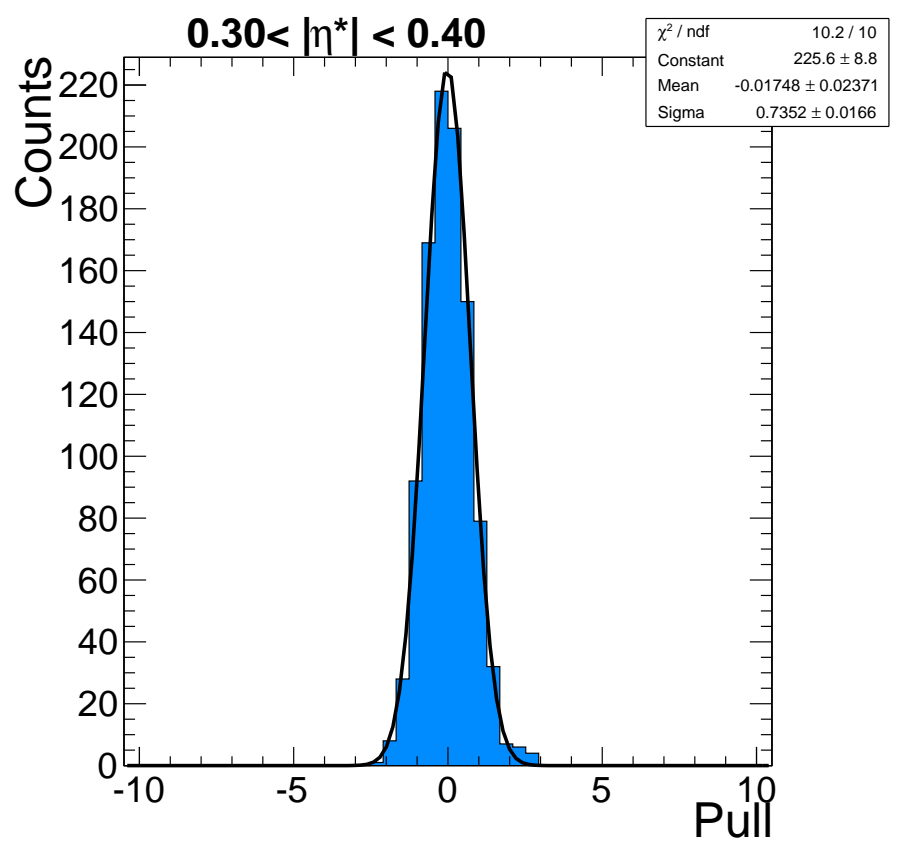

Figure F.4: Purity pull distribution for candidates in pseudo data with $0.3<\left|\eta^{*}\right|<0.4$.

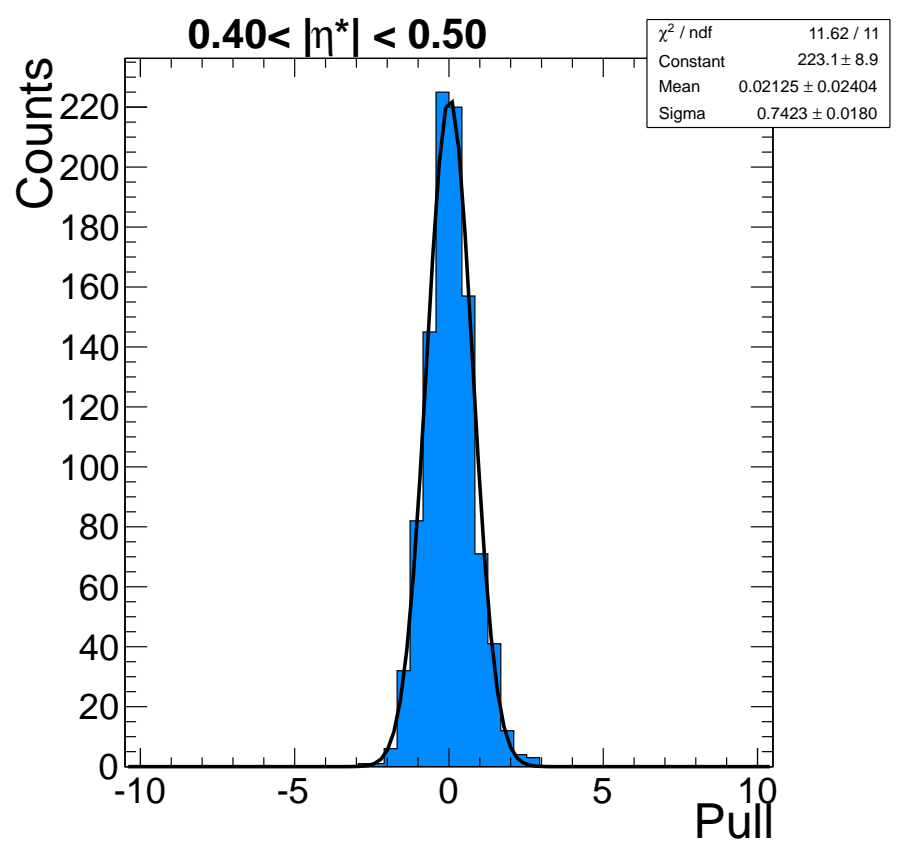

Figure F.5: Purity pull distribution for candidates in pseudo data with $0.4<\left|\eta^{*}\right|<0.5$. 


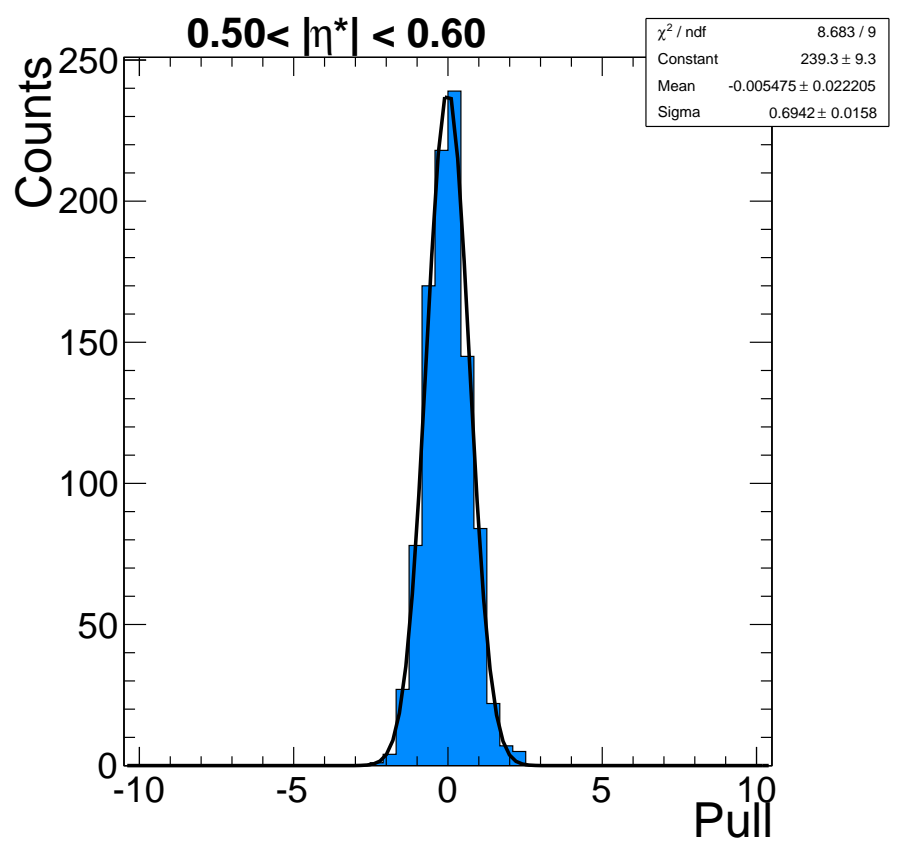

Figure F.6: Purity pull distribution for candidates in pseudo data with $0.5<\left|\eta^{*}\right|<0.6$.

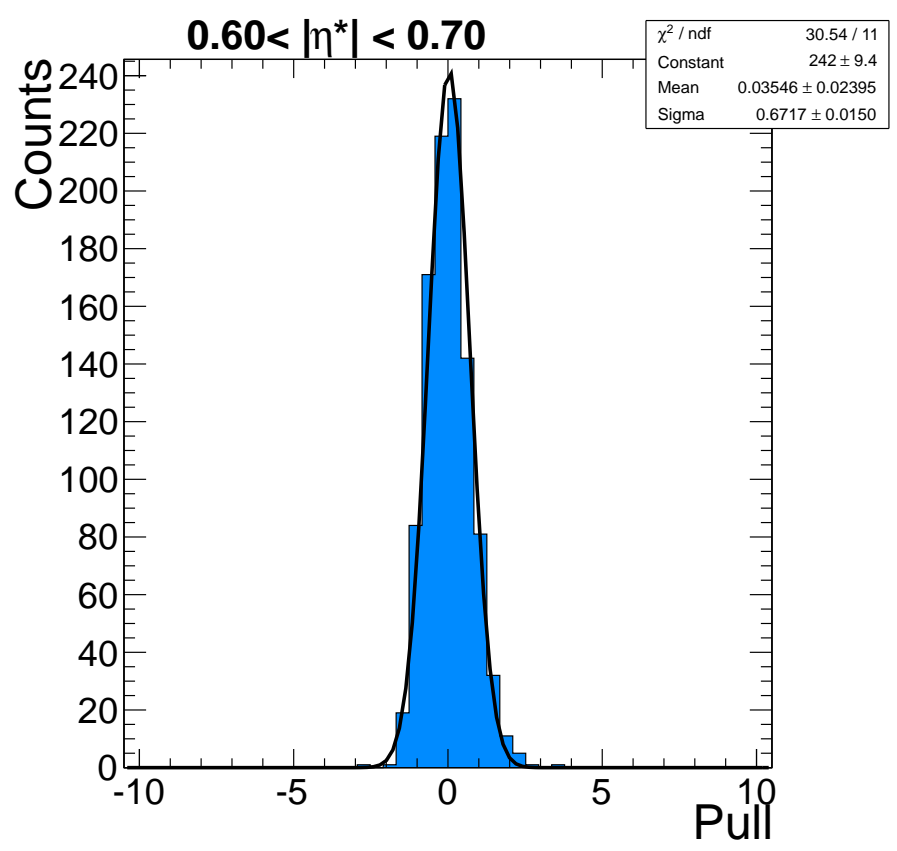

Figure F.7: Purity pull distribution for candidates in pseudo data with $0.6<\left|\eta^{*}\right|<0.7$. 


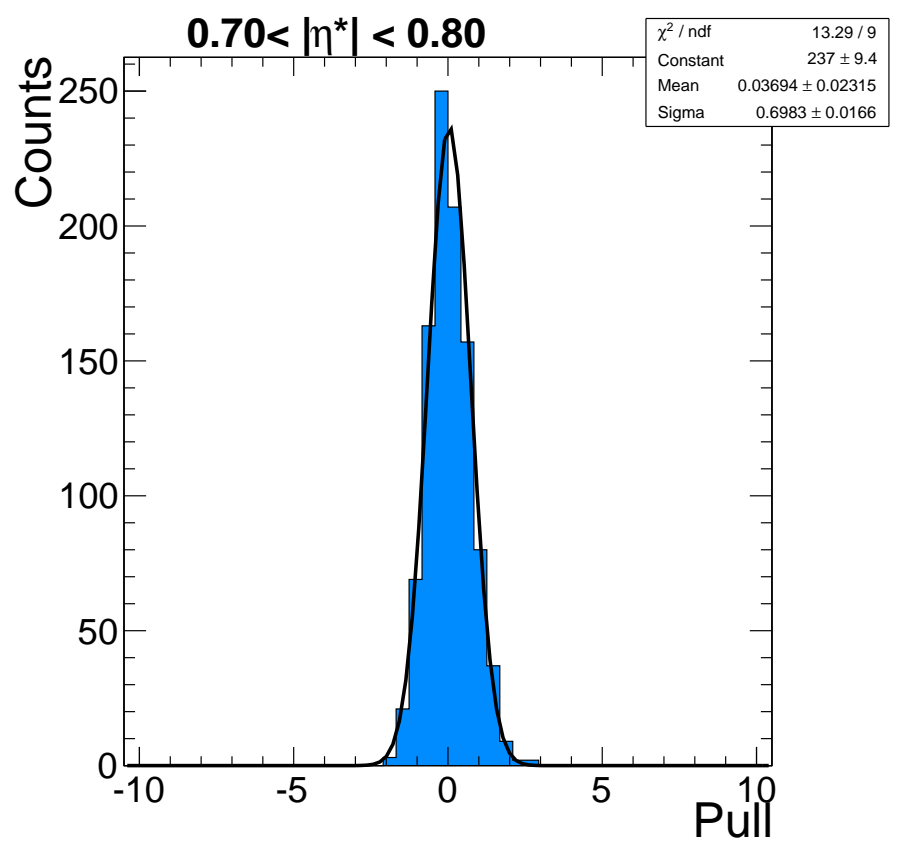

Figure F.8: Purity pull distribution for candidates in pseudo data with $0.7<\left|\eta^{*}\right|<0.8$.

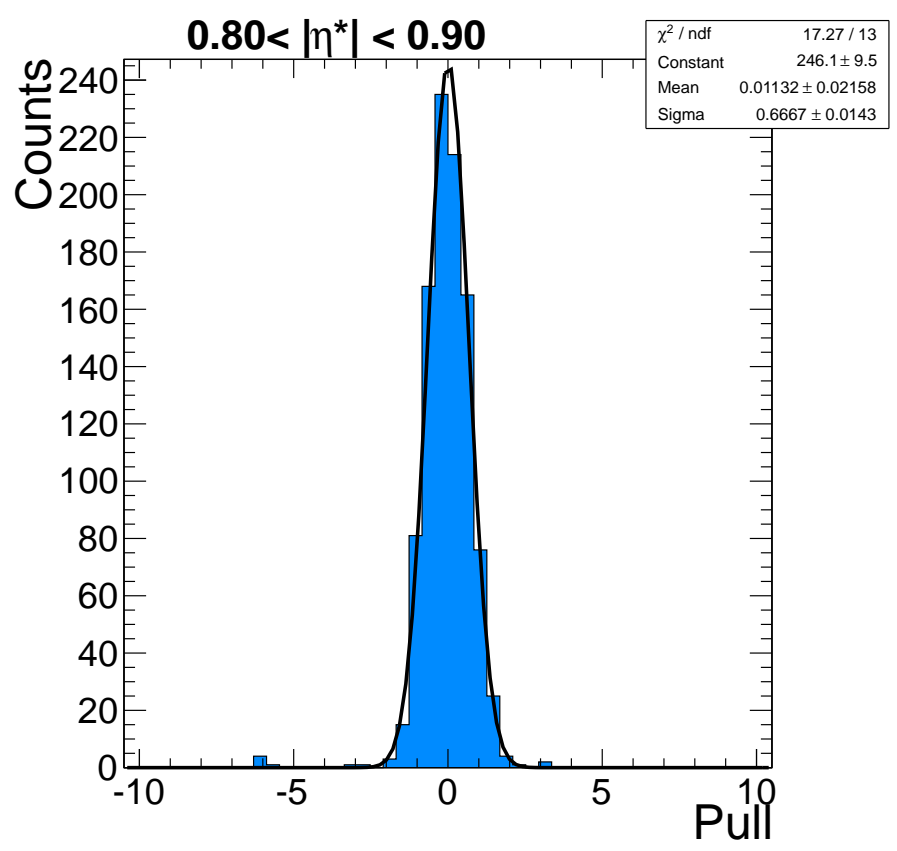

Figure F.9: Purity pull distribution for candidates in pseudo data with $0.8<\left|\eta^{*}\right|<0.9$. 


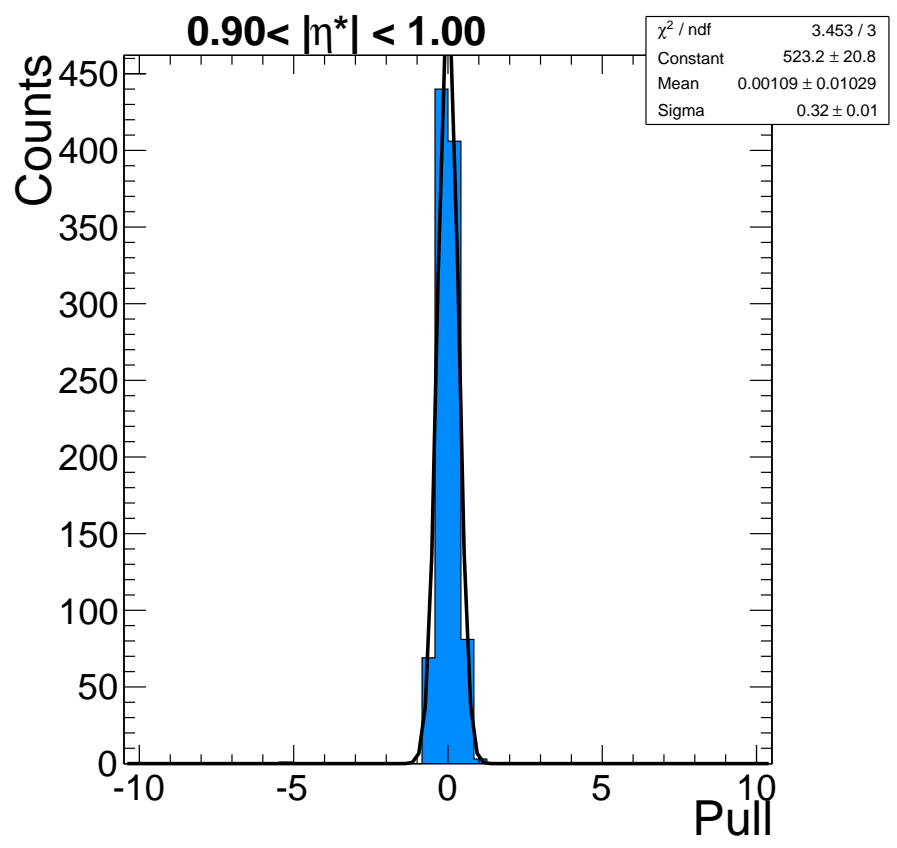

Figure F.10: Purity pull distribution for candidates in pseudo data with $0.9<\left|\eta^{*}\right|<1.0$.

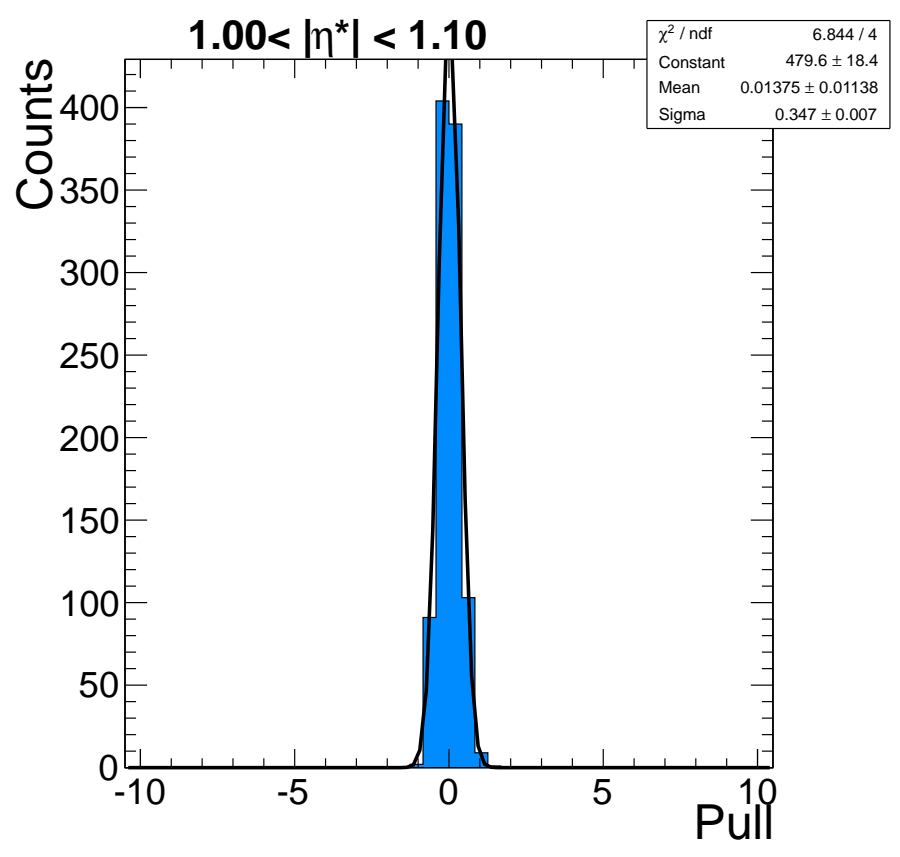

Figure F.11: Purity pull distribution for candidates in pseudo data with $1.0<\left|\eta^{*}\right|<1.1$. 


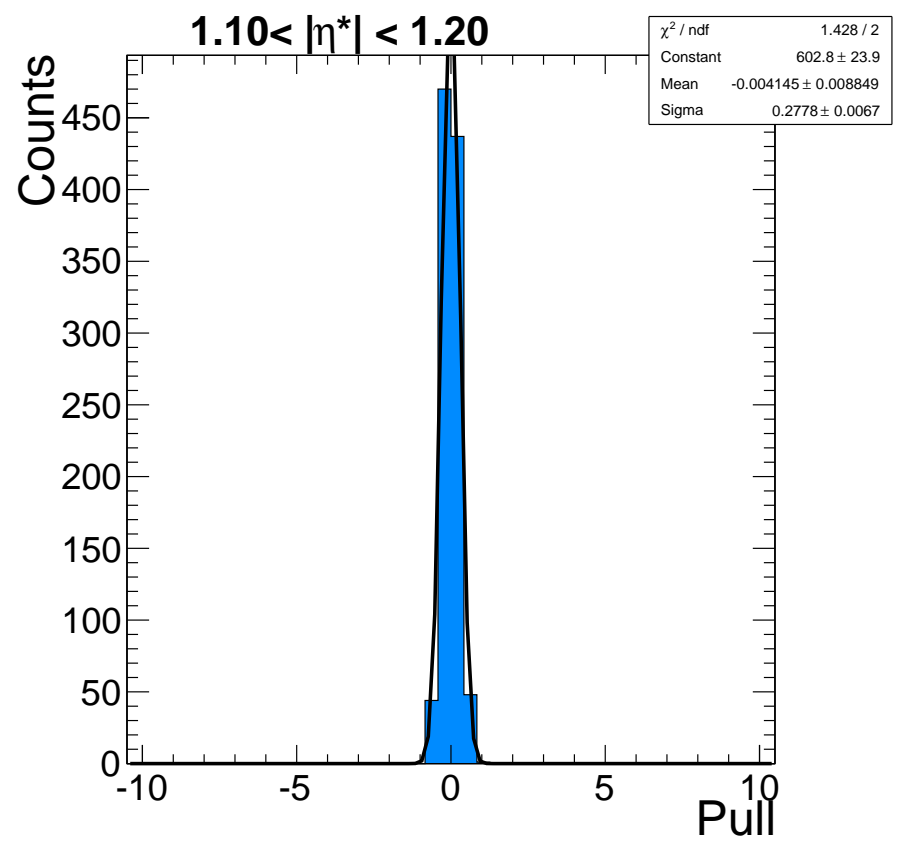

Figure F.12: Purity pull distribution for candidates in pseudo data with $1.1<\left|\eta^{*}\right|<1.2$.

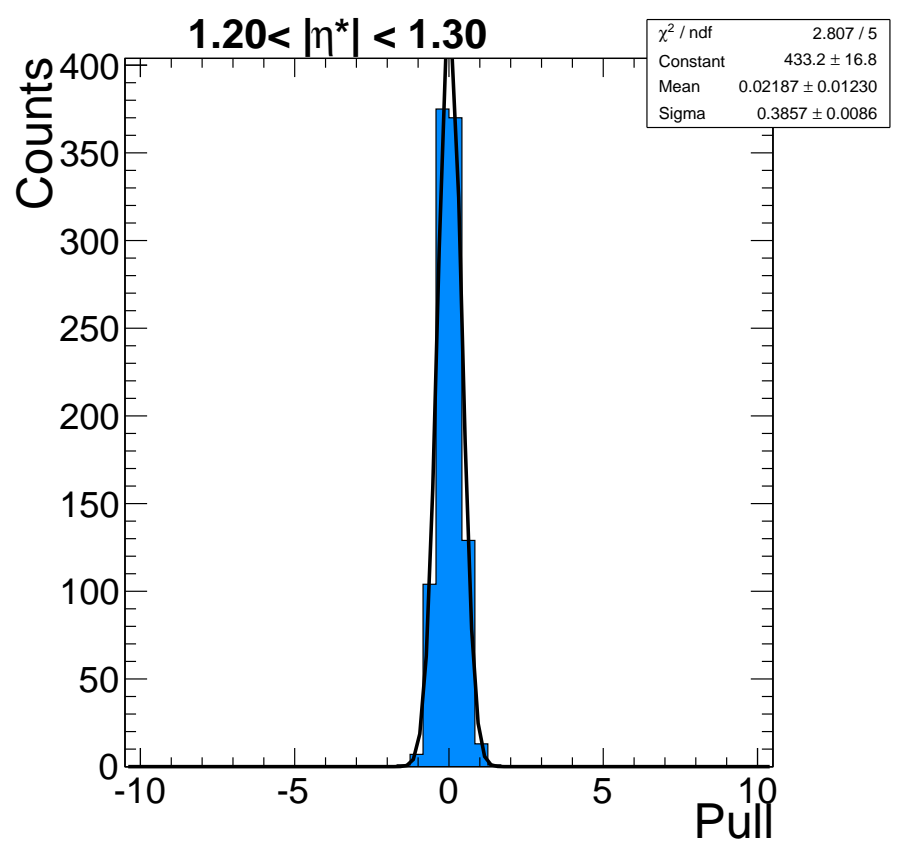

Figure F.13: Purity pull distribution for candidates in pseudo data with $1.2<\left|\eta^{*}\right|<1.3$. 


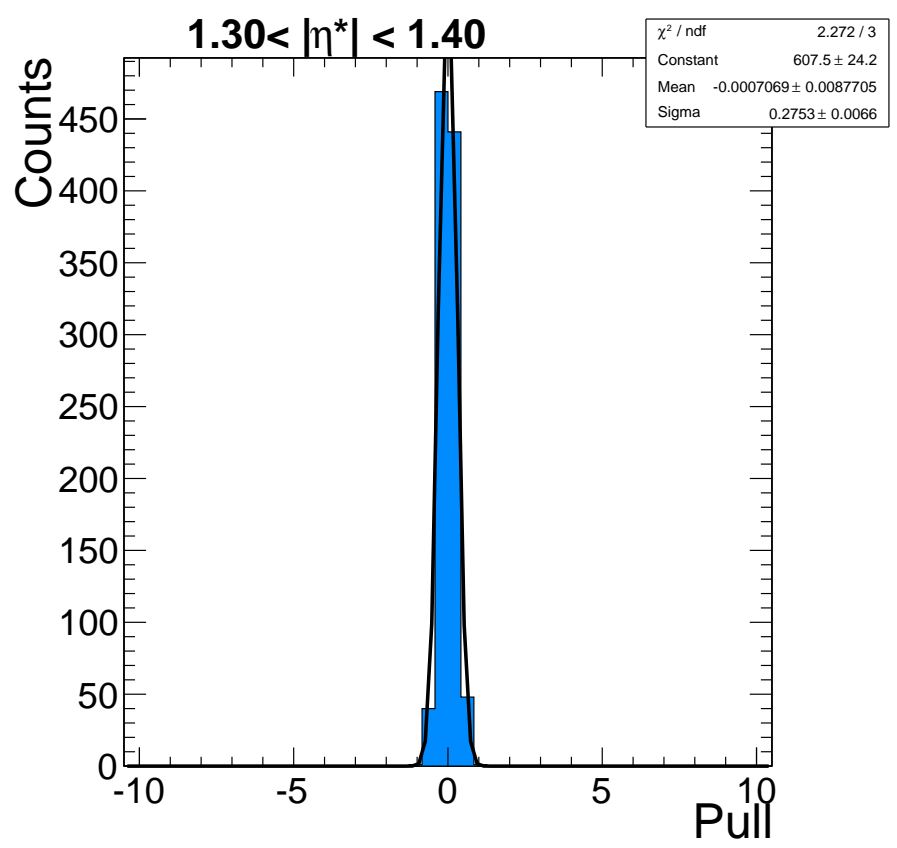

Figure F.14: Purity pull distribution for candidates in pseudo data with $1.3<\left|\eta^{*}\right|<1.4$. 


\section{APPENDIX G UNFOLDING THE ANGULAR DISTRIBUTION}

Measurements of physical observables can be distorted as particles traverse the detector. These distortions are caused by finite resolution or limited acceptance of the detector. An expression relating the distorted and the true distribution of physical observables is written as:

$$
\hat{R} t=m
$$

where $t$ is the true distribution, $m$ is the measured distribution and $\hat{R}$ is known as the response matrix. The response matrix describes the distortions expected of measured observables and is usually built through the use of simulated events of particles traversing the detector [67]. Eq. G.1 can be re-organized in terms of solving for the true distribution:

$$
t=\hat{R}^{-1} m
$$

where the response matrix is now inverted and operates on the measured observable $m$. Using Eq. G.2 we can now unfold what the true distribution is for measured observables. There are a variety of techniques used to unfold distributions in data, however we will only focus on the methods used for this analysis.

This analysis focused on unfolding the angular distribution in the CM frame of prompt photon candidates. The response matrix was built using Monte Carlo generated events with particles simulated going through the CMS detector. The generator level $\left|\eta^{*}\right|$ information fills the rows of the matrix while the reconstruction $\left|\eta^{*}\right|$ fills the columns. A 2-D representation of the response matrix is shown in Fig. G.1.

It is important to note the high occupancy of the diagonal terms in the matrix. Having the diagonal terms with non-zero values ensures the matrix can be easily inverted for the purpose of unfolding the measured angular distribution. 


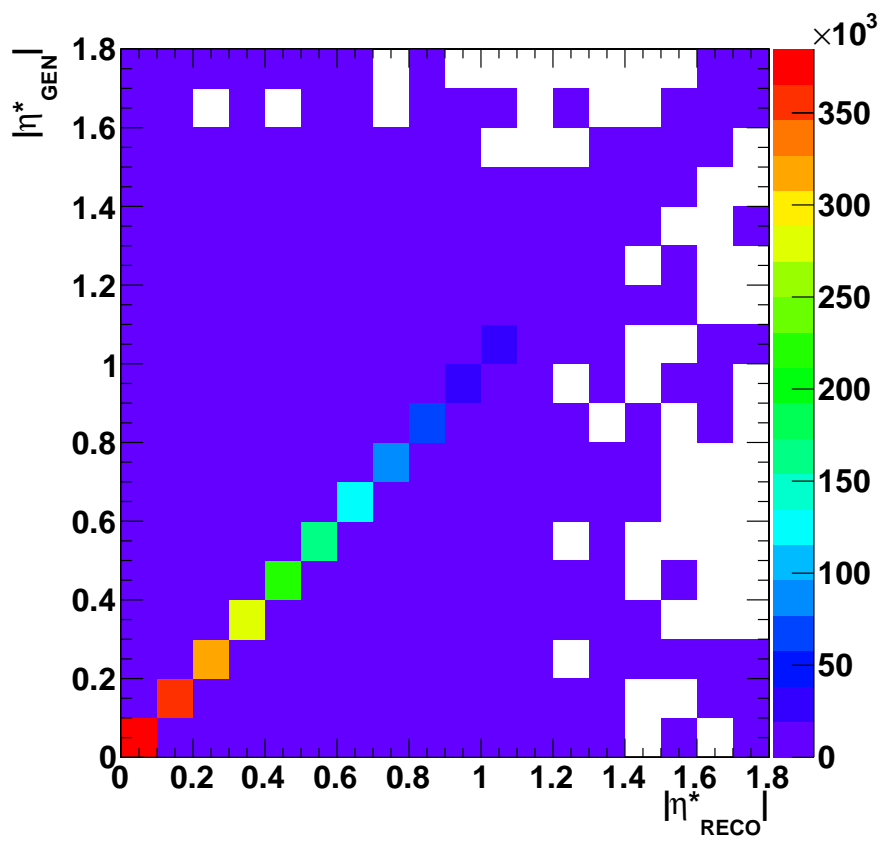

Figure G.1: The 2-D representation of the response matrix.

The RooUnfold package was used to unfold the angular distribution in this analysis [68, 69]. RooUnfold interfaces to the R00T data analysis package program and implements various unfolding techniques at the same time. The advantage of this package is that it implements various unfolding techniques commonly used in high energy physics to easily compare their results. The package also propagates the systematic uncertainty associated with unfolding measured distributions with little difficulty. The following unfolding techniques are implemented in RooUnfold and were used in this study:

- Bin-by-bin

- D’Augostini

- Singular Value Decomposition

- Matrix Inversion 


\section{Bin-by-bin}

The bin-by-bin unfolding technique is simply a correction factor. It measures the difference between the reconstructed and the generated distributions (from MC) for the variable to be unfolded and assigns it as the correction factor for the measured distribution.

\section{Matrix Inversion}

The entries in the 2-D response distribution shown in Fig. G.1 and the measured distribution are entered into matrices and entered in Eq. G.2. Since the 2-D distribution is not singluar (none of the diagonal terms are 0) it can easily be inverted. The distribution resulting from multiplying the inverted matrix with the measured matrix is the unfolded solution.

\section{D’Agostini}

D'Agostini unfolding as an iterative technique in which the reconstructed and generated distributions from MC are used to create a smearing matrix which gives the probability that the outcome of the measured distribution is caused by a physics process [70]. In the case of unfolding the angular distribution, the resolution effects of the electromagnetic calorimeter is the process which gives the resulting measured distribution. This matrix is then used to generate one distribution at a time, the idea being that each iteration agrees more with the generated distribution. This analysis used four iterations, the result is shown on the top left plot of Fig. G.2

\section{Singular Value Decomposition}

SVD unfolding may be used in the case when the inverted matrix of Eq. G.2 varies widely due to statistical fluctuations. These statistical fluctuations are 
smoothed out through regularization [67]. The regularization parameter in this analysis is given the same value as the number of bins in the measured distribution, as suggested by the literature.

Fig. G.2 shows the unfolded solutions using the techniques discussed. Not only is there little difference between the techniques, but there is also little difference between the unfolded and measured distribution. Therefore, no unfolding is made in the analysis and instead the difference between the unfolded and measured distribution is taken as a systematic uncertainty in the jet $\mathrm{p}_{T}$ resolution. 

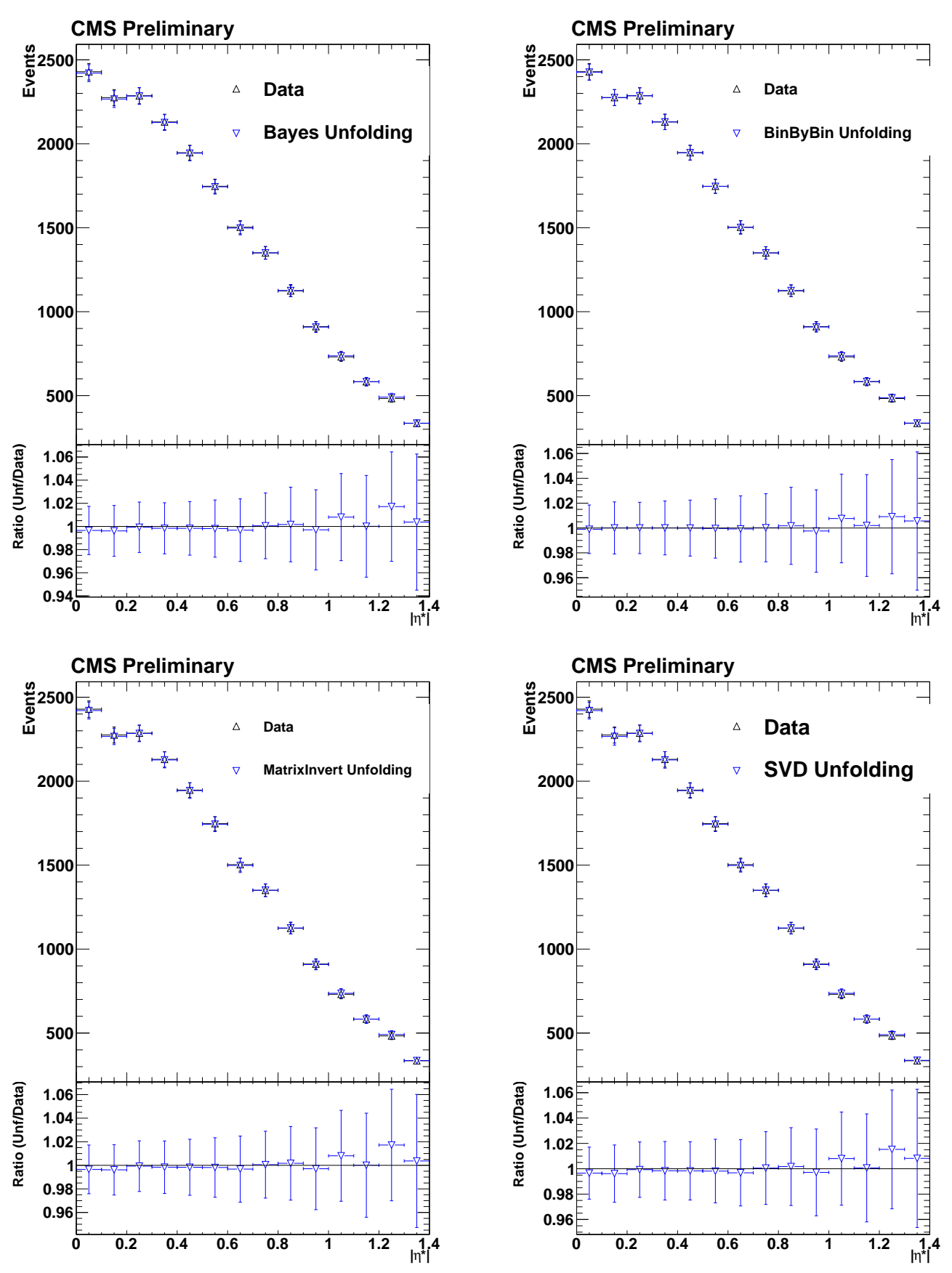

Figure G.2: Results for all four unfolding techniques, the upper left distribution was unfolded using the D'Augositini unfolding technique, the upper right distribution was unfolded using the Bin-by-Bin technique, the lower left distribution was unfolded using the Singular Value Decomposition (SVD) technique, and the lower right distribution was unfolded using the matrix inversion technique. All unfolded results vary by less than $1 \%$ from their measured distributions, as shown by the ratio plots located directly beneath each distribution. 


\section{APPENDIX $\mathrm{H}$}

\section{FRAGMENTATION PHOTONS}

As a side study, the contribution of fragmentation photons was studied by varying $\mathrm{Iso}_{E C A L}$ and $\mathrm{Iso}_{H C A L}$ by $\pm 1 \mathrm{GeV}$ each. The idea is that the looser isolation criteria will allow more fragmentation photons while the tighter criteria allows less. The presence of fragmenation photons would be evident at the tail-end of the $\left|\eta^{*}\right|$ distribution. An increase in more fragmentation photons would result in a less-downward slopping $\left|\eta^{*}\right|$ distribution while a decrease in fragmentation photons would result in a more-downard slopping distribution. Fig H.1 shows the $\left|\eta^{*}\right|$ distribution using the nominal set of isolation cuts, loose isolation cuts and tight isolation cuts. There appears to be no effect on the amount of fragmentation photons allowed in the sample. To ensure that these varied isolation distributions are correct, they are compared to NLO predictions, as shown in Fig. H.2 which show they are in good agreement.

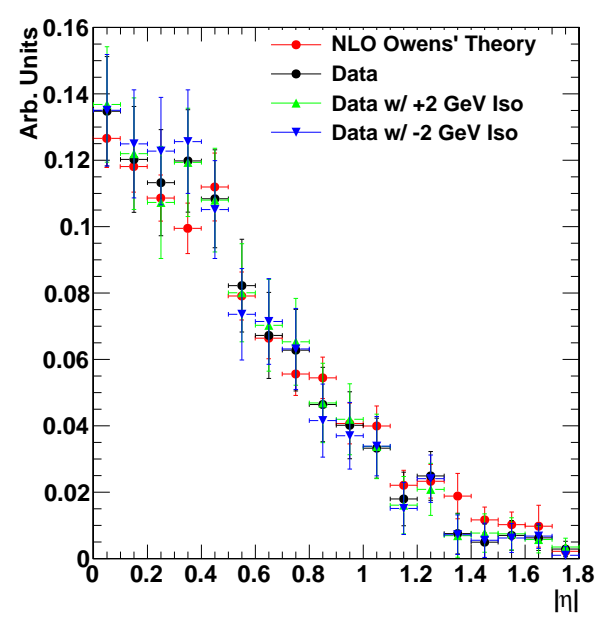

Figure H.1: Angular distribution for the nominal (black), loose (green) and tight (blue) isolation criteria overlaid with NLO theory predictions. 

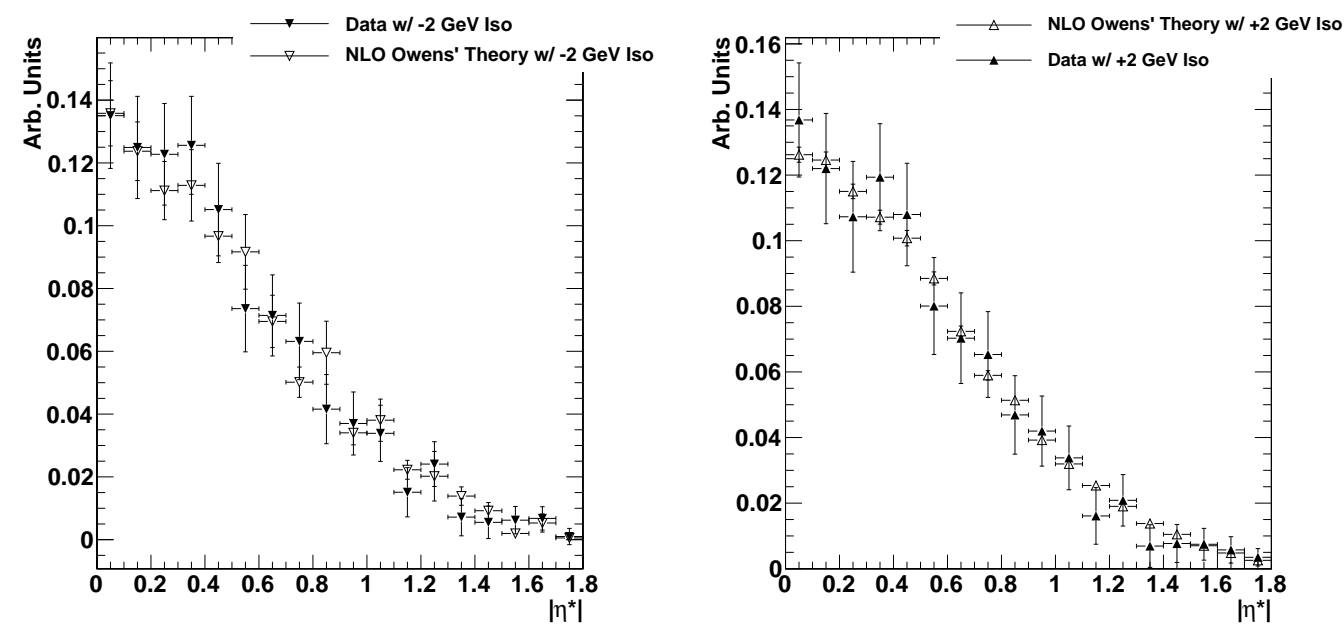

Figure H.2: Angular distribution when isolation is tight (left) and when isolation is loose (right). Both figures have their NLO theoretical prediction overlaid as well as the theory/data ratio (below) showing good agreement. 


\section{APPENDIX I}

\section{PILE-UP}

When making distributions with MC, it is important (especially when calculating the identification cut efficiencies) to re-weight with respect to the observed pile-up effect in data. As mentioned in the main body text, the pile-up was calculated using the number of primary interactions in each event. The number

of primary interactions was plotted in the MC sample used in this analysis and in data. The ratio between the those distributions in data and MC was used to re-weight $\mathrm{MC}$ in order to properly reflect the pile-up conditions observed in data. While variables such as the $p_{T}$ and isolations are affected by pile-up the template used in this analysis to estimate the purity, $\sigma_{i \eta i \eta}$ was not. This is shown in Figs. I.1, I.2 and I.3 where the $\sigma_{i \eta i \eta}$ distributions with different number of primary vertices, the mean value of those distributions as a function of number of primary vertices and the RMS of those distributions as a function of number of primary vertices are shown, respectively. 


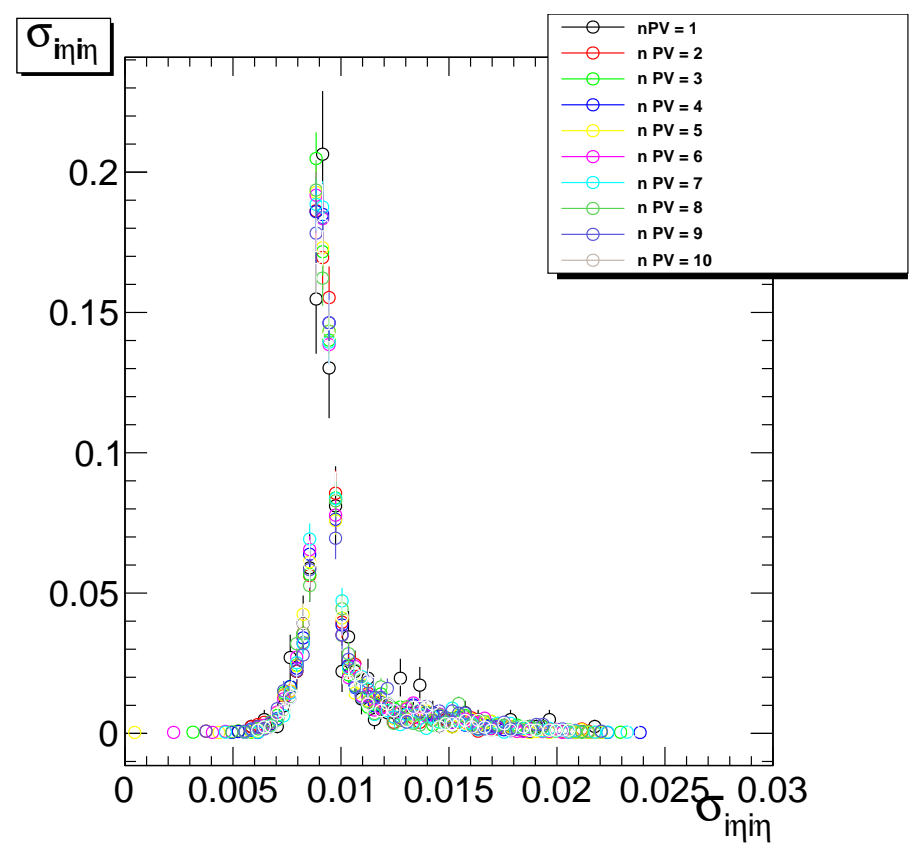

Figure I.1: The template distribution $\sigma_{i \eta i}$ for different number of primary vertices. The shape of the distribution shows no sign of change with different number of primary vertices.

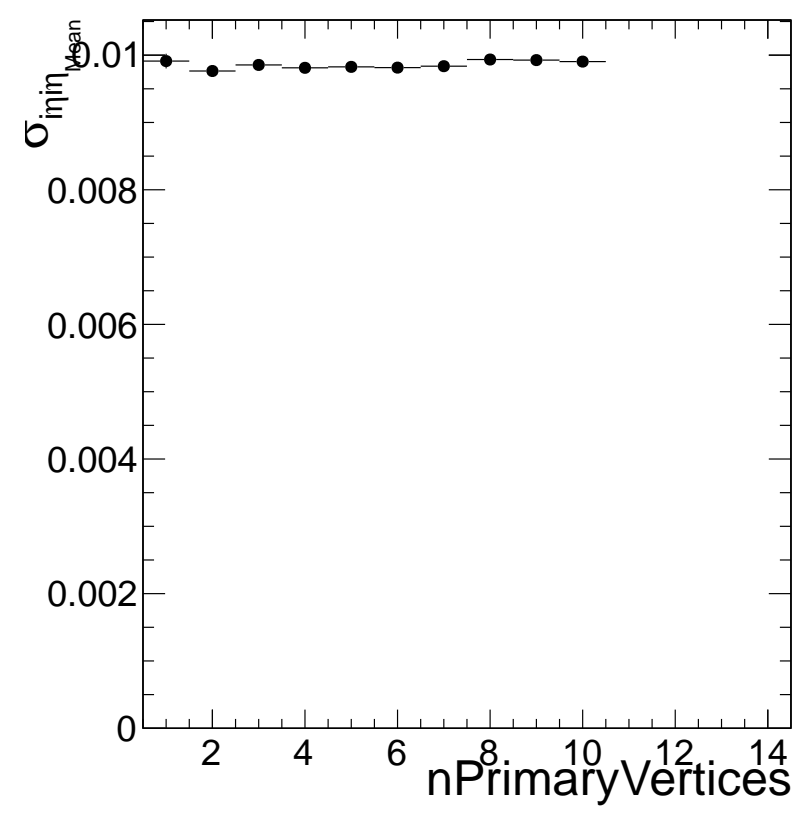

Figure I.2: The mean value of each $\sigma_{i \eta i}$ distribution as a function of number of primary vertices. The mean is constant. 


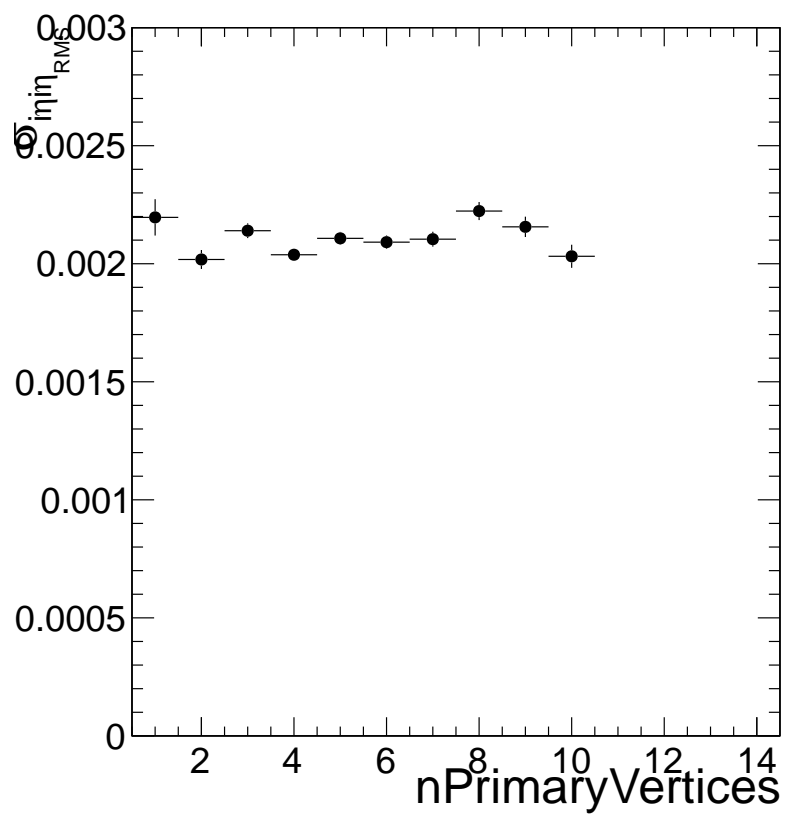

Figure I.3: The RMS of each $\sigma_{i \eta i \eta}$ distribution as a function of number of primary vertices. The RMS is constant. 


\section{APPENDIX J \\ CORRECTING $f_{b k g}$ - A SECOND APPROACH IN MEASURING}

$\left|\eta^{*}\right|$

A second method used to calculate the purity involved correcting the term $f_{b k g}$, which corrected the fraction of candidates with $\sigma_{i \eta i \eta}<0.01$ in the background template such that:

$$
f_{b k g}^{\prime}=f_{b k g}+\left(f_{b k g}^{q c d_{p h o I d}}-f_{b k g}^{q c d_{s b s}}\right)
$$

where $f_{b k g}^{q c d_{p h o I d}}$ represents the fraction of candidates in the background template with $\sigma_{i \eta i \eta}<0.01$ obtained from QCD MC with photon identification cuts applied and $f_{b k g}^{q c d_{s b s}}$ represents the same fraction obtained from QCD MC with the side band subtraction cut applied. The purity term in the angular distribution expression shown in Equ. 7.1 is affected by correcting $f_{b k g}$, which in term causes the angular distribution to vary. The motivation behind this method was to find a way to reduce the correlated uncertainty associated with the background shape. However, this method shows a larger fluctuation from the theoretical NLO prediction. Correcting $f_{b k g}$ obtained from the data-driven background template gives the distribution shown in Fig. J.1. This technique does not appear to assist in gaining further knowledge about the angular distribution or how to reduce the correlated uncertainties calculated for this analysis. 


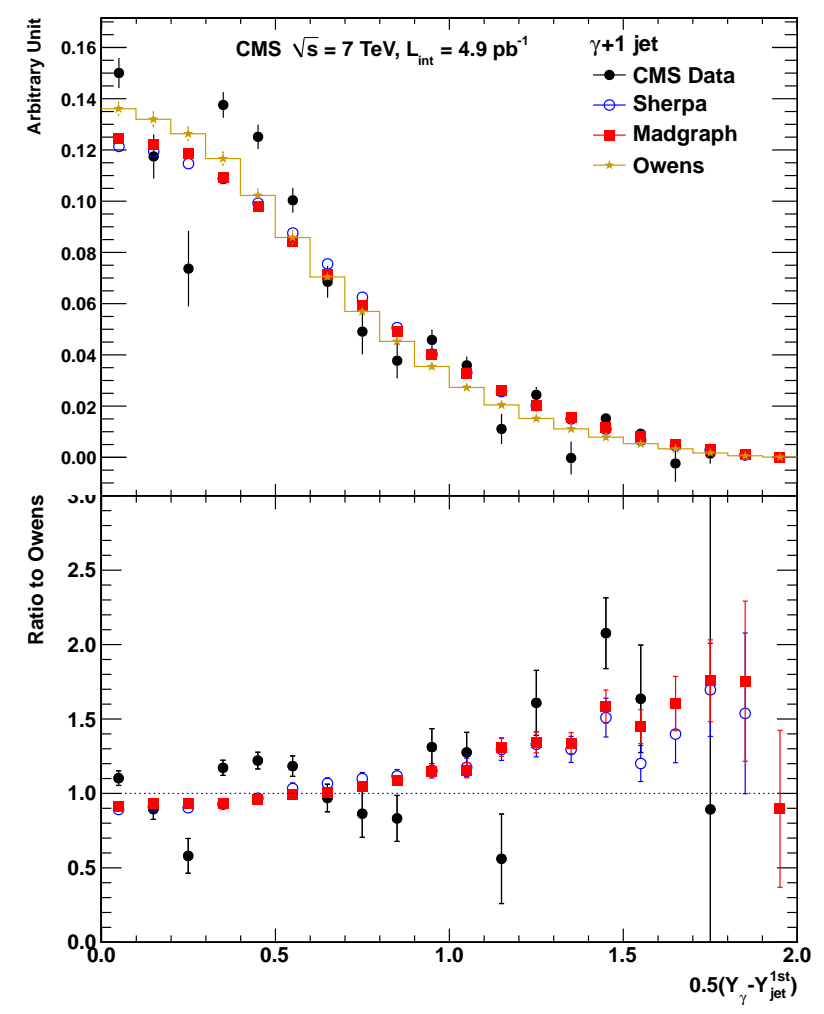

Figure J.1: The angular distribution, with $f_{b k g}$ corrected which affects the purity term in the angular distribution expression and hence the overall distribution. 
VITA

\section{VANESSA GAULTNEY WERNER}

March 4, 1984 Born, Miami, Florida

2006

B.S., Physics

Florida International University

Miami, Florida

PUBLICATIONS AND PRESENTATIONS

CMS Collaboration. "The CMS Experiment at the CERN LHC", Submitted to JINST (2008). doi:10.1088/1748-0221/3/08/S08004

CMS Collaboration. "Measurement of the Isolated Prompt Photon Production Cross Section in pp Collisions at sqrt(s) $=7 \mathrm{TeV}$ ", Submitted to Physical Review Letters (2010). 10.1103/PhysRevLett.106.082001

Angular Distribution of Photons in Gamma+Jet Events, Presented at the 78th Annual Meeting of the Southeastern Section of the American Physical Society, Roanoke, VA, October 2011

Unfolding the Photon $p_{T}$ Spectrum, Presented at the CMS Special QCD/FWD Unfolding Workshop at the European Organization for Nuclear Research, Geneva, Switzerland, September 2010

First Look at Photons for the CMS Experiment, First Prize Poster Presentation at the US CMS Collaboration Meeting at Brown University, Providence, RI, May 2010

Inclusive Photons at CMS, Presented at the American Physical Society April Meeting 2010, Washington, D.C. February 2010

Photon+Jet Studies, Presented at the USCMS JTerm IV, Batavia, IL, August 2009 